

UNITED STATES DEPARTMENT OF COMMERCE • Luther H. Hodges, Secretary NATIONAL BUREAU OF STANDARDS - A. V. Astin, Director

\title{
Mechanical Behavior of Crystalline Solids
}

\author{
Proceedings of a Symposium \\ April 28-29, 1962
}

Held under the auspices of the Ceramic Educational Council of the American Ceramic Society, with the cooperation of the National Bureau of Standards, and under the sponsorship of the Edward Orton Junior Ceramic Foundation and the Office of Naval Research. The Symposium took place at the 64th Annual Meeting of the American Ceramic Society in New York City.

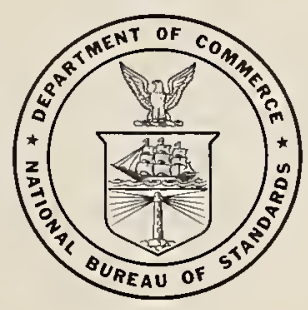

National Bureau of Standards Monograph 59

Issued March 25, 1963 
Nationa! Bureau of Standards

$$
\begin{aligned}
& \text { OCT 16 } 1963 \\
& 120,819 \\
& \text { QC100 } \\
& .4556 \\
& \text { cup.2 }
\end{aligned}
$$

Library of Congress Catalog Card Number: 63-60028 
1. Plastic Flow and Fracture of Crystalline Solids

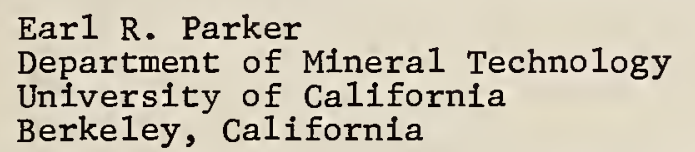

2. Theory of Dislocations: An Elementary Introduction

Roland de Wit

Metal Physics Section

Metallurgy Division

Nationa1 Bureau of Standards

Washington 25, D. C.

3. Observations of Dislocations

H. G. F. Wilsdorf

Research and Development Laboratories

The Franklin Institute

Philadelphia 3, Pennsylvania

4. Fracture Mechanisms in Crystalline Ceramics $y$

T. L. Johnston

Metallurgy Department

Scientific Laboratory

Ford Motor Company

Dearborn, Michigan

5. Strength of Ceramic Crystals

J. J. Gilman

Division of Engineering

Brown University

Providence, Rhode Island

6. A Review of the Effect of Microstructure on Mechanical Behavior of Polycrystalline Ceramics

W. D. Kingery and R. L. Coble

Division of Ceramics

Massachusetts Institute of Technology

Cambridge, Massachusetts 


\section{Introduction}

The physical properties of ceramic materials are in some ways very different from those of metals, and in other ways surprisingly similar. Ceramic materials tend to be strong but brittle, to be electrical insulators and rather poor thermal conductors, and to possess generally high melting points. Metals, on the other hand, are often ductile, possess appreciable electrical conduction, and have generally lower melting points than do ceramic materials. However, real similarities exist, and in particular it is found that the same general physical and chemical processes occur in both, alike in kind but differing in detail. Both are crystalline solids, and the physical properties of both are strongly sensitive to the presence of crystal imperfections, such as vacant lattice sites, interstitial atoms, and dislocations. These imperfections can be described in somewhat the same terms in both kinds of materials, and react in much the same ways to external influences, producing much the same effects.

Hence there has built up, in recent years, a science of the defect properties of solids. This science deals in a rather generalized way with imperfect crystalline solids, and its application to metals, or ceramics, or even, probably, to polymers, is more a matter of detail than of basic principle. In order to review this science with respect to mechanical behavior, and in particular for ceramic materials, the Symposium of which this book forms the Proceedings was held.

The Symposium was designed to present a review of dislocations and dislocation behavior, and how these are related to the mechanical behavior of ceramic materials. Prof. Parker, in the first chapter, reviews general ideas relating to strain hardening, recovery, creep, and fracture, and introduces the concept of dislocations as used to understand these phenomena. In Chapter 2, Dr. de Wit discusses dislocations as entities in themselves, with particular emphasis on their geometrical aspects. Dr. Wilsdorf, in the third chapter, describes methods of observing and studying dislocations, with examples drawn from metals and nonmetals.

These first three chapters form a background of introduction and general ideas about dislocations. The next two chapters are concerned specifically with properties of ceramic materials, with emphasis on single crystals. In Chapter Four, Dr. Johnston discusses the mechanism of fracture and the role played by dislocations in the initiation and propagation of fracture. Dr. Gilman, in Chapter Five, considers the problem of obtaining high strengths, a problem for which dislocation motion is important but not dominant. In the last chapter, Profs. Kingery and Coble leave the properties of single crystals and review the ways in which the microstructure of polycrystalline ceramics influence the mechanical properties.

Joseph A. Pask, Chairman University of California

Alan D. Franklin, Cochairman National Bureau of Standards

W. Wurth Kriegel North Carolina State College

Karl Schwartzwalder AC Spark Plug Division General Motors Corporation 


\author{
Earl R. Parker
}

\title{
Introduction
}

Why should ceramists be interested in dislocations? Are not ceramic products truly brittle solids, except perhaps at high temperatures where plastic or viscous flow can occur? And even the high-temperature flow need not be associated with dislocations, as is evidenced by the case of glass which flows by a viscous, rather than plastic, process. Why then, should the subject of dislocations be of more than casual interest to those concerned with the use of inorganic nonmetallic materials near ambient temperature? The answer is that the world of materials is changing. Leaders in this field can no longer be just "ceramists" or just "metallurgists"; they must become materials engineers or materials scientists. The dislocations that bestow the invaluable properties of toughness and ductility upon metals also exist in nonmetallic crystals. The differences between the dislocations in metals and nonmetals are small. The fundamental characteristics of dislocations evaluated by studies with lithium fluoride apply equaliy well to metals. As a matter of fact, metallurgists during the past decade have learned more about flow and fracture from studies of nonmetalic crystals than they have from metals.

Gradually the realization has developed that nonmetallic crystals are not necessarily brittle at normal temperatures. From the facts accumulated over a period of about forty years, it seemed reasonable to conclude that certain nonmetallic materials, particularly cubic ionic crystals, should exhibit plastic properties like those normally associated with metals. The work of Joffe $[1]^{1}$ in 1924 on sodium chloride and the later work of Nye [2] on silver chloride strongly supported this conclusion, as did the recent extensive classical studies of Gilman [e.g., 3, 4, 5] on lithium fluoride. Exploratory investigations $[6,7]$ revealed that many of the cubic ionic crystals, including magnesium oxide, were ductile at room temperature when they were carefully prepared. Tests in compression, bending, and tension yielded elongations well in excess of ten percent. The problem of brittleness evolved into one of crack nucleation, because if cracking was suppressed, flow was found to be extensive. The studies of fracture that followed the earlier work on flow have shed a great deal of light on the mysteries that enshroud fracture processes. This subject will be reviewed in detail in a later chapter by $T$. L. Johnston.

There are at least two worthwhile objectives to this kind of research. One is purely scientific--a quest for knowledge about the physical phenomena involved in flow and fracture processes; the second is technological in nature and is concerned with exploring the possibility of producing useful ductile polycrystalline nonmetallic materials that can be processed by methods such as rolling, forging, extruding, machining, and welding, that are now applicable only to metals. Although advancement toward the latter goal has been slow and many people have become discouraged because of the lack of spectacular and rapid progress, the picture is far from hopeless. Little by little, as more is learned, the end point comes nearer. Optimism, however, should be tempered with judgment. No one versed in the field expects ionic crystals to exhibit the extensive ductility characteristic, for example, of copper and aluminum. However, it is well within the realm of possibility that polycrystaline magnesium oxide can be produced with ductility comparable with that of tungsten, once thought to be a hopelessly brittle metal, or, for that matter, with ductility similar to that of ordinary structural steel at low temperatures. In any event, the time has come when ceramists must be familiar with dislocation concepts of flow and fracture if they are to compete in the rapidly advancing field of materials.

\section{Dislocations}

The story of dislocations goes back about thirty years. Physicists [8] studying the mechanical behavior of crystals had concluded by 1926 that the yield strength should be about one-sixth of the shear modulus. Refinements [9] in the calculations indicated that the theoretical strength might be as low as one-thirtieth of the shear modulus, but even this figure was several orders of magnitude higher than the measured strengths. It seemed reasonable to assume, therefore, that the weakness of real crystals was due to some sort of structural defect--a conclusion supported by X-ray diffraction evidence which clearly showed that crystal lattices are not perfect. The problem thus resolved itself into answering the question: What sort of crystalline

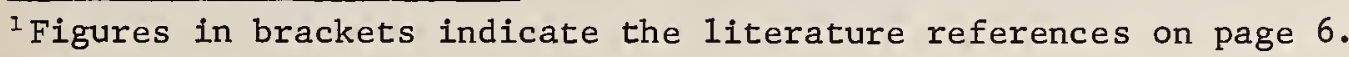


defect would have the characteristics necessary to account for the low value of yield strength and the phenomenon of strain hardening? The answer was provided in 1934 by Taylor $[10]$, Orowan [11, and Polanyi [12], who independently concluded that the defect conceived by Prandt1 [13] and Dehlinger [14] about 1928 had the required properties. This imperfection has since become known as the edge or line dislocation. An elementary form of the dislocation is shown in figure 1 . Here it consists of an imperfect square grid, the top half containing one more vertical row of atoms than the bottom half. In a three-dimensional model there would be a large number of identical planes evenly spaced in front of and behind the one shown; thus the defect would consist of a missing sheet of atoms in the lower half of the crystal model. A straight line through the lattice along the top of the missing sheet would coincide with the region of maximum lattice disturbance. It is for this reason that the defect is called a line or edge dislocation. (A dislocation of the "opposite sign" would exist if the missing sheet were above instead of below the slip plane.) Above and below the dislocation line, the lattice spacing gradually returns to the normal value. In the center of the disturbed region, the lattice is extremely distorted, atoms being squeezed together above the line and spread apart below it. This defect has the unique characteristic of being able to move through the lattice on a plane perpendicular to the plane of the missing sheet of atoms and in a direction perpendicular to the line of the dislocation. Very low stresses are required to cause dislocations to glide through a crystal lattice, and when such defects emerge on a free surface a "slip" offset one atomic spacing wide is produced. When many dislocations operate on the same plane, the slip offset becomes large enough to see with a light microscope, or even by eye. These large offsets are called "slip lines," and "slip bands" are groups of closely spaced slip lines.

The edge dislocation alone is not enough to account for plastic flow, because gross shearing of a slip plane must proceed gradually across the entire area, not only in the direction perpendicular to the edge dislocation but at right angles to this direction as wel1. It was for this reason that Burgers [15] in 1939 conceived of the screw dislocation. Unlike the edge dislocation, which extends perpendicular to the direction of slip, the screw dislocation is a lattice defect extending parallel to the slip direction. This second type of dislocation permits a wave of plastic flow to travel through the lattice in a direction at right angles to the direction of the slip movement. Figure 2 illustrates how this can happen. In this figure, the vector, $\mathrm{b}$, (called the Burgers vector) represents the magnitude and direction of slip.

Conceding the existence of dislocations, a necessary step in the early development of the theory, the next question to be answered was: Where do dislocations come from? Calculations clearly showed that there could not possibly be enough dislocations preexisting in metal crystals to account for the observed extensive plastic flow. Furthermore, X-ray examination showed without a doubt that the number of defects in crystals actually increased, rather than decreased, as plastic flow progressed. A11 available evidence indicated that dislocations actually multiply during deformation. How could this be? Again, the ingenuity of the theorist provided the answer. Simultaneously, F. C. Frank and W. T. Read [16] conceived of the now familiar Frank-Read source, a dislocation generator capable of creating an unlimited number of moving or "slip" dislocations. The Frank-Read concept is based upon the assumption (since verified) that dislocations that form in crystals during growth do not lie entirely on a single slip plane. In fact, some portions of the grown-in dislocations were presumed to have portions Iying in planes where slip was virtually impossible. Thus, only the part lying in the slip plane was free to move under an applied stress, but this part, being constrained at the ends where the line left the slip plane, could only bow as shown in figure 3. To understand this action, it is necessary to appreciate that, while dislocations can move through a lattice on any plane, the shear stress necessary to cause movement varies by several orders of magnitude for different planes. The easiest direction of movement is always in the direction of closest like neighbors, and the planes of easiest slip are those that have the highest density of atoms. It is natural, therefore, that slip will almost invariably occur only on those planes requiring the minimum stress for dislocation movement. These are the characteristic "slip planes" observed to operate when crystals are deformed. Rarely does the stress rise high enough to move dislocations on the "nonslip" planes.

Returning to figure 3 , the loop of the bowing dislocation line grows with increasing stress until it begins to swing around the pinned end points. This process continues until the line, growing and moving together behind the pinning points, eventually becomes a complete circle. At this stage, it breaks away from the source and becomes a freely moving dislocation "loop" that can continue outward to produce slip offsets on external surfaces. In the meantime, the process of the joining of the dislocation line to form a loop also recreates the source, as indicated in the sketches in figure 3. Thus the source can begin again to form a second loop, to be 
followed thereafter by an indefinite number of others. Conclusive evidence for the existence of Frank-Read sources is now available and, in addition, other kinds of sources have been discovered [17].

As inevitably happens, the solution of the source problem raised other questions. For example, why did a source stop functioning after a few hundred dislocations had been generated? It was known from electron microscope examinations of surfaces of deformed crystals that slip offsets might be hundreds, or even a thousand, of atomic distances, but that slip always stopped after relatively limited amounts of flow on any given slip plane. This is a manifestation of the phenomenon of strain hardening, which even today is not clearly understood.

\section{Strain Hardening}

The questions of what is strain hardening, and how and why does it occur have been the subject of speculation and analysis ever since its existence was recognized. G. I. Taylor [10] was the first to treat this problem quantitatively, and it has been the subject of repeated probes since his classical analysis in 1934. Examples of recent attempts to explain strain hardening are those of Basinski [18] ard Seeger et al [19]. It is interesting to note that after nearly thirty years of theoretical development, there is no general agreement among experts as to the details of what goes on inside crystals to cause them to become stronger when they are deformed. However, no one questions the seemingly self-evident fact that strain hardening is due to the interaction of the stress fields that surround dislocations. Stresses in the immediate vicinity of dislocations may reach values of a million pounds per square inch, and even though these stresses decrease inversely with the distance from the dislocation core, they may still be thousands of pounds per square inch many atomic distances away. This subject will be more fully discussed by $R$. deWit in the next chapter, so it will not be pursued here. The important point to remember is that strain hardening is caused by interactions of the stress fields that encircle dislocations.

The problem of strain hardening is complicated by the fact that this process involves the action of large groups of dislocations. Group behavior is complex because of the large number of ways in which dislocations can arrange themselves. Many kinds of interactions are possible, and it has been difficult to decide which interactions are important and which are trivia1. Part of the confusion has arisen because of erroneous or misleading interpretation of experimental results; the trouble has been largely due to the fact that it has not been possible, until recently when transmission electron microscopy was developed, to see individual dislocations in action. Consequently, theories of strain hardening had to be based on an assumed arrangement of dislocations in strained crystals. One of the most logical assumptions was that a series of dislocations emanating from a single source would pile up on the slip plane if the first dislocation encountered a barrier, such as a grain boundary or precipitated particle, through which it could not penetrate. This effect is shown schematically in figure 4, and figure 5 is a transmission electron micrograph showing an actual pileup of this type. Thus, both theory and experiment show that pileups are important. The analysis of this situation was first made by Burgers and Burgers [20] in 1938 who showed that a pileup would produce a "back stress" which would oppose the applied stress. Thus the stress acting on the source and causing the generation of dislocations would tend to become neutralized and the source would eventually cease to function. This concept has been extended further by others and has been pretty well substantiated experimentally (for more details, see reference [21]). This theory accounts well for the behavior of certain crystals, such as the hexagonal metals, that normally slip on only one plane, but does not satisfactorily explain the strain hardening behavior of cubic crystals which can slip simultaneously on several planes.

The cubic crystals, like the hexagonal metals, exhibit a low rate of strain hardening when slip occurs on a single plane. But, as figure 6 shows, when multiple slip occurs, the rate of hardening is much higher. What is the reason for this? The answer obviously must be associated with the interaction of dislocations generated and moving on intersecting slip planes. Such dislocations can react in several ways; one of the important reactions was proposed by Lomer [22] in 1951. He showed that two straight dislocations having Burgers vectors $a / 2[10 \bar{I}]$ and $a / 2[011]$ can unite to form a new dislocation having the vector a/2[110]. This new disIocation is of the edge type and could only glide on the (OOI) plane--a nonslip plane. The Lomer dislocation thus cannot move through the lattice and therefore can act as a barrier to slip dislocations moving toward it on the two intersecting slip planes. In this manner, new barriers can be created by the action of slip dislocations and it is barriers of this general type that are responsible for the higher rate of strain hardening exhibited by cubic crystals. 
Strain hardening is more complex than just described, but it is clear that it is due to the interactions of the stress fields that surround dislocations. In crystals where slip on several planes is possible, dislocations interact and become tangled in a complex manner, as illustrated by the transmission electron micrograph in figure 7 . Slip dislocations find it more and more difficult to penetrate these tangled barriers, and higher and higher stresses must be applied to cause flow to continue as the tangles develop.

\section{Recovery}

When a ductile crystalline material is deformed at room temperature and then reheated to successively higher temperatures, several changes occur to relieve the internal stresses generated by the presence of the strain-induced dislocation networks. Dislocation loops tend to become smaller and some may even disappear. This process happens rather rapidly and when it occurs the hardness drops, the internal stresses diminish, and, in metals, the electrical resistivity is lowered.

A second recovery process is the combination and annihilation of dislocations of opposite sign. This event generally requires the movement of dislocations in the direction perpendicular to the slip plane by a process known as "climb." Dislocation climb is necessary before unlike dislocations can unite because they are generally not located on the same slip plane. In order for dislocations such as the one shown schematically in figure 1 to climb out of the active slip plane, lattice vacancies must deposit al1 along the dislocation line. When this occurs, the line will move one plane higher in the lattice. A continuation of this process will cause the dislocation to migrate onto other parallel slip planes far removed from the one on which it was generated. It is also possible, by the addition of extra atoms, for a dislocation to migrate downward through the lattice. The climb process is relatively slow and its temperature dependence is exponential because its rate is governed by self-diffusion.

A third phenomenon contributing to recovery is the rearrangement of like dislocations into a boundary of the kind shown schematically in figure 8 . As predicted by theory, the arrangement shown in figure $8(\mathrm{~b})$ is a low energy metastable configuration. Figure' 9 is a transmission electron micrograph showing boundaries of this kind in an actual crystal. Boundaries of this kind form only where there is an excess of dislocations of one sign. Dislocation boundaries of this sort are called subboundaries, and the portions of relatively dislocation free crystal surrounded by such networks are called subgrains. The entire structure is called a "substructure."

Simple boundaries of the kind sketched in figure $8(\mathrm{~b})$ are not barriers to moving dislocations, but the more complex types normally present in deformed and heated crystals are good barriers. Subboundaries are very stable. They often remain in deformed metals even after heating to temperatures far above the normal recrystallization temperature. For example, in zinc single crystals such boundaries are stable at temperatures approaching the melting point; in nickel, subboundaries remain in polycrystalline material even after heating to temperatures in excess of $800^{\circ} \mathrm{C}$. Because subboundaries are relatively stable and because they resist penetration by moving dislocations, it is possible to increase the strength of an annealed commercial material by introducing a substructure in the grains. An example of the change in strength that can be thus produced is shown in figure 10. Polycrystalline nickel [23] was prestrained various amounts and then reheated one hour at $800{ }^{\circ} \mathrm{C}$. The amount of substructure increased with increasing amounts of prestrain, and the yield strength increased proportionally. Similar results were obtained with other metals. The presence of a substructure also has a profound effect upon the creep behavior of a material, as will be shown in the next section.

\section{Creep}

Many theories of creep have been formulated to explain the mechanical behavior of materials at elevated temperatures. Although these will not be discussed in detail, the important basic concepts will be considered. Dislocation climb seems clearly to be the rate controlling process. The activation energy for high-temperature creep [24] has been found experimentally to be the same as that for self-diffusion, the basic process regulating climb. Climb gives dislocations the degree of freedom required for them to detour around the barriers that had stopped their movements on their slip planes. As stuck dislocations leave their slip planes, the back stress at the dislocation source is reduced so that additional dislocations can be generated. Continuous operation of this process is responsible for creep. 
At creep temperatures, recovery can occur spontaneously. Experiments have shown that a substructure develops as creep progresses. The formation of a substructure will strengthen a material at high temperatures, just as it does at low temperatures, and the creep rate can be expected to decrease with time, as is normally the case. To check the effect of substructure on the shape of the creep curve [21], tests were made in which various amounts of substructure were introduced by straining at room temperature and recovering at $800{ }^{\circ} \mathrm{C}$ prior to creep testing at $700{ }^{\circ} \mathrm{C}$. The results are shown in figure 11. The normal concave downward shape of the creep curve was altered by the presence of the substructure. When the equilibrium substructure state was present at the beginning of the test, the creep curve was a straight line. When an excess of substructure was present, the curve became concave upward.

\section{Fracture}

While the understanding of plastic flow has gradually improved during the past quarter of a century, the concepts of fracture have remained relatively obscure. This situation is changing rapidly and it should not be long before fracture processes are well understood. Experiments with nonmetallic crystals, particularly magnesium oxide, have shed considerable light upon the failure problem. Ionic crystals are ideal for fracture studies. They develop cracks at smal1 strains; the dislocation arrays can be revealed by simple etch pit techniques; stress concentrations in such crystals cause light polarization, and this can be utilized to reveal local stress concentrations; and the crystals are transparent so the progress of growing cracks can easily be followed. The evidence now seems conclusive that cracks form as a result of dislocation interactions in regions where the motion of dislocations is impeded by some sort of barrier. In relatively pure materials, the barriers that lead to crack formation are slip bands and grain boundaries 25]. Fracture in single crystals of magnesium oxide is initiated by dislocations that have piled up at the intersections of slip bands; hence plastic flow is responsible for the nucleation of cracks in ductile ionic crystals $[25,26,27]$. Theoretical studies and experimental work have both shown that many dislocations may accumulate on a single slip plane when a barrier is present to impede the motion of dislocations. Pileups cause high local stress concentrations, and such stresses may reach values hundreds of times greater than the average stress [9]. Zener [28] in 1948 suggested that these high stresses might cause the leading dislocations to coalesce. Although dislocations normally repel each other when they are on the same slip plane, they can be forced together to form a crack nucleus in the manner shown in figure 12. After the first few dislocations have been united, coalescence becomes easier. The furmation of a microcrack does not necessarily lead to immediate failure, however, because the opening will normally extend only through the region of high stress at the end of the slip band. Then the tiny crack will cease to grow unless its length is greater than the critical value needed for spontaneous propagation. Shear stresses are effective in nucleating cracks, but the growth of a crack is clearly dependent upon the hydrostatic component of the applied stress. The extension of a crack is hindered by hydrostatic pressure [29].

The concept of crack nucleation by the coalescence of dislocations at the head of a pileup was developed quantitatively by Stroh [30]. He concluded that the stress would be high enough to cause a crack to form if $\mathrm{N}$ dislocations were piled up under an average shear stress, $\sigma$, when $\mathrm{Nb} \sigma=12 \gamma$, where $\gamma$ is the surface energy and $b$ is the Burgers vector. In deriving this equation, Stroh considered that all of the dislocations on the slip plane contributed to the nucleation of the crack. In a subsequent analysis [31] the problem was considered in greater detail, and the view was expressed that a crack is nucleated because of the interaction of a few dislocations at the head of the pile. The remaining dislocations were presumed to cause the high stress concentration necessary to collapse the leading dislocations into a crack nucleus. Microcracks of this type have been observed in crystals of magnesium oxide「26]; an example is shown in figure 13 .

Cleavage cracks on (100) type planes form in magnesium oxide as a result of interactions of dislocations moving on two mutually perpendicular slip planes. This is illustrated by the sketch in figure 14, and photomicrographs showing cracks in magnesium oxide that had been formed by this process are shown in figure 15 .

Grain boundaries can also act as barriers to dislocations and can thus contribute to crack formation. Figure 16 is a photograph taken by Westwood [32] which shows how slip interference at boundaries can cause cracking. 
Great progress has been made toward a clear understanding of flow and fracture phenomena, particularly during the past decade wherein dislocation studies made on nonmetallic crystals contributed largely to the advanced knowledge of today. As an outgrowth of such work, it has become evident that major advantages may accrue in the field of ceramics because of the research on dislocation behavior.

References

[1] A. Joffe, M. W. Kirpitschewa, and M. Lewitsky, Deformation and strength of crystals, Z. Phys. 22, 286 (1924).

[2] J. F. Nye, Plastic deformation of silver chloride. I-Internal stresses and glide mechanism, Proc. Roy. Soc. A198, 191 (1949).

[3] J. J. Gilman and W. G. Johnston, The origin and growth of glide bands in lithium fluoride crystals, Dislocations and Mechanical Properties of Crystals, John Wiley \& Sons, p.I16 (New York, 1957).

[4] J. J. Gilman, W. G. Johnston, and G. W. Sears, Dislocation etch pit formation in lithium fluoride, $\mathrm{J}$. App1. Phys. 29, 747 (May 1958).

[5] W. G. Johnston and J. J. Gilman, Dislocation velocities, dislocation densities, and plastic flow in lithium fluoride crystals, J. App1. Phys. 30, 129 (1959).

[6] A. E. Gorum, E. R. Parker, and J.A. Pask, Effect of surface conditions on room-temperature ductility of ionic crystals, J. Am. Ceram. Soc. 41, 161 (May 1958).

[7] E. R. Parker, J. A. Pask, J. Washburn, A. E. Gorum, and W. Luhman, Ductile ceramics - A high temperature possibility, J. Metals, AIME, 10, 353 (May 1958).

[8] J. Frenkel, Zur Theorie der Elastizitätsgrenze und der Festigkeit Krystallenescher Köper, Z. Phys. 37 , 572 (1926).

[9] A. Cottre11, Dislocations and Plastic Flow in Crystals, Clarendon Press (Oxford, 1953).

[10] G. I. Taylor, The mechanism of plastic deformation of crystals, Proc. Roy. Soc. 1145,362 (1934).

[11] E. Orowan, Plasticity of crystals. IV. Further foundation for the dynamic plasticity law, Z. Phys. 89, 605, 614,634 (1934).

[12] M. Polanyi, Form of lattice distortion that may render a crystal plastic, $Z$. Phys. 89, 660 (1934).
[13] L. Prandt1, Ein Gendankenmodel1 zur Kinetischen Theorie der festen Köper, Z. angew. Math. u. Mech. $\underline{8}$, 85 (1928).

[14] U. Dehlinger, Theory of recrystallization of pure metals, Ann. Phys. 5, $749(1929)$.

J. M. Burgers, Some considerations on the fields of stress connected with dislocations in a regular crystal lattice, Proc. Konink1. Ned. Akad. Wetenschap. 42, 293, 378 (1939).

[16] F. C. Frank and W. T. Read, Multiplication processes for slow moving dislocations, Phys. Rev. $\underline{79}$, 722 (1950).

[17] W. C. Dash, Report on the International Conference on Dislocations and Mechanical Properties of Crystals, John Wiley \& Sons. p.57 (New York, 1957).

Z. S. Basinski, Thermally activated glide in face-centered cubic metals and its application to the theory of strain hardening, Phil. Mag. 4, 393 (1959).

[19] A. Seeger, S. Mader, and H. Kronmüller, Theory of work-hardening in FCC and HCP single crystals, Electron Microscopy and Strength of Crystals, John Wiley \& Sons (New York, 1962).

[20] W. G. Burgers and J. M. Burgers, Second report on viscosity and plasticity, Proc. Acad. Sci. Amsterdam, P.200 (1938).

[21] E. R. Parker, Modern concepts of flow and fracture, Trans. ASM $\underline{58}$, $52(1958)$.

[22] W. M. Lomer, A dislocation reaction in the face-centered cubic lattice, Phil. Mag. 42, 1327 (1951).

[23] Betsy Ancker and E. R. Parker, Quantitative substructure and tensile property investigation of nickel alloys, Trans.AIME 200, 1155 (1954).

[24] J. E. Dorn, Some fundamental experiments on high temperature creep, J. Mech. and Phys. Solids $\underline{3}, 85$ (1954). 
[25] Fracture, Ed. B. L. Averbach, D. K. Felbeck, G. T. Hahn, and D. A. Thomas, John Wiley \& Sons (New York, 1959).

[26] R. J. Stokes, T. L. Johnston, and C. H. Li, Crack formation in magnesium oxide single crystals, Phil. Mag. $\underline{3}, 718$ (1958).

[27] J. Washburn, A. E. Gorum, and E. R. Parker, Cause of cleavage fracture in ductile materials, Trans.AIME $\underline{215}, 230$ (1959).

[28] C. Zener, Fracturing of Metals, ASM (Cleveland, Ohio, 1948).

[29] P. W. Bridgeman, Studies in Large Plastic Flow and Fracture, McGrawHill Book Co. (New York, 1952).
[30]

A. N. Stroh, The formation of cracks as a result of plastic flow, Proc. Roy. Soc. (London) 르르, 404 (1954).

[31] A. N. Stroh, The formation of cracks in plastic flow II, Proc. Roy. Soc. (London) A232, 548 (1955).

A. R. C. Westwood, private communication.

[33] K. Llicke and H. Lange, The shape of the solidification curve of highpurity aluminum crystals and the formation of deformation curves, Z. Metallk. 43, 55 (1952).

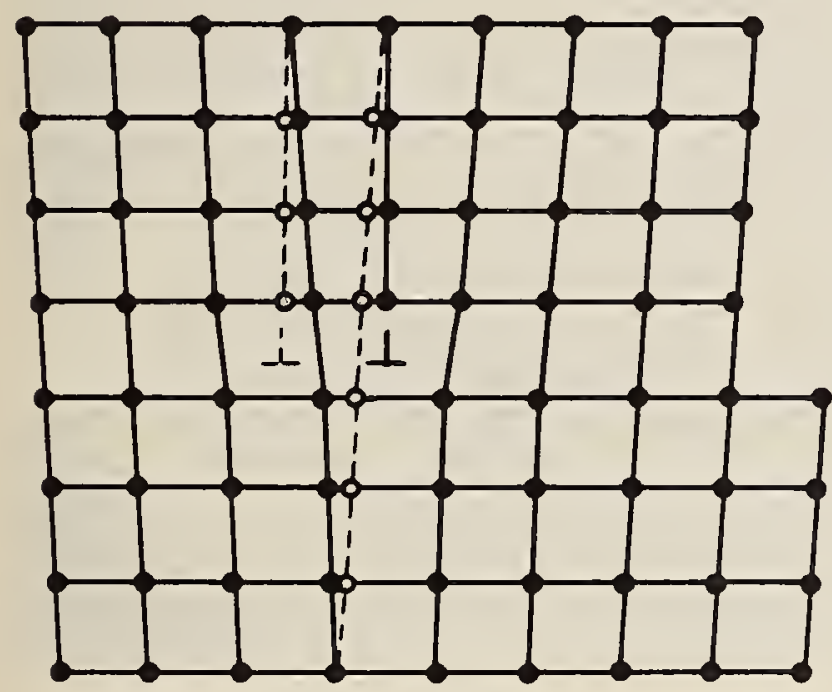

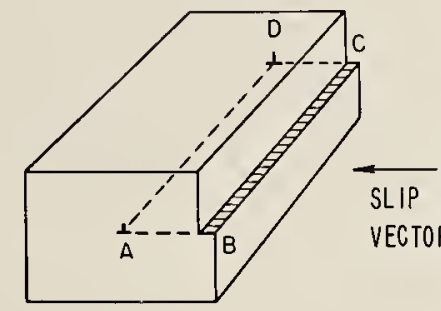

(a)

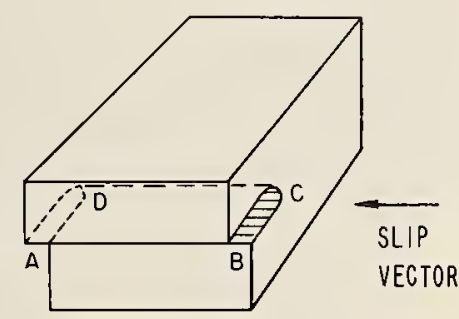

(b)

FIGURE 2. Sketches showing how slip can occur in a crystal by movement of (a) an edge dislocation from front to back or. (b) a screw dislocation from right to left.

FIGURE 1. Sketch of section through a simple cubic lattice showing nature of structural defect called an edge or line dislocation.

FIGURE 3. Sketches showing how a dislosation can be generated by the action of a stress wizen a crystal contains a grown-in dislocation loop, part of which lies in a slip plane.
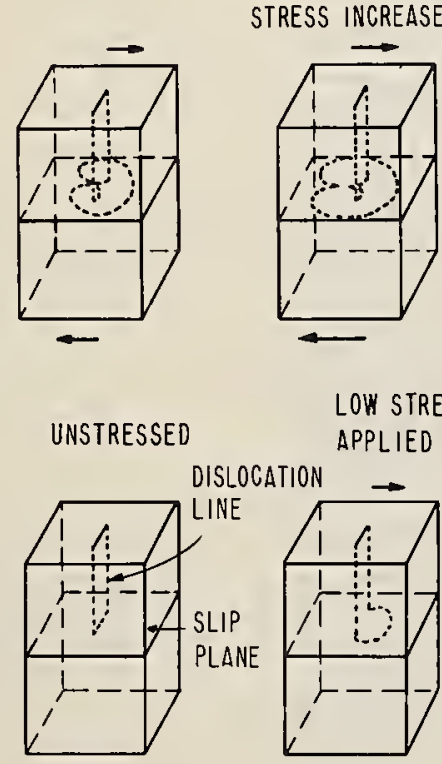

LOW STRESS APPLIED

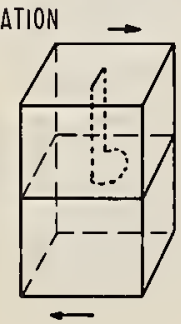

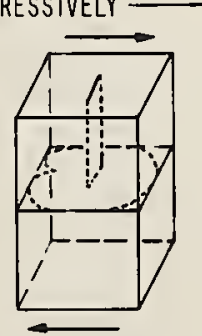

STRESS INCREASED SLIGHTLY

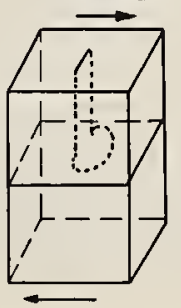

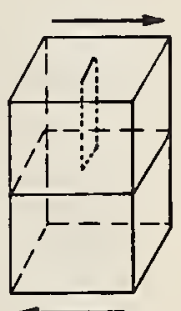

STRESS INCREASED MORE

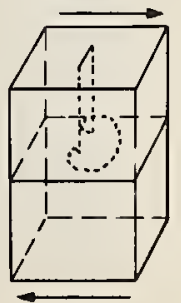



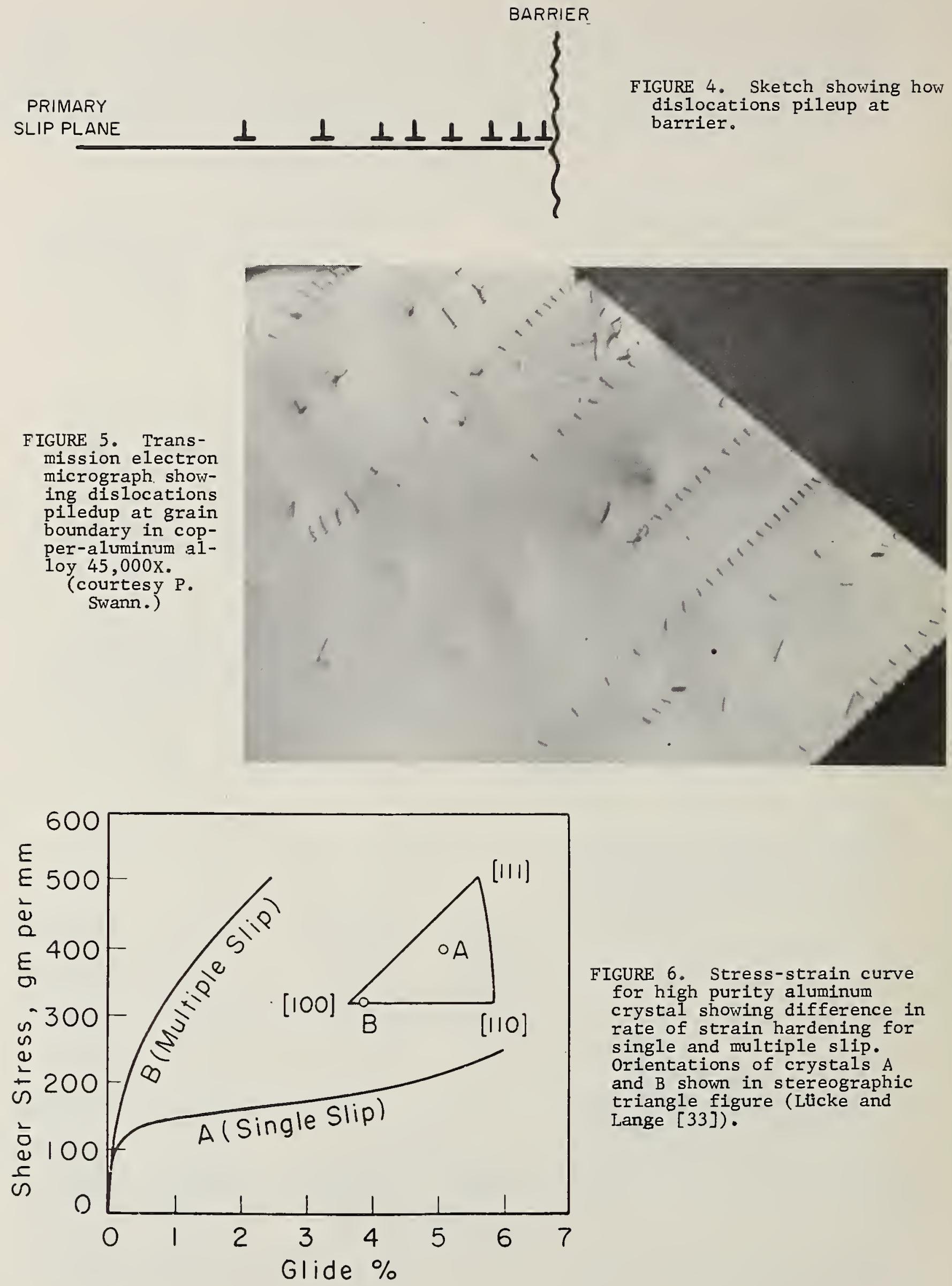

FIGURE 6. Stress-strain curve for high purity aluminum crystal showing difference in rate of strain hardening for single and multiple slip. Orientations of crystals A and $B$ shown in stereographic triangle figure (Llicke and Lange [33]). 
FIGURE 7. Transmission electron micrograph showing dislocation tangles in molybdenum $37,500 \mathrm{x}$. (courtesy G. Thomas.)
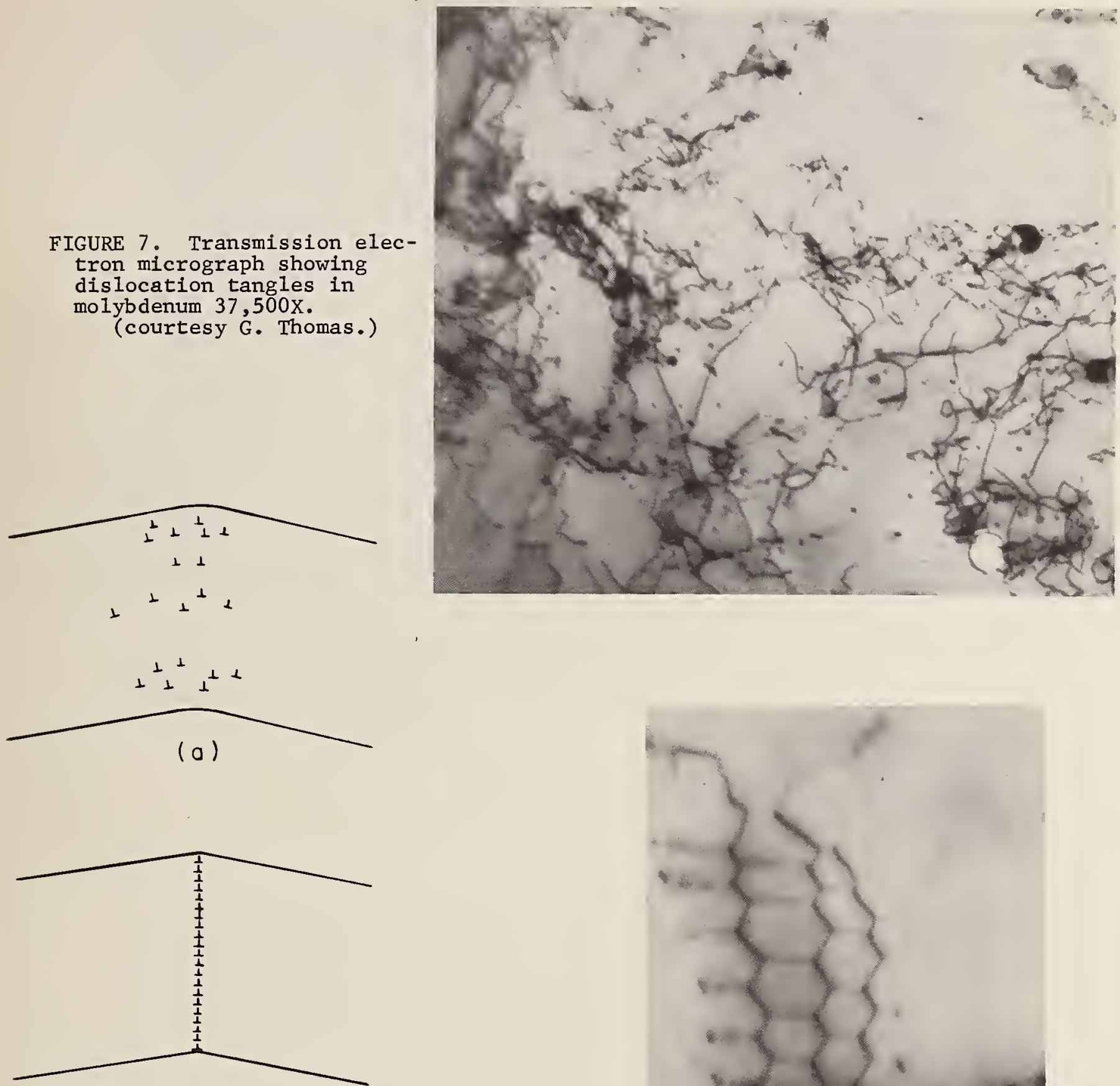

(b)

FIGURE 8. Sketch illustrating change in structure of dislocation boundary produced by heating. (a) Before heating; (b) after heating to a temperature high enough for rapid dislocation climb.

FIGURE 9. Transmission electron micrograph showing dislocation subboundaries formed by heating deformed molybdenum to a high temperature $75,000 \mathrm{x}$.

(courtesy G. Thomas.)

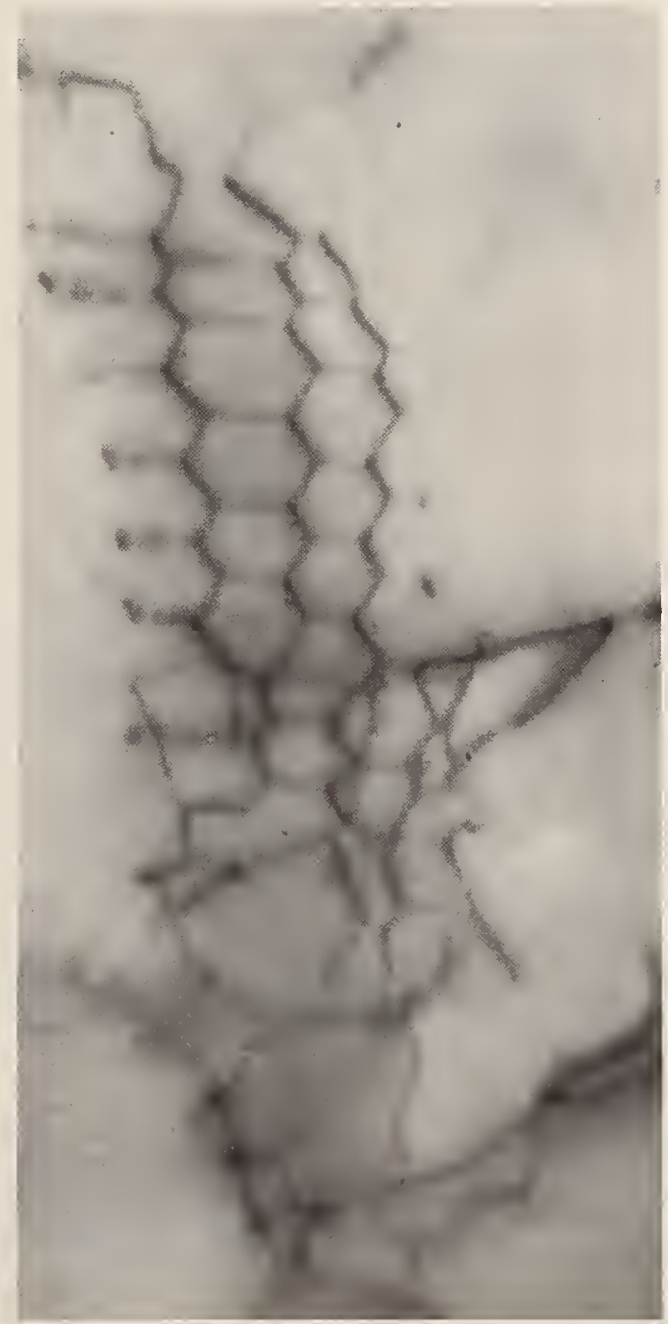




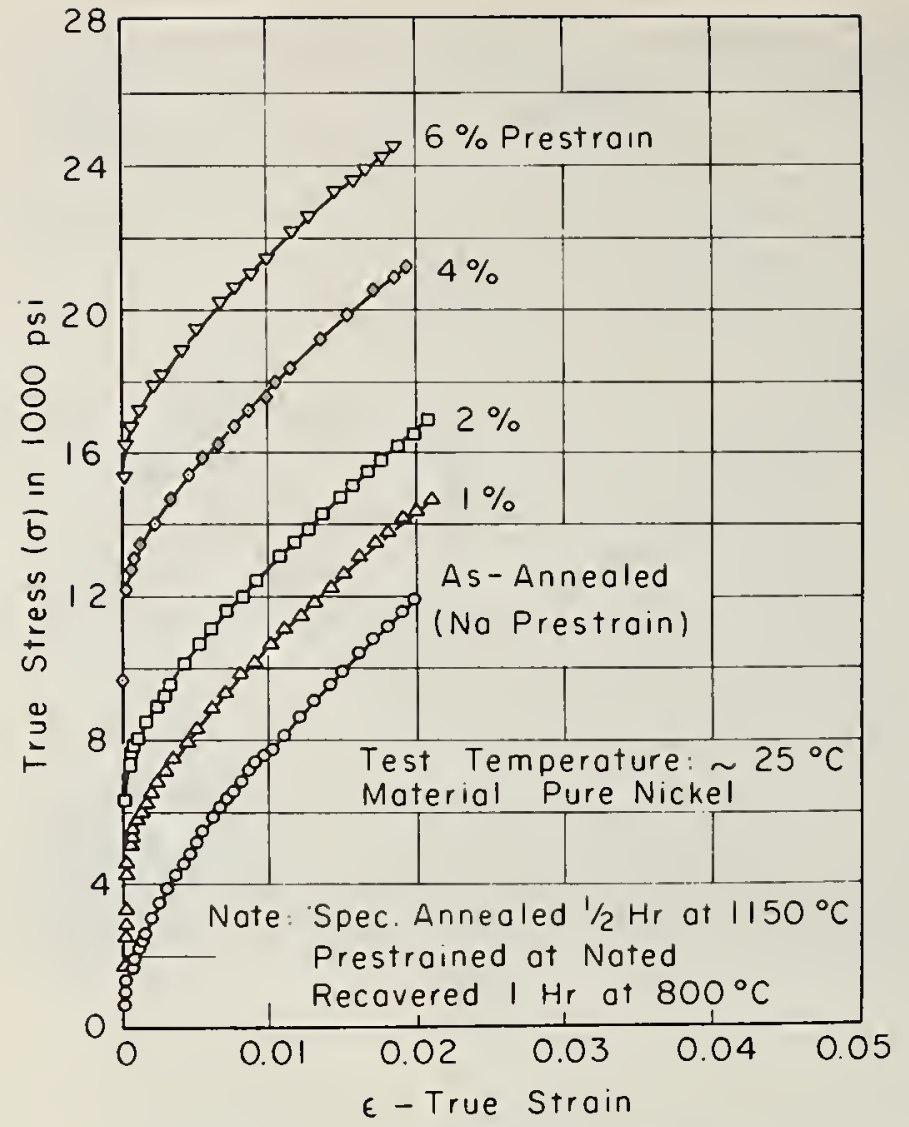

FIGURE 10. Effect of dislocation boundaries developed within the grains of polycrystalline nickel (by a prestrain and anneal treatment) on the stress-strain curve.

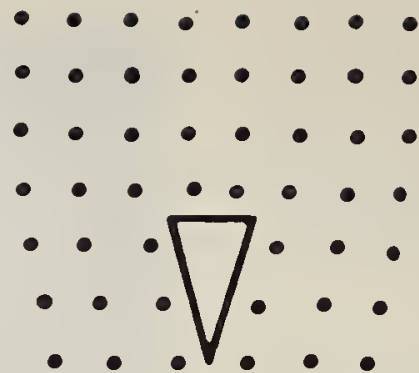

(a)

COALESCENCE OF TWO DISLOCATIONS
COALESCENCE OF THREE DISLOCATIONS

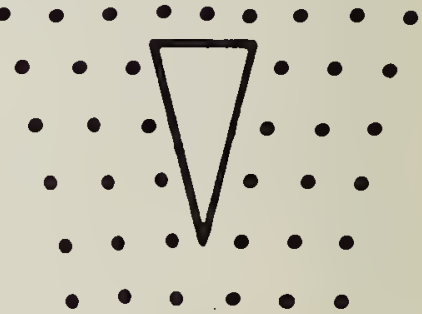

FIGURE 12. Sketch illustrating how dislocations can coalesce to form a crack nucleus.

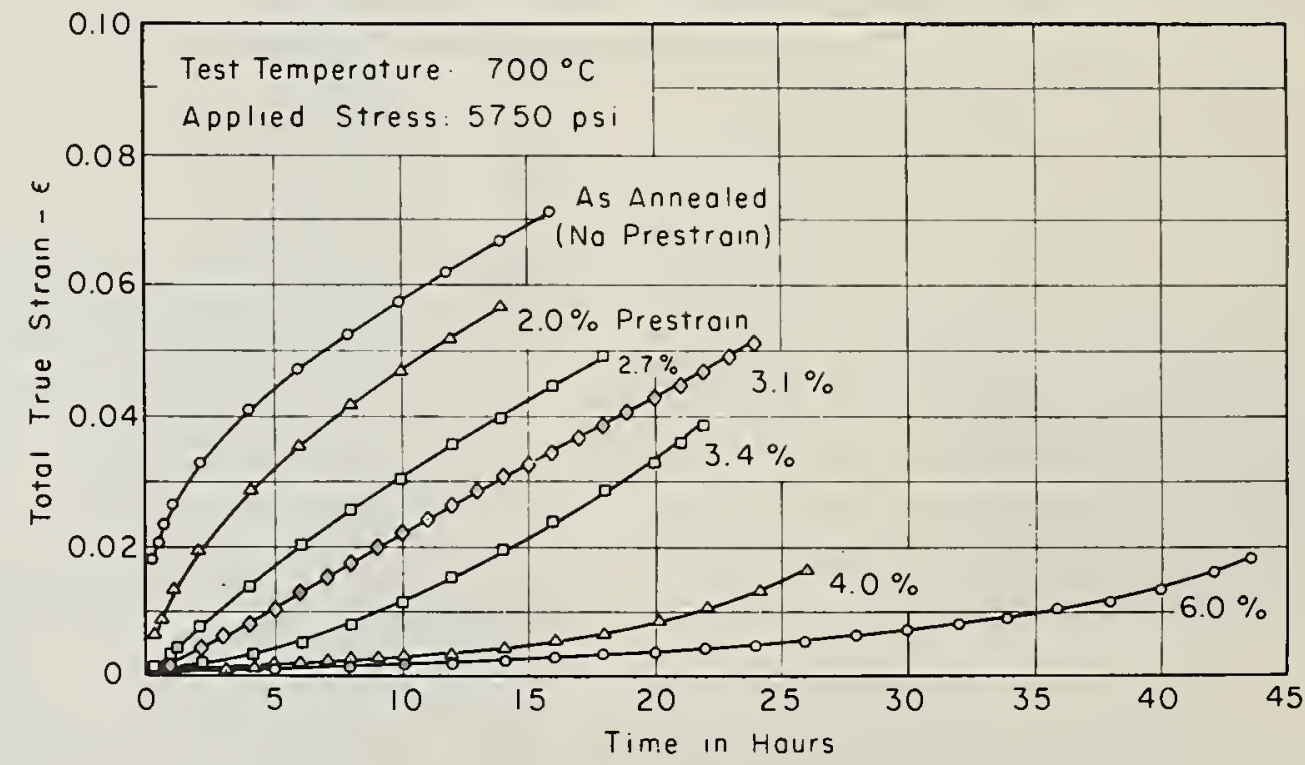

FIGURE 11. Effect of substructure introduced by prestraining indicated amounts and annealing at $800{ }^{\circ} \mathrm{C}$ on shape of creep curve for high-purity nickel. 


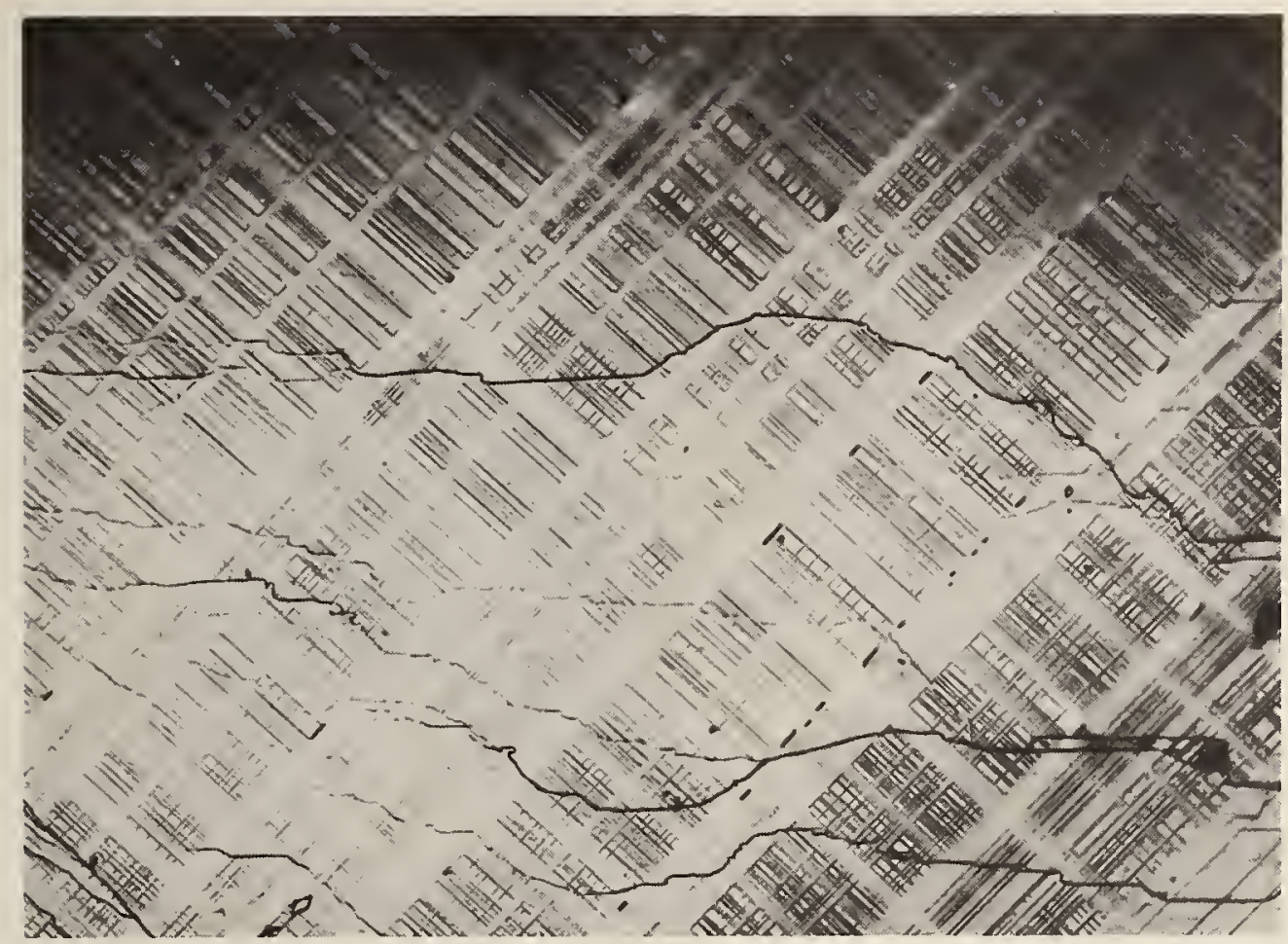

FIGURE 13. Photomicrograph of deformed magnesium oxide showing microcrack formation at intersections of slip bands, 187X (Stokes, Johnston, and Li [26]).

FIGURE 14. Sketch showing how dislocations pileup at intersection of slip bands can lead to cleavage cracks. Bands $A C$ and $B C$ intersect at point $C$ where crack nucleation occurs.

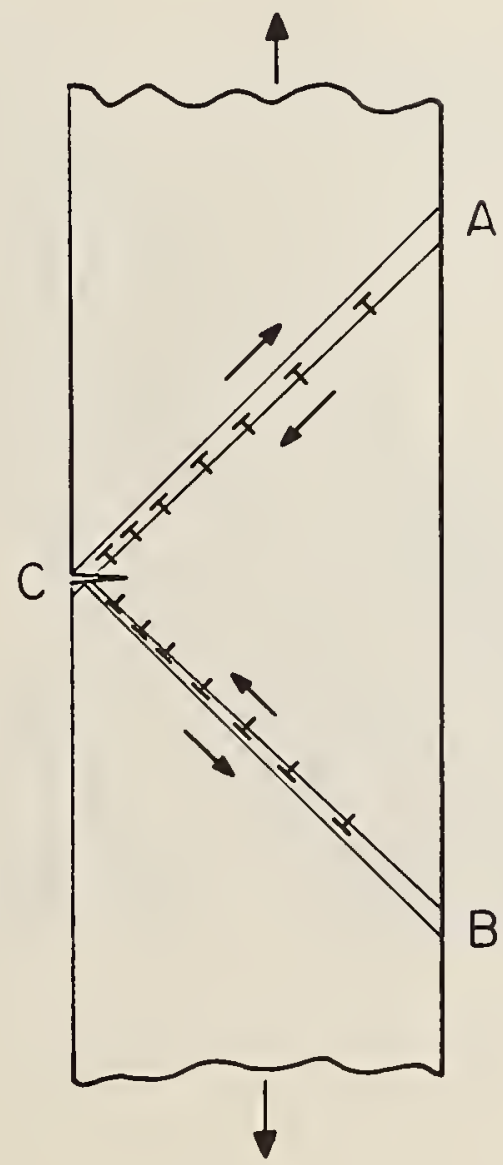


FIGURE 15. Photomicrographs showing examples of cleavage cracks forming at intersections of slip bands in magnesium oxide single crystals (a) and (b) 750X; (c), (d), and (e) 300X.

(a)

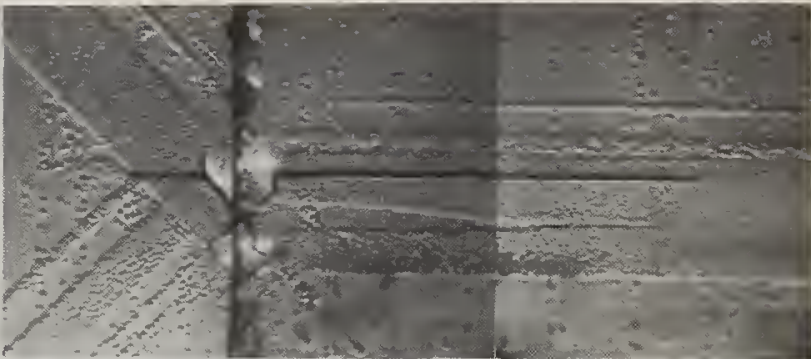

(b)

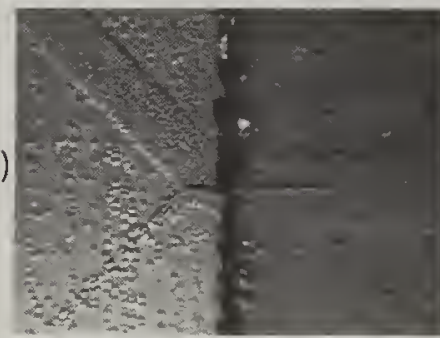

(c)

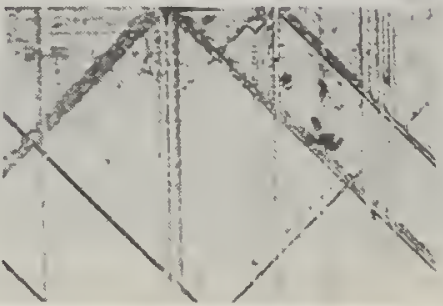

(d)

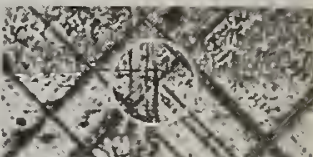

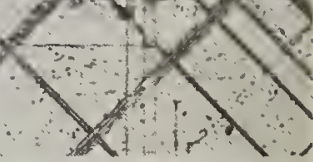

(e)

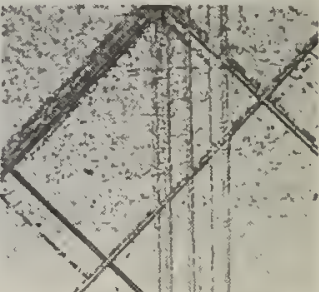

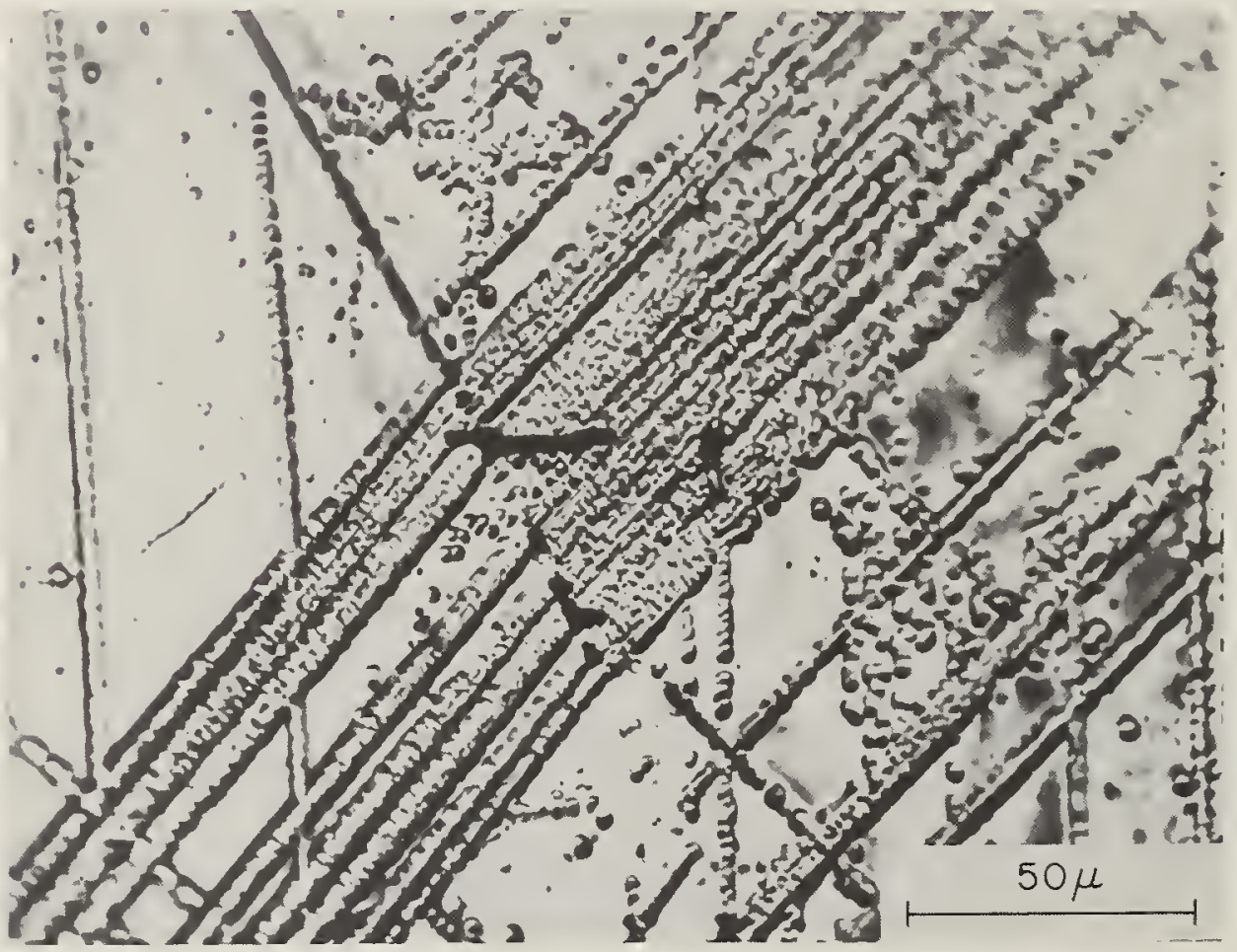

FIGURE 16. Photomicrograph of polycrystaline magnesium oxide showing examples of microcracks formed where slip bands intersect grain boundary (Westwood [32]). 
Roland de Wit

The paper starts by pointing out the nature of a dislocation as a region of nonuniform slip. The emphasis is on a pictorial representation to clarify the three dimensional aspects of the theory. Examples of dislocations in the simple cubic crystal structure are presented, such as the edge, screw, and bent dislocation. A definition of the dislocation with its associated Burgers or slip vector is given in terms of a geometrical construction. There is a discussion of the two kinds of dislocation motion: (1) glide, which is progressive slip, and (2) climb, which is nonconservative motion. The nature of the force on a dislocation is described. Finally, kinks and jogs are considered; these are offsets in a dislocation line which result from nonuniform motion or dislocation intersections.

\section{Introduction}

This symposium will introduce the idea of a dislocation as a physically observed entity that plays a very important role in the macroscopic mechanical behavior of crystalline solids. The first paper gave a broad review of the field of dislocations. This paper will serve to introduce a clear and descriptive definition of a dislocation. This should be helpful when trying to determine whether an observed imperfection of unknown origin is a dislocation or not. The paper following this one will present experimental evidence for believing in the existence of dislocations, and it will treat in detail some of the techniques for observing individual dislocations.

Though some readers may already be quite familiar with the field, this paper is addressed primarily to those for whom the concept of a dislocation is a new one. That is, we shall assume that most readers are wholly unacquainted with the theory of dislocations. The paper will be concerned primarily with the geometry of dislocations. It emphasises a simple physical way of thinking based on pictures, which, it is hoped, will help clarify the three dimensional concepts that are essential for an understanding of dislocation theory. To illustrate the crystallographic features of a dislocation the simple cubic crystal structure is used, where atomic arrangements in three dimensions are relatively easy to visualize. The justification for using a simple cubic for introductory and illustrative purposes is twofold: (1) many calculations based on it lead to fairly good results, and (2) dislocation theory in other types of crystal structures is a simple extension of the theory in the simple cubic. This is so because the concept of a dislocation as such is simply a certain type of imperfection in a lattice, irrespective of the particular type of lattice under consideration. A crystal is called perfect if the atoms are arranged in a regular pattern based on a lattice. By an imperfection is meant a small region where the regular pattern breaks down and some atoms are not properly surrounded by their neighbors. To study the perfect crystal and the crystal imperfection is the primary interest of a field called solid state physics. The effort in this field is about equally divided between the two; the perfect crystal is usually studied by quantum mechanical methods, while classical physics is normally used for studying the crystal imperfection.

\section{The Concept of a Dislocation as Localized Slip}

The plastic deformation of crystalline solids is highly anisotropic and inhomogeneous. It has been found that deformation does not take place by a uniform shear of the crystal, but rather by the atoms sliding over one another along well-defined crystallographic planes, like cards in a deck. This is shown in figure 1 . The planes on which the slip occurs (slip planes) are usually the close packed planes (those planes which are most densely packed with atoms). The directions in which the slip occurs (slip directions) are usually the close packed directions in the slip planes (the directions of most densely packed atom rows). The magnitude of the slip is usually many interatomic distances in the slip direction. However, it can be considered to be made up of units equal to a primitive translation vector of the lattice on which the crystal is based (slip vectors). There is one unit of slip on a slip plane if every atom on one side of that plane has moved into position originally occupied by its nearest neighbor in the slip direction.

The basic idea of dislocation theory is that slip is a local process, and that plastic deformation occurs by the gradual extension of regions of local siip and not by simultaneous and uniform slip over the whole slip plane. It is apparent that there must be boundaries separating the slipped regions from the unslipped. These boundaries are called dislocations and slip proceeds by the motion of these disiocations. Figure 
2 shows how the motion of the boundary of a slipped region extends the slip through the crystal, until it finally covers the whole slip plane.

\section{Edge Dislocation}

When the boundary between the slipped and unslipped regions lies perpendicular to the slip direction this boundary is called an edge dislocation (or Taylor-Orowan dislocation). This is shown in figure 3. Unit slip has occurred over the area $A B C D$ of the slip plane. The boundary $C D$ of the slipped area is the dislocation. Figure 4 shows the atomic planes above and below the slip plane, which is parallel to the plane of the figure, the open circles represent atoms just above the slip plane, and the solid circles, atoms just below. Notice that at the dislocation CD three rows of open circles must join onto two rows of solid circles; the middle row of open circles is therefore the edge of an extra atomic plane.

If the crystal is viewed along the dislocation line $C D$, we see the picture shown in figure 5, which is a photograph of a model of an edge dislocation. The model shows that the dislocation is the edge of an incomplete atomic plane; hence, the name edge dislocation. The center (or core) of the dislocation is a region of severe atomic misfit, where atoms are not properly surrounded by their neighbors in the simple cubic pattern. The atoms at the edge of the incomplete atomic plane do not even have the right number of nearest neighbors. The exact arrangements of atoms at the core is not known.

Across the slipped region to the left of the dislocation the atoms are in register again across the slip plane, though slightly sheared. This is so because the slip vector equals a translation vector of the lattice. Unit slip does not disturb the crystal perfection across the slip plane. Therefore a dislocation is a structural line imperfection. Observe that, if the dislocation were to move across the slip plane (from one side of the crystal to the other), the crystal would deform.

Figure 5 shows that an alternative way of forming an edge dislocation is to remove half a plane of atoms below the slip plane or insert an extra half plane above. This view helps to visualize another way of forming a dislocation namely, by mass transport. In real crystals this might occur by the diffusion of vacancies or interstitial atoms.

Figures 4 and 5 show that the concept of a dislocation (or any other imperfection of crystal structure) is really a shorthand for describing the positions of a large number of atoms - it is much easier to describe crystals by listing the imperfections than by giving the position of every atom.

\section{Screw Dislocation}

If the boundary line of the slipped region lies parallel to the slip direction, as shown in figure 6 , it is called a screw dislocation (or Burgers dislocation). Again, unit slip has occurred over the area $A B C D$ and the boundary $A D$ is the dislocation. Figure 7 shows the arrangement of atoms. As before, the plane of the drawing is the slip plane; the open circles represent atoms directly above the slip plane, and the closed circles, atoms directly below.

Viewing the crystal along a line parallel to $C D$, we see the picture shown in figure 8 , a model of a screw dislocation. Again, the core of the dislocation is a region of severe atomic misfit. However, the atoms at the core do have the right number of nearest neighbors. The atoms nearest to the dislocation are arranged on a regular helix; hence, the name screw dislocation. In fact, the whole crystal is a single atomic plane in the form of a helicoid, or spiral ramp. This can be seen by carefully studying figure 8 . The distortion around the screw dislocation is a rotationally symmetric pattern. ${ }^{1}$ The strain is pure shear. The slip plane is therefore not uniquely defined. The same screw dislocation in figure 6 can be produced by slip on any plane ending on $A D$.

The method of representing the distortion in these figures makes the dislocation appear to have a unique slip plane. This is related to the following interesting property of a screw dislocation: wherever it intersects the surface of the crystal there is the beginning of an atomic step on the surface. This incomplete step is typical of a screw dislocation. A different slip plane would leave the form of the screw disloca-

${ }^{1}$ A symmetrical screw dislocation might be able to lower its energy by spreading out in one slip plane; the resulting asymmetric screw will move most easily on that slip plane. 
tion unaltered, but would change the direction of the step on the surface.

The screw dislocation may be right-handed, as in these figures, or left-handed.

\author{
Arbitrary Dislocation on Slip Plane
}

A slipped region can be bounded by an edge and a screw dislocation as shown in figure 9. Here unit slip has occurred over the area $A B C D$ and the boundary $C D$ is an edge disiocation, while $A D$ is a screw dislocation. The two different types of dislocations join each other in a right angle at D. Another way of looking at this situation is to say that the dislocation changes its character from edge to screw as it bends through a right angle. Figure 10 shows the atoms just above (open circles) and just below (solid circles) the slip plane $A B C D$. Note that the atomic arrangement along $C D$ is similar to that along $C D$ in figure 4 while the arrangement along $A D$ is similar to that along $A D$ in figure 7 . Figure 11 is a model of this dislocation viewed along the line $\mathrm{CD}$. Note that the left hand part of the model is similar to figure 8 and the front part similar to figure 5.

There is no reason to expect that an edge and a screw dislocation will join each other in an abrupt right angle. Rather, the dislocation will tend to curve gradually, as shown in figure 12. A detailed drawing of the arrangement of atoms near the slip plane for this curved dislocation is shown in figure 13. The atomic disorder varies continuously along the curve. At $\mathrm{C}$ the dislocation is in the edge orientation and the atomic arrangement is the same as at $C$ in figure 10; at $A$ it is in the screw orientation, the same as at $A$ in figure 10. Between $C$ and $A$ the dislocation changes its character continuously from edge to screw as it turns through a right angle. Follow the extra plane of atoms starting at $C$ into the crystal and observe that, as the dislocation curves and ceases to be pure edge, the extra plane ceases to be "extra," but joins onto an atomic plane below the slip plane. This curved dislocation shows that the edge and screw refer not to properties of the whole dislocation, but to the local orientations. Another way to view this dislocation is to observe again that it is the boundary of the slipped area $A B C$. However, slip has proceeded by different amounts on different parts of the slip plane.

A dislocation that is neither pure edge nor pure screw is called a mixed dislocation. This type of dislocation may be considered to be made up of elements of edge and screw dislocation. The amount of mixture of each type is specified by giving the angle between the dislocation line and the slip direction. For example, we could discuss the properties of a $60^{\circ}$ dislocation.

Even if plastic deformation occurs on a single slip plane, the slipped area may have an arbitrary shape on that plane. Its boundary is then an arbitrary plane curve, in other words, a dislocation of continuously changing orientation and character.

Are there any limitations on the curvature of a dislocation? There is good reason to expect that dislocations will straighten out as much as possible. This will reduce the amount of elastic strain in the crystal. That is, a dislocation tries to reduce its energy by being as short as possible. The effect is as if there is a line tension on the dislocation.

\title{
Arbitrary Slip Surface
}

The dislocations discussed above all lie in a single plane; that plane contains the slip vector and is therefore the slip plane. However, the most general slipped area is a surface which everywhere contains the slip vector.

An example of a segmented slip surface is shown in figure 14. Here the slipped area consists of two Planes, ABCD and DEFA, which both contain the slip vector. Therefore the intersection $A D$ is parallel to the slip vector. The dislocation line CDE is shaped like an L; it is everywhere perpendicular to the slip vector and therefore edge. If we look down the line ED from above and concentrate our attention on the slip plane $A B C D$, we see the arrangement of atoms shown in figure 15. The section of dislocation $C D$ (in the plane of the figure) and the section DE (normal to the figure) are both edges of the same extra plane. This shows, as before, that an alternative way of forming this dislocation is to insert an extra quarter plane of atoms into a perfect crystal. Note also that the atomic arrangement at $C$ in figure 15 is similar to that at $C$ in figure 4. 
A more general slip surface is shown in figure 16. It contains the slip vector everywhere, but otherwise follows the arbitrary step $A B$ on the surface. The slipped area is $A B C D$ and $C D$ is the dislocation. The curve $C D$ is everywhere perpendicular to the slip vector and therefore an edge dislocation. Again, this dislocation can be obtained by slipping into the crystal an incomplete plane of atoms, whose incomplete edge follows the curve $\mathrm{CD}$.

More generally, slip may proceed by different amounts on different parts of the slip surface as shown in figure 17. Here again, the slip surface ABCD contains the slip vector everywhere and follows the arbitrary step $A B$, but now terminates in the arbitrary curve $\mathrm{CD}$, the dislocation. The dislocation has also some screw character in addition to the edge character of figure 16. The construction of figure 17 allows us to form by slip a dislocation following any arbitrary curve in a crystal. One can therefore always visualize dislocations in terms of slip without loss of generality.

However, on an atomic scale, the most general slip surface is made up of segments of slip planes, which intersect along lines parallel to their common slip vector. Thus we can resolve any dislocation into straight line segments, which may only be a few atoms long.

In the discussions so far the dislocation was the boundary of a slipped region on a slip surface. The dislocation is defined by the slip vector and the dislocation line. However, we have also seen that dislocations can be created by inserting incomplete atomic planes, rather than pure slip. In the next section we shall consider a more general definition of a dislocation in terms of a more general surface, which however will not necessarily be the slip surface.

\section{General Definition of a Dislocation ${ }^{2}$}

A general definition of a dislocation will now be given in terms of a geometrical construction. We can visualize a dislocation by imagining that it is made as follows: Start with a perfect crystal. Make a cut in it along a surface $S$ bounded by a closed curve $C$, as shown in figure 18. The curve may intersect the surface of the crystal in special cases. The positive direction of the normal $\mathrm{n}$ to the surface $\mathrm{S}$ is related to the direction of the curve $C$ by the right-hand screw Fule. Next, imagine that the portion of the crystal on the negative side of the surface $S$ is displaced by a vector distance $b$ with respect to the positive side, $b$ being the same over the entire surface. The vector $\underline{b}$ is called the Burgers vector and equals a primitive translation vector of the lattice (slip vector). Examples of it are shown in figure 19 and 20 , which will be discussed below. Unless the surface $S$ contains b (a slip surface), the relative displacement of the crystal on each side of the cut either produces a gap or causes the two sides to overlap. Imagine that atomic layers are added or removed to produce continuity. The end result is a general dislocation line along the curve $\mathrm{C}^{3}$

Since $b$ is a lattice vector the atoms are in register again over all the surface $S$, except at the region near the dislocation $C$, where there is appreciable disregistry and strain. The dislocation is characterized by the curved line $\mathrm{C}$ and the Burgers vector $b$; it is independent of the surface $S$. Two results that follow directly from this definition are that (I) a dislocation line cannot end inside a crystal, and (2) the Burgers vector of a dislocation is constant.

Figure 19 shows how the above general construction applies to the particular case of an edge dislocation. Part (a) shows the three dimensional relationships similar to those in figure 3. Part (b) shows a plane of atoms normal to the dislocation line. The symbol $\perp$ (resembling an extra half plane) denotes the dislocation. The surface $S$ of the definition is taken to be the slipped region of the slip plane. If the dislocation runs out of the paper in (b), then the normal n points down. The upper left hand part of the crystal, which is also the negative side of $S$, has been displaced by a Burgers vector (slip vector) with respect to the lower left hand part.

\footnotetext{
${ }^{2}$ Other definitions are often used that are equally satisfactory; for example, in terms of a Burgers circuit.

${ }^{3}$ This construction gives a definite direction to the Burgers vector in relation to the direction of the dislocation. This relationship is the reverse of that adopted by W. T. Read, Jr. The literature of this field is about evenly divided between the two choices of the sign of the Burgers vector.
} 
Figure 20 shows an example where the surface $S$ is taken to be the extra half plane above the dislocation. The normal nn now points to the left in (b). In order to perform the construction in this case, a cut is made along $S$ and the extra half plane inserted. Therefore it is necessary to wedge the crystal apart, displacing the upper right hand part of the crystal, which is the negative side of $\mathrm{S}$, by a Burgers vector with respect to the upper left hand part.

In both the above particular cases we see that the crystal is in register again across the surface $S$ after the introduction of the dislocation. Also, we see that the atomic arrangement is the same at the core of the resulting edge dislocation, independent of the choice of the surface $\mathrm{S}$.

This section has emphasized a sign convention. A consistent use of signs and directions is important in the mathematical development of dislocation theory. However, for the rest of this paper we shall treat directions in space from an intuitive rather than a mathematical point of view.

\section{Structure of a Dislocation}

This section mentions in a qualitative way what has become known as the "dislocation core problem." This is the question of what is the form or fine structure of a dislocation, i.e., the detailed atomic arrangement at the center or core of the dislocation. Since the distortion is so severe, it is not possible to use classical elasticity theory, which can be applied successfully to small deformations of crystals. It is necessary to take into account the periodic nature of the crystal. Several approximate approaches have been made, based on the idea of interatomic forces. The simplest of these is the one by $R$. Peierls, who assumed a sinusoidal variation of shear stress across the slip plane. It led to what is known as the Peierls-Nabarro model of a dislocation. Though this model is used extensively in the field, any results of it should be regarded with caution because of the unrealistic sine law assumption.

One of the most important results to come out of a dislocation core calculation is the dislocation width. This is the area on the slip plane where the atoms are out of register by more than a certain amount, which is conventionally taken as one-half the maximum shear strain. Usually it amounts to a few Burgers vectors. Its major importance is in connection with dislocation glide to be covered next.

\section{Dislocation Motion}

A dislocation is a configuration of atoms that can move through the crystal. motion of a dislocation is a convenient shorthand for describing the motions of a large number of atoms; each atom moves only a fraction of an interatomic distance as the dislocation configuration moves through many atomic spacings. Moving dislocations are responsible for plastic deformation. A dislocation can move either in its slip plane or normal to it. Motion on the slip plane (or surface) simply enlarges the slipped area; since only slip is involved, the motion is called slipping or gliding, or just glide. Hence the name glide plane is sometimes used for slip plane. Motion normal to the slip plane is called climb.

Figure 21 shows glide of an edge dislocation by one interatomic spacing. The solid circles represent the positions of the atoms before motion, the open circles, the positions after. The motion of the dislocation involves only a minor rearrangement of the atoms. Therefore a dislocation is highly mobile. It is easy to see why the stress required to move a dislocation is at least several orders of magnitude smaller than the stress required to shear one entire plane of atoms simultaneously over another in a perfect crystal. Consider the shear on the slip plane. On the unslipped side of the dislocation, the atoms want to return to the perfect unslipped state; on the slipped side, the atoms want to go on to the completely slipped state. The atom at the center of the dislocation is in dead-center position. When the dislocation is moved from a symmetric position, the work done against the interatomic forces on one side of the center equals the work done by the interatomic forces on the other side. Thus the dislocation moves under zero applied stress. However, before the dislocation can move to the next symmetric position, it has to go through asymmetric positions, where the interatomic forces do not balance out and an applied stress is needed to move the dislocation. The same general discussion also applies to glide of a screw dislocation.

Figure 22 shows climb of an edge dislocation by one interatomic spacing. Again the solid circles represent the positions before motion, the open circles, after. The motion results in the extra half plane of atoms extending down to the next lower slip plane; hence there is one more row of atoms. The motion therefore requires some meth- 
od of adding atoms. If the dislocation moved up, atoms would have to be removed from the edge of the extra plane. So climb requires mass transport. The climb in figure 22 could take place in two ways: (1) Interstitial atoms could diffuse to the dislocation and join the extra half plane, or (2) atoms from the surrounding crystal could join the extra plane, leaving lattice vacancies to diffuse away. Climb in the other direction, when atoms are removed, could also occur in two ways: (1) Vacancies could diffuse to the dislocation and remove atoms from the extra half plane, or (2) atoms could break loose from the extra half plane, become interstitial atoms and diffuse away. Thus an edge dislocation is both a source and a sink for both vacancies and interstitial atoms. Climb is often called nonconservative motion (with reference to the conservation of mass), since atoms must be added or removed from the extra half plane. Glide is called conservative motion, because it does not require any change in the number of atoms at the dislocation core.

\section{Resistance to Glide}

This section considers the resistance to glide or plastic resistance that a crystal presents to motion of a dislocation. Figure 21 showed how the atoms move when the dislocation moves one interatomic spacing. The atomic misfit varies periodically with the period $\underline{b}$ as the dislocation moves; the configuration repeats itself every time the dislocation moves a Burgers vector. The energy of atomic misfit varies with the same period. When the dislocation is in either of the two positions shown in figure 21 , the configuration of atoms is symetric. The misfit energy is then a minimum. ${ }^{4}$ Between the minima in energy, there are maxima, which provide the resistance to dislocation motion. The theoretical stress to move a dislocation over this energy hump is a sensitive function of the dislocation width. This stress is often called the Peierls force. It can be shown that the energy and hence the plastic resistance decreases as the width of the dislocation increases. We see now the importance of a core calculation to estimate the theoretical strength of crystals with dislocations. The same arguments about resistance to glide also apply to the screw dislocation.

\section{Force on a Dislocation}

A moving dislocation can cause the crystal to yield under an applied stress. Therefore, we can say that the applied stress produces a force on the dislocation. However, this concept of force is different from the familiar one of Newtonian force on a mass. It is, rather, the force on a geometrical configuration in a crystal, to which we have given the name dislocation. So the force on a dislocation should not be confused with the force on an atom. The two are different, as will be shown below.

Figures 21 and 22 show the type of stresses that are responsible for dislocation motion and forces on dislocations. The glide in figure 21 takes place as a result of the applied shear stress $\tau$ on the upper and lower faces of the crystal. The shear stress on the left and right faces are necessary to keep the crystal in equilibrium. The stress is transmitted through the crystal and produces the same shear stress $T$ on the slip plane. It is seen that the stress results in forces on the atoms that make them move in the direction of the applied stress; to the right in the upper part of the crystal, and to the left in the lower part. The dislocation, however, moves only to the right. This shows that the type of shear stress shown in the figure produces a force to the right on the type of dislocation shown.

Figure 22 shows that climb takes place as a result of an applied tensile stress $\sigma$, which again propagates and produces the same tensile stress on the extra half plane

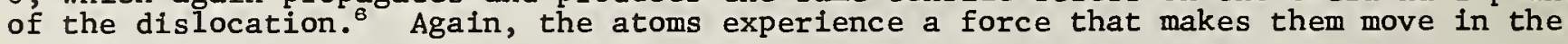

\footnotetext{
${ }^{4}$ There is another symmetric position, also with a minimum in the energy, at a position halfway between the two shown in figure 21 .

${ }^{5}$ The arguments in this section apply to dislocations lying in close packed directions. The simple cubic crystal structure is a special case where the pure edge dislocation does lie in a close packed direction. In other structures a screw will always lie in a close packed direction, but an edge usually does not. It can be shown that the resistance to motion is greatest for a dislocation in a close packed direction.

${ }^{6}$ The faces of a crystal do not necessarily run parallel to the slip planes or other special planes inside the crystal. An applied tensile stress may produce a shear stress on certain planes in a crystal and vice versa. Both glide and climb can therefore take place under both shear and tensile stress.
} 
direction of the applied stress; to the left in the left part, and to the right in the right part of the crystal. The dislocation now moves down, so that the tensile stress shown produces a force down on the type of dislocation shown. A compressive stress in figure 22 would make the dislocation move up, hence produce a force up on the dislocation. This last result shows that reversing the applied stress reverses the force on the dislocation.

\section{Macroscopic Deformation from Dislocation Motion}

This section discusses how the crystal deforms macroscopically as a dislocation moves through it. Figure 23 shows four successive positions of an edge dislocation as it glides all the way across a crystal, beginning with a perfect unslipped crystal and ending with a perfect slipped crystal. In the final state (d), the upper half of the crystal is offset by one interatomic spacing b relative to the lower half. As the dislocation moves and the crystal deforms, the applied stress $T$ does work. When the dislocation moves all the way across the slip plane, the work done is $\mathrm{Tb}$ per unit area (the upper surface is offset by $b$; the force on it is $T$ per unit area). The force (per unit length) on the dislocation is defined as the work done when a unit length of dislocation moves a unit distance. So the force on the dislocation in this example is also $\mathrm{Tb}$; the direction of the force is in the direction of motion of the dislocation, that is from front to back.

Figure 24 shows a screw dislocation as it glides all the way across the crystal. The symbol \$ denotes the dislocation. The slip vector and applied shear stress is the same as in figure 23, and the associated macroscopic strain is also the same; the crystal in the final state (d) is offset by a spacing $\underline{b}$, which is the same as in figure 23 (d). This result shows that the macroscopic striain associated with the glide of a dislocation through a crystal is a shear strain corresponding to the slip plane and slip direction of the dislocation. Thus the macroscopic strain is independent of the orientation of the dislocation. This point is often a source of confusion. When a dislocation sweeps out an area on its slip plane, the slip in the slipped area is uniquely determined by the slip vector. By the same general argument as for the edge dislocation above we can also show that the force on this screw dislocation is $\tau b$, but the direction is now from right to left.

In general, the force on a dislocation in glide is equal to the magnitude of the Burgers vector times the component of the applied stress in the slip plane and slip direction. The magnitude does not depend on the orientation of the dislocation in the slip plane. The direction of the force is at right angles to the dislocation, independent of the applied stress. A common error is to give the force the direction of the Burgers vector.

Figure 25 shows the climb of an edge dislocation. In the final state (d), a plane (the extra half plane that was above the dislocation) has been added to the crystal; the crystal has been increased in size by one interatomic spacing $b$ in the direction of the applied tensile stress. Again, as the dislocation moves and the crystal deforms, the applied stress $\sigma$ does work. When the dislocation moves all the way across the crystal, the work done is $\sigma b$ per unit area (the back face is moved by $b$ relative to the front face; the force on it is $\sigma$ per unit area). So the force on the dislocation is $\sigma b$ and its direction is down.

Here is a simple way to find the direction of the force on a dislocation from an applied stress on the crystal. If the dislocation were to move in the direction of the force on it, then the crystal would deform in such a way as to yield to the applied stress. This is clearly illustrated in figures 23,24 , and 25 .

Kinks and Jogs

In the above discussion of motion the dislocation was considered to be a straight line. However, if the motion takes place nonuniformly along the length of the dislocation, it results in kinks and jogs.

Figure 26 shows kinks in an edge and a screw dislocation. A kink is simply an offset in the dislocation line lying in the slip plane. It can be readily formed and annihilated by glide. Kinks arise because a dislocation generally prefers to lie along close packed directions in the crystal. A mixed dislocation making an angle with a close packed direction can be viewed as small segments lying along close packed directions, connected by kinks. The dislocation then has a zigzag form. The glide of the dislocation can be considered to occur by the motion of its kinks. As a kink 
moves along the length of the dislocation, the dislocation moves up by one Burgers vector. 7

Figure 27 shows jogs in an edge and a screw dislocation. A jog is an offset in the dislocation line that does not lie in the slip plane. For the edge dislocation in figure 27 (a) the jogs can only be eliminated by adding or removing vacancies or interstitial atoms at the edge of the extra half plane, $i . e$,, by nonconservative motion. The climb of an edge dislocation can be considered to occur by the motion of its jogs. As a jog moves along the length of the dislocation, the dislocation moves up by one Burgers vector. ${ }^{8}$

The term jog for a screw dislocation is not so clear, but is generally used. The jogs in figure 27 (b) could be eliminated by glide, but this glide must occur on another slip plane. 9 If the screw glides on its original slip plane, then the jogs can only move along by nonconservative motion. Jogs in screw dislocations may arise by a process known as cross glide, $i . e .$, the gliding screw dislocation crosses from one slip plane onto another.

Another way in which kinks and jogs may arise is by the intersection of dislocations. If one dislocation is intersected by another, the first dislocation acquires an offset equal in magnitude and direction to the Burgers vector of the second, and vice versa. This is easy to see in terms of the picture of slip. As the second dislocation moves, it extends the region of slip over the slip plane. Any line intersecting its slip plane, such as the first dislocation, will be offset by $b$ after passage of the second dislocation past the intersection point. The first dislocation will be kinked by an amount equal to the component of the offset parallel to its slip plane; it will be jogged by the component normal to its slip plane.

\section{Limit of Dislocation Velocity}

Finally let me advance another plausible reason for believing that slip occurs in a nonuniform way. A disturbance in a solid usually travels at a speed less than that of sound in the material. Therefore if stresses are applied to a crystal that make it deform plastically, the effect of the stresses on the surface will not propagate into the crystal any faster than the speed of sound. So at least for a sufficiently short time, one has to accept the idea that parts of the crystal have slipped and other parts have not. When the forces of deformation are withdrawn the crystal is left with many planes that have undergone partial slip; it is full of dislocations. The speed of sound is expected to be an upper limit on the dislocation velocity.

\section{Conclusion}

This has been an elementary paper. We have simplified certain concepts somewhat for the sake of clarity. There are many effects in the field of dislocations we have not discussed. To go into them would have led too far from the basic principles within the space allotted for this paper. It is hoped, however, that this paper has provided the foundation for understanding dislocation theory. We discussed how plastic deformation takes place through the motion of dislocation lines. The picture of slip has been emphasized, because it lends itself to a pictorial representation designed to give a physical feeling for dislocations. Another approach could have been used, emphasizing another aspect of dislocation theory. The basic ideas are illustrated by concrete examples which are physically possible, but somewhat simpler than actual crystals. The subsequent lectures of this symposium will undoubtedly introduce some of the more subtle aspects of dislocation theory.

${ }^{7}$ The kinks discussed here form abrupt right angles in the dislocation line as illustrated in figure 26. However, the line tension discussed earlier will tend to smooth these out somewhat.

${ }^{8}$ There is an analogy between the following two concepts: Slip proceeds by the motion of dislocations, and dislocation motion proceeds by the motion of kinks or jogs.

${ }^{9}$ In some crystals there are secondary slip planes which present more resistance to dislocation glide than the primary slip planes. If a screw dislocation has jogs on such a secondary slip plane they will not so readily be eliminated by glide. 
I am indebted to A. W. Ruff, Jr., for showing me the general method of constructing crystal models, and to $R$. Price for constructing the model of a bent dislocation shown in figure 11. L. M. Kushner kindly loaned me the slides from which figures 1 and 2 were made. I thank Gloria, my wife, for very helpful discussions and suggestions.

\section{Bibliography}

The following is a selected, but by no means complete, list of articles and books on the subject of dislocations. They have been chosen primarily for their introductory or review nature. The more elementary references are at the beginning and the more difficult ones at the end of the list.

1. N. F. Mott, Atomic Structure and the Strength of Metals, Pergamon Press, 62 pp, (Oxford, 1956 ).

Subtitled "An account for the nonscientist of recent researches aimed at understanding why metals have their characteristic strength and ductility, "this is a very elementary and lucid account of dislocation theory and some of its consequences. The book consists of two lectures prepared in response to an invitation to give the Page-Barbour lectures before the University of Virginia in October 1956.

2. A. G. Quarre11, Dislocations in metals, The Structure of Metals, A Modern Conception, Ch. 3, pp. 59-83, Iliffe \& Sons (London, 1959).

This is one of four lectures delivered for the Institution of Metallurgists' Refresher Course, held at Estbourne in Autumn 1958. It is a brief and simple discussion on some of the major problems of dislocation theory and their solution. The article starts with considerations as to why it has been necessary to introduce the concept of a dislocation. It goes on with giving simple pictures of the various types of dislocations. Then it discusses the origin, multiplication and stress to move dislocations, followed by an explanation of partial and sessile dislocations. The article concludes with applications, where some of the major successes of dislocation theory are outlined. This lecture may well be the quickest introduction to the subject of dislocation theory.

3. J. M. Burgers and W. G. Burgers, Dislocations in crystal lattices, Rheology $\underline{1}$, 141-199 (1956).

This is an excellent introductory and review article, written in a simple and readable style. It starts out with a general discussion on dislocations in relation to a continuous medium and an atomic lattice. The largest part of the paper is concerned with dislocations in particular lattices: the simple cubic, the face-centered and body-centered cubic, other lattices consisting of single species of atoms, and lattices of more than one atomic species, such as rock salt and calcite. Finally there is discussion of the interactions between dislocations. The authors have taken great pains throughout the article to document their statements with detailed references; often they go so far as to indicate the exact pages in the references to consult. This makes the paper a valuable reference source.

4. J. S. Koehler and F. Seitz, The nature of dislocations in ideal single crystals, Dislocations in Metals, ed. M. Cohen, A.I.M.E., pp. 1-36, (New York, 1954).

This is one of three articles that grew out of survey lectures given at a seminar held by the Institute of Metals Division, A.I.M.E., in October 1951. The presentations were educational in nature, and purposely not intended to take the form of original papers; so the result is somewhat reminiscent of the present symposium. The article by Koehler and Seitz is a simple account of the "need" for dislocations and an outline of the various phenomena in which they come into play. They review the geometry, stress-fields, energetics, and interactions of dislocations.

5. W. T. Read, Jr., and W. Shockley, On the geometry of dislocations, Imperfections in Nearly Perfect Crystals, ed. W. Shockley, John Wiley \& Sons, pp. 77-94, (New York, 1952).

This paper contains a discussion of dislocations in terms of the Burgers circuit; the Burgers vector as well as the concepts of "good" and "bad" regions of the crystal are treated in relation to the Burgers circuit. The article further discusses the motion of dislocations, partial dislocations, slip bands, the intersection of dislocations, and the Frank-Read mechanism of dislocation multiplication. 
6. W. T. Read, Jr., Dislocations in Crystals, McGraw-Hill Book Co., 228 pp., (New York, 1953).

This is a good introductory textbook, and the principal reference for this lecture. The background it assumes on the part of the reader is elementary: a little of crystallography, elasticity, calculus, and general physics. The book uses many pictures and Read has taken great pains to offer a clear introduction to fundamental concepts. The first and most useful part of the book deals with the basic ideas of dislocation theory. This includes the geometrical, crystallographic, and analytical properties of dislocations, and certain special but important subjects such as multiplication and intersection of dislocations. The second part of the book deals with the application of theory to crystal growth and grain boundaries.

7. C. Kittel, Dislocations, Introduction to Solid State Physics, John Wiley \& Sons, Ch. 19, pp. 536-570, New York, 2d ed. (1956).

This is the last chapter of Kittel's book, well known to solid state physicists. It can be read, however, without reference to the rest of the book, and forms by itself a good review article of the more important aspects of dislocation theory.

8. F. Seitz, The theory of plastic flow in single crystals, plastic Deformation of Crystalline Solids, Carnegie Institute of Technology, pp. 1-36, (1950).

This is a fundamental review article, which is brief and clear while still fair1y comprehensive. It was written before direct experimental evidence for dislocations had been obtained. The first part of the paper describes some of the plastic properties of single crystals that dislocations are purported to explain. The middle part gives the fundamental properties of a dislocation. Seitz's description of a dislocation forms the basis for the general definition of a dislocation we have used in the present paper. The last part of the article goes into applications of dislocation theory, some of which have been modified in the light of newer knowledge.

9. N. F. Mott, Dislocations in crystalline solids, Surveys in Mechanics, pp. 32-63, (Cambridge, 1956).

This is one of "a collection of surveys of present position of research in some branches of mechanics, written in commemoration of the 70 th birthday of $G$. I. Taylor." After a brief introduction to the nature of dislocations, the article covers a range of practical problems where dislocation theory has been applied. These include such subjects as the energy of grain boundaries, the origin of slip lines, the stress to move a dislocation, and problems related to diffusion.

10. A. H. Cottre11, Dislocations and Plastic Flow in Crysta1s, Oxford, 223 pp. (1953). This is another good introductory text to dislocations and their role in plastic deformation, but more condensed and on a more sophisticated level than Read's book above. Together these two books can be regarded as the standard references in the field, notwithstanding the early date of publication. Cottrell covers the quantitative development of dislocation theory in a thorough way in his first three chapters; he then applies it to a number of practical problems in plastic deformation such as yield strength, work-hardening, annealing, and creep in the last two chapters.

11. J. J. Gilman, Mechanical behavior of ionic crystals, Progr. Ceram. Sci. 1, 146199 , (1961).

This is a review of the rock-salt type crystal structure with special emphasis on the by now classic work of J. J. Gilman and W. G. Johnston on LiF. The article begins with a discussion of the theory of cohesion of ionic crystals to justify the theoretical model of an ionic crystal. This is followed by a discussion of the elastic behavior. Then an extended discussion is given of "microplasticity," i.e., the behavior of individual dislocations. This includes the geometry, energy, techniques for observation, measurements of motion, origin and multiplication of dislocations. Next, the collective behavior of large numbers of dislocations is considered in "macro-plasticity." This touches on stressstrain curves, creep, and internal friction. Finally, the problem of fracture is considered, and the ways in which cracks are nucleated and propagated are discussed.

Two useful references to consult for up-to-date review articles are the following series:

1. Solid State Physics, ed. F. Seitz and D. Turnbul1, Academic Press, New York.

2. Progress in Materials Science, ed. B. Chalmers, Pergamon Press, New York. 

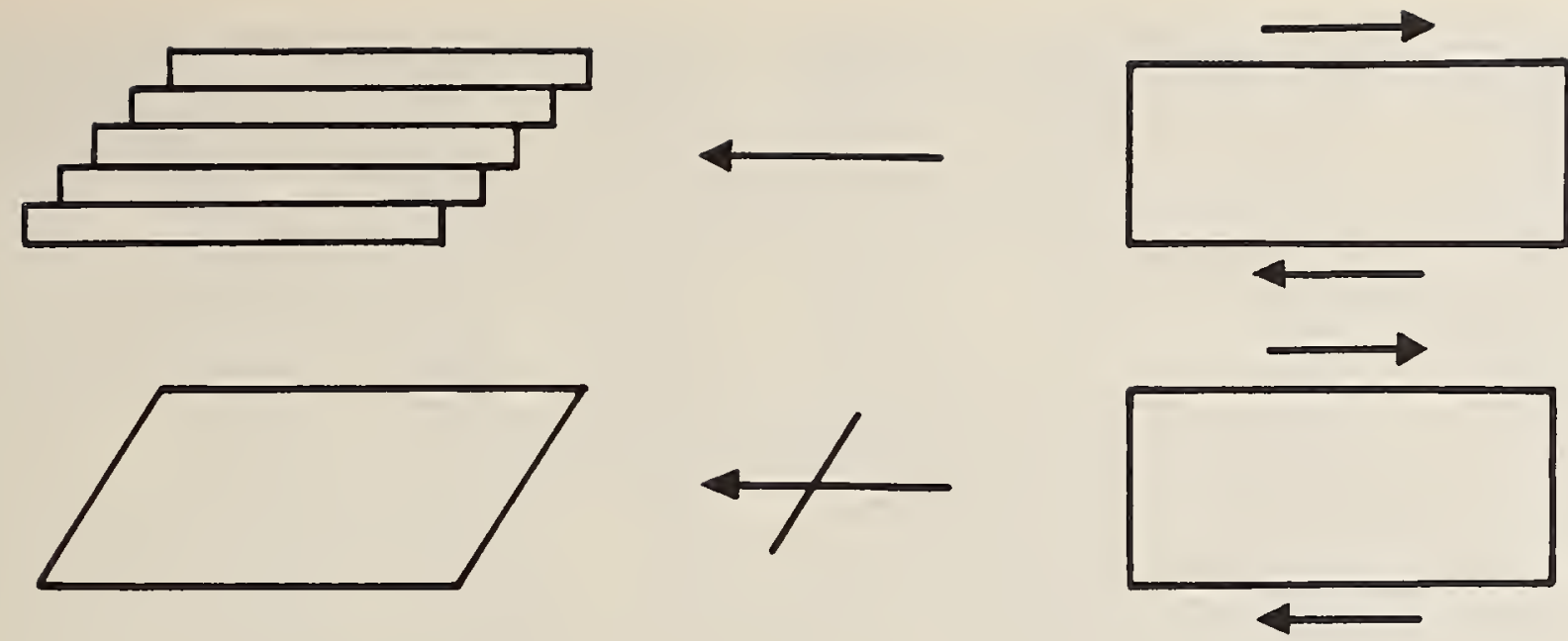

FIGURE 1. Plastic deformation of a crystalline solid does not take place by a uniform shear, as shown at the top; rather, it takes place by planes sliding over one another along well-defined crystallographic directions, as shown at the bottom.
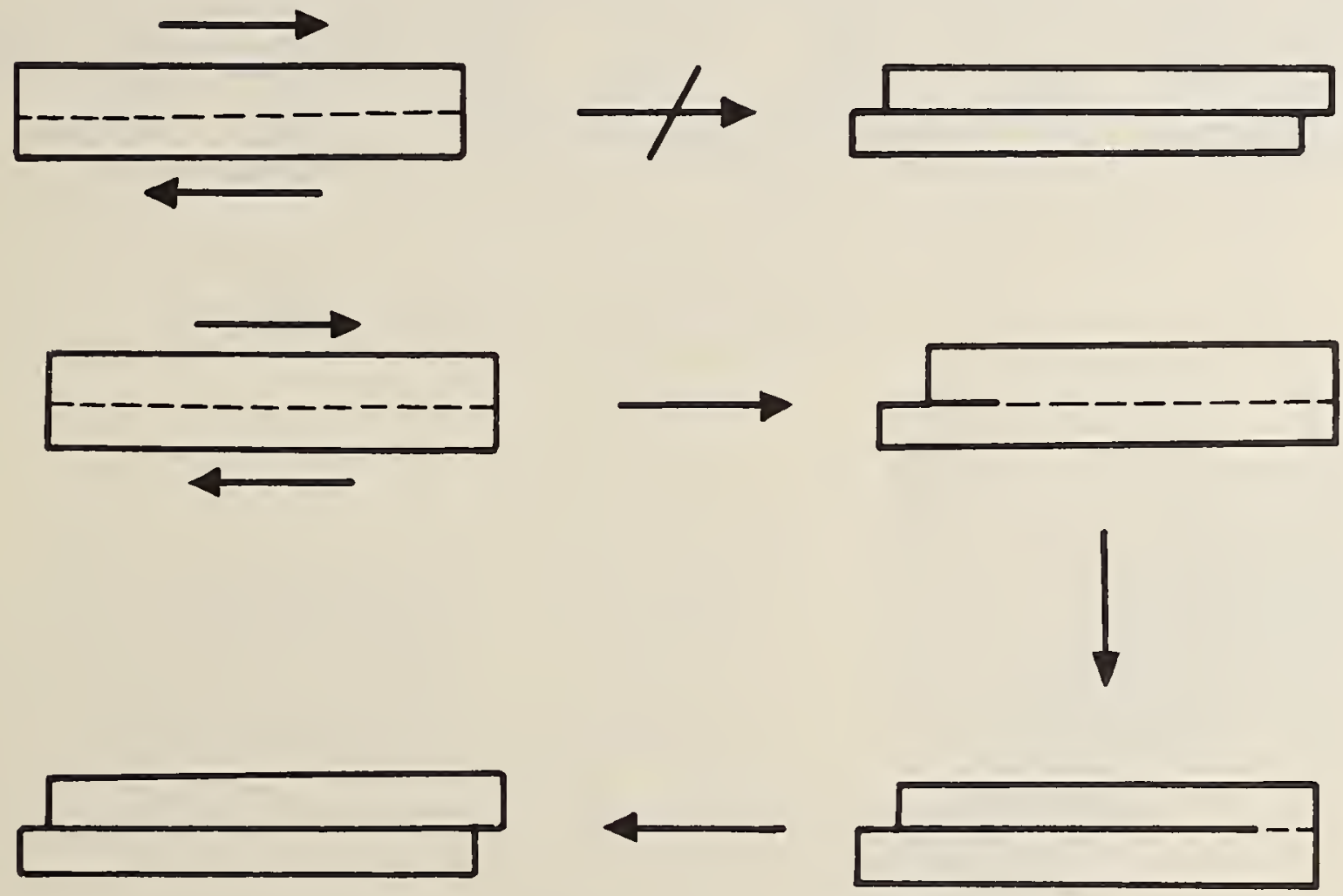

FIGURE 2. Plastic deformation by slip is not simultaneous and uniform over the whole slip plane, as shown at the top; rather, it is a local process and it proceeds by the gradual extension of regions of local slip, as shown at the bottom. The boundary between the slipped and unslipped regions is a dislocation. 


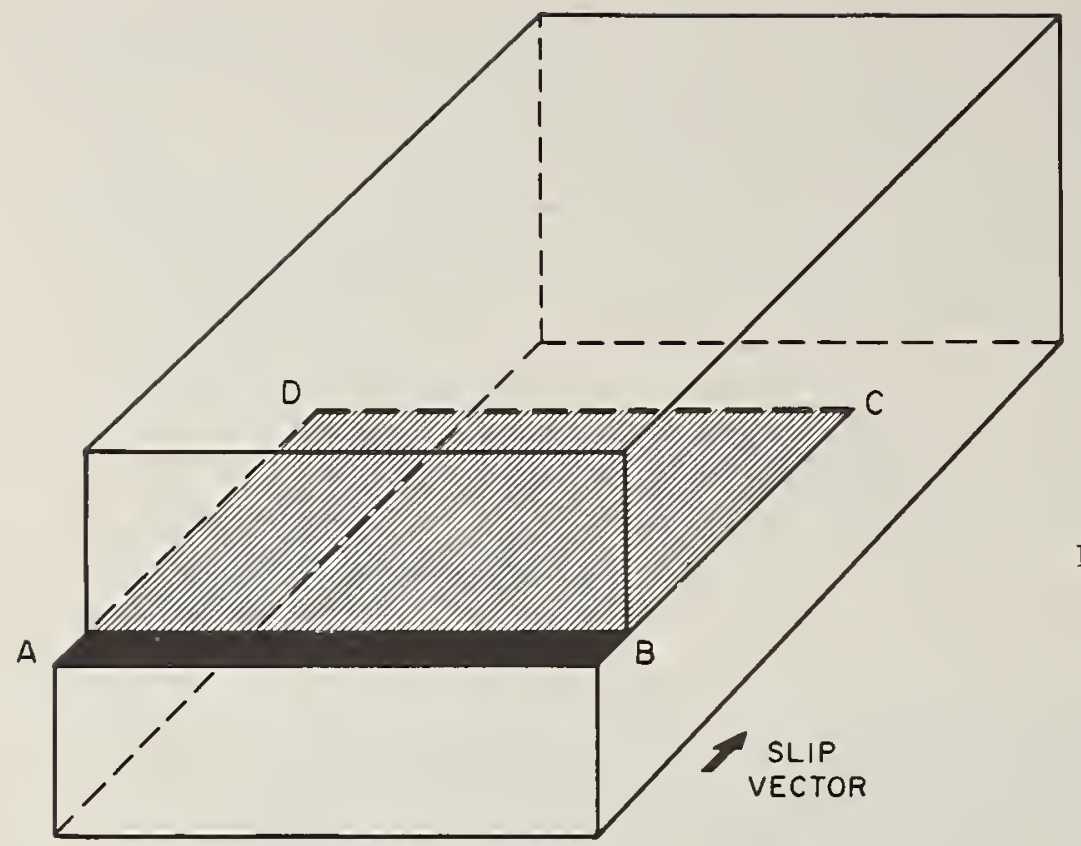

FIGURE 3. The slip that produces an edge dislocation. Unit slip has occurred over the area $A B C D$. The boundary $C D$ of the slipped area is the dislocation; it is perpendicular to the slip vector.

FIGURE 4. Arrangement of atoms around the edge dislocation shown in figure 3 . The plane of the figure is parallel to the slip plane. $A B C D$ is the slipped area and $C D$ the dislocation. The open circles represent atoms in the atomic plane just above the slip plane and the solid circles, atoms in the plane just below the slip plane.

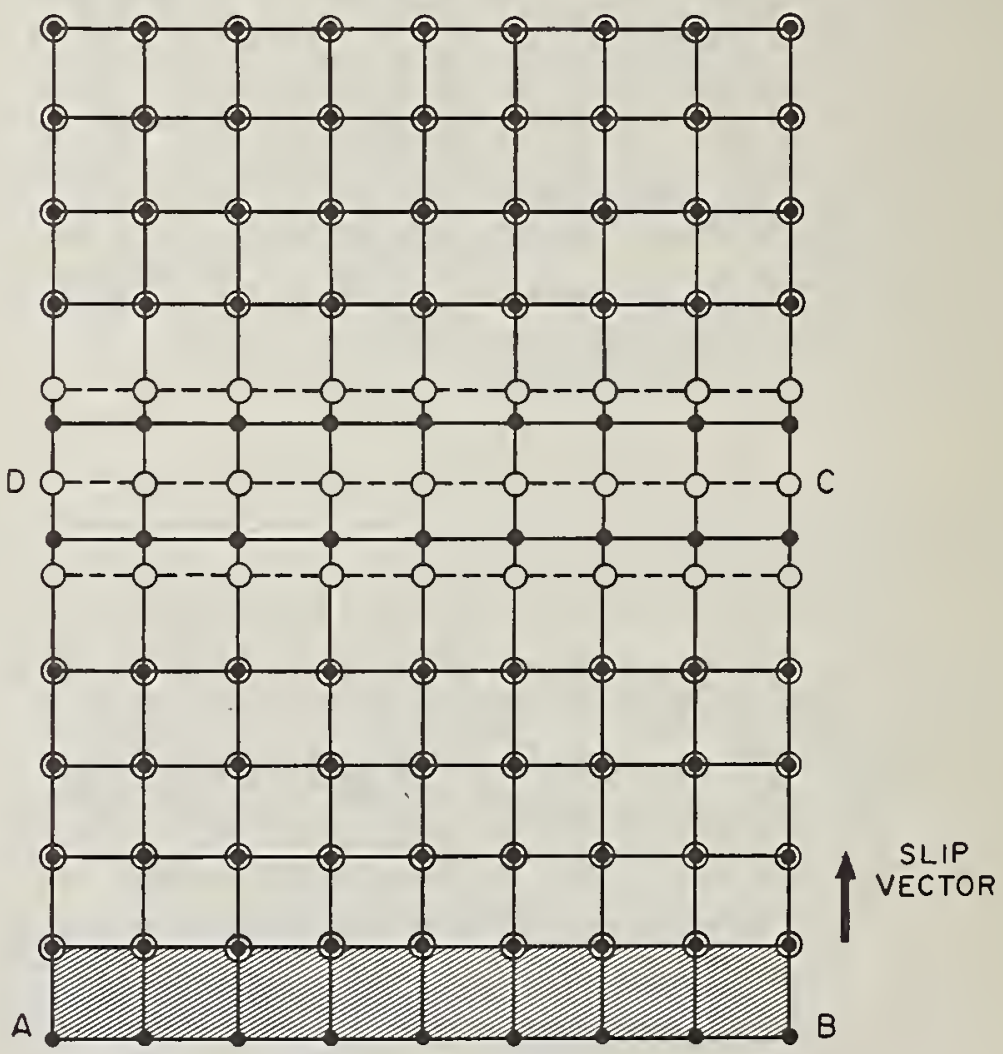




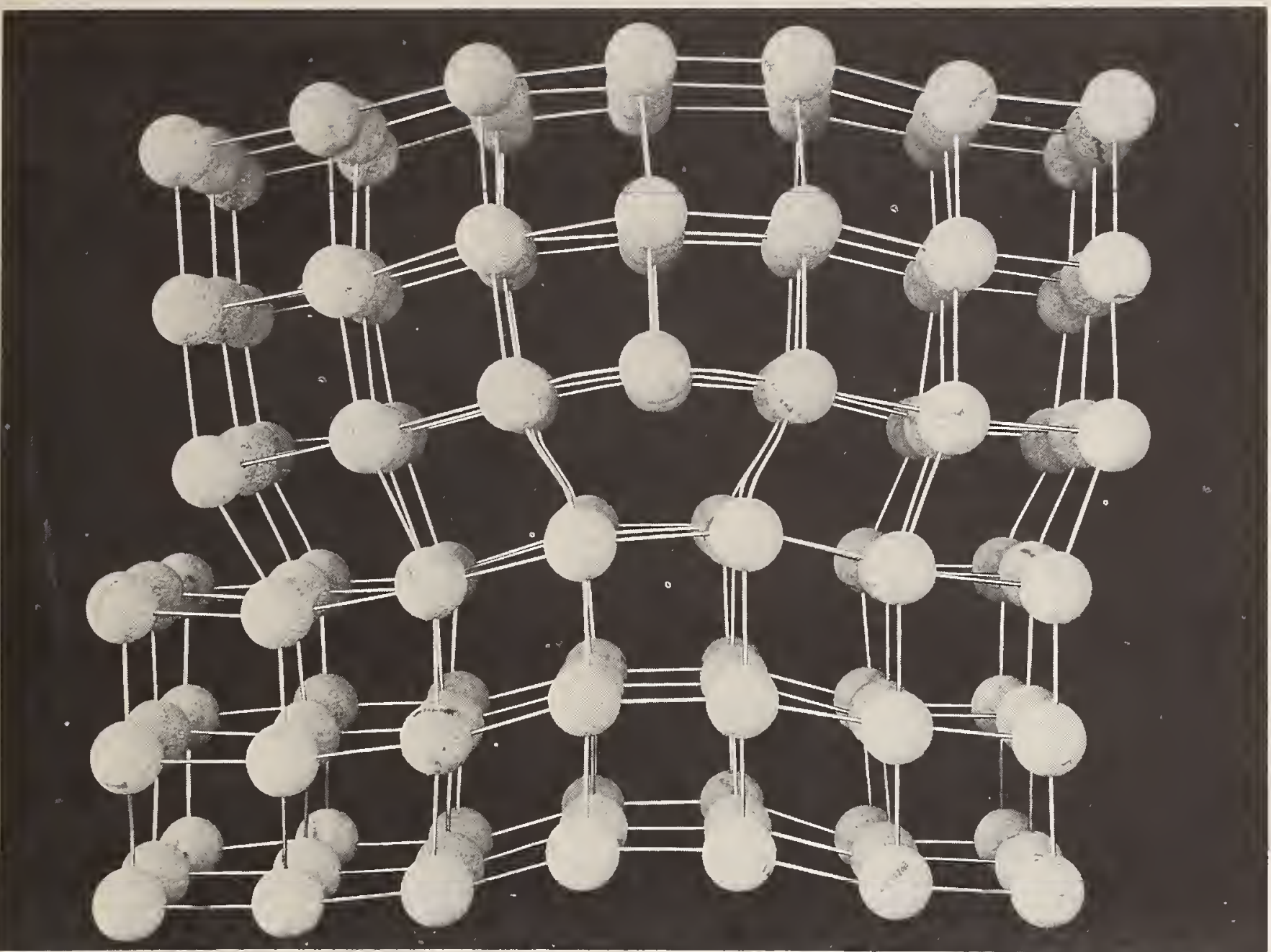

FIGURE 5. Photograph of a crystal model of an edge dislocation. The crystal is oriented so that it is viewed parallel to the dislocation line (CD in figures 3 and 4). The dislocation is a region of severe atomic misfit, where atoms are not properly surrounded by their neighbors. Note that the dislocation is the edge of an incomplete atomic plane; hence, the name edge dislocation.

FIGURE 6. The slip that produces a screw dislocation. Unit slip has occurred over ABCD. The screw dislocation $A D$ is parallel to the slip vector. (From Dislocations in Crystals by W. T. Read, Jr. copyright 1953 , McGraw-Hil1 Book Co., Inc. Used by permission.)

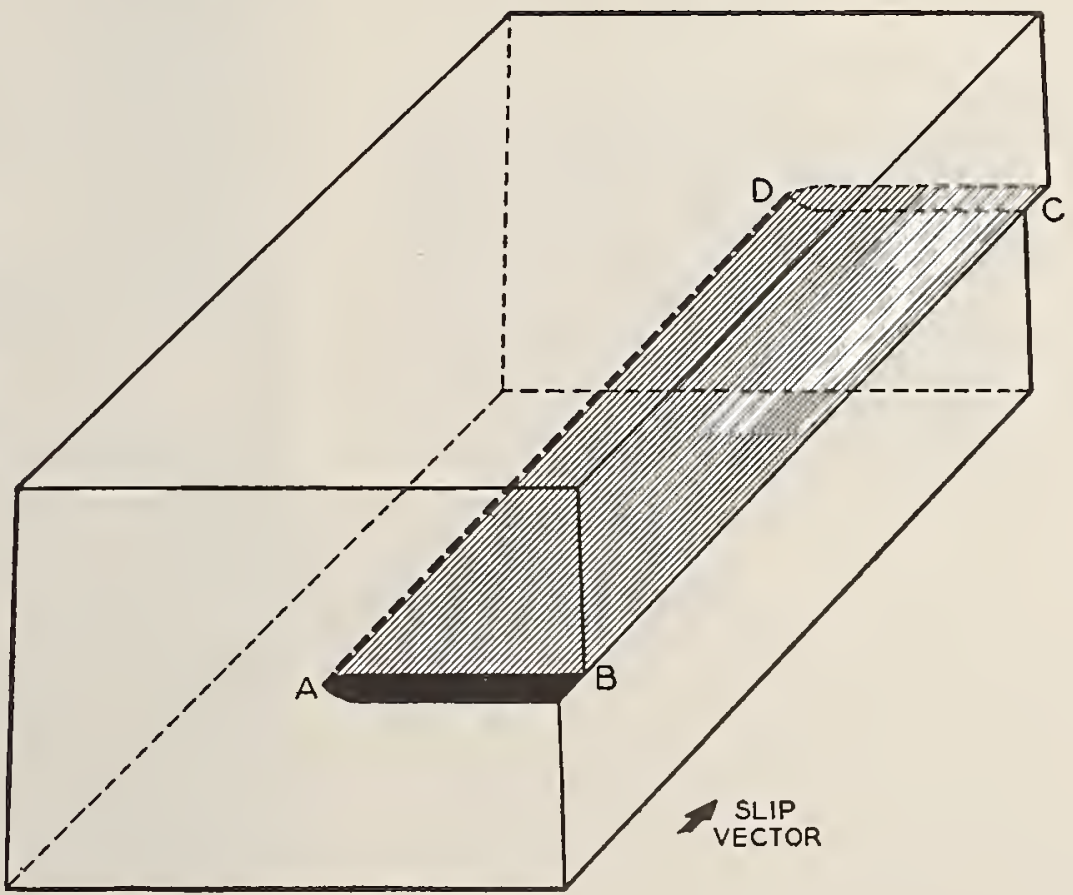




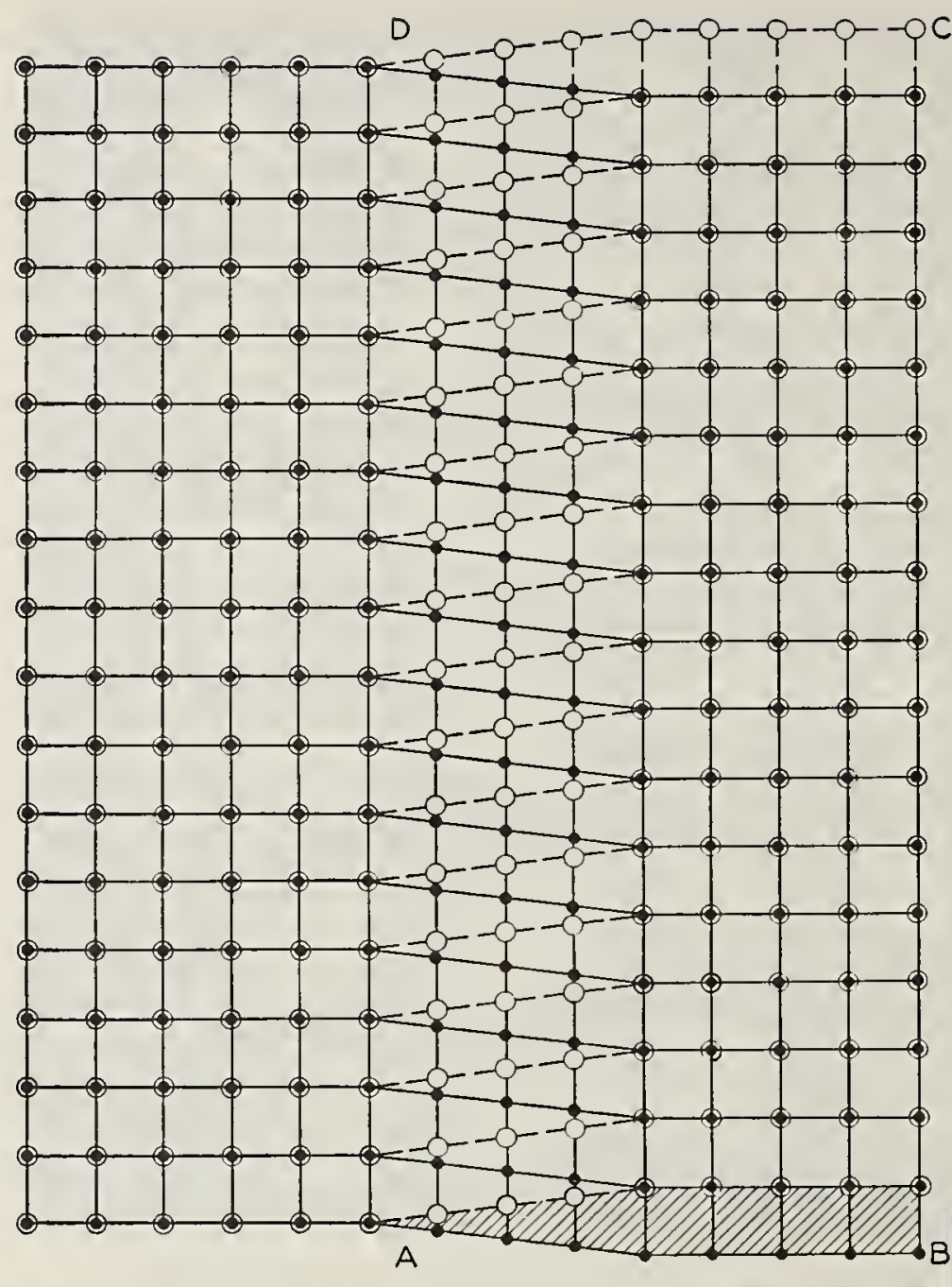

FIGURE 7. Arrangement of atoms around the screw dislocation shown in figure 6. Open circles represent the atomic plane just above the slip plane; solid circles represent the atomic plane just below. (From Dislocations in Crystals by W. T. Read, Jr., copyright 1953, McGraw-Hill Book Co., Inc. Used by permission.)

FIGURE 8. Crystal model of a screw dislocation. The model is viewed perpendicular to the dislocation line (or parallel to $C D$ in figures 6 and 7). The dislocation runs horizontally through the center of the crystal, paraliel to the slip vector. Note that the crystal is a single atomic plane in the form of a helicoid, or spiral ramp; hence, the name screw dislocation. 


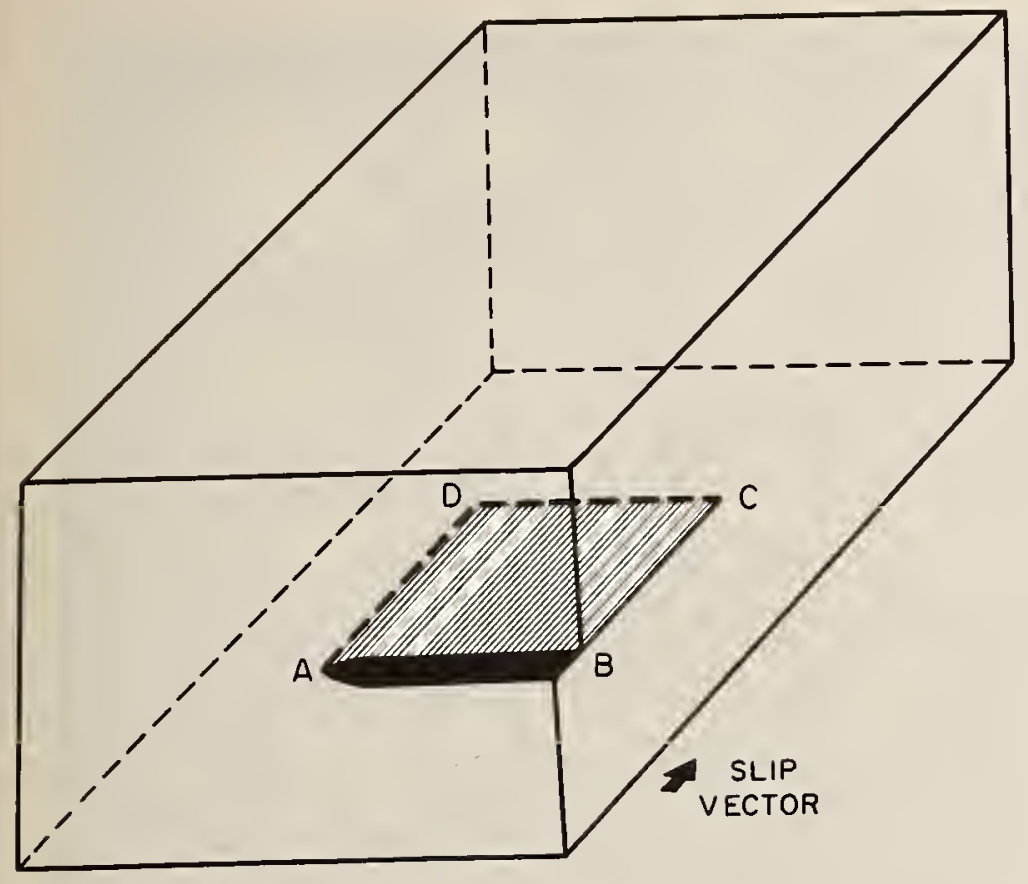

FIGURE 9. Dislocation with right angle bend. Unit slip has occurred over the area $A B C D$. The dislocation line is CDA; $C D$ is in edge and $A D$ in screw orientation. (Adapted from Dislocations in Crystals by $W$. T.

Read, Jr., copyright 1953, McGraw-Hill Book Co., Inc. Used by permission.)

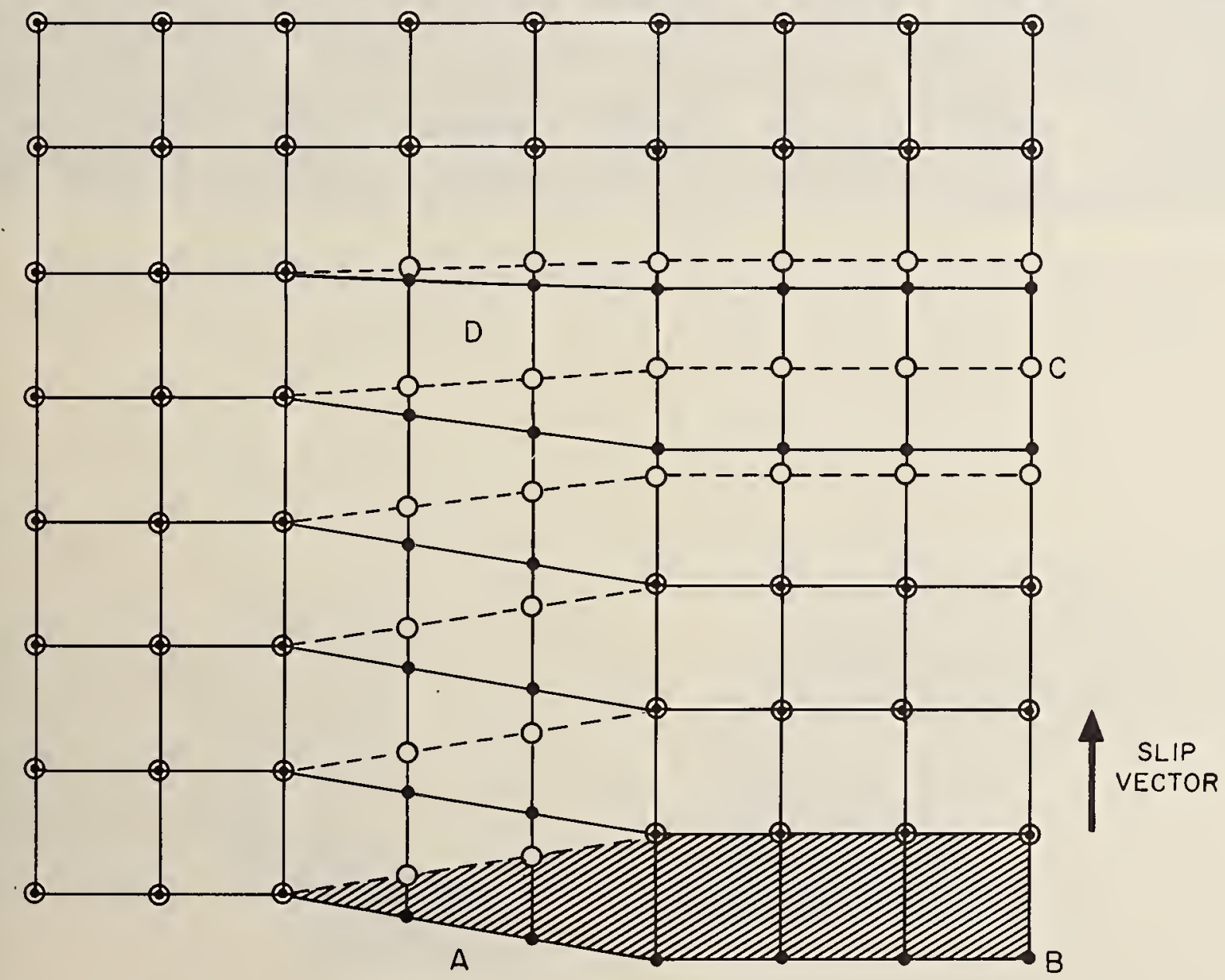

FIGURE 10. Arrangement of atoms for the dislocation shown in figure 9. The open circles represent atomic planes above the slip plane $A B C D$, the solid circles, those below. 


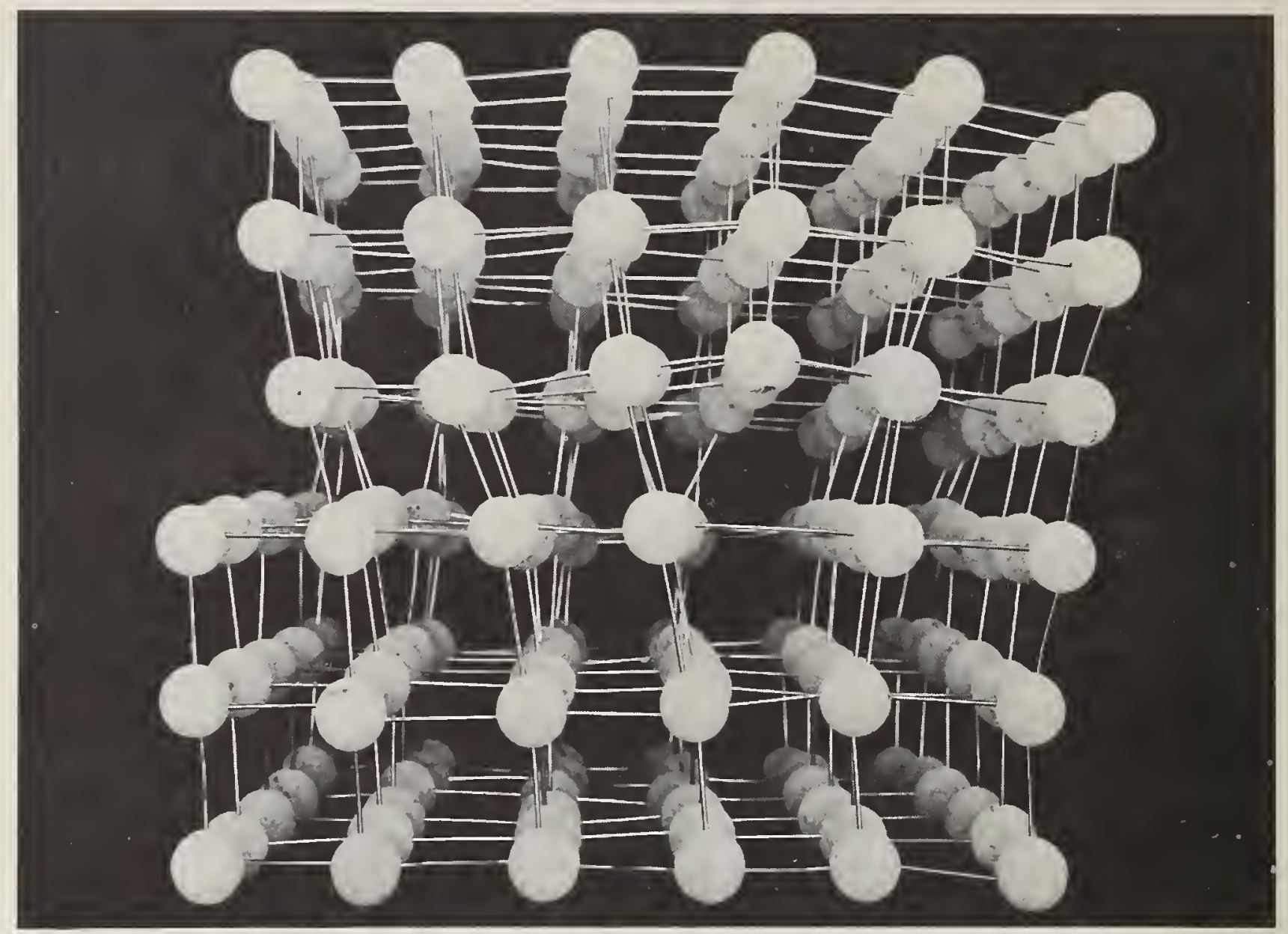

FIGURE 11. Model of dislocation with right angle bend. The model is viewed parallei to the edge and perpendicular to the screw component of the dislocation (along the line $C D$ in figures 9 and 10). Compare this figure with figures 5 and 8 .

FIGURE 12. Unit slip in the area $A B C$ produces a curved dislocation $A C$ lying in a single slip plane. (From Dislocation in Crystals by W. T. Read, Jr., copyright 1953, McGraw-Hili Book Co., Inc. Used by permission.)

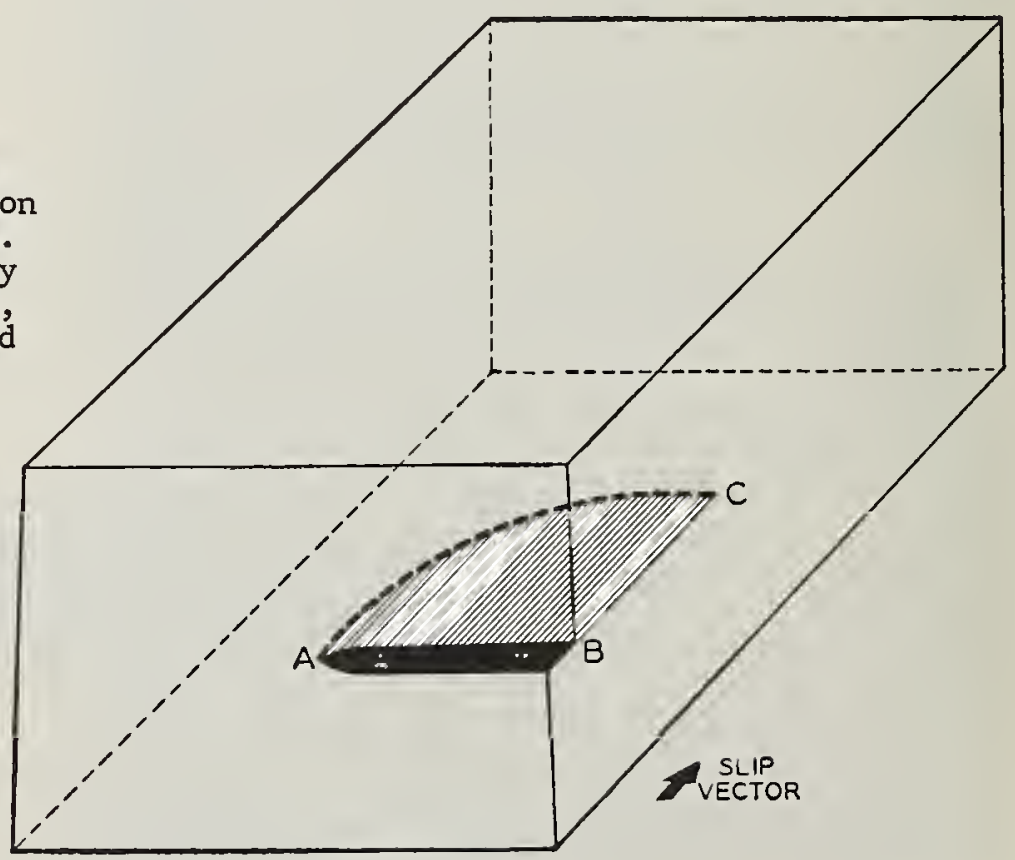




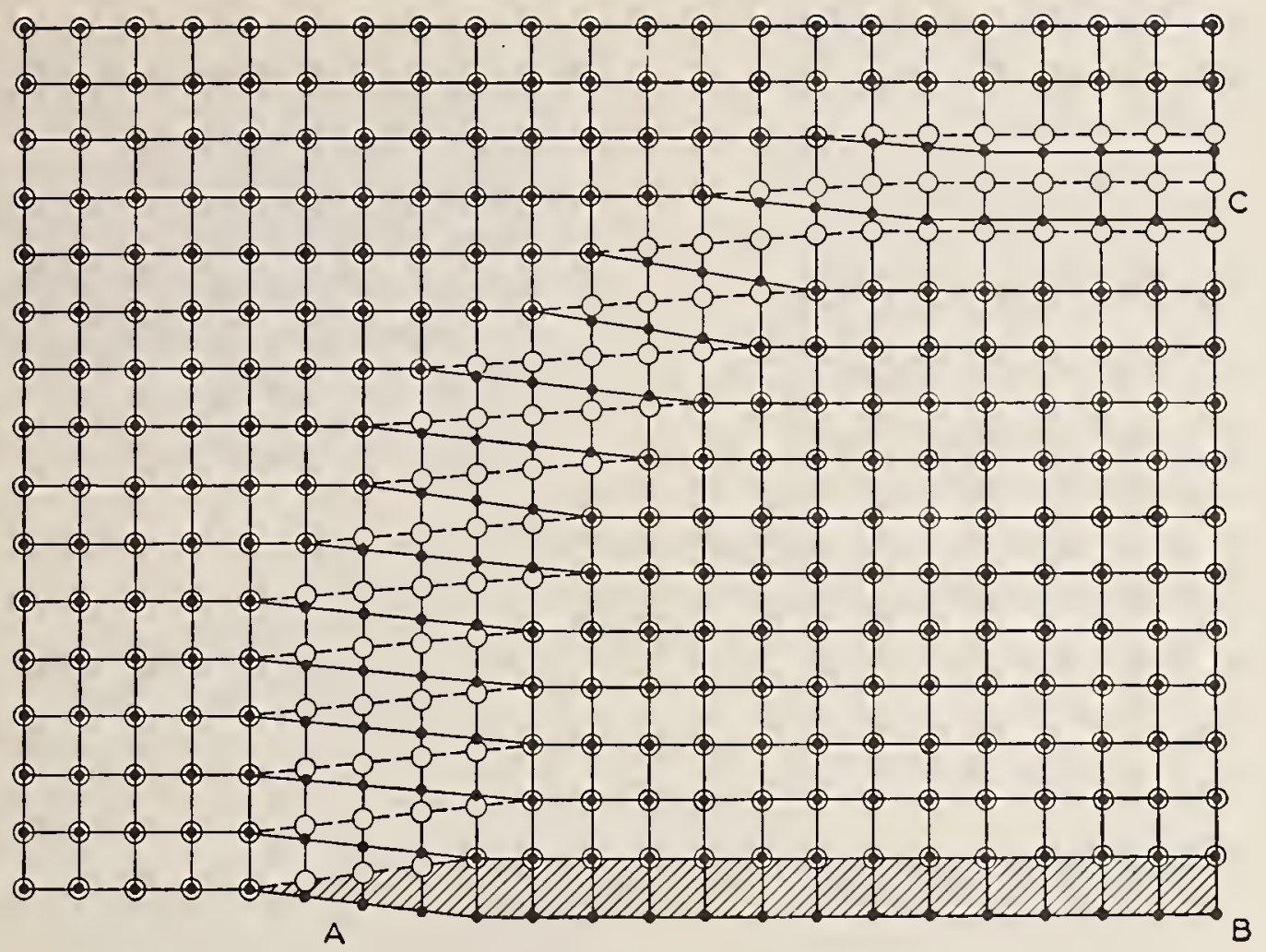

FIGURE 13. Arrangement of atoms for the curved dislocation shown in figure 12. Open and solid circles represent atoms just above and just below the slip plane. (From Dislocations in Crystals by $\mathrm{W}$. T. Read, Jr., copyright 1953, McGraw-Hill Book Co., Inc. Used by permission.)

FIGURE 14. An example of slip that produces a bent dislocation which does not lie in a single slip plane. The boundary $\mathrm{CDE}$ of the slipped area is an edge dislocation. (From Dislocation in Crystals by W. T. Read, Jr., copyright 1953, McGraw-Hill Book Co., Inc. Used by permission.)

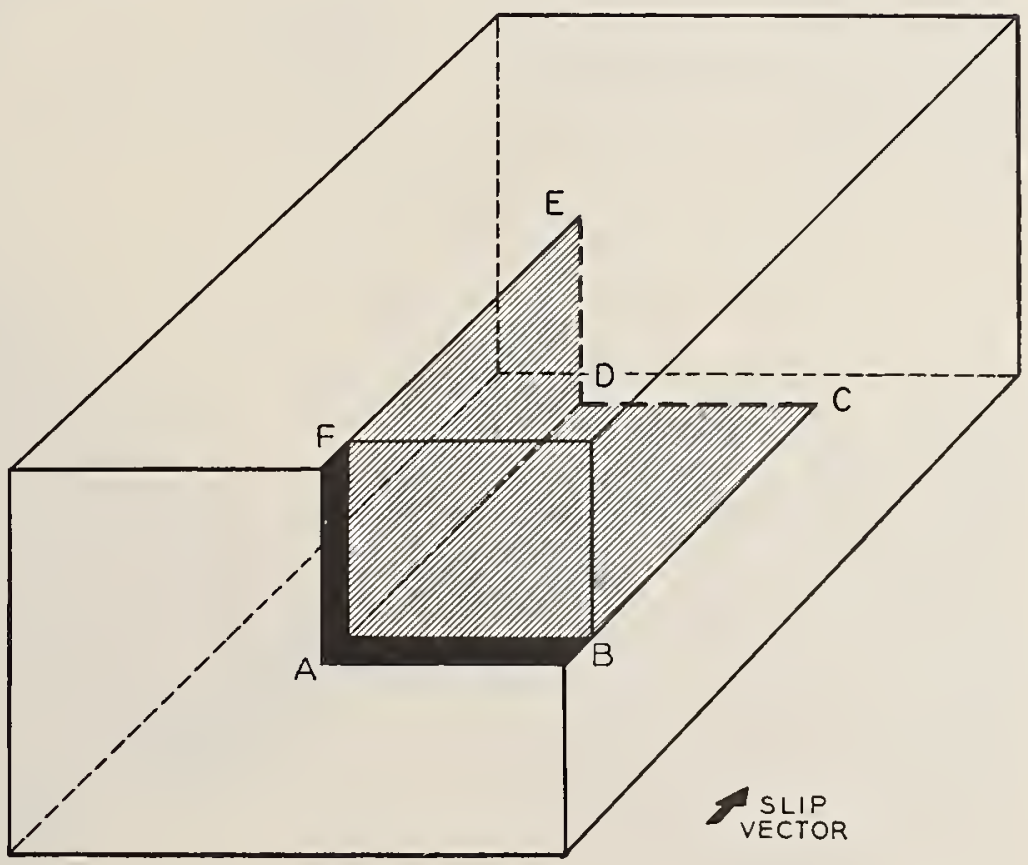




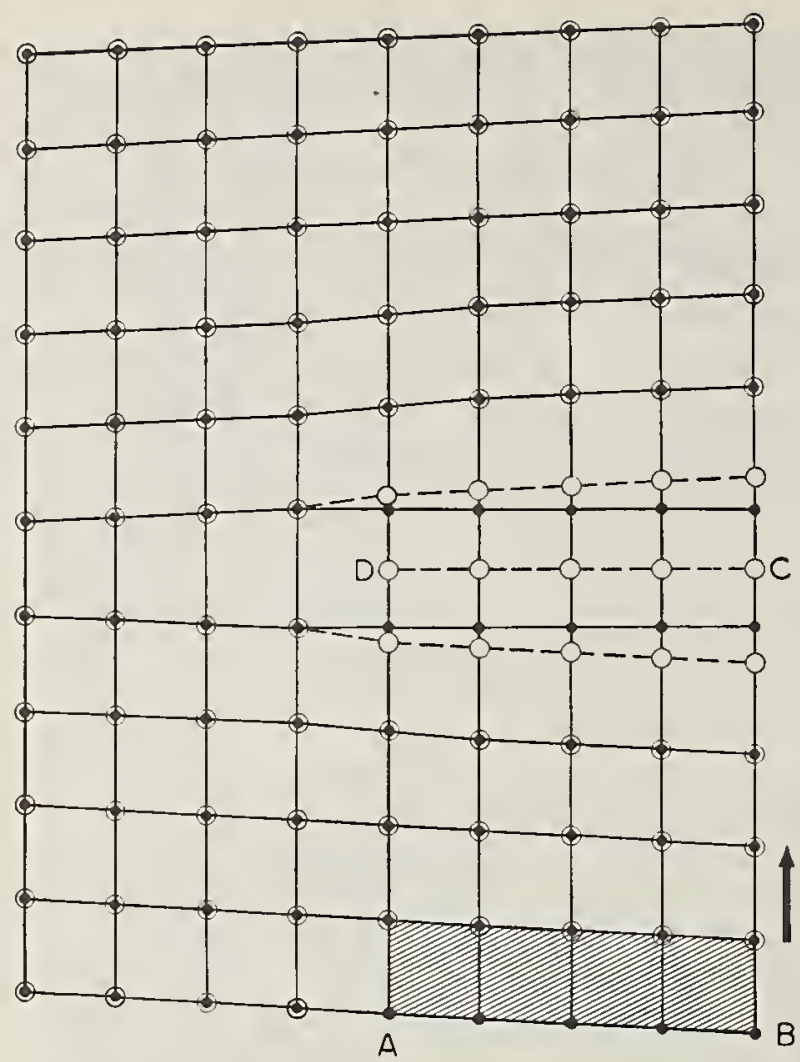

FIGURE 15. Arrangement of atoms in a plane parallel to $A B C D$ of figure 14. The section of dislocation CD lies in the plane of the drawing; the section $\mathrm{DE}$ is normal to the drawing. (From Dislocations in Crystals by $W$. T. Read, Jr。, copyright 1953, McGraw-Hil1 Book Co., Inc. Used by permission.)

FIGURE 16. A general slip surface. The surface contains the slip vector everywhere, but otherwise has an arbitrary shape. Unit slip has occurred over the area ABCD. The curve $C D$ is everywhere perpendicular to the slip vector, and hence an edge dislocation.

FIGURE 17. A general dislocation. Slip on the same general slip surface as shown in figure 16, has proceeded by different amounts on different parts of the surface. The curve $C D$ is a dislocation of continuous $1 y$ varying orientation. A dislocation following a completely arbitrary curve in the crystal can be formed by the appropriate choice of the slip surface and the extent of slip on it.

A
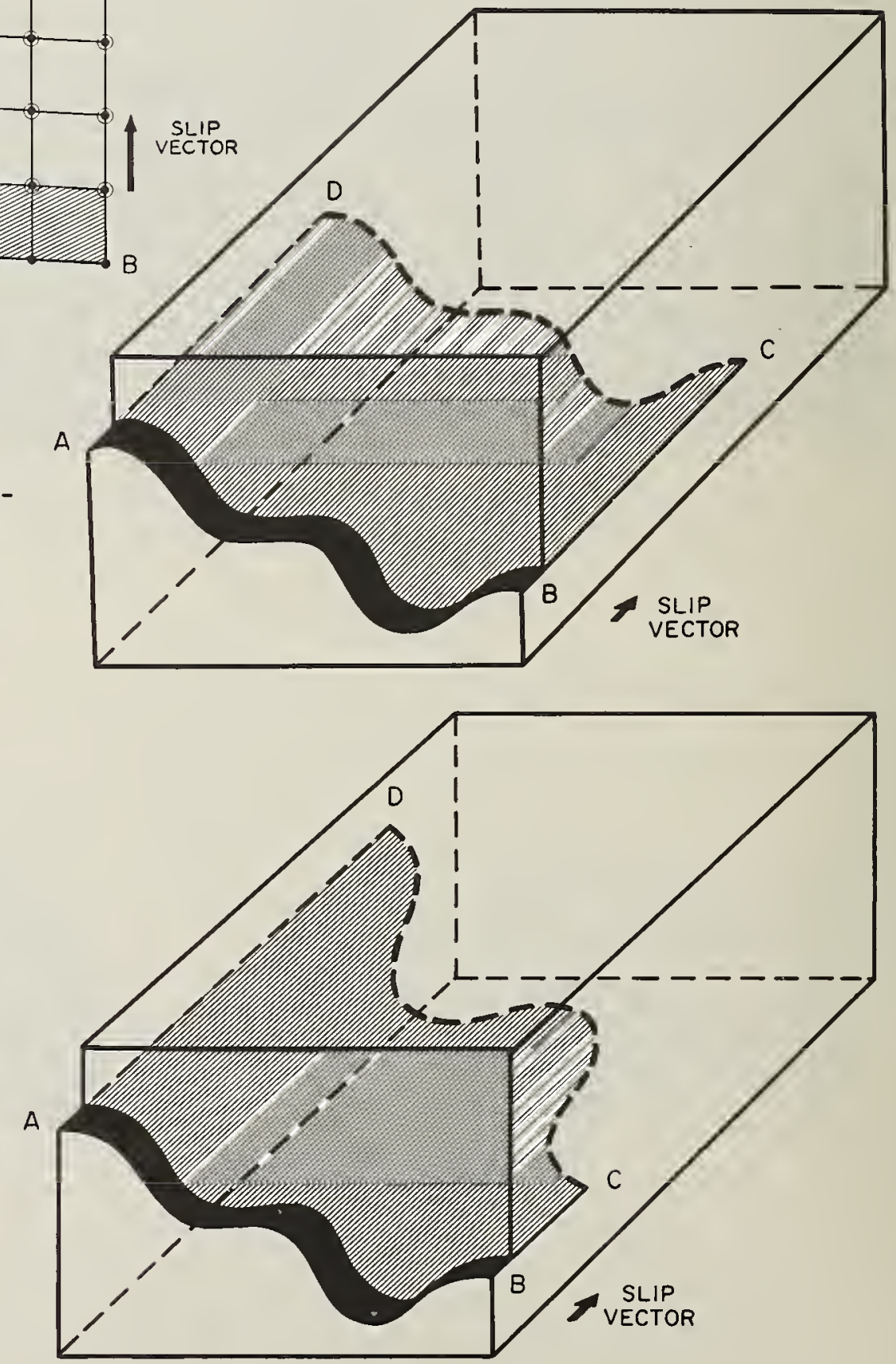
FIGURE 18. Construction of a general dislocation. The surface $\mathrm{S}$, bounded by the closed curve $C$, is shown with the sign convention for the normal $n$ to $S$. A cut is made along $S$, and the negative side of the cut is displaced by b relative to the positive side. The result is a general dislocation along $C$.
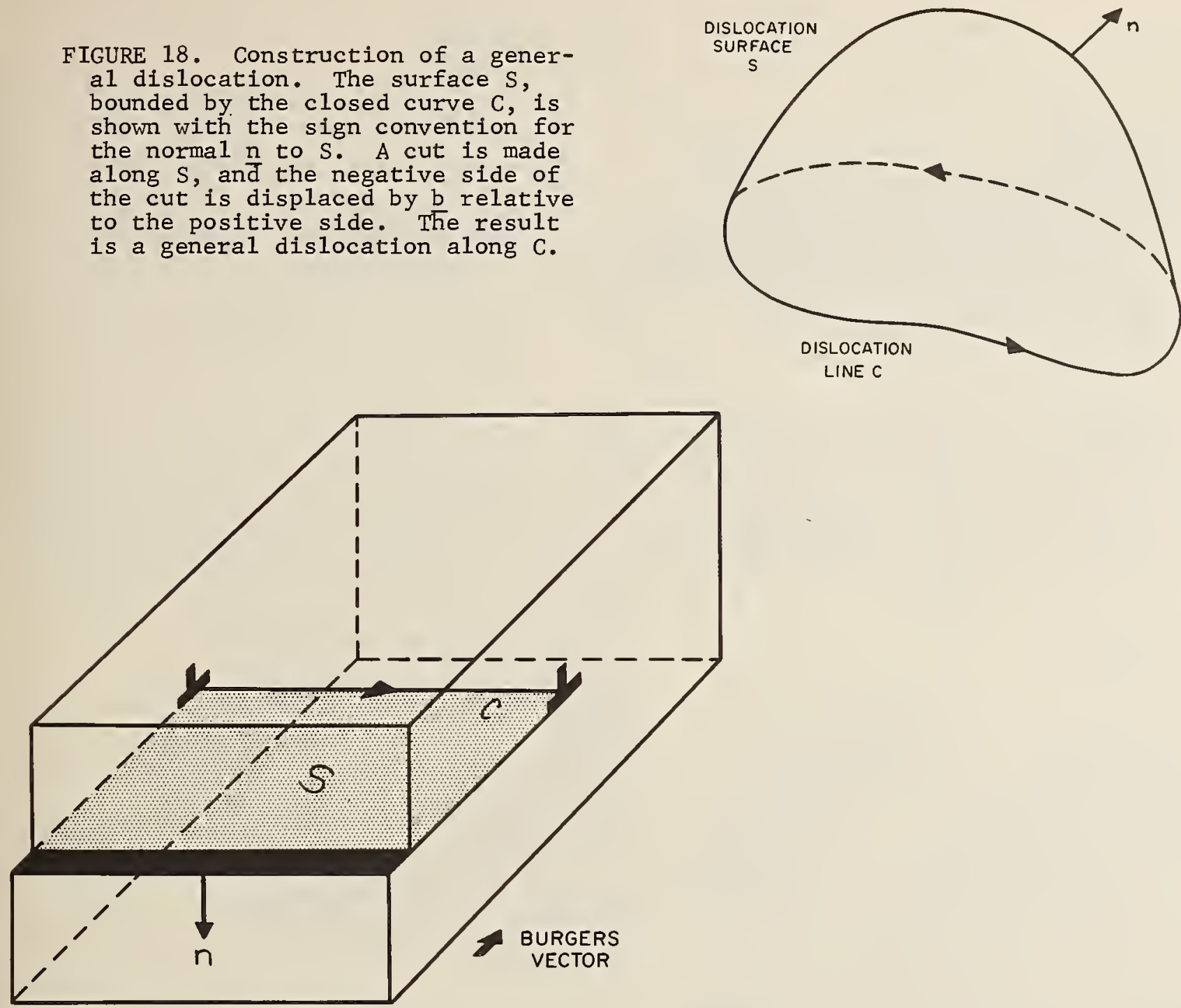

(a)

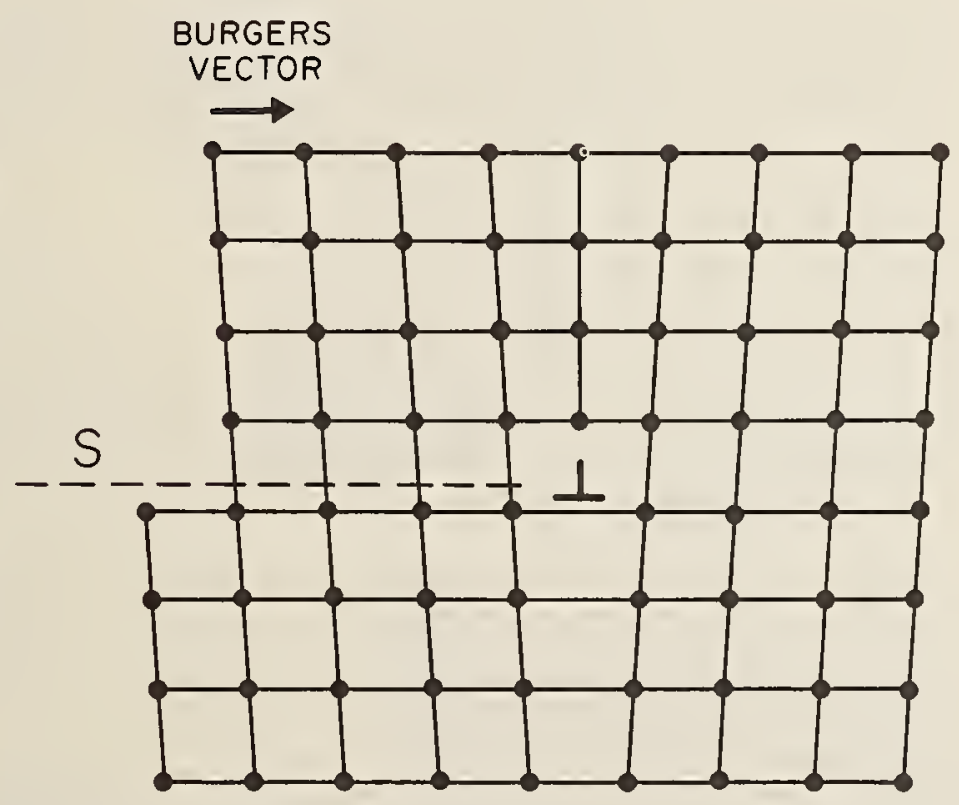

FIGURE 19. Construction of an edge dislocation. The surface $S$ is the slipped region of the slip plane. The symbol 1 denotes the dislocation. (a) Three dimensional view (similar to figure 3 ); the symbols defined in figure 18 are shown for this particular case. (b) A plane of atoms normal to the dislocation line (similar to figure 5). Note that the crystal is in register across the surface S. (Part (b) is adapted from Dislocations in Crystals by $w_{.} T_{0}$ Read, Jr., copyright 1953, McGraw-Hili Book Co., Inc. Used by permission.)

(b) 

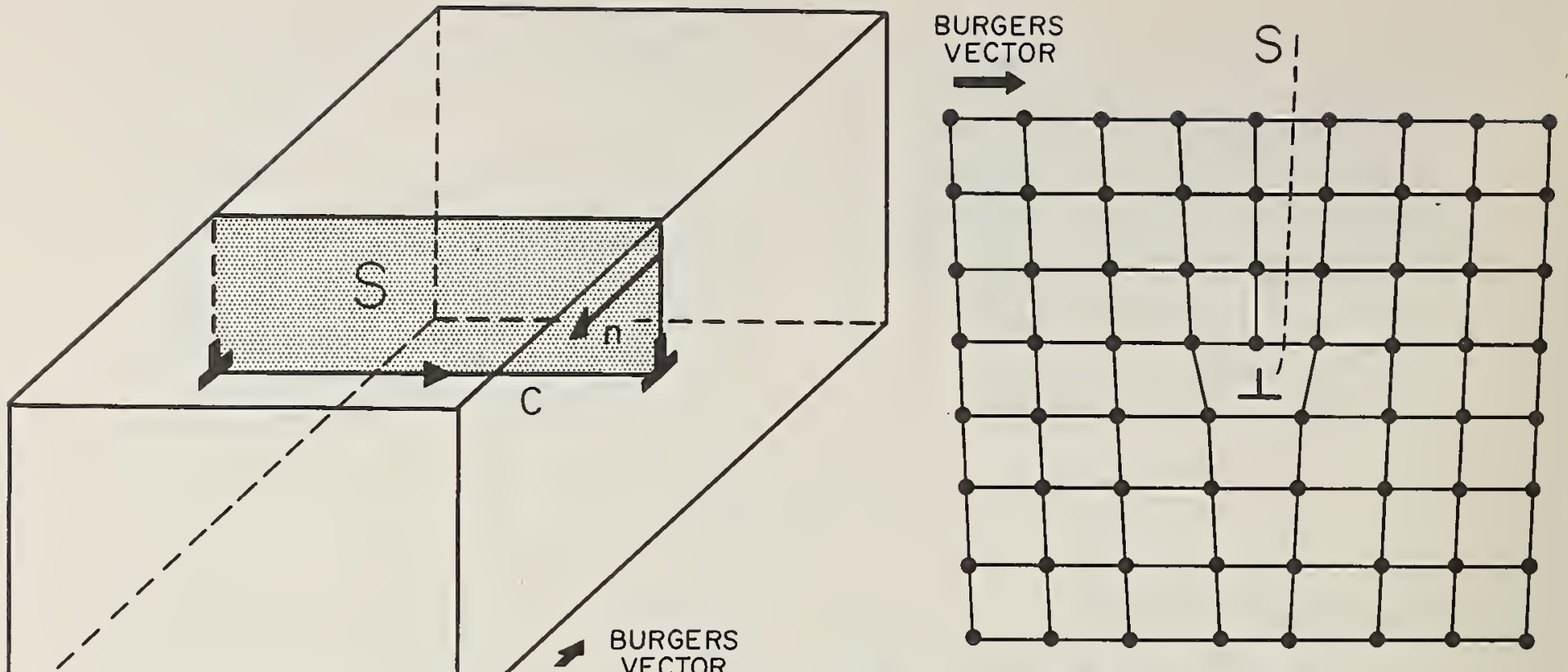

VECTOR

(a)

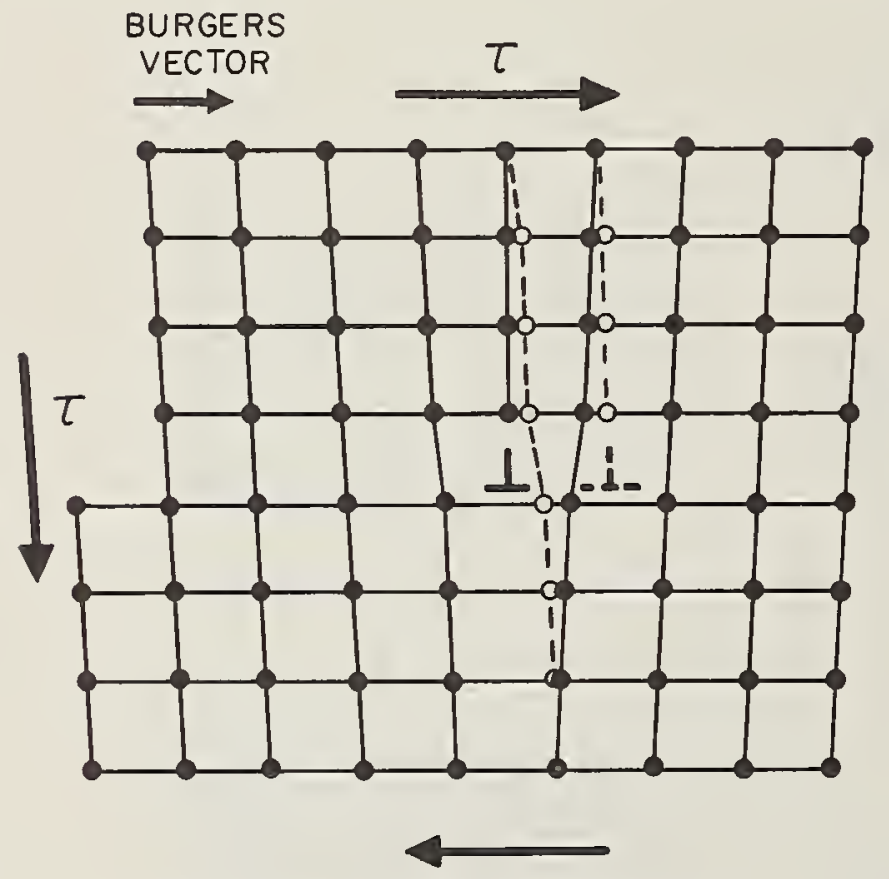

FIGURE 20. Another construction of an edge dislocation. The surface $S$ is the extra half plane above the dislocation. (a) Three dimensional view showing the symbols defined in figure 18. (b) A plane norma1 to the dislocation. The atoms are in register across S. Note that the resulting dislocation is identical to the one in figure 19 (b). (Part (b) is adapted from Dislocations in Crystals by W. T. Read, Jr., copyright 1953, McGraw-Hi11 Book Co., Inc. Used by permission.)

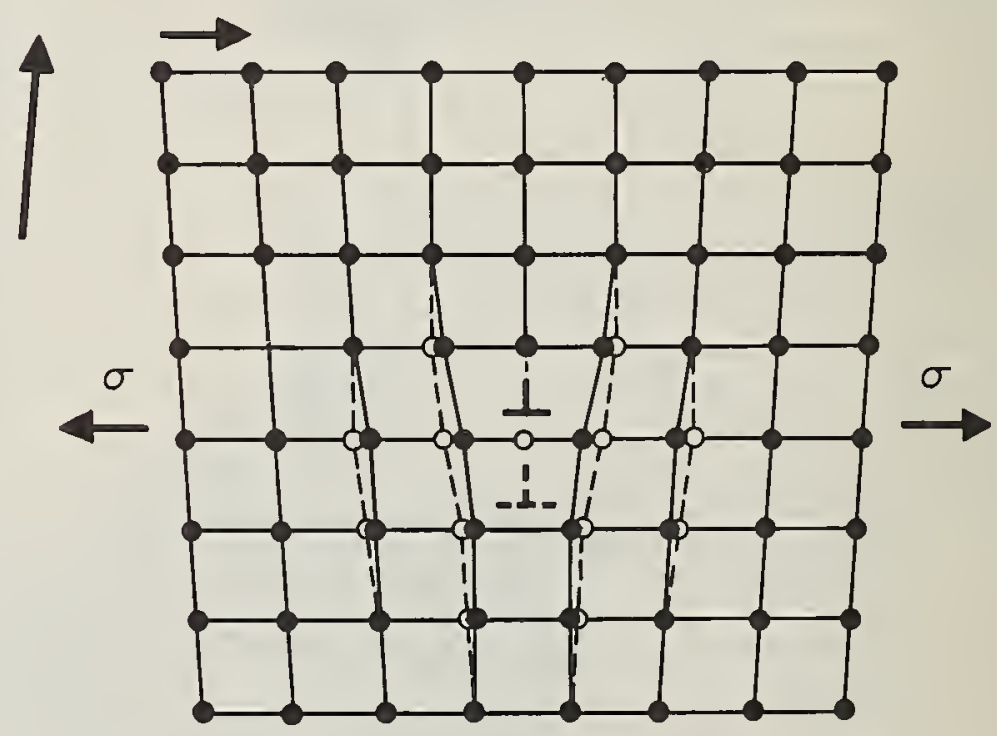

FIGURE 21. An edge dislocation moves one interatomic spacing to the right on its slip plane by glide. The solid circles represent the positions of the atoms before motion, the open circles, the positions after. The sheer stress $T$ is responsible for the glide. (Adapted from Dislocations in Crystals by W. T. Read, Jr., copyright 1953 , McGraw-Hili Book Co., Inc. Used by permission.)

FIGURE 22. An edge dislocation moves one interatomic spacing down to the next slip plane by climb. The solid and open circles represent, respectively, the positions before and after motion. The tensile stress $\sigma$ is responsible for the climb. 


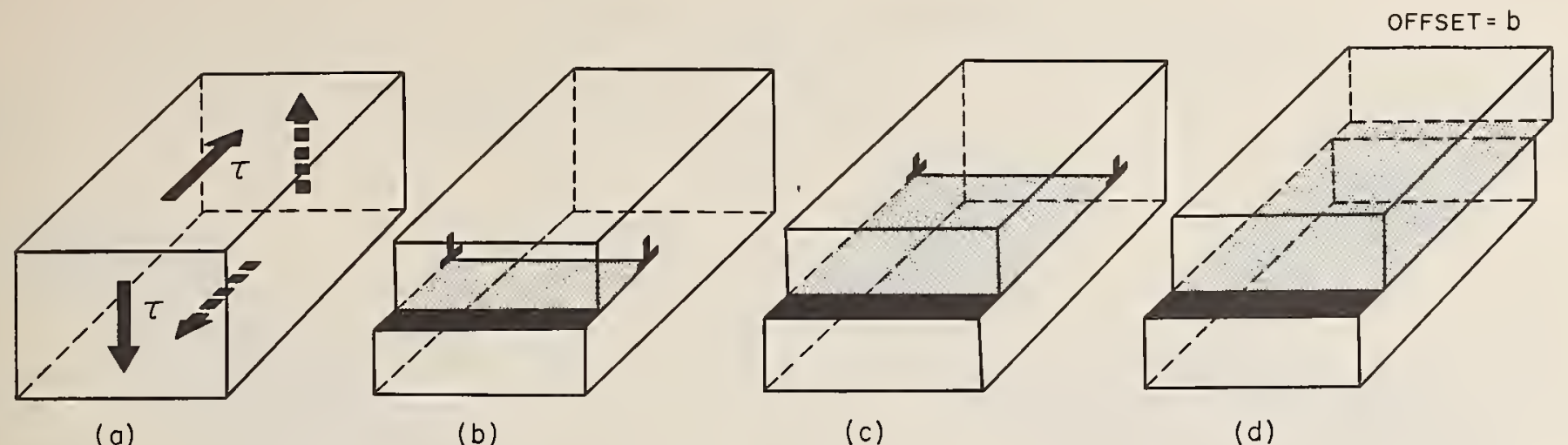

(a)

(b)

(c)

(d)

FIGURE 23. Macroscopic deformation from edge dislocation glide. When the dislocation moves on its slip plane, the crystal deforms; an applied stress can do work in the deformation. (a) The applied shear stress that does work when the dislocation of figure 21 glides. (b) An edge dislocation, coming in from the front face of the crysta1, has moved one-third of the way across the slip plane. (c) The same, except that the dislocation has moved two-thirds of the way across. (d) The dislocation has moved all the way across; the offset of the upper surface relative to the lower is equal to the slip vector $\underline{b}$.

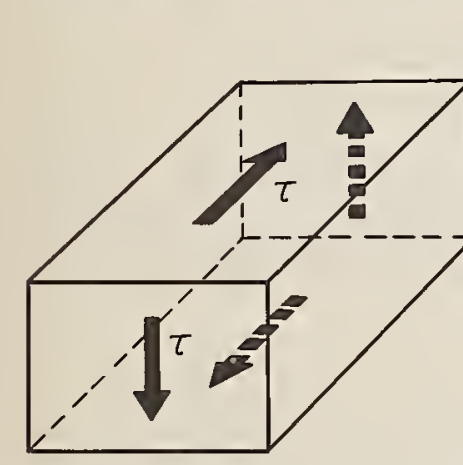

(a)

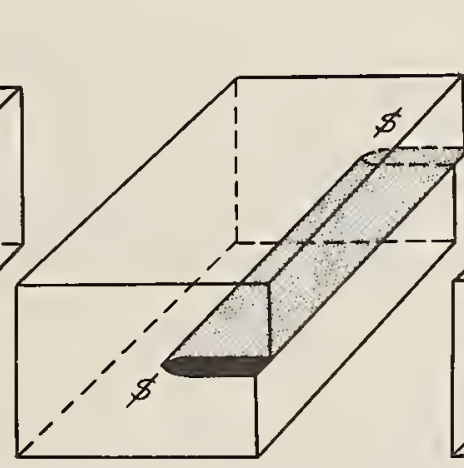

(b)

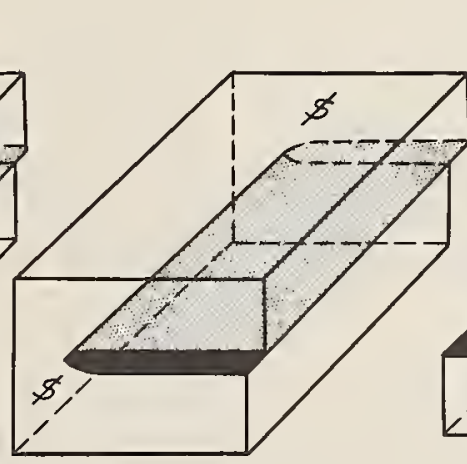

(c)

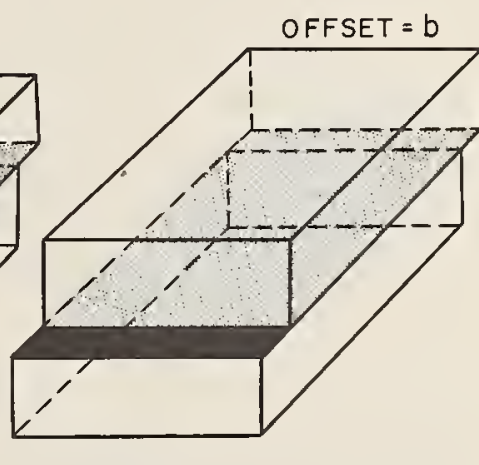

(d)

FIGURE 24. Macroscopic deformation from screw dislocation glide. (a) The same applied shear stress as in figure 23(a). (b) A screw dislocation, coming in from the right face, has moved one-third of the way across the slip plane. The symbol $\$$ denotes the screw dislocation. (c) The same, except that the dislocation has moved two-thirds of the way across. (d) The dislocation has moved all the way across; the offset $\underline{b}$ is the same as in figure $23(d)$.

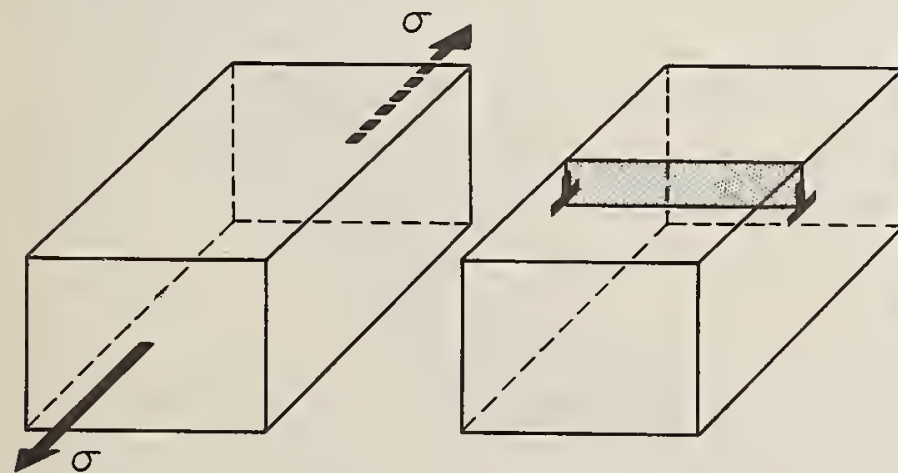

(a) (b)

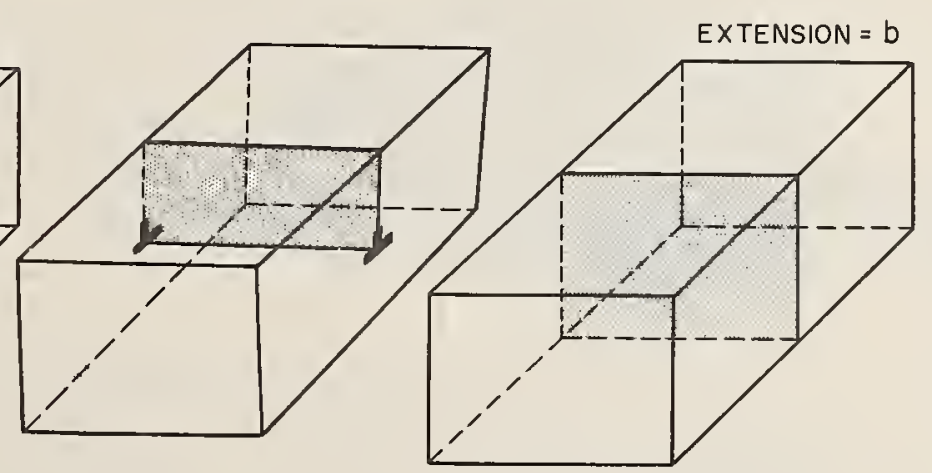

(c)

(d)

FIGURE 25. Macroscopic deformation from edge dislocation climb. (a) The applied tensile stress that does work when the dislocation in figure.22 climbs. (b) An edge dislocation, coming in from the upper face, has climbed one-third of the way down into the crystal. (c) The same, except that the dislocation has climbed two-thirds of the way down. (d) The dislocation has climbed all the way through the crystal; as a result, an extra atomic plane (shaded) has been added to the crystal, displacing the back face by a distance $\underline{b}$ relative to the front face of the crystal. 


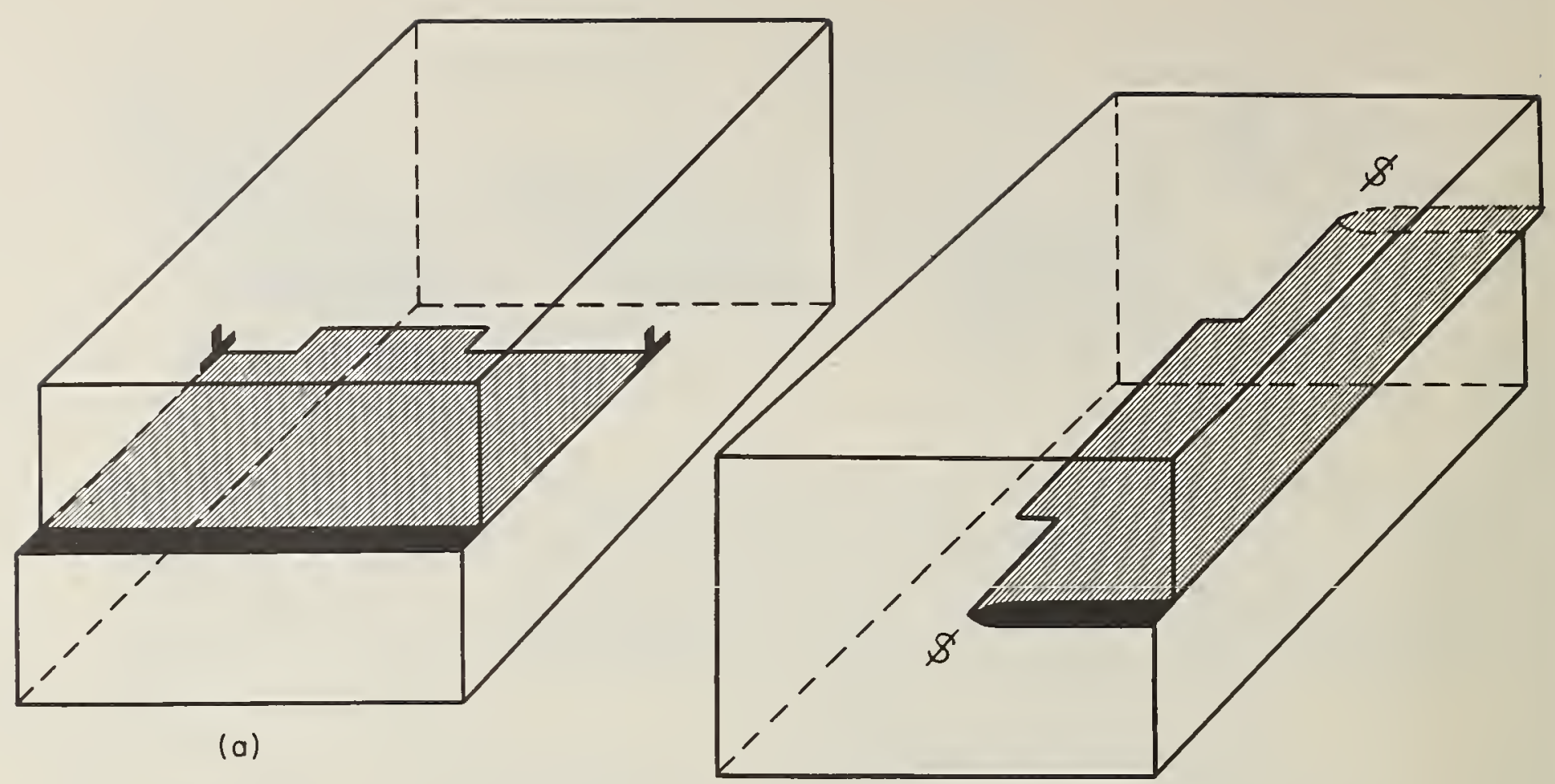

(b)

FIGURE 26. Kinks in dislocations. The kink is an offset of the dislocation in the slip plane. (a) Edge dislocation with two kinks. (b) Screw dislocation with two kinks.

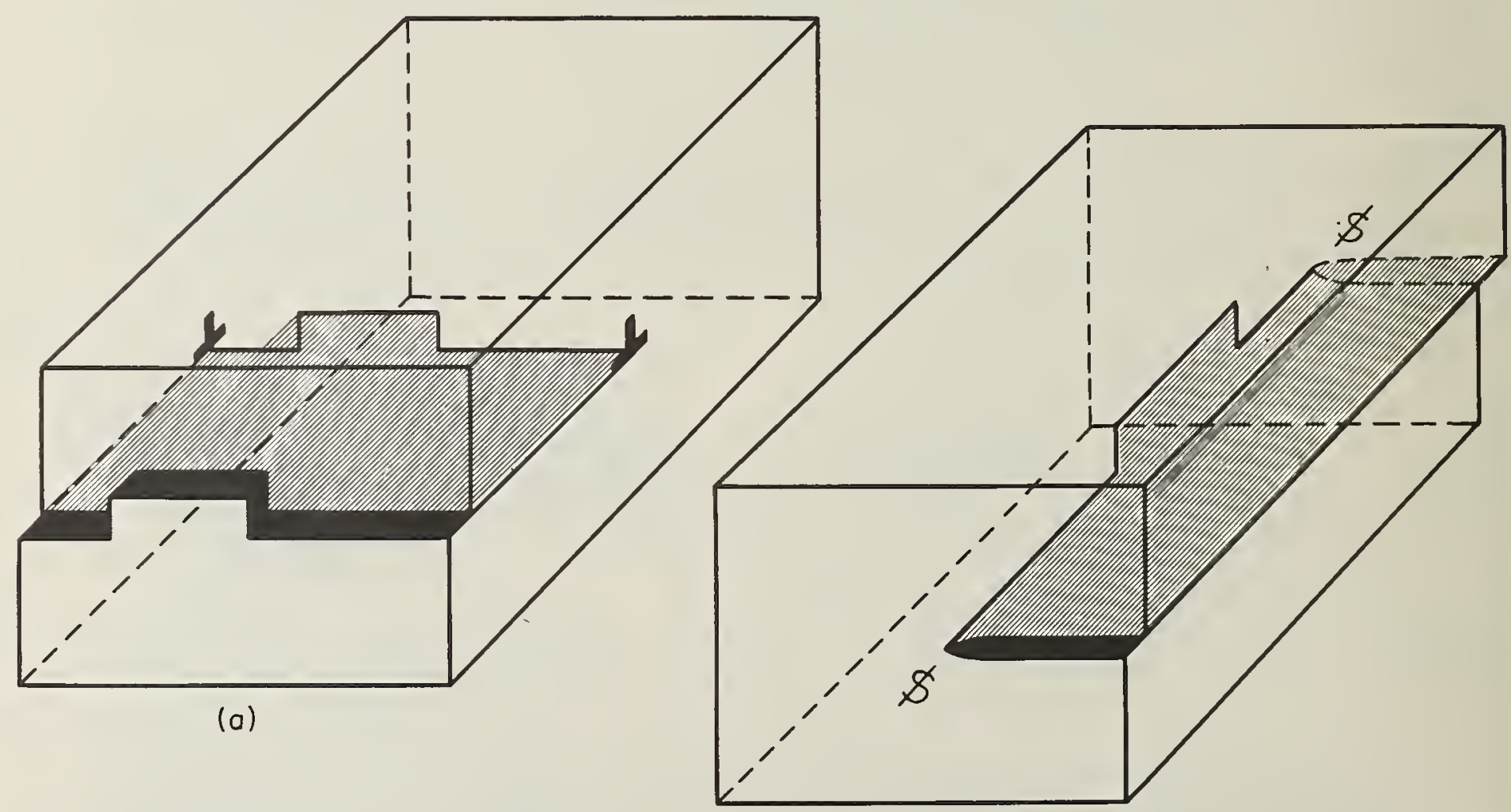

(b)

FIGURE 27. Jogs in dislocations. The jog is an offset of the dislocation not in the slip plane. (a) Edge dislocation with two jogs. (b) Screw dislocation with two jogs. 


\author{
H. G. F. Wilsdorf \\ The Franklin Institute Laboratories \\ Philadelphia 3, Pennsylvania
}

\title{
1. Experimental Techniques
}

\subsection{Introduction}

Methods for the direct examination of dislocations have only been developed since 1953, but dislocation theory had already existed for two more decades. Thus, as soon as each experimental method became available, it was immediately employed to test the various tenets of dislocation theory. This process is still going on, but the theoretical predictions concerning the basic properties of dislocations have now been confirmed.

Above all, the fundamental hypothesis that glide deformation in crystals is propagated by moving dislocations, each associated with a smallest unit of slip comparable to atomic distances, needed confirmation. The first part of this proof came comparatively late in the whole development, and only with the advent of diffraction electron microscopy. Several motion pictures were prepared within a short period of time showing dark lines which were moving at varying speeds in thin metal foils a few thousand Angstroms thick $[1,2]$. ' However, much basic information had already been obtained earlier by other means.

It is the aim of this paper, to describe the experimental techniques which have made the most significant contributions and to discuss their applications and Iimitations. Taking into account the purpose of this series of lectures, the reader will not expect completeness of literature quotations; however, his attention will be drawn to more detailed articles which do exist for some of the methods described. Concerning the properties of dislocations, as deduced by theoretical considerations, reference is made to the preceding article by R. dewit on "Theory of Dislocations."

\subsection{Decoration Techniques}

The early attempts to study dislocations experimentally were made with light microscopes. In these the investigator was greatly concerned with "magnifying" dis locations by some means in order to bring these atomistic line defects into the range of his instrument's resolving power. One powerful method of achieving this end makes use of the known fact that foreign atoms are drawn into the cores of dislocations [3]. In edge dislocations, atoms larger than those of the host crystal are attracted to the regions of dilatations, small atoms into the regions of compression expansion. In this way the free lattice energy is reduced. Although normal stresses are absent in the stress field of screw dislocations, these, too, attract foreign atoms, particularly when extended, since their stress fields may be reduced by the shear strains of interstitial foreign atoms [4], and because their free energy may be reduced in the atomic layers bordering the stacking fault [5]. The predicted accumulation of impurity atoms at dislocation lines as well as the high stresses in their immediate vicinity make dislocations the preferred nucleation sites for precipitates. As a consequence, by controlling the nucleation and growth of precipitates through proper heat treatments, it is possible to "decorate" the dislocations with particles which are large enough to be seen with a light microscope, but still small enough to afford the requisite resolution. The techniques of observation as well as the precipitation methods vary considerably as to whether the specimens are ionic crystals, semiconductors, or metallic crystals, and, therefore, they w111 be treated separately for these three classes of materials.

The optical transparency of most ionic crystals represents a considerable advantage since it makes it possible to see decorated dislocation lines in their threedimensional distribution. Partly for this reason, dislocations in alkali and other metal halides have been studied by many investigators. This whole field of investigation was first opened up by Hedges and Mitchel1 [6], who discovered in 1953 that subboundaries in silver bromide single crystals can be made visible and appear as parallel lines or networks after suitable treatments. Figure 1 is one of their later micrographs and is representative of the beautiful results obtained by Mitchell and his group. The method employed to decorate the dislocations is as follows: Crystals,

${ }^{1}$ Figures in brackets indicate the literature references on page 48 . 
studied either after deformation or in the as-grown state, are subjected to a heat treatment $\left(\sim 350^{\circ} \mathrm{C}\right)$ in bromine vapor under pressure of a few atmospheres, for various lengths of time, and then cooled rapidly. After a subsequent short exposure to filtered light, photolytic silver appears along dislocation lines. The decoration process is effective within a $40 \mu$ thick layer from the surface, and thin wafers may be cleaved from the crystal surface for detailed light microscopical studies of dislocation subboundaries and dislocation reactions.

A few years later Amelinckx [7] described similar experiments on sodium chloride. Figure 2 11lustrates the essential features of his preparation technique. A hole is drilled into a single crystal of $\mathrm{NaCl}$ and filled with sodium. After annealing the crystal at a temperature of $750{ }^{\circ} \mathrm{C}$, many fine precipitates of sodium are observed distributed throughout area $B$ of the specimen (indicated by dots in figure 2) but are absent in area $A$. At the boundary between $A$ and $B$, preferred precipitation, not masked by random precipitation, is found along dislocation lines. They are studied in thin wafers cleaved from the interface area C. An example of decorated dislocations is shown in figure 3.

Amelinckx [7] has summarized considerations on a decoration mechanism which apparently causes the segregation of colloidal sodium at dislocation lines. On the arrival of F-centers at jogs, sodium ions are belleved to be neutralized, and rows of sodium atoms along the dislocation lines are formed as a consequence. High mobility of sodium atoms along the dislocation axis leads to segregation as the supersaturation of $\mathrm{F}$-centers increases with continued cooling. However, during cooling from as high a temperature as $750{ }^{\circ} \mathrm{C}$, there exists also a supersaturation of vacancies. Pratt [8] has elaborated on the decoration process by considering the arrival of vacancies at negatively charged jogs. It is a feature of both mechanisms that the sodium in contact with the crystal provides chlorine vacancies and electrons that move through the crystal and are finaliy attracted to dislocations.

These early papers stimulated much work to improve decoration techniques for dislocations in ionic crystals, and many attempts have been made to eliminate or to reduce the major shortcomings of the technique, namely the high annealing temperature and the necessity of smali impurity concentrations in the original crystal. Mitchell with his coworkers and the School at Gent have achieved many improvements in techniques $[9,10,11,12]$ and extended them to a number of alkali and silver halides [13,14]. These studies have ylelded valuable information on dislocation boundaries and dislocation reactions $[15,16,17,18,19,20,21]$. Most recently, Childs and Slifkin [22] succeeded in decorating dislocations in large crystals of silver chloride by subjecting the specimens to short light pulses with a synchronous driving electric fleld. This procedure [23] produces electrons which are swept into the crystal and, when trapped, convert silver ions into atoms. Aging at room temperature results in the decoration of dislocations.

Silicon, a semiconductor, although opaque to visible light, is transparent to infrared light, and it is this property which made it possible to study decorated dislocations in this material by a novel technique. The method was developed by Dash, employing a microscope in conjunction with an infrared image tube [24]. The decoration of dislocations occurs in silicon crystals of about $1 \mathrm{~mm}$ in thickness, when the etched crystal, whose surface has been wetted with a dilute copper nitrate solution, is heated at temperatures around $1,200{ }^{\circ} \mathrm{C}$ for about an hour and then cooled within minutes. Copper diffuses into the crystal during the heat treatment, and during the period of rather rapid cooling small precipitates form along the dislocations. The size of the particles (a few microns) and the resolving power of the infrared equipment limit the resolution of the method severely. In this respect it is fortunate that single crystals of Si can be grown with very low dislocation densities [25], and the most interesting results have been obtained in specimens with $\rho \sim 10^{4} \mathrm{~cm}^{-2}$.

Figure 4 is an example of the results that have been obtained. This particular micrograph is of spectal interest since, at the same time, it is proof for a $1: 1$ correlation between dislocations and etch pits, which will be discussed in the following chapter. Recently, the infrared technique was applied to a detalled study of the climb of dislocations induced by the diffusion of gold into silicon [26].

In applying the decoration principle to metals, it becomes necessary to add one more step to the preparation procedure. Optical observations on metals are 1imited to their surfaces, and the minute precipitates, present where decorated dislocations intersect surfaces, cannot be distinguished from the matrix with a light microscope. This fact makes it imperative either to etch the specimens or to make replicas of electropolished surfaces, suitable for investigation with the electron microscope, after the decoration treatment. 
It had been observed as early as 1936 that indistinct rows of precipitates sometimes formed parallel to slip planes in aluminum rich aluminum-copper alloys, and additional work confirmed that preferred precipitation may take place in slip bands [27,28, 29]. Later Castaing and Guinier [30] and Lacombe and Berghezan [31], partly using replicas examined in the electron microscope, detected sub-boundaries which were outlined by copper rich precipitates in A1-4 percent $\mathrm{Cu}$. In 1954 Wilsdorf and KuhlmannWilsdorf [32] decorated dislocations in deformed polycrystalline A1-2 percent Cu and provided for the first time experimental evidence for single dislocations in a metal. Moreover, they found segments of concentric loops outlined by fine precipitates and identified them as sequences of dislocations emitted by a dislocation source (figure 5). In further similar work these authors [33], as wel1 as Thomas and Nutting [34] and Takeyama and Koda [35], investigated decorated single dislocations in networks and low angle boundaries in aluminum-copper alloys. A few years later Jaquet and Weil1 [36], using the same technique, published additional evidence for the operation of Frank-Read dislocation sources within grains of Al-4 percent $\mathrm{Cu}$. The technique yielded only very limited results on dislocation arrangements in slip bands.

The decoration and preparation of specimens used in all of the above investigations proceeded in the following sequence: (1) rapid water quench after solution treatment at $530{ }^{\circ} \mathrm{C}$; (2) plastic deformation; (3) anneal at $180^{\circ} \mathrm{C}$ (or up to $230{ }^{\circ} \mathrm{C}$ ) for a few hours; (4) electrolytic polish-etch; (5) replication for examination with electron microscope. Although the technique is limited to surface observations, it is also suitable for obtaining information on dislocations within crystals, since the specimens can be electropolished progressively.

Another alloy, 3 percent silicon-iron, has been extensively studied by a decoration-etch pit technique $[37,38]$. Suits and Low found that the decoration of dislocations in this material was due to the segregation of carbon [39]. An amount of 0.0005 atomic percent $\mathrm{C}$ and a heat treatment at $160^{\circ} \mathrm{C}$ for $15 \mathrm{~min}$ are sufficient to decorate dislocations to the extent that they can be detected by a subsequent etch, even though no precipitates have formed on them. Studies of dislocations introduced during deformation or growth produced interesting results on polygonization and small angle boundaries. Later, Low and Guard $[40]$ succeeded in observing dislocation arrangements in active slip planes by replicating surfaces parallel to these planes.

The decoration and etching of dislocations in a $70 / 30$ brass (again without the formation of actual precipitates) is possible when 0.6 percent cadmium is added to the melt [41]. Quenching from $600{ }^{\circ} \mathrm{C}$, deformation, annealing for a few hours at $200{ }^{\circ} \mathrm{C}$ and electro-etching results in patterns as illustrated by figure 6 . The small size of the etch pits obtained in conjunction with the use of replicas for electron microscopy allows a very high resolution, namely, up to dislocation densities of $p=10^{10} \mathrm{~cm}^{-2}$. The results obtained from these studies range from information on dislocation networks and subboundaries to the distribution of dislocations on active slip planes and to measurements of dislocation densities and other parameters of importance to the theory of plastic flow in metals [42].

Cadmium added in amounts of 0.1 percent to zinc is effective in decorating dislocations in growth boundaries $[43,44,45]$ which formed during solidification. The micrographs taken with the light microscope (see figure 7) are somewhat similar in appearance to those shown in figures 1 and 3 obtained on ionic crystals in transmitted 1 ight. However, while the lines seen in the latter are due to single dislocations, the dotted lines in figure 7 represent the intersection of boundaries with the surface, which generally are not resolved into single dislocations. A subsequent refinement permitted Damiano [46] to decorate and etch single dislocations (see figure 8). While the spatial distribution of decorated dislocations in ionic crystals can be investigated simply by moving the colum of the microscope, complex techniques had to be devised for similar studies on metals. One of these, for the continuous recording of etch patterns [47], will be described in section 1.3. Also, the electron microscope has been successfully employed when it became possible to keep the size of etch pits small enough, i.e., $\sim 0.5 \mu$.

\subsection{Etching Techniques}

Efforts to mark the emergence of dislocations at surfaces represent refinements of etching techniques which had been known for many decades to crystallographers and metallurgists. If a crystal sphere is etched with a suitable reagent, its surface is attacked with varying speed, depending on the differences in chemical potential between surface atoms in crystallographic planes of different orientations. 
Similarly, it is possible to reveal even undecorated dislocations by means of etching, since the chemical potential of atoms at the point of emergence of a dislocation at the surface is higher than those at a perfect surface. Experience has shown that under suitable conditions etch pits may be formed at edge as well as at screw dislocations. Since the shear strains in the elastic stress field of a screw dislocation are zero at a free surface, it thus appears certain that it is not the elastic energy but the core energy of a dislocation which provides the necessary increase in free energy for preferential etching at dislocation sites [48].

It is generally agreed that the removal of atoms from a crystal surface proceeds along atomistic steps which contain kinks [49]. The first requirement for the production of isolated etch pits at dislocations is, then, the preferential formation of two-dimensional nuclei at dislocation sites, and the second requirement is a suitable ratio between the removal rate of atoms from the steps, $V_{S}$, and the nucleation rate of new steps, $V_{n}$. According to Johnston [50], the condit lon for producing visible pits is satisfied when $\mathrm{v}_{\mathrm{s}} / \mathrm{v}_{\mathrm{n}}$ is smaller than 10 . In controlling this ratio one is obviously limited in changing $\mathrm{V}_{n}$, since the difference in chemical potential between a perfect surface and a surface ${ }^{n}$ at the point of a dislocation's emergence is small. Therefore, attempts have been made to affect the rate of dissolution along atomistic steps. Gilman, Johnston, and Sears [51] have shown experimentally that the etch pit formation at dislocation sites in LiF can be influenced by changing the under-saturation of the etchant as well as by adding foreign ions to it. They found that distilled water with an addition of about $2 \times 10^{-6}$ mole fraction of $\mathrm{FeF}_{3}$ was the most effective etchant, and that above 25 percent LiF saturation the solution ceased to etch preferentially at dislocation sites. Recently, Ives and Hirth have studied the dissolution kinetics of dislocation etch pits in LiF and determined the variables -- time, temperature, undersaturation, crystal perfection, and orientation [52]. Similar studies have been carried out on Ge by Riessler [53]. Theoretical considerations on etching at dislocation sites may be found in papers by Cabrera et al. $[54,55,56]$.

The major prerequisite for producing etch pits preferentially at dislocation sites is a very smooth and clean surface. For most metals and alloys this condition cannot be satisfied easily, and chemical and electrolytic polishing techniques are frequently employed. Ideal surfaces for this purpose, however, can be obtained by cleavage. It is rather fortunate that many ionic crystals cleave easily and that these cleavage surfaces are not parallel to active slip planes. On metals, etching solutions attack the surface at dislocation sites only if they are parallel within a few degrees to low indexed planes. This is understandable from Cabrera's analysis [55], because nonclosepacked planes consist practically of monoatomic steps, and the dissolution proceeds without the necessity of forming nuclei. In fact, ali detailed results, so far, on pure metals have been obtained on $\{111\}$ planes $^{2}$, which indicates a severe limitation of the etching technique as applied to metals.

The shape of etch pits allows conclusions to be drawn regarding certain characteristics of dislocations. Primarily, the shape of a dislocation etch pit reflects the symmetry of the respective surface, provided a suitable etchant is applied. Deviations from symetry, however, may be interpreted in terms of the orientation of the dislocation at the surface. Figure 9 a shows a drawing of square pits which may be obtained on a $\{100\}$ plane of NaCl. The pits are symetrical with regard to their apex, and it can be deduced that dislocations meet the surface perpendicularly. On the other hand, if dislocation lines include an angle $\alpha$ with the normal to the surface, one finds a, being the distance from the apex to the symmetry center of the pit, related to d, the depth of the pit as $\alpha=a / d$ (see figure $9 \mathrm{~b}$ ). The arrangements of pits in rows at regular intervals indicate in most cases the presence of a dislocation boundary or a hexagonal network. The latter would show pits with the apex shifted to one side in alternating fashion, as drawn in figure 9c. Concerning the analysis of tilt and twist boundaries, Amelinckx [57] was able to distinguish between screw and edge dislocations, since they gave rise to pits of different depth. Gilman, Johnston, and Sears [51] confirmed this result on LiF showing explicitly that edge dislocations produce deeper pits than screw dislocations.

\footnotetext{
${ }^{2}$ However, F. W. Young has been able to obtain etch pits in copper on $\{100\}$ and $\{110\}$ faces (J. Appi. Phys. 32, 192 (1961)).
} 
The depth of an etch pit, moreover, can also be influenced by the presence of impurities, to the extent that in many instances it has become possible to differentiate between "old" and "new" dislocations $[58,59]$. Depending on the experimental conditions (choice of etchant and particular effect of the impurities), "old" dislocations, i.e., dislocations which remain in the crystal after a heat treatment or which have been formed during solidification and which, by inference, contain impurity atmospheres, may be deeper or shallower than dislocations newly introduced by plastic flow. The decision as to whether "old" or "new" dislocations have given rise to etch pits can normally be made by evaluating their arrangements. Recently, Livingston [60] found that positive and negative edge dislocations produce etch pits of different depth on \{III\} planes of copper under carefully controlled conditions; again, the distribution of pits in conjunction with planned experiments on single crystals enabled the investigator to arrive at unambiguous conclusions. A further refinement in applying the etch pit technique to studies of dislocations during plastic flow in crystals allowed Gilman and Johnston [58] to follow the path of dislocations in LiF. Etching the specimens before and after straining produced etch pits of different shape, namely, the usual sharp pits and others with a flat bottom. The latter arise because a pit ceases to grow in depth after the dislocation is removed from its original site. Repeated etching after the dislocation has moved away will only widen the original pit, which now develops a flat bottom, while a sharp but smaller pit indicates the new site of the dislocation (figure 10). Equivalent experiments on copper were also successful $[61,62]$.

Earlier investigators were greatly interested in finding conclusive evidence as to whether or not there existed a $1: 1$ correspondence between etch pits and dislocations. Ionic crystals were found very suitable for such investigations and provided proof that in many, but not all, techniques, every pit marks the point of emergence of a dislocation and every dislocation is marked by a pit. One method used to investigate this point is as follows: After cleaving a deformed crystal, the two surfaces were etched, and the resulting patterns were compared. From the perfect agreement of the pit distribution (see figure 11), it was concluded that with suitable etchants the pits indicated the emergence of dislocations at the surface in a $1: 1$ ratio [58]. Another method is to etch ionic crystals after decorating the dislocations in them. The test procedures just described cannot be applied in general, and a comparison of dislocation density and dislocation patterns as shown by experiment with estimated data is usually made as a check.

Most etch pit studies have been carried out with light microscopes since it is very difficult to obtain etch pits with diameters less than $1_{\mu}$. This limits the application of etching techniques to crystals containing less than $10^{8}$ dislocations per $\mathrm{cm}^{2}$. For a number of applications it is most desirable to follow the progress of etch patterns by cinemicrographic recording techniques [47]. Damiano and Herman [63] showed that the apparatus was capable of recording 2 frames per second and permitted meaningful enlargements of up to $200 x$.

To date, it is not possible to predict a successful etchant for dislocations, and no attempt will be made here to list the numerous solutions which have been described in the literature. There are a number of excellent review articles on the subject which contain detailed information and many literature references. Johnston's paper [50] specialized in non-metalif crystals and contains, in addition to 337 references, tables with etching solutions. A Russian review article [64] covers metals, semiconductors, and ionic crystals, and lists many etching solutions and 264 references.

The great value of the etching technique with regard to the exploration of specific properties of dislocations is obvious from the above discussion. In addition, studies of sub-structures, polygonization, fracture, dislocation movements and others have been aided by this technique, some of which will be mentioned in section 2 of this paper.

Other etching techniques, such as thermal etching $[65,66,67,68,69]$, ionic etching (sputtering) [70,71,72], and etch pit formation by the condensation of vacancies [73] have met with some success, but it appears at present that their application is rather limited.

\subsection{Diffraction Techniques}

$X$-rays and electrons are diffracted by crystal lattices. Atoms in a lattice are arranged in planes, and the direction of the diffracted beams is given by Bragg's law

$$
2 \cdot d \sin \theta=n \cdot \lambda,
$$


where $\mathrm{d}$ is the distance between the reflecting lattice planes, $\theta$ the angle subtended between those planes and the incident beam, $\lambda$ the wavelength, and $n$ is an integer. For exploring the lattice structure, it is equally of importance to know the intensity of the diffracted beams, which can be calculated with the help of the structure factor $\mathrm{F}$. In exponential form, $\mathrm{F}$ can be written as

$$
F_{h k 1}=\sum_{1}^{n} f_{n} \exp \left[2 \pi i\left(h u_{n}+k v_{n}+1 w_{n}\right)\right],
$$

$f$ being the atomic scattering factor, hk1 the indices of the reflection, and uvw indicating the coordinates of the atoms in the unit cel1. The above expression applies only to the perfect crystal lattice. Distortions of the lattice in the vicinity of dislocations can result in a change in the amplitude of the diffracted waves and hence in a change in the diffracted beam's intensity. This is the basis for the diffraction techniques discussed in the following paragraphs.

It is advantageous to treat the formation of dislocation images by $\mathrm{X}$-rays and electrons separately. Although the diffraction mechanism for X-rays and electrons is basically the same, there exists, however, a distinct difference in the image forming capability of X-rays and electrons. While the former cannot be focused properly by applying conventional optical principles, the path of electrons can be readily influenced by magnetic fields, and images of high resolution and quality may be obtained. Obviously, then, the experimental techniques for these two radiations in the study of single dislocations must be substantially different. Also, the penetration power through matter is much higher for X-rays than for electrons, so that specimens of different thickness have to be used.

\section{a. X-ray Techniques}

Considering the propagation of X-rays through crystals, one has to remember their high penetration power, which makes X-ray techniques a very suitable tool for studying dislocations within thick crystals. Diffracted X-ray beams, transmitted through single crystals, suffer an intensity loss which, for equal thickness, becomes larger with increasing lattice perfection. This attenuation of X-ray beams is known as "primary extinction" and is caused by interference between repeatedly reflected X-rays at the Bragg angle. Since, usually, the distorted crystal volume around a dislocation upsets the phase relationship between diffracted beams, X-rays passing close by dislocations are transmitted with much higher intensity than those passing through the perfect lattice. The result is that beams of parallel, monochromatic X-rays, being reflected from a set of crystal planes in an otherwise rather perfect crystal, exhibit zones of high intensities which are a direct projection of dislocations in the crystal.

The behavior of diffracted X-ray beams described above applies only if the Burgers vector has a normal component with respect to the reflecting lattice planes, as will be discussed with the help of figure 12 (an analytical description is beyond the scope of this article). The figure shows part of a dislocation loop in the simple cubic lattice with the screw component lying vertical and the edge component being parallel to the horizontal direction. It is immediately seen that -- at least within the accuracy of representation of this figure -- al1 planes containing the Burgers vector remain undistorted while those which deviate from this orientation are curved. The lattice curvature becomes larger the more normal the planes are with respect to the Burgers vector. Consequently, dislocations become visible only if the reflecting family of lattice planes makes a significant angle with the Burgers vector. This is a most important condition; moreover, it may be utilized to determine the dislocation's Burgers vector which cannot be simply obtained from micrographs. Conversely, in the majority of cases the glide plane of the dislocations is known or can be guessed. This then leaves the choice of three Burgers vectors in the case of $\{111\}$ slip planes in fcc crystals, or two $1 / 2<111>$ and one $<100>$ Burgers vectors for a $\{110\}$ slip plane in bcc, etc. In order to decide among the various possibilities, the specimen may be oriented for various Bragg angles until the dislocations in question become invisible, while others with different Burgers vectors must still appear on the photographic plate. In this way the Burgers vector can be determined, the knowledge of which often is of utmost importance for the analysis of dislocation patterns.

of the methods based on the above principles, the one to be described first is due to Lang $[74,75]$, who named it "projection topography." A schematic illustration of the technique is given in figure 13. A collimated, monochromatic X-ray beam of parallel rays is directed onto the crystal specimen, which is oriented for Bragg reflection. A lead screen prevents the primary beam from reaching the photographic plate, allowing only the diffracted beam to sensitize the film. In order to obtain 
a topograph of the whole crystal, the specimen and film are put on a carriage so that they may be moved together parallel to the crystal. The thickness of the specimen is in the order of $1 \mathrm{~mm}$. Great care has to be taken to produce smooth surfaces, since smal1 pits and scratches will show in the photographs and cannot be distinguished from the contrast caused by dislocations without additional experiments.

A perfect surface is even more important when using a reflection technique which is known as the "Berg-Barrett" technique (figure 14) [76]. Here a monochromatic Xray beam collimated by a narrow slit strikes the surface of a crystal, and the diffracted beam is reflected onto a photographic plate which has to be as close to the specimen as is possible. The latter condition is dictated by the fact that the diffracted X-rays, owing to the slightly divergent beam, are lying on a cone so that the image of a point in the specimen will appear as a short arc on the photographic plate. In order to keep this distortion to a minimum, the distance between photographic plate and specimen should be as small as possible. 'In principle, this applies also to Lang's technique.

Figures 15 and 16 illustrate the results which can be obtained with the transmission and reflection technique, respectively. In both photographs single dislocations are easily discernible and show up either as long lines or as slightly elongated spots. Figure 16, in addition, demonstrates a high density of dislocations in subboundaries. These two examples are suitable for estimating the resolution that may be attained. It is seen that, in average, the dislocations are recorded with a width of about $20 \mu$. In exceptional cases, dislocations separated 3 to $5 \mu$ can be detected [77] and this represents the ultimate obtainable. The conclusion can be drawn that $\mathrm{X}$-ray techniques may resolve dislocation patterns at densities of not more than $10^{5}$ to $10^{6} \mathrm{~cm}^{-2}$ and, therefore, are largely restricted to the exploration of dislocation arrangements in as-grown or annealed crystals.

The major advantages of using X-ray techniques are (1) that the investigation is non-destructive; (2) that thick crystals $(0.3-2 \mathrm{~mm})$ and large areas $\left(1 \mathrm{~cm}^{2}\right)$ can be examined; (3) that the Burgers vector of the dislocations can be determined with relative ease; and (4) that it is possible to produce stereo-topographs.

\section{b. Diffraction Electron Microscopy}

During the past five years the electron microscope has become the most prominent tool for the experimental study of dislocations. This development started with the observations of Bollmann [78] and Hirsch, Horne and Whelan [1] in 1956 who recognized that certain contrast effects obtained from thin metal foils were caused by stacking faults and dislocations.

The contrast in electron micrographs of amorphous specimens is due to electron scattering, which depends on differences of the specimen's atomic composition and specimen thickness. If the specimen is crystalline, additional contrast may be produced by interference phenomena between electron waves. Since objective apertures in electron microscopes are in the order of $10^{-3} \mathrm{rad}$, even first-order diffracted beams are not permitted to contribute to the final image, as illustrated in figure 17 . An electron beam, coming from the condenser, passes through a single crystal which is buckled in a central portion. Due to the buckling, a certain zone of the crystal is accidentally oriented so that some low-index plane makes the Bragg angle with the incident electron beam. In this zone electrons are diffracted out of the primary beam and are held back by the objective aperture. The photographic plate shows an underexposed area where electrons have been reflected out of the image forming beam corresponding to the projection of the diffracting specimen volume. Such bands or broad lines are called "extinction contours"; they can be seen to move on the fluorescent screen when the specimen is tilted and indicate that crystal volume which happens to be oriented for diffraction.

The path of electrons responsible for dislocation images is similar to those shown in figure 17. However, the contrast produced by dislocations is due to phase changes of the incident electron waves, as may be seen by the following consideration: Employing the concept of the "reciprocal lattice," formula (1) can be written as

$$
A=\left|F_{h k 1}\right|=\sum_{n} f_{n} \exp \left[\begin{array}{llll}
-2 \pi & i\left(k-k_{0}\right) & r_{n}
\end{array}\right] \text {, }
$$

where $f_{n}$ is now the scattering factor for electrons, $k$ and $k$ are the wave vectors

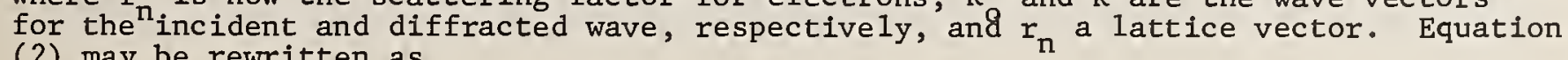
(2) may be rewritten as 


$$
A=\sum_{n} f_{n} \exp \left[-2 \pi i(g+s) r_{n}\right]
$$

(see the drawing, figure 18). The atomic displacements around a dislocation are now taken into account by a phase factor a so that the amplitude of a diffracted wave in an imperfect crystal is given by

$$
A=\sum_{n} f_{n} \exp (-2 \pi i s \cdot r) \cdot \exp (-i \alpha)
$$

The phase factor $\alpha=2 \pi g x$, where $x$ represents the displacement vector from the ideal lattice position. Inserting the proper value for the displacement vector $x$ and integrating over the diffracting crystal volume yields the amplitude of the diffracted wave. For a detailed analytical treatment the reader is referred to a recent book by Thomas [79] who has summarized the relevant work by Hirsch, Howie and Whelan.

Figure 19a shows an example of the appearance of dislocations in an electron micrograph taken from a thin foil of stainless steel. It can be seen that the "image" of the dislocations has a width of $100-200 \AA$, and further that the intensity distribution along the dislocation line varies considerably, often giving rise to a dotted appearance. This is true, however, only for dislocations which are inclined against the incident beam, and it should be pointed out that the dottiness in the dislocation contrast does not allow us to draw conclusions with regard to the dislocation's fine structure. The effect is due to a diffraction phenomenon which can be explained only by the dynamic theory of electron diffraction. The spatial arrangement of the dislocations in figure $19 \mathrm{a}$ has been drawn in figure 19b.

The technique is also capable of determining the presence and position of single stacking faults as well as of sub-boundaries and grain boundaries which are inclined against the foil normal. Examples of the appearance of these lattice defects are shown in figures 20 and 21. Stacking faults as observed in figure 20 are rarely produced by plastic deformation of bulk samples but often occur when the dislocations move in thin foils. In equilibrium, stacking fault ribbons of extended glide dislocations in most pure metals have a width of less than $100 \mathrm{~A}$ and cannot be made visible by this technique, except, sometimes, as little triangles in extended nodes formed by dislocation networks. Examples of stacking faults in nonmetals will be given in section 2.

The preparation of specimens for transmission electron microscopy is difficult. Three rather stringent requirements have to be satisfied: (1) specimen thickness has to be in the order of a few thousand angstroms and should be uniform; (2) the surfaces should be atomistically smooth and may not be covered with irregular surface films; (3) the preparation has to be done with great care so as not to disturb the dislocation arrangement in the specimen.

The technique most commonly used for preparing the thin films is electromachining and electropolishing. A smali piece $(3 \times 3 \mathrm{~mm})$ is cut by a narrow liquid beam from the bulk sample, shaped to fit the specimen holder and finally "polished" with a circular jet of electrolyte directed to its center in order to produce the thin part in the specimen which will be used for observation with the electron microscope. An apparatus for final polishing is illustrated in figure 22. A great number of techniques for metals and ionic crystals have been described in the literature [79]; for a summary of the techniques used at The Franklin Institute Laboratories for many years the reader is referred to an article by strutt [80].

As might be expected, electron diffraction microscopy is predominantly employed for studying dislocations and dislocation behavior in bulk crystals. However, attention should be drawn to a unique feature of the technique, namely, the possibility of observing directly the dislocations in motion. This can be achieved by increasing the intensity of the electron beam [1] and thereby inducing undefined stresses on the specimen, or by actually straining a thin crystal in the electron microscope [81]. The different makes of electron microscopes require custom made straining devices because of the different specimen stage designs. A straining device for the Philips EM $100 \mathrm{~B}$ is shown in figure 23 [82].

Other auxiliary devices which enable the experimentalist to influence the dislocation patterns in the microscope include heating and cooling stages [79], as well as a combination of a heating stage with a straining device [83]. It has been tempting 
to record the dynamic behavior of dislocations by cine techniques, and motion picture studies on the mobility of dislocations in strained foils [1,2] and on twin formation [84] have been made.

The applications of diffraction electron microscopy to problems in materials science are more varied than those of any other technique mentioned above. Considering the high resolution for dislocations, which can exceed $150 \AA$, this is hardiy surprising. On the other hand, the limitation of "seeing" dislocations in a small area of only, say $10 \mu^{3}$, makes it difficult to produce reliable dislocation density measurements. Statements regarding the dislocation distribution of bulk specimens have to be based on hundreds of electron micrographs taken from many specimens. Since the preparation of the actual specimens is delicate and often cumbersome, comprehensive studies carried out with this technique are time consuming.

Critics of the technique have questioned the reliability of the information that can be gained by diffraction electron microscopy. It can be reasoned that "image forces" [85] will move dislocations during the thinning process out to the surface so that the dislocation density is reduced, or that at least rearrangements of dislocations will occur. Schmitz and Wilsdorf [86] have investigated this problem experimentally and found that the essential features of dislocation patterns in aluminum crystals were retained if the thickness of the thin specimens was $2000 \AA$ or more. If the thickness was reduced to values below $1500 \AA$, dislocations straightened out, oriented themselves to be perpendicular to the surface, and small prismatic dislocation loops were absent. For different materials, different minimum thicknesses are indicated. Since all commercially made electron microscopes are limited in the acceleration voltage to $10^{5} \mathrm{v}$, it may be rather difficult to obtain reliable results on dislocation patterns in metals with high atomic number. The development of electron microscopes with higher penetration power is urgently needed. Very clear micrographs from stainless stee $12-3 \mu$ thick have been taken by Dupouy and Perrier with their 1,000,000 v electron microscope $[87]$.

\section{Applications \\ 2.1. Introduction}

In this chapter some of the most important experimental evidence which has been accumulated during the past years will be reviewed.

Beginning with a description of results relating to the most fundamental properties of dislocations, the discussion will then concentrate on the behavior of glide dislocations, since our understanding of plastic flow in crystals has predominantly profited from experimental dislocation studies.

Only results obtained by decoration, etch pit, and diffraction techniques will be given. There are other methods which have been used for the investigation of the dislocation's stress field: for example, by Bond and Andrus working with polarized infrared light [88]; or the beautiful investigations, using high resolution diffraction microscopy, by Menter and his coworkers, who have resolved lattice planes in certain crystals and studied the fine structure of dislocations in very thin films [89,90]; or the elegant technique of field ion microscopy by Muller who achieves magnifications of more than $10^{6}$ with a resolution of about $3 \AA$ and observed not only dislocations but also single point defects on metal surfaces [91]. However, these are very specialized studies, and it remains to be seen how general their application may become.

\subsection{Evidence on the Burgers Vectors of Perfect Dislocations}

In a historical experiment, Voge1, Pfann, Corey, and Thomas [92] examined a row of almost uniformly spaced etch pits on germanium, representing as it turned out, a low angle tilt boundary. The lattice misorientation due to a subboundary can be calculated from the assumption that the etch pits represented single edge dislocations with $1 / 2<110>$ Burgers vectors. The angle of misfit $\theta \approx b / h$, where $h$ is the distance between two dislocations in the boundary and $b$, the Burgers vector [93]. Comparing the result obtained by the etch pit technique (figure 24) with an independent measurement of the lattice tilt using a conventional x-ray method, the agreement was found to be excellent, as may be seen from figure 25 [94]. This experiment gave proof that the theoretical predictions regarding the Burgers vector of perfect dislocations was correct -- at least for edge dislocations in germanium.

The above result has since been substantiated with respect to the Burgers vectors of glide dislocations in many different substances. 


\subsection{Evidence on the Stress Field of Perfect Dislocations}

The same experiment by Voge1, Pfann, Corey, and Thomas [92] also represents evidence that the stress field of dislocations is as derived theoretically: It is in accordance with the theoretical result that parallel edge dislocations assemble into uniformly spaced arrays, normal to their common Burgers vector, constituting tilt boundaries.

Figure 26 illustrates this point further. Here, part of this kind of a dislocation boundary, named "polygonization wall" [95], is seen in an electron transparent foil in aluminum. During the time of observation dislocations were captured in the boundary so as to accommodate the additional ones. Note, for example, the dislocation "pair," almost symmetrical with respect to the boundary.

However, investigations on polygonization were first performed much earlier [96]. Although not resolving individual dislocations, the earliest results already were in such excellent agreement with predictions that, even then, little doubt remained that the vertical arrangement of edge dislocations into walls represented an equilibrium position with respect to glide.

The perfection of low-angle boundaries in recrystallized metals depends, of course, on the annealing treatment. Figure 27 gives an impression of subboundaries in $\mathrm{A} 1-2 \%$ $\mathrm{Cu}$, affer a heat treatment of $1 \mathrm{hr}$ at $530{ }^{\circ} \mathrm{C}$.

Additional evidence to show that the stress field of dislocations in their own slip planes was also exactly as derived from elasticity theory came from studies of isolated dislocation pileups, i.e., groups of dislocations on one slip plane pushed against an obstacle (figure 28). The resultant shear stress on any one dislocation in the pileup resulting from the others is given by

$$
\tau_{j}=A \sum_{\substack{i=1 \\ i \neq j}}^{\substack{i=n \\ x_{j}-x_{i}}},
$$

the dislocations being numbered $1,2, \ldots \ldots i, \ldots n$, with the first one at $x=0 ; A=$ $\frac{\mu b}{2 \pi}$ for screw dislocations and $A=\frac{\mu b}{2 \pi(1-\nu)}$ for edge dislocations; $\mu$ is the modulus of rigidity, $b$ the Burgers vector, and $\nu$ Poisson's ratio. Although the problem cannot be solved for the general case, Eshelby, Frank, and Nabarro [97] have proposed a satisfactory estimate, provided the pileup contains many dislocations:

$$
x_{i}=\frac{A \pi^{2}}{8 n \tau}(i-1)^{2}
$$

Figure 29 shows the positions of the dislocations as found by a decoration-etch pit technique in $a$-brass [42] together with the EFN solution indicated by the dotted line. The unbroken curve is based on values obtained by a computer calculation [98] and gives the exact solution of the above formula. The excellent agreement proves that the stress field of dislocations had been predicted correctly by the theory.

\subsection{Dislocation Networks and Dislocation Interactions}

The polygonization wall discussed in the previous section is only one special case of subboundaries. In general, they consist of dislocation networks of fair regularity. In 1953 such networks had already been observed by Hedges and Mitche11 [6], and their theoretical interpretation was given by Frank [99]. Subsequently, other investigators, particularly Amelinckx and Dekeyser [100], made detailed comparisons between theoretical predictions and experiments. They have explored ionic crystals experimentally in great detail and have been able to explain networks, including the occasional irregularities in networks.

Among other results, such investigations have provided positive proof of the identity of the Burgers vectors found with those predicted and also for the occurrence of predicted dislocation reactions. In particular, hexagonal networks can be formed from two sets of straight intersecting dislocations with different Burgers vectors, through their reaction at the nodes.

Figures 1, 3, and 30 give some examples of dislocation networks in silver chloride, sodium chloride and bismuth telluride. 
An extensive study of dislocation interactions in stainless steel has been carried out by Whelan [101], and Mitchell and his co-workers found evidence for a number of dislocation interactions in ionic crystals (see references in 1.2).

\subsection{Cross-Slip and Dislocation "Trails" in Thin Foils}

Unextended screw dislocations in isotropic materials have radial symmetry and can, in principle, move in any plane containing their axis. In actual fact, the choice of slip planes, even for screw dislocations, is more restricted. Nonetheless, since the discovery of slip lines on the so-called "cross-slip" system by Maddin, Mathewson, and Hibbard [102] in $\alpha$-brass and Cahn's investigations of similar nature in aluminum [103], it is recognized that screw dislocations may abruptly move from one slip plane into another one which is inclined to the first. This process is called cross-slip, and many micrographs are now available demonstrating it in electron transparent foils $[1,81,104]$.

One such example is given in figure 31 in which the motion executed by the dislocation before reaching its final position is clearly marked by a trail. The nature of such trails, very prevalent in thin foils, is still open to dispute. Depending on the material of the foil, or possibly its surface film, the trails are stronger or weaker and "bleach" out after shorter or longer periods of time, remaining visible up to a few minutes.

\subsection{Dislocation Velocities}

While moving dislocations can only be seen directly in the electron microscope, thin film techniques do not lend themselves easily to quantitative measurements of the dependence of dislocation velocity on stress. The reason for this is mainly that it is very difficult to determine the stresses in thin foils. On the other hand, repeated etching experiments have been successfully applied to different materials. The results obtained so far are most intriguing, since in some crystals with diamond lattices, the dislocation velocity $\mathrm{v}$ rises only as $\mathrm{v} \propto \tau$ with $\mathrm{n}=1.5$, while in some ionic crystals and metals $n \approx 25$ and higher. Figures 32, 33, and 34 show the measurements obtained on lithium fluoride [105], silicon-iron [106], and germanium [107].

\subsection{Dislocation Densities}

On first thought one would expect that the measurement of dislocation densities by diffraction electron microscopy provides more accurate data than any other technique.

Indeed, the approach is simple and direct as was shown recently [108]. Measuring $R_{p}$, the total projected length of dislocation line in an area $A$, the dislocation density $\rho$ is found as $p=(4 / \pi) R_{p}$, $t$ being the film thickness. However, the measurement of $R_{p}$ is time consuming and may be replaced by counting the number of intersections $N$ of dislocations with a random set of Iines with a total length L [109, I10]. With $R_{0}=\pi N A / 2 L$, the density $\rho=2 \mathrm{~N} / \mathrm{Lt}$. This method works best in the range from $10^{8}$ to $\mathrm{P}_{10} 0^{10}$ dislocations per $\mathrm{cm}^{2}$, since $\mathrm{N}$ has to be large (about 50). The practical limitations of the method are the following: For high densities $R$ is determined with a considerable error, because in foils with $t>2 \times 10^{-5} \mathrm{~cm}$ (as is required [86]), the overlap of dislocation images becomes rather serious; this will be the case for dislocation densities $p \geq 10^{10} \mathrm{~cm}^{-2}$. On the other hand, for $t<10^{-6} \mathrm{~cm}$, $\rho$ is reduced by dislocations leaving through the surface $[111,86]$ and because of rearrangements within the foil. Also, the magnification required for these measurements is $20,000 \mathrm{x}$ and higher so that only small areas become available for measurement. Obviously, many micrographs have to be taken in order to obtain a representative cross section of the crystal's dislocation density. In addition to these shortcomings, it should be kept in mind that the specimen preparation for diffraction electron microscopy is a destructive one.

The latter difficulty is not inherent in X-ray techniques (see 1.4.a) which offer, in addition, the easy examination of large areas and, therefore, a time saving coupled with higher accuracy. However, the drawback of X-ray methods is that their optimum application is in the range of $\rho \cong 10^{5} \mathrm{~cm}^{-2}$ which is rather low for most practical cases. It thus becomes apparent that thin film and X-ray techniques for the determination of dislocation densities have severe drawbacks, so that for many applications the extra experimental effort is not justified, and etch pit techniques are used instead wherever feasible. 
Finally, the distribution of dislocations is often far from random. A portion of the dislocations is usually concentrated in networks or other dislocation arrays of high density, a feature which complicates dislocation counts rather seriously (see also the review article by Hirsch [112]).

\subsection{Dislocation Climb}

Climb, the motion of dislocations normal to their slip planes, takes place when dislocations either absorb or emit vacancies or interstitials. In the case of metals, because of the high energy of interstitials, it is primarily vacancy absorption and emission which causes climb.

The process of polygonization in itself is powerful evidence for climb. More recently, climb has been observed directly in the electron microscope and was described in a number of papers $[113,114,115,116]$.

\subsection{The Origin of Dislocations}

\section{a. Grown-in Networks}

In the process of crystallization, subboundaries are formed which consist of dislocation arrays. In a recent study, Damiano has followed the various stages of subboundarles in zinc [46], beginning with their generation during solidification, through the cooling process, until (under suitable conditions) they apparently disappeared.

The subboundaries observed by Mitchell and coworkers and Amelinckx and coworkers were formed during recrystallization and cooling [100]. Random dislocations are also formed during crystallization, many of them originating at irregular sites in the mold material. Lang has been able to obtain stereo-topographs of dislocations in as-grown crystals of various materials with his X-ray technique [75]; these photographs give a most vivid impression of the arrangements of in-grown dislocations, partly forming subboundaries, partly arranged at random.

\section{b. Frank-Read Sources}

On applying a stress in the plastic region, the dislocation content of crystals is known to rise dramatically. Just how the requisite dislocation multiplication could take place was a mystery until Frank and Read proposed their source mechanism [117].

The first direct evidence obtained for this mechanism in a metal is represented in figure 5 , in which concentric decorated dislocations are seen by means of a replica technique. Only arcs are visible on the micrograph because the plane of the replicated surface is slightly inclined against the active slip plane. Since then, decorated dislocations arranged in patterns expected from the Frank-Read mechanism have been found repeatedly $[36]$. A beautiful example in silicon is shown in figure 35 [24].

The action of such sources was finally observed and photographed in thin foils of stainless steel [118] (figures 36 and 37 ).

\section{c. Dislocations Released from Subboundaries}

Dislocation links, acting as Frank-Read sources, may be identical with dislocation links in subboundaries. However, although this may be true occasionally, it seems to be more common that dislocations in subboundaries leave these altogether on application of a stress, so that the subboundaries dissolve gradually. The sequence of micrographs in figure 38 shows this phenomenon [81]. It is also substantiated by the observation that subboundaries seem to disappear after smal1 plastic strains [33] and that thin foils obtained from lightly deformed crystals do not show any dislocation networks of the type found in as-grown specimens.

\section{d. Dislocations in Interfaces}

As was first pointed out by van der Merwe and Frank [119], the misfit which exists between crystals of two different materials adjoining on a plane with almost matching atomic configurations (as for example happens in oriented overgrowth) is expected to be concentrated in the cores of spontaneously formed dislocations. The underlying principle is the same as for the formation of dislocation networks where slightly misoriented crystals meet (i.e., subboundaries).

Dislocations which apparently are formed in this way have now been observed on chromium bromide platelets by diffraction electron microscopy [120]. 
e. Spontaneous Generation of Dislocations at Stress Peaks

If, locally, a stress arises which is larger than the theoretical stress that an ideal crystal could support, then dislocations may be generated spontaneously.

Striking examples of this phenomenon, resulting from stresses associated with precipitates, were found in crystals by Jones and Mitchel1 [16], and others have been reported by various authors $[121,122]$. Figure 39 shows this spontaneous generation of dislocations in the form of sequences of loops originating at the site of a precipitate in beryllium.

Similarly, when fine hard particles are sprinkled onto ionic crystals [58] and metals [60], "rosettes" of pits are produced by subsequently etching the surface (figure 40). At least a fraction of such rosettes seem to be due to spontaneous generation of dislocations.

\section{f. Dislocations Formed by the Condensation of Thermal Vacancies}

When thermal vacancies condense in the form of a continuous layer on a closepacked crystal plane, the sides of the missing layer of atoms will collapse, once its area has exceeded a critical size. The rim of the area in which one layer of atoms is missing constitutes a prismatic dislocation loop. Prismatic loops of this kind were actually observed in some rapidly quenched metals after fairly detailed predictions had been made regarding their formation [123]. Gratifyingly, all predictions have since been found correct $[124,125]$ with the exception of the size of the loops, which is smaller than had been expected. Figure 41 shows the discussed kind of "quench loops" in aluminum.

One prediction regarding quench loops was that they should act as dislocation sources when of sufficient size, or would emit dislocation loops when other glide dislocations passed near them [123]. This, too, has since been observed [115], as can be seen from figure 42 .

Vacancies in supersaturation do not always condense in the form of isolated prismatic loops, but often condense in contact with existing dislocations. However, while earlier theory [126] had predicted a continuous climb of dislocations resulting from such vacancy condensation, the direct experimental results usually show prismatic loops in contact with already existing dislocations [115].

A third mode of vacancy condensation is observed in connection with screw dislocations. In several quenched fcc alloys and in silicon, screw dislocations are found to climb into long, rather regular helices when vacancies condense on them $[15,127$, $128,26]$.

\section{g. Dislocation Multiplication in Slip Bands}

As first shown by Johnston and Gilman [129], even a single, isolated loop of a glide dislocation expanding on its own slip plane under the action of an applied stress can multiply and give rise to a densely populated glide band. Figure 43 illustrates this phenomenon in LiF. We will limit ourselves to giving the experimental evidence since the details of the mechanisms involved are still under dispute.

\subsection{Evidence on the Burgers Vector of Imperfect Dislocations}

Whenever atomic positions of relative energy minimum exist on a slip plane, as, for example, the so-called stacking fault positions on $\{111\}$ planes in fcc crystals, imperfect dislocations are theoretically possible. Imperfect dislocations are those dislocations which must exist at the boundaries between faulted regions and perfect regions, as well as at the boundaries of regions faulted in different ways. From a theoretical standpoint such imperfect dislocations and their possible Burgers vectors were examined long before it became possible to observe them in the electron microscope $[130]$.

The most simple kind of imperfect dislocations are the partials into which a perfect glide dislocation in a fcc crystal may split to form an extended dislocation. Their existence is now established beyond any doubt through diffraction electron microscopy ( see 1.4.b, figure 20). As can be seen, the stacking fault ribbons in extended dislocations appear clearly as striae when viewed with the electron microscope under suitable diffraction conditions. 
The existence of another kind of imperfect dislocation, the so-called simple stair rod dislocation, has also been observed. It arises at the lines of intersection between stacking faults on different planes. Examples of stair rod dislocations are found in evaporated films [131], and, moreover, they form the edges of the stacking fault tetrahedra into which quench loops may transform [132].

The study of imperfect dislocations is particularly rewarding in layer structures. Thin foils are readily prepared from them by cleavage. Furthermore, the orientation of the plane of the foil is always parallel to the preferred crystal plane which carries the dislocations, and stacking fault energies are low so that the equilibrium widths of the stacking faults are large. Amelinckx and Delavignette have made careful studies of dislocation configurations in layer crystals and were able to account for the bulk of the observations $[133,134]$.

\subsection{Dislocation Tangling and Long Prismatic Loops}

Almost all phenomena discussed up to this point had been theoretically derived or had been predicted on the basis of indirect experimental evidence. Only one very striking major phenomenon is observed extensively, the existence of which had not been anticipated. This is "dislocation tangling." Electron transparent foils, obtained from previously deformed specimens of pure metals, do not exhibit the orderly sequences of smooth dislocation lines as anticipated, but instead are filled with tangles of irregularly kinked and intertwined dislocations, interspersed with tiny prismatic dislocation loops [135]. Figure 44 demonstrates that a tangled group of dislocations is three-dimensional in character, which is an important characteristic. Dislocation tangles are found in aluminum after elongations of 1 percent or even less [86]. This is in sharp contrast to dislocation structures arising in foils when strained while under observation in the electron microscope. In the latter case the dislocations remain straight $[87,104]$, even when they are oriented almost parallel to the foil surface so that they are quite long [136]. Tangles are in contrast with dislocation configurations observed in $70 / 30$ brass (figure 45), ordered and disordered $\mathrm{Cu}_{3} \mathrm{Au}$ (figures 46 and 47), all of which were deformed as crystals thicker than $1 \mathrm{~mm}[137,138]$.

Although there is not as yet a clear understanding of the origin of the tangles, much evidence has been accumulated which indicates that they are due to dislocationpoint defect interactions [139].

Another unexpected feature of dislocations due to deformation is that they often form long, narrow prismatic loops $[136,140,141]$ trailing behind screw dislocations, as shown in figure 48. Also their origin is still under dispute, although various theories have been proposed.

\subsection{Radiation Damage}

Point defects cannot directly be observed by any of the techniques discussed in detail in section 1 . However, if vacancies agglomerate and condense, their detection is indirectly possible as shown in 2.9.f. In studies of radiation damage by pile neutrons, a number of metals have been examined by etch pit techniques [142] and by diffraction electron microscopy $[143,144]$. Often, irradiated metals exhibit small dislocation loops of about $200 \AA$ in diameter, as well as unresolved dark spots whose diameter is less than $100 \AA$ or even $50 \AA$. In other cases, irradiated, undeformed metals such as nickel or stainless steel do not contain any type of defect which could be observed by means of electron microscopy $[145,146]$. Subsequent deformation produces in nickel dislocation tangles which contain dislocations with very short link length and many small prismatic dislocation loops. It is remarkable that the passage of glide dislocations through irradiated specimens removes visible evidence of radiation damage [144].

I have greatly profited from many discussions with my wife, Dr. Doris Wilsdorf, University of Pennsylvania, Philadelphia, whom I thank for her contribution.

This paper was sponsored in part by the Aeronautical Research Laboratories, Office of Aerospace Research, USAF, under Contract No. AF 33(616) -6996 .

\section{References}

[1] P. B. Hirsch, R. W. Horne, and M. J. Whelan, Phil. Mag. 1, $677^{\text {(1956); }}$ movie on dislocations in aluminum, followed by another on dislocations in stainless steel.
[2] F. W. C. Boswell and F. Weinberg, Dislocation Movements in $\alpha=$ Brass, 1958. 
[3] A. H. Cottre11, Report on Strength of Solids, p. 30 (London, Phys. Soc., 1948).

[4] C. Crussard, Métaux et Corrosion 25, 301,203 (1950).

[5] H. Suzuki, Rep. Tohoku Univ. Res. Inst. A4, 455 (1952).

[6] J. M. Hedges and J. W. Mitchell, Phil. Mag. 44, 223 (1953).

[7] S. Amelinckx, Phil. Mag. 1, 269 (1956).

[8] P. L. Pratt, Rep. Conf. on Defects in Cryst. Solids. p. 402 (London, Phys. Soc., 1955).

[9] W. van der Vorst and W. Dekeyser, Phil. Mag. 1, 882 (1956).

[10] D. J. Barber, K. B. Harvey, and J. W. Mitche11, Phil. Mag. 2, 704 (1957).

[11] J. T. Bartlett and J.W. Mitchell, Phi1. Mag. 3 , 334 (1958).

[12] W. Bontinck and W. Dekeyser, Acta. Met. 7., 389 (1959).

[13] W. Bontinck and W. Dekeyser, Physica, 22, 595 (1956).

[14] S. Amelinckx, Phil. Mag. 3, 307 (1958).

[15] W. Bontinck and S. Amelinckx, Phil. Mag. 2, 94 (1957).

[16] D. A. Jones and J. W. Mitchell, Phil. Mag. 3,1 (1958).

[17] S. Amelinckx, Phil. Mag. $\underline{3}, 653$ (1958).

[18] A. S. Parasnis and J. W. Mitche11, Phil. Mag. 4, 171 (1959).

[19] J. T. Bartlett and J. W. Mitchell, Phil. Mag. 5, 445 (1960).

[20] J. T. Bartlett and J. W. Mitchell, Phil. Mag. $\underline{5}, 799$ (1960).

[21] J. T. Bartlett and J. W. Mitche11, Phi1. Mag. $\underline{6}, 271$ (1961).

[22] C. B. Childs and L. Slifkin, Phys. Rev. Letters, $\underline{5}, 502$ (1960).

[23] R. J. Haynes and W. Shockley, Phys. Rev. 82, 935 (1951).

[24] W. C. Dash, J. App1. Phys. 27, 1193 (1956); Dislocations and Mechanical Properties of Crystals, p. 62, John Wiley \& Sons (New York, 1957).

[25] W. C. Dash, J. Appl. Phys. 29, 736 (1958).

[26] W. C. Dash, J. App1. Phys. 31, 2275 (1960).
[27] W. L. Fink and D. W. Smith, Trans. AIME, 122, 284 (1936); Ibid. 128, 223 (1938).

[28] G. Wassermann, Z. Metallkd, 30, 62 (1938).

[29] M. L. V. Gayler, J. Inst. Metals, 66, 72 (1940).

[30] R. Castaing and A. Guinier, Compt. Rend. 228, 2033 (1949).

[31] P. Lacombe and A. Berghezan, Compt. Rend. 228, 365 (1949).

[32] H. Wilsdorf and D. Kuhlmann-Wilsdorf, Phil. Mag. 45, 1096 (1954).

[33] H. Wilsdorf and D. Kuhlmann-Wilsdorf, Rep. Conf. on Defects in Cryst. Solids, p. 175 (London, Phys. Soc., 1955).

[34] G. Thomas and J. Nutting, Inst. Metals Symp. "Mechanism of Phase Transf. In Metals," p. 57 (1955).

[35] T. Takeyama and S. Koda, Nature (London) 179,777 (1957).

[36] P. A. Jaquet and A. R. Weill, Rev. Met. 58, 97 (1961).

C. G. Dunn and W. R. Hibbard, Acta Met. $\underline{3}, 409$ (1955).

[38] W. R. Hibbard and C. G. Dunn, Acta Met. 4,306 (1956).

[39] J. C. Suits and J. R. Low, Acta Met. $\underline{5}, 285$ (1957).

[40] J. R. Low and R. W. Guard, Acta Met. 7., 171 (1959).

[41] R. Sun and H. Wilsdorf, J. Franklin Inst. 265,413 (1958).

[42] J. D. Meakin and H. G. F. Wilsdorf, Trans. AIME 218, 737, 745 (1960).

[43] J. J. Gilman, Trans. AIME 206, 998 (1956).

[44] V. V. Damiano and M. Herman, Trans. AIME 215,136 (1959).

[45] V. V. Damiano and G. S. Tint, Acta. Met. 9,177 (1961).

[46] V. V. Damiano, Ph.D. Thesis, Univ. Pennsylvania, 1961 .

[47] G. S. Tint and V. V. Damiano, Rev. Sci. Instr. 32, 325 (1961).

[48] J. J. Gilman, The Surface Chemistry of Metals and Semiconductors, p.136, John Willey \& Sons (New York, 1960 ). 
[49] W. Kossel, Nachr. Ges. Wiss. Gottingen, [71] 1927 , p. 135 .

[50] W. G. Johnston, Progress in Ceramic Science, Pergamon Press, to be published. G. E. Research Lab. Rept. No. 6I-RL-2649M, 1961 .

[51] J. J. Gilman, W. G. Johnston, and G. W. Sears, J. Appl. Phys. 29, 747 (1958).

[52] M. B. Ives and J. P. Hirth, J. Chem. Phys. 33, 517 (1960).

[53] W. Riessler, Z. angew. Phys. 12, 433 (1960).

[54] N. Cabrera and M. M. Levine, Phil. Mag. 1,450 (1956).

[55] N. Cabrera, Semiconductor Surface Physics, p. 327, Univ. of Pennsylvania Press (1957).

[56] N. Cabrera, The Surface Chemistry of Metals and semiconductors, p. 71 , John Wiley \& Sons (New York, 1960).

[57] S. Amelinckx, Acta Met. 2, 848 (1954).

[58] J. J. Gilman and W. G. Johnston, Dislocations and Mechanical Properties of Crystals, p. 116, John Wiley \& Sons (New York, 1959).

[59] R. J. Stokes, T. L. Johnston, and C. H. Li, Trans. AIME, 215, 437 (1959).

[60] J. D. Livingston, G. E. Research Lab. Rept. No. 61-RL-2620M, 1961.

[61] J. D. Livingston, J. App1. Phys. 31, 1071 (1960).

[62] F. W. Young, J. Appl. Phys. 32, 1815 (1961).

[63] V. V. Damiano and M. Herman, J. Frank1in Inst. 267, 303 (1959).

[64] V. R. Regel, A. A. Urusovskaya, and V. N. Kolomiichuk, Kristallografiya, 4, 937 (1959).

[65] S. Kitajima, J. Inst. Metals (Japan) 18, 592 (1954).

[66] H. Suzuki, J. Phys. Soc. (Japan) 10, 981 (1955).

[67] A. A. Hendrickson and E. S. Machlin, Acta. Met. $\underline{3}, 64$ (1955).

[68] J. P. Hirth and L. Vassamillet, J. App1. Phys. 29, 595 (1958).

[69] E. Votava and A. Berghezan, Acta. Met. 7., 392 (1959).

[70] G. K. Wehner, Adv. in Electronics and Ei. Phys. $\underline{7}, 239$ (1955).
G. K. Wehner, J. App1. Phys. 29, 217 (1958).

[72] B. B. Meckel and R. A. Swalin, J. App1. Phys. 30, 89 (1959).

[73] P. E. Doherty and R. S. Davis, Acta Met. I, 118 (1959).

[74] A. R. Lang, J. Appl. Phys. 29, 597 (1958).

[75] A. R. Lang, J. App1. Phys. 30, 1748 (1959).

[76] J. B. Newkirk, Trans. AIME, 215, 483 (1959).

[77] J. M. Lommel, private communication.

[78] W. Bollmann, Phys. Rev. 103, 1588 (1956).

[79] G. Thomas, Transmission Electron Microscopy of Metals, John Wiley \& Sons (New York, 1962).

[80] P. R. Strutt, Rev. Sci. Instr. $\underline{32}$, 411 (1961).

[81] H. G. F. Wilsdorf, ASTM Spec. Techn. Pub1. No. 245, p. 43 (1958).

[82] H. G. F. Wilsdorf, Rev. Sci, Instr. 29,323 (1958); H. G. F. Wilsdorf, L. Cinquina, and C. J. Varker, Proc. 4th Intern. Conf. Electron Microscopy, p. 559, Springer (Berlin, 1960).

[83] B. H. Kear, Rev. Sci. Instr. 31, 1007 (1960).

[84] J. T. Fourie, F. Weinberg, and F. W. C. Boswell, Twin Formation in Tin Foils $(1959)$.

[85] J. S. Koehler, Phys. Rev. 86, 52 (1952).

[86] H. G. F. Wilsdorf and J. Schmitz, J. Appl. Phys. 33 (May, 1962).

[87] G. Dupouy and F. Perrier, Compt. Rend. 253, 2435 (1961).

[88] W. L. Bond and J. Andrus, Phys. Rev. 101, 1211 (1956).

[89] J. W. Menter, Proc. Roy. Soc. A236, 119 (1956).

[90] G. A. Bassett, J. W. Menter, and D. W. Pashley, Proc. Roy. Soc. A246, 345 (1958).

[91] E. W. Muller, J. Appl. Phys. 30, 1843 (1959).

[92] F. L. Vogel, W. G. Pfann, H. E. Corey, and E. E. Thomas, Phys. Rev. 90, 489 (1953). 
[93] J. M. Burgers, Proc. Phys. Soc. (London) $\underline{52}, 23$ (1940).

[94] F. L. Voge1, Acta Met. 3 , 245 (1955).

[95] H. G. F. Wilsdorf, unpublished.

[96] R. W. Cahn, J. Inst. Metals 76, 121 (1949).

[97] J. D. Eshelby, F. C. Frank, and F. R. N. Nabarro, Phil. Mag. 42, 351 (1951).

[98] D. Kuhlmann-Wilsdorf, private communication.

[99] F. C. Frank, Rept. Conf. on Defects in Cryst. Solids, p. 159 (London, Phys.

[100] See the extensive review article by S. Amelinckx and W. Dekeyser, Solid State Physics, 8, p. 325, Acad. Press (New York, 1959).

[101] M. J. Whelan, Proc. Roy. Soc. A249, 114 (1958).

[102] R. Maddin, C. H. Mathewson, and W. R. Hibbard, Trans. AIME, $\underline{175}, 86$ (1948).

[103] R. W. Cahn, J. Inst. Metals 79, 129 (1951).

[104] A. Berghezan and Fourdeux, J. App1. Phys. 30, 1913 (1959).

[105] W. G. Johnston and J. J. Gilman, J. App1. Phys. 30, 129 (1959).

[106] D. F. Stein and J. R. Low, J. App1 . Phys. 31, 362 (1960).

[107] A. R. Chaudhuri, J. R. Pate1, and

L. G. Rubin, J. App1. Phys. 33 (1962).

[108] J. E. Bailey and P. B. Hirsch, Phil. Mag. 5,485 (1960).

[109] R. K. Ham, Phil. Mag. 6, 1183 (1961).

[110] C. S. Smith and L. Guttman, J. Metals 5, 87 (1953).

[111] R. K. Ham and N. G. Sharpe, Phil. Mag. 6, 1193 (1961).

[112] P. B. Hirsch, Progr. in Metal Physics 6, $235(1956)$.

[113] J. Silcox and M. J. Whelan, Phil. Mag. $\underline{5}, 1$ (1960).

[114] F. Kroupa and P. B. Price, Phil. Mag. $\underline{6}, 243$ (1961).

[115] D. Kuhlmann-Wilsdorf, R. Maddin, and H. G. F. Wilsdorf, ASM Seminar "Strenthening Mechanisms in Solids"(Philadelphia, 1960).
[116] P. B. Price, Proc. Intern. Conf. Transmission Electron Microscopy, in the press (Berkeley, California, 1961).

[117] F. C. Frank and W. T. Read, Phys. Rev. 18,722 (1950).

[118] H. G. F. Wilsdorf, Proc. Intern. Conf. Structure and Properties of Thin Films, p. 151, John Wiley \& Sons (New York, 1959).

[119] F. C. Frank and J. H. van der Merwe, Proc. Roy. Soc. A198, 205 (1959); Ibid. 200, 125 (1950); Ibid. 201, 261 (1950).

[120] P. Delavignette, J. Tournier, and S. Amelinckx, Phil. Mag. $\underline{6}, 1419$ (1961).

[121] A. Fourdeux and A. Berghezan, Compt. Rend. 252, 1462 (1961).

[122] F. Wilhelm, private communication.

[123] D. Kuhlmann-Wilsdorf, Phil. Mag. 3. 125 (1958).

[124] P. B. Hirsch, J. Silcox, R. E. Sma11man, and $\mathrm{K}$. H. Westmacott, Phil. Mag. $\underline{3}, 897$ (1958).

[125] D. Kuhlmann-Wilsdorf and H. G. F. Wilsdorf, J. App1. Phys. 31, 516 (1960).

[126] J. Bardeen and C. Herring, Imperfections in Nearly Perfect Crystals, John Wiley \& Sons, p. 261 (New York, 1952).

[127] S. Amelinckx, W. Bontinck, W. Dekeyser, and F. Seitz, Phil. Mag. 2, 355 (1957).

[128] G. Thomas and M. J. Whelan, Phil. Mag. 4, 511 (1959).

[129] W. G. Johnston and J. J. Gilman, J. App1. Phys. 31, 632 (1960).

[130] F. C. Frank and J. F. Nicholas, Phil. Mag. 44, 1213 (1953).

[131] J. W. Matthews, Phil. Mag. 4, 1017 (1959).

[132] J. Silcox and P. B. Hirsch, Phil. Mag. 4, 72 (1959); R. E. Smallman and K. H. Westmacott, J. Appl. Phys. 30, 603 (1959).

[133] S. Amelinckx and P. Delavignette,

J. App1. Phys. 32, 341 (1961).

[134] S. Amelinckx and P. Delavignette, Phil. Mag. $\underline{5}, 729$ (1960). 
[135] H. G. F. Wilsdorf and D. KuhlmannWilsdorf, Phys. Rev. Letters $\underline{3}, 170$ (1959).

[136] J. T. Fourie and H. G. F. Wilsdorf, J. App1. Phys. 31, 2219 (1960).

[137] P. R. Strutt and H. G. F. Wilsdorf, to be published.

[138] B. H. Kear and H. G. F. Wilsdorf, Trans. AIME 224 (1962), in press.

[139] D. Kuhlmann-Wilsdorf and H. G. F. Wilsdorf, Proc. Intern. Conf. Transmission Electron Microscopy, Berkeley, Calif., 1961, in press.

[140] J. Washburn, G. W. Groves, A. Kelly, and G. K. Williamson, Phil. Mag. 5, 991 (1960).

[141] P. B. Price, J. App1. Phys. 32, 1750 (1961).

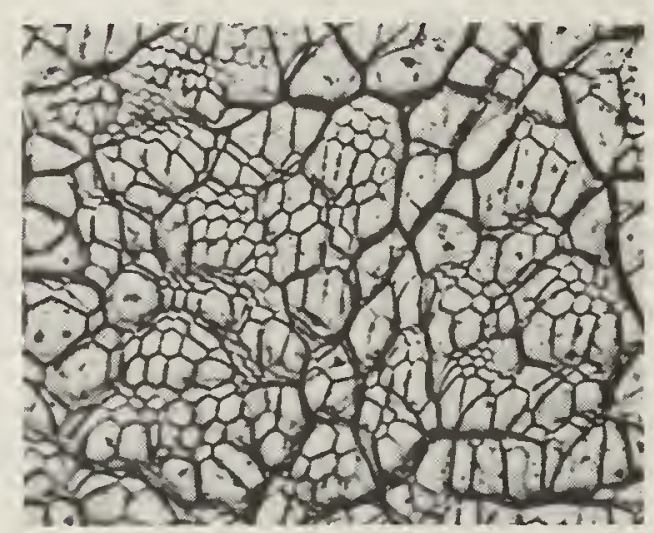

FIGURE 1. Dislocations in subboundaries after decoration with photolytic silver in $\mathrm{AgBr}(1125 \mathrm{X})$. (courtesy of J.W. Mitchell and D. A. Jones.)
[142] J. J. Gilman and W. G. Johnston, J. App1. Phys. 29, 877 (1958).

[143] J. Silcox and P. B. Hirsch, Phil. Mag. 4, 1356 (1959).

[144] I. G. Greenfield and H. G. F. Wilsdorf, Naturwissensch. $\frac{47}{32}, 395$ (1960); J. App1. Phys. $\underline{32}, 827$ (1961).

[145] H. G. F. Wilsdorf, Phys. Rev. Letters 3 , 172 (1959).

[146] H. G. F. Wilsdorf and D. KuhlmannWilsdorf, J. Nuc1. Materials, in press.

[147] W. T. Read, Dislocations in Crystals, McGraw Hill (New York, 1953).

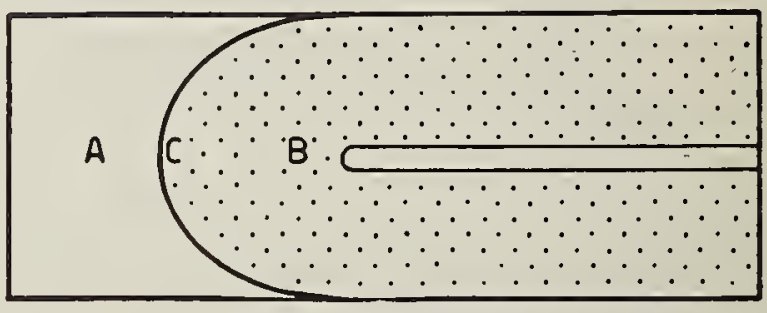

FIGURE 2. Drawing to illustrate an experimental technique for decorating dislocations in $\mathrm{NaCl}$. (courtesy of S. Amelinckx [7].)

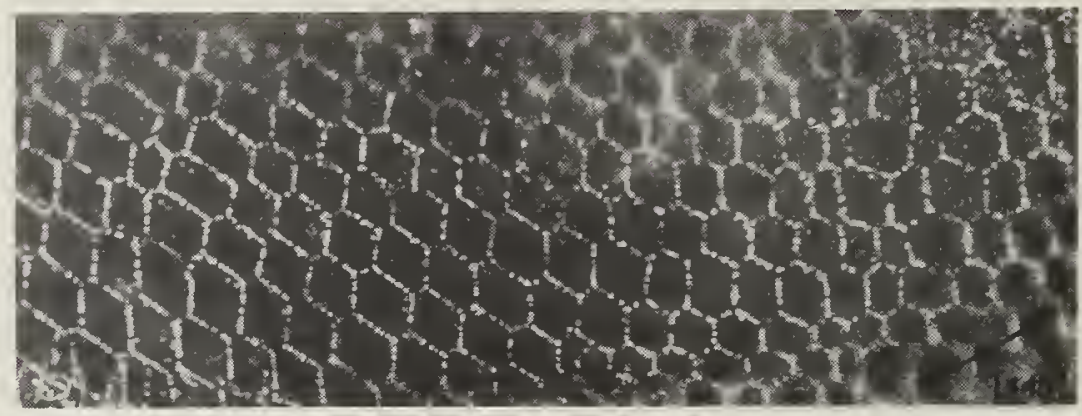

FIGURE 3. Hexagonal network of dislocations in $\mathrm{NaCl}(450 \mathrm{X})$.

(courtesy of S. Amelinckx, Acta Met. 6, 34 1958.) 


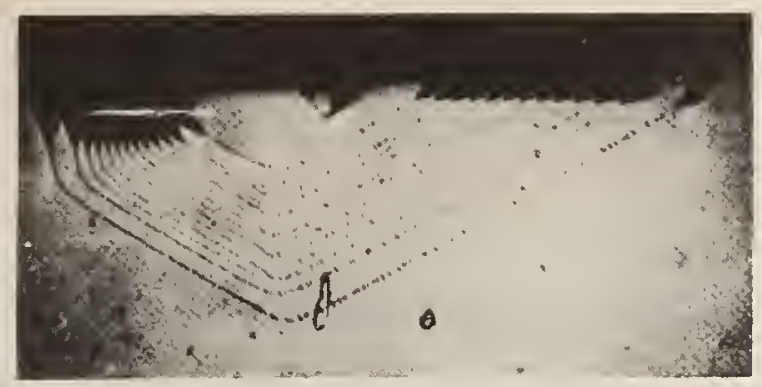

FIGURE 4. Dislocations in silicon ending at the surface which was etched. Decoration was achieved by the diffusion of copper. Photographic technique used infrared light.

(courtesy of W. C. Dash [24].)

FIGURE 5. Decorated dislocation loops in $\mathrm{A} 1-2 \% \mathrm{Cu}$; since surface is somewhat inclined against (111) plane, only segments of dislocations are seen. Direct silicon monoxide replica ( $15,000 \mathrm{X}$ [32]).
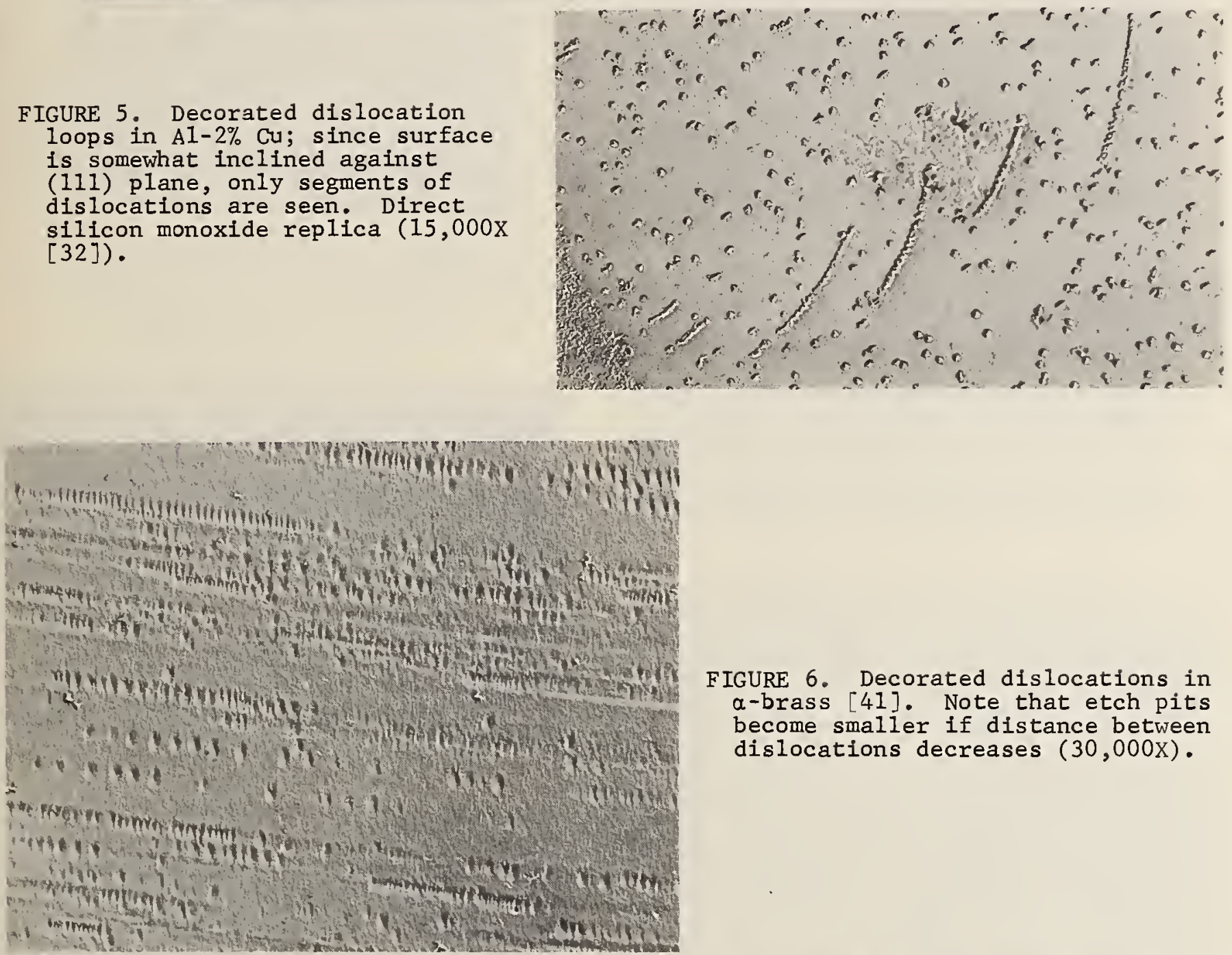

FIGURE 6. Decorated dislocations in $a$-brass [41]. Note that etch pits become smaller if distance between dislocations decreases $(30,000 \mathrm{X})$.

FIGURE 7. Dislocation subboundaries in as-grown zinc. Single dislocations are not resolved. Basal plane parallel to growth direction $(30 \mathrm{X})$.

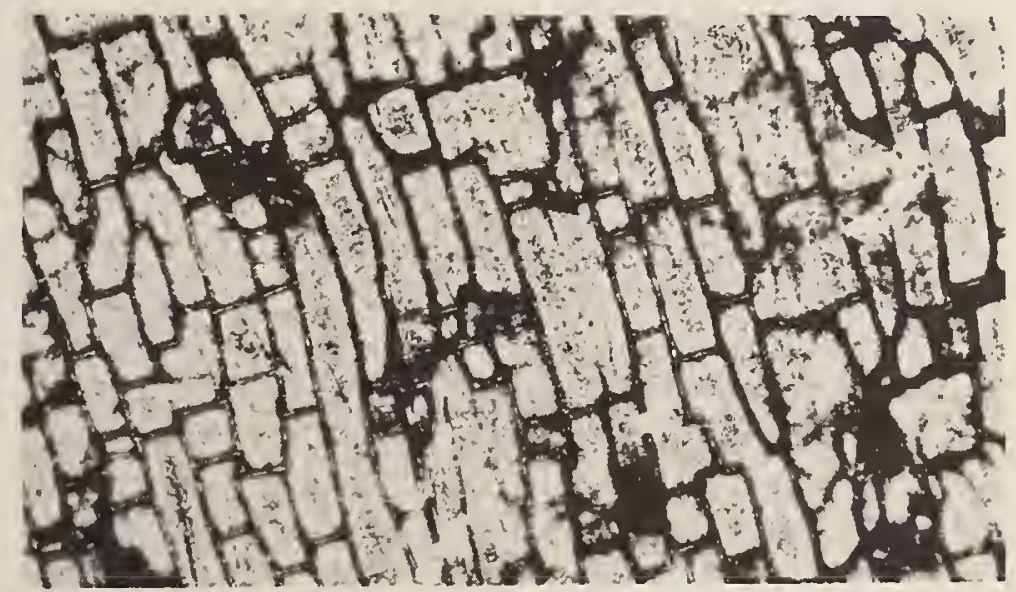




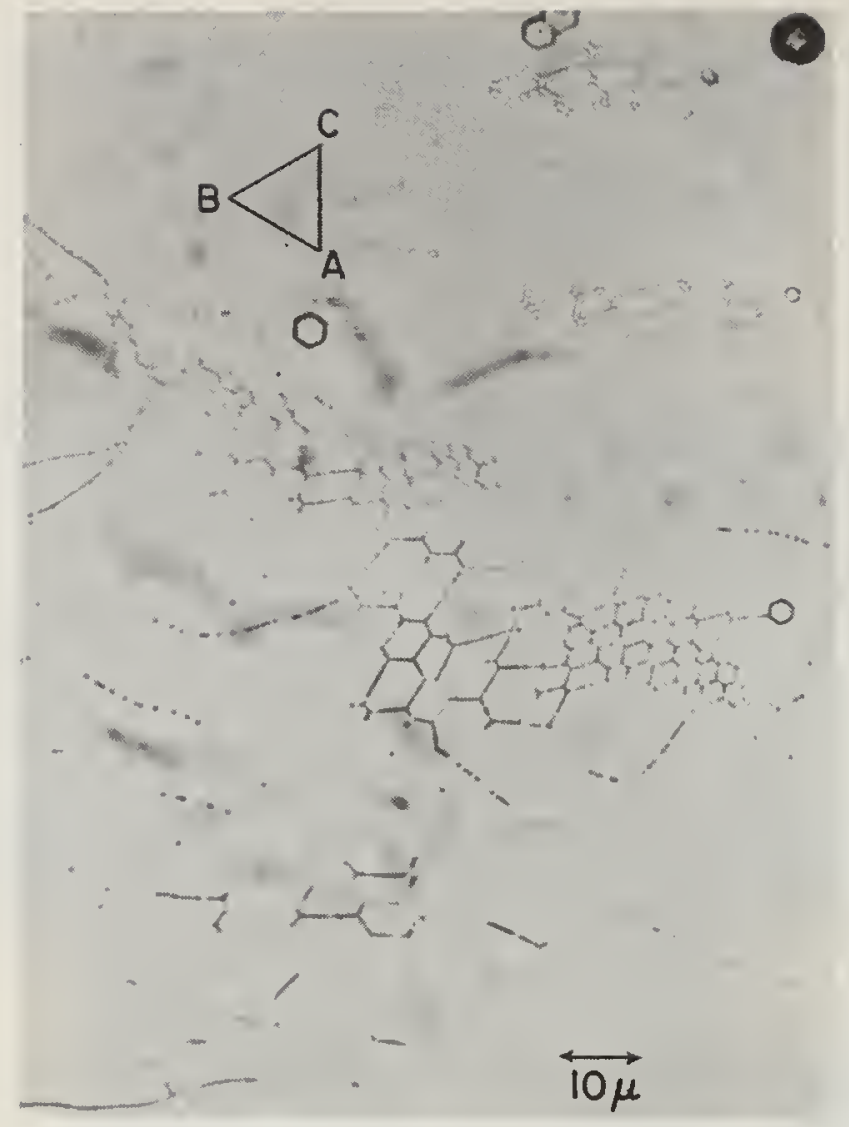

FIGURE 8. Decorated dislocations in (0001) plane of as-grown zinc crystal. <1120> directions are shown.

(courtesy of V. V. Damiano [46].)
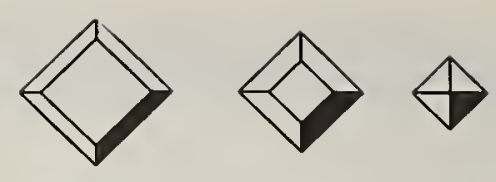

Initial
dislocation

position

Fìnal dislocation position

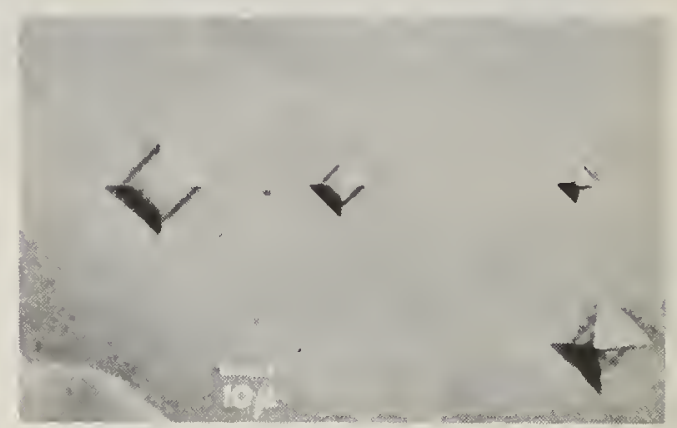

FIGURE 10. Effect of etching on shape of pits after moving the dislocation.

(courtesy of J. J. Gilman and .W. G. Johnston [58].)

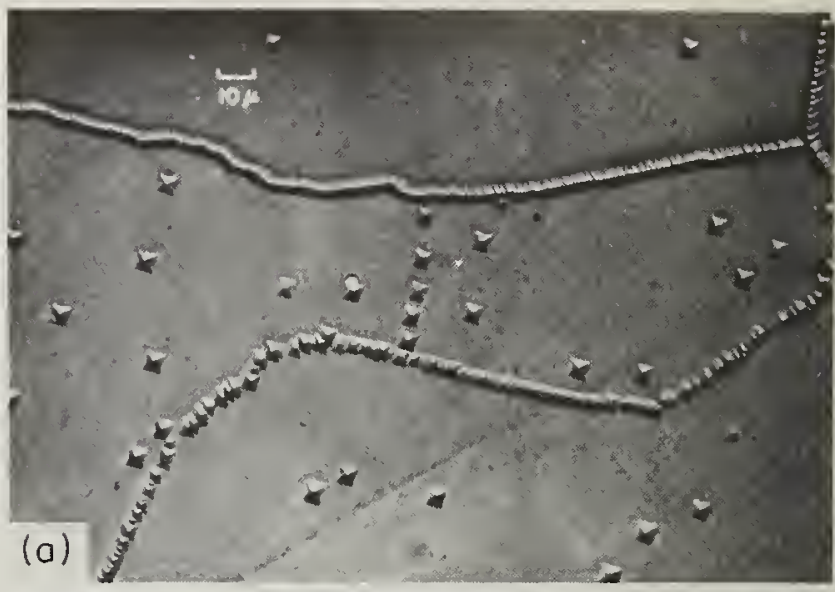

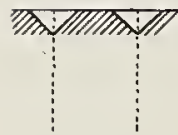
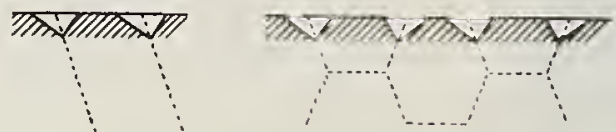

$\bigotimes 凶$

$\triangle \bigotimes$

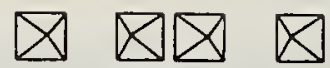

$\oplus \diamond$

(a)

\section{$\triangleq \triangleq$}

(b)

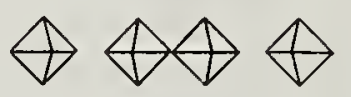

(c)
FIGURE 9. Illustration of connection between shape of etch pits and angle under which dislocations meet the surface.

(courtesy of S. Amelinckx [7].)

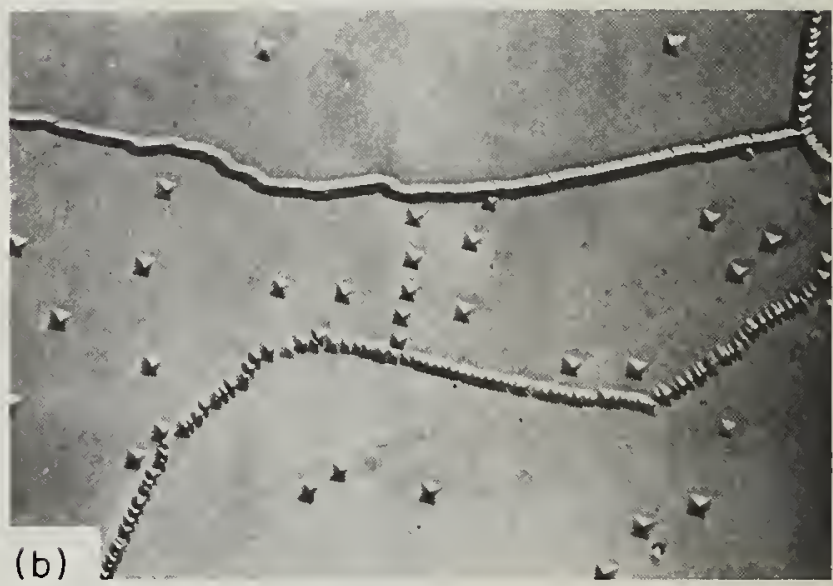

FIGURE 11. Identical etch pits on cleavage faces of LiF provide proof that $1: 1$ ratio between dislocations and pits can be obtained ( $a$ and $b$ represent matching faces of the cleaved crystal). (courtesy of J. J. Gilman and W. G. Johnston [58].) 


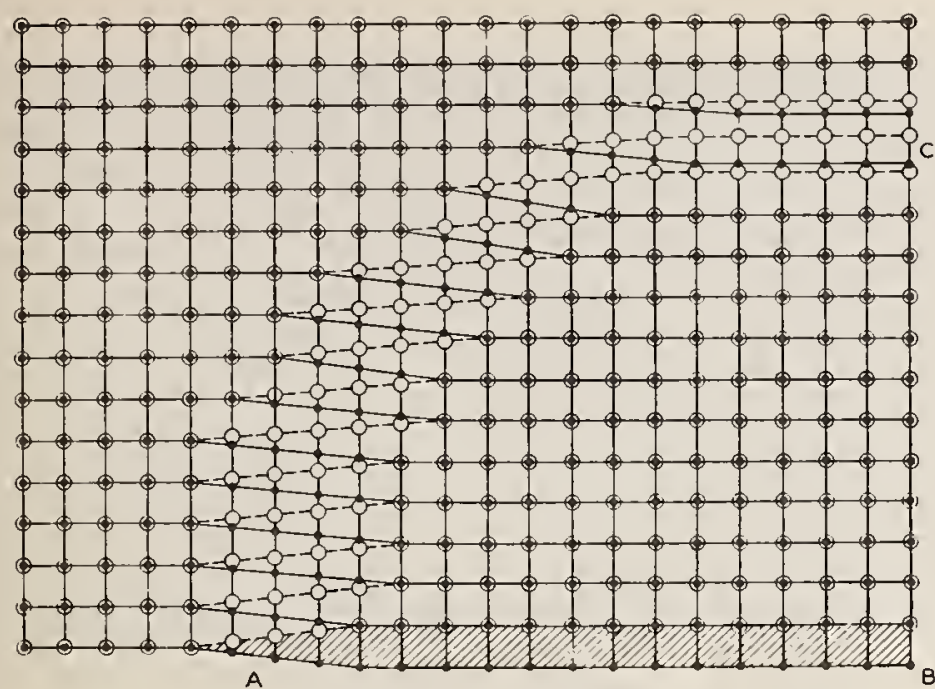

FIGURE 12. Schematic 11lustration of atomic positions along dislocation in cubic lattice.

(courtesy of W. T. Read [147].)

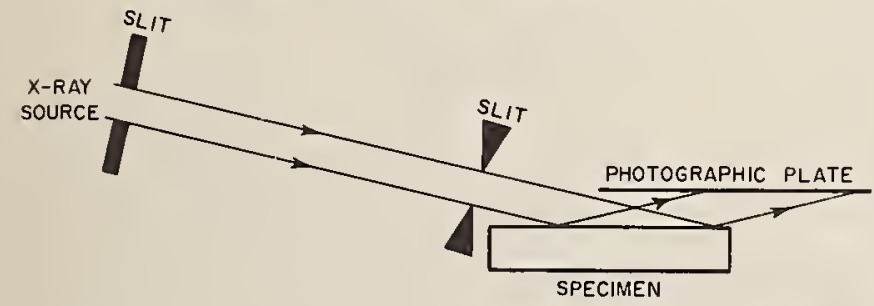

FIGURE 14. Principle of BergBarrett technique.
FIGURE 15. Profection topograph showing dislocations in silicon $(13 \mathrm{X})$.

(courtesy of A.R. Lang [75].)

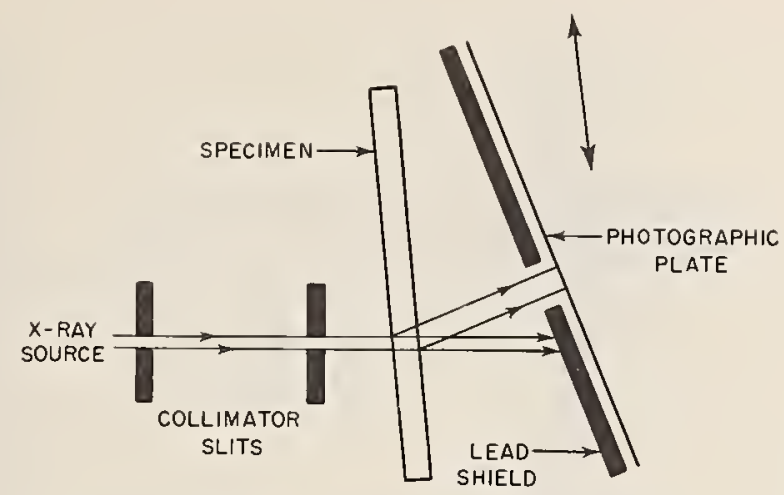

FIGURE 13; Schematic drawing to Lang's "projection topography" method. 


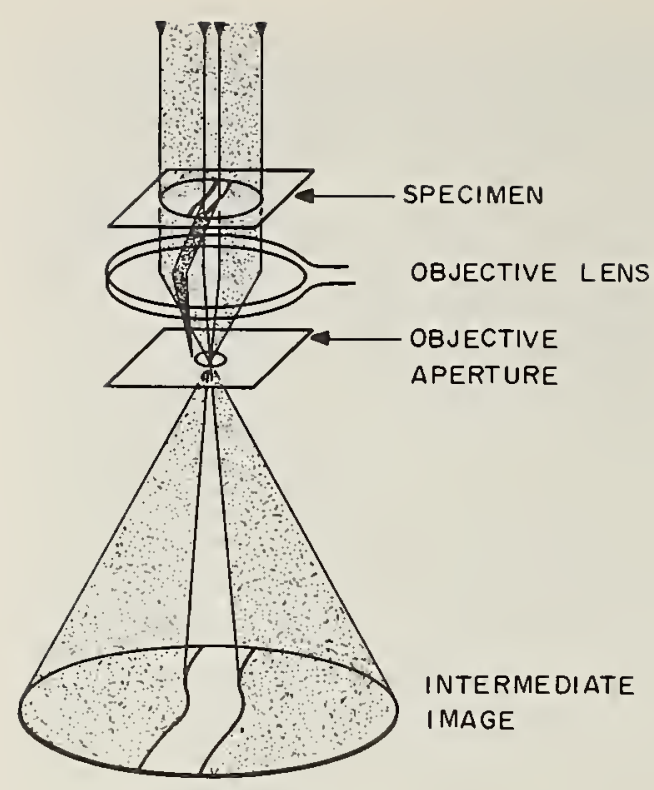

FIGURE 17. Schematic drawing of path of electrons in electron microscope which are reflected under Bragg angle by part of single crystal specimen, and which are held back by the objective aperture.

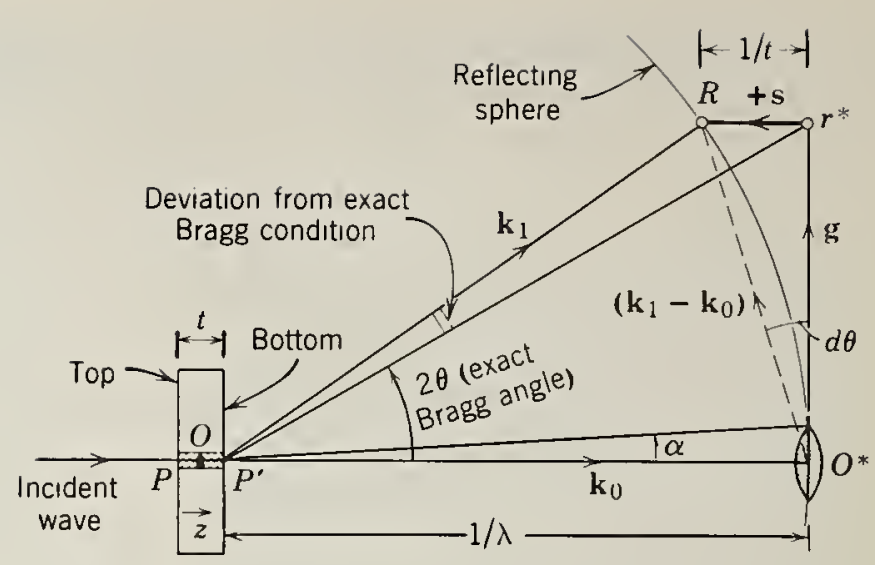

FIGURE 18. Drawing explaining symbols used in eqs (2) and (3). (courtesy of G. Thomas [79].)

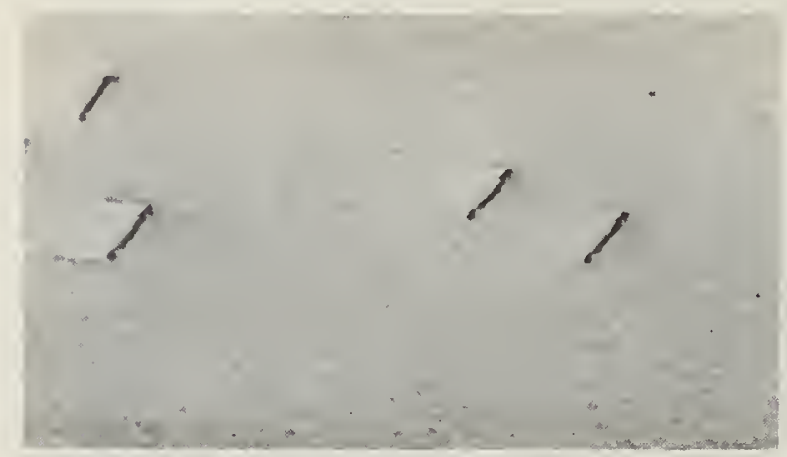

(a)

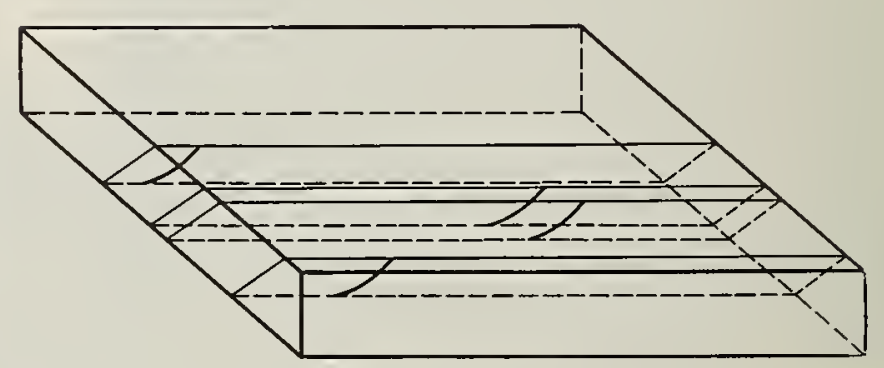

(b)

FIGURE 19.

a Glide dislocations in a thin foil of stainless steel as seen with the electron microscope $(37,500 \mathrm{x})$.

b Schematic drawing showing spatial distribution of dislocations in figure $19 a$.

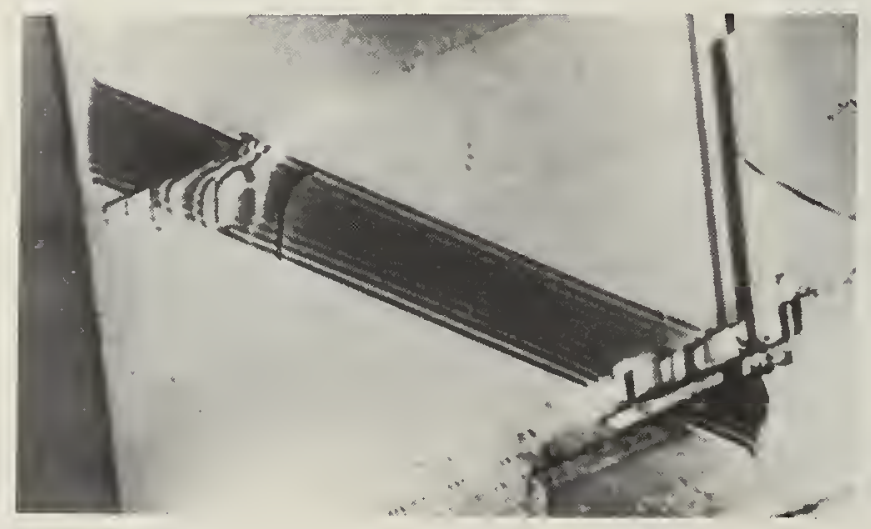

FIGURE 20. Stacking fault in thin foll of stainless steel deformed in the electron microscope $(30,000 x)$. 


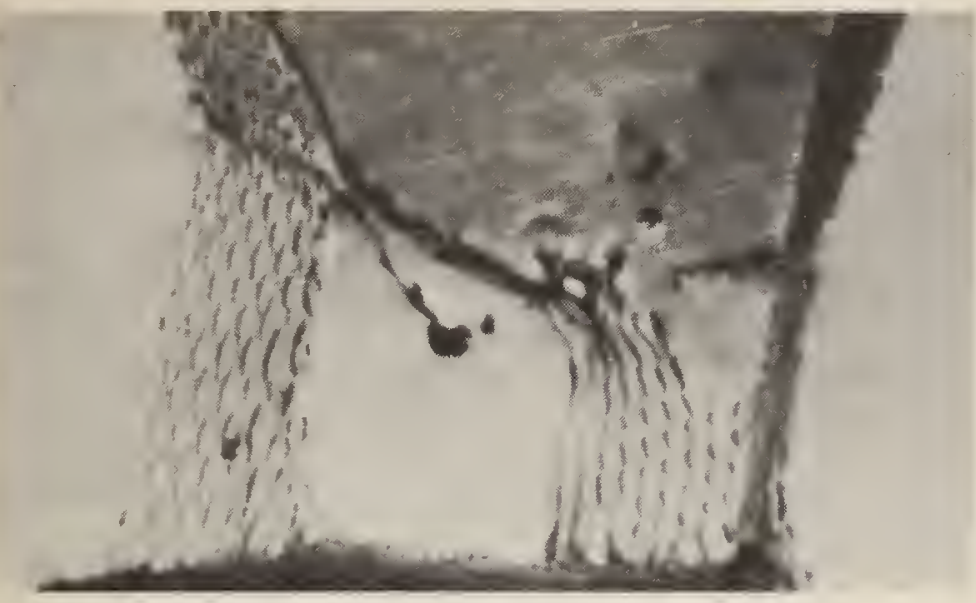

FIGURE 21. Low-angle dislocation boundaries in annealed aluminum.
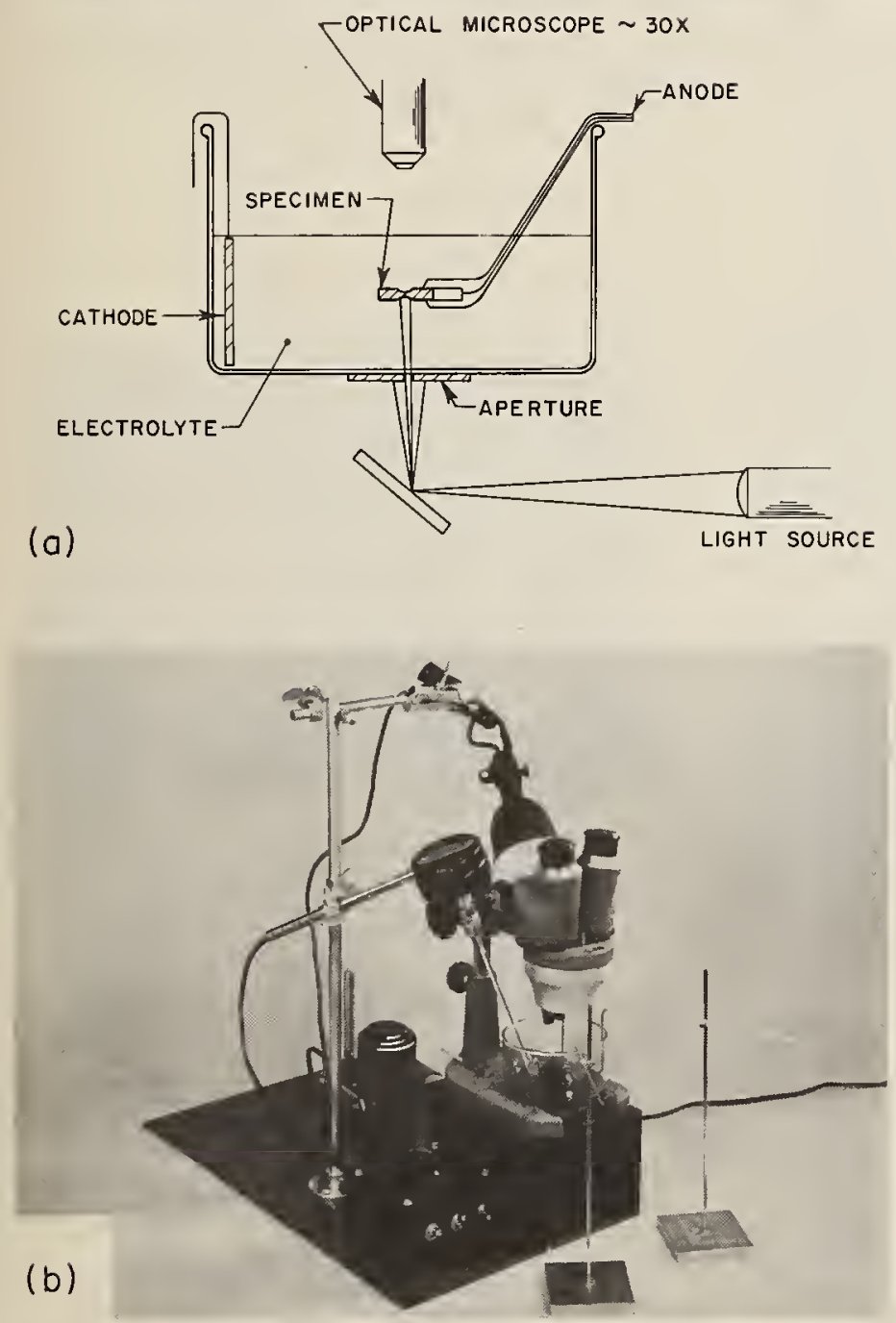

FIGURE 22.

a Schematic drawing of polishing apparatus for preparing thin specimens used in diffraction electron microscopy. (courtesy of I. Greenfield.)

b overall view of polishing apparatus .

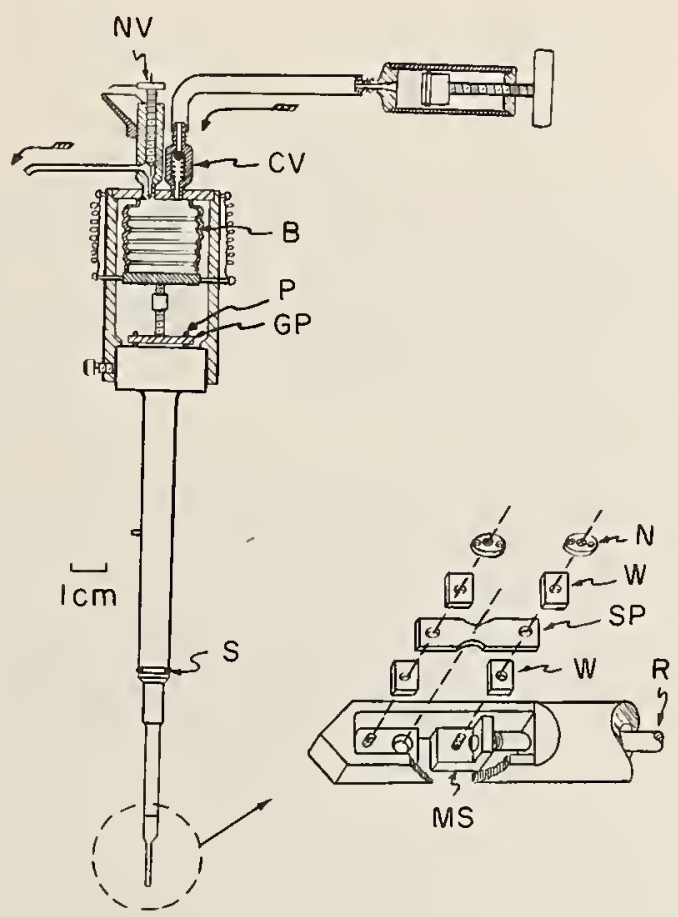

FIGURE 23. Device for straining thin foils in the electron microscope [82].

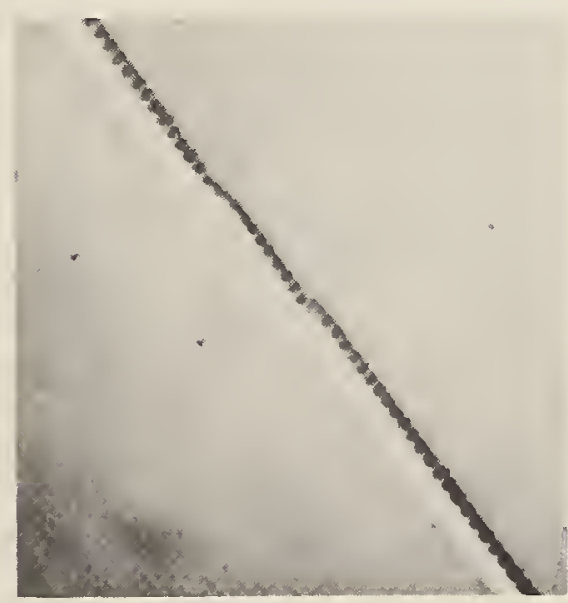

FIGURE 24. Tilt boundary in germanium outlined by etch pits. (courtesy of F. Vogel [94].) 


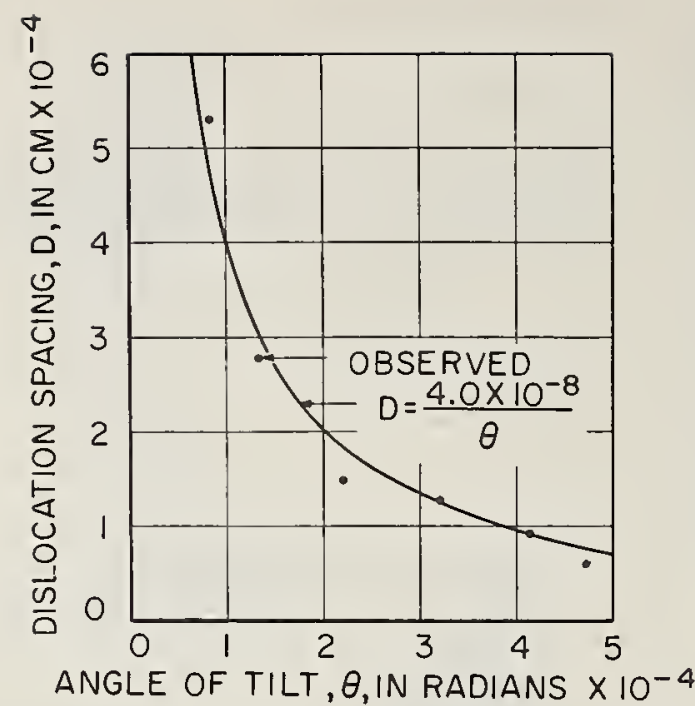

FIGURE 25. Comparison of angle of tilt obtained by etch pit technique and $\mathrm{X}$-ray method.

(courtesy of F. Voge1 [94].)

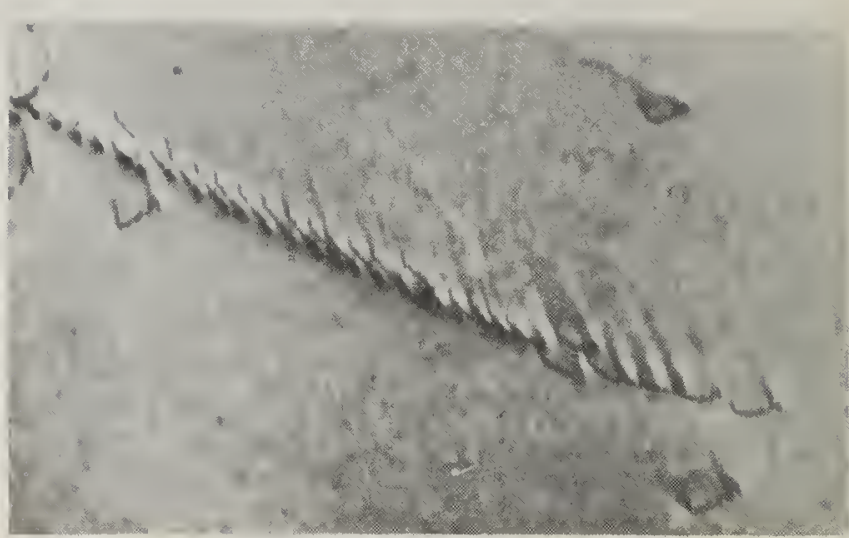

FIGURE 26. Position of dislocations in polygonization wa11; transmission electron microscopy $(52,500 \mathrm{X})$.

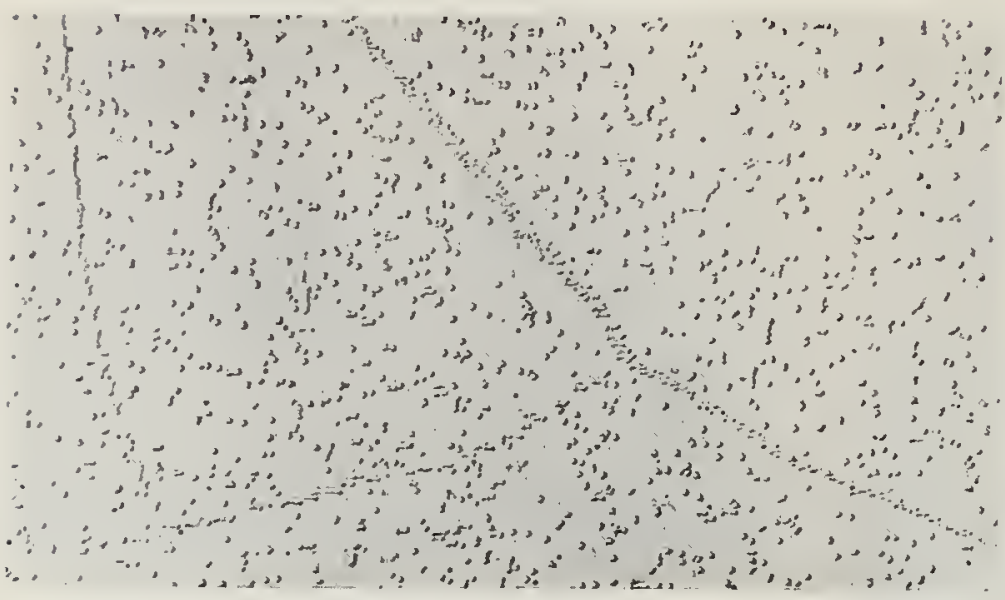

FIGURE 27. Dislocations in subboundaries marked by precipitates. A1-2\% Cu $(7,500 x[33])$.

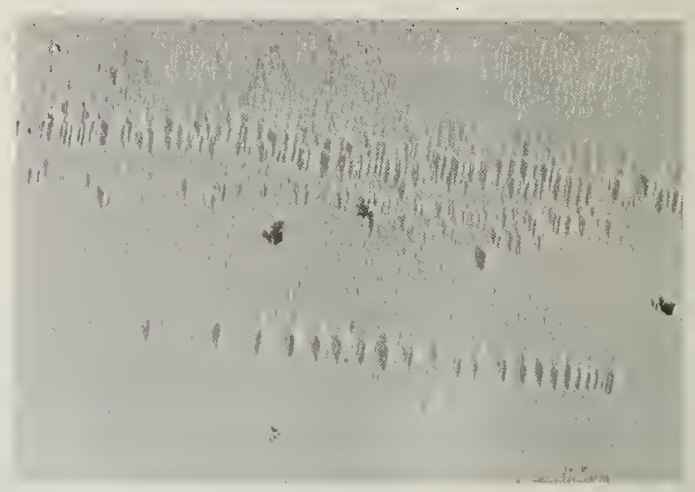

FIGURE 28. Pileup of dislocations in $\alpha$-brass, indicated by etch pits after decoration.

(Micrograph by J.D. Meakin [42].)

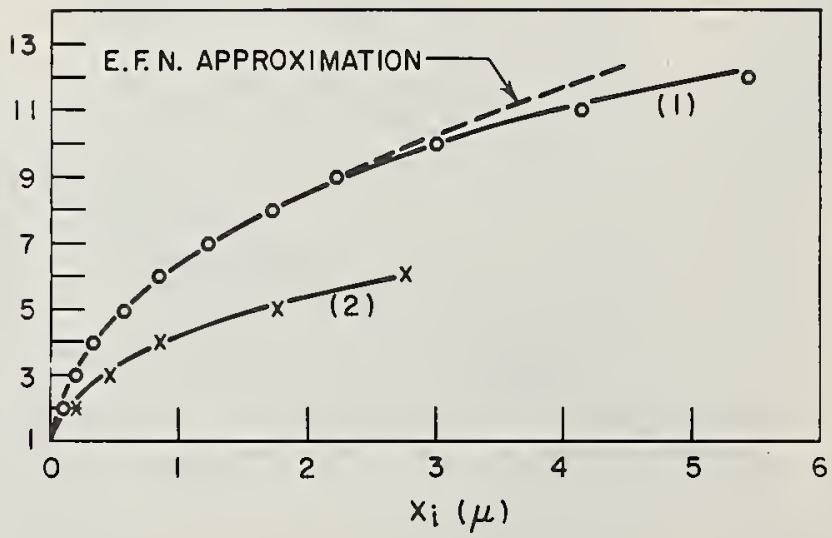

FIGURE 29. Positions of dislocations in pileups found by experiment agree with values deduced theoretically [42]. 


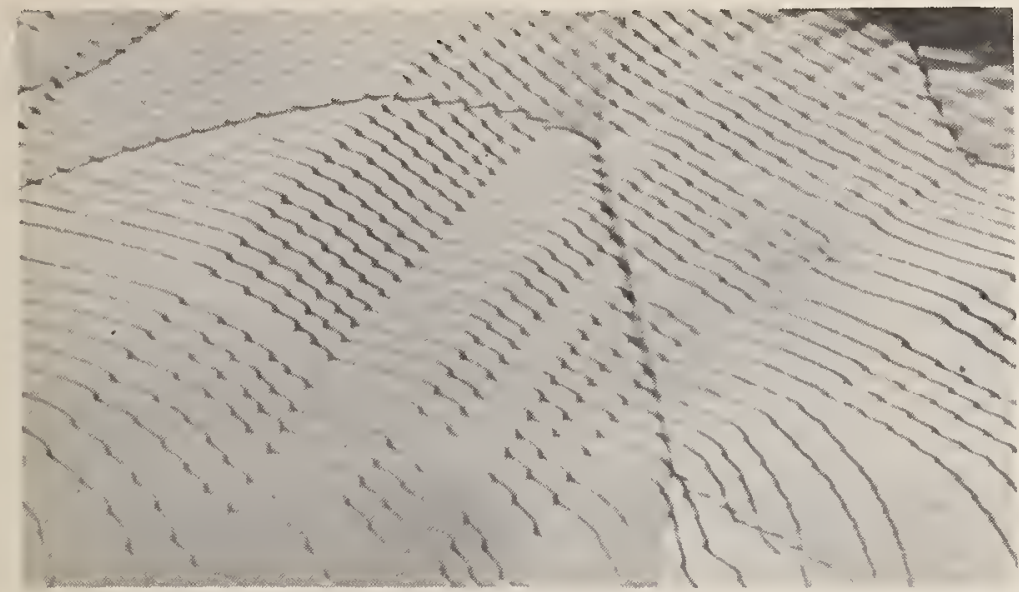

FIGURE 30. Dislocation network parallel to cleavage plane in $67 \mathrm{Bi}_{2} \mathrm{Te}_{3}-33 \mathrm{Sb}_{2} \mathrm{Te}_{3}$ $(30,000 \mathrm{X})$.

(courtesy of J. Bierly and $B . H$. Kear.)

FIGURE 31. Repeated crossslip of screw dislocation caused by obstacles in its path. Quenched aluminum $(30,000 \mathrm{X})$.

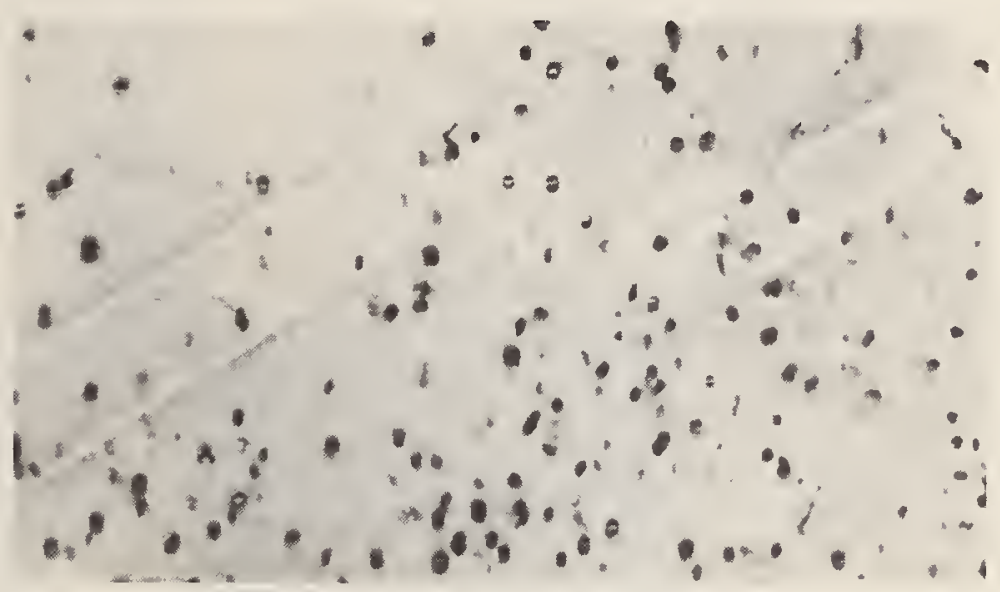

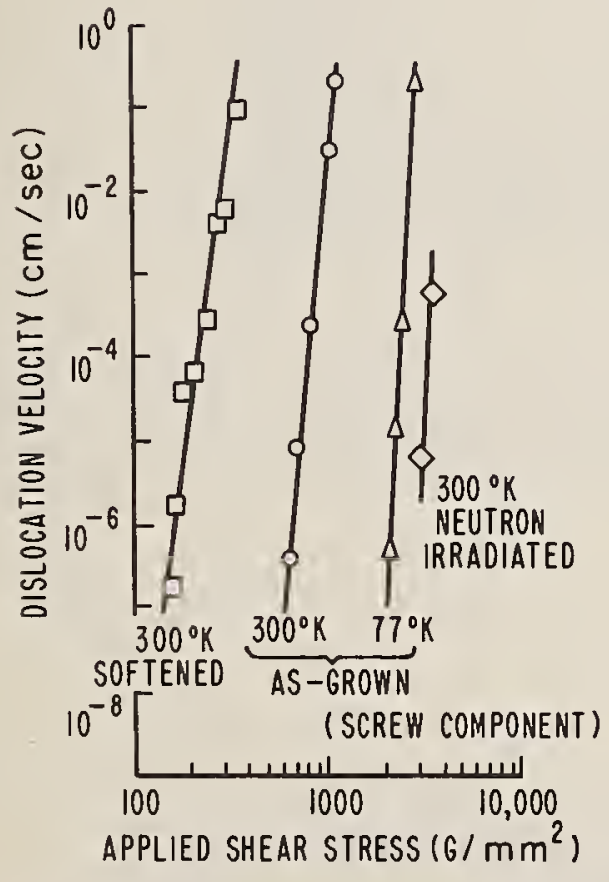

FIGURE 32. Dependence of dislocation velocity on stress in lithium fluoride.

(courtesy of $W$. G. Johnston and J. J. Gilman [105].)

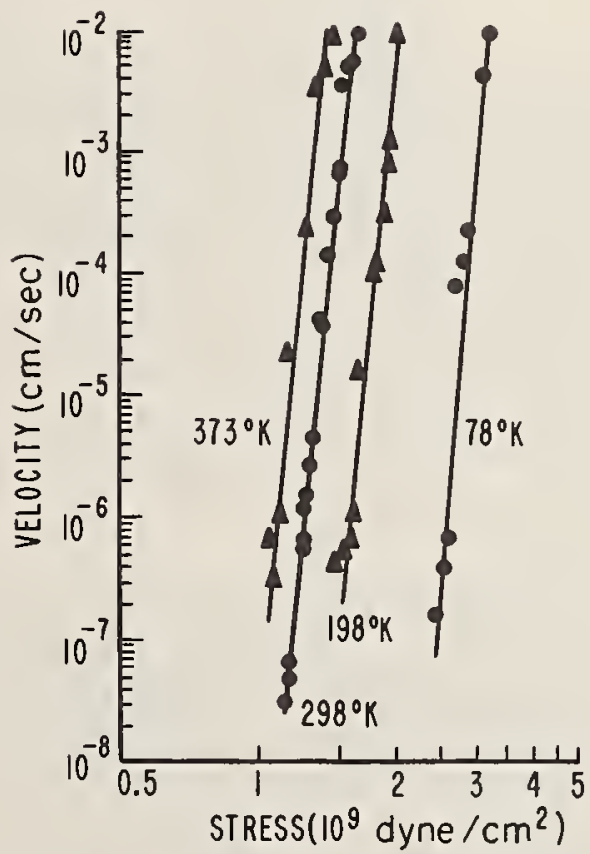

FIGURE 33. Dependence of dislocation velocity on stress in silicon-iron.

(courtesy of D. F. Stein and J. R. LOW [106].) 


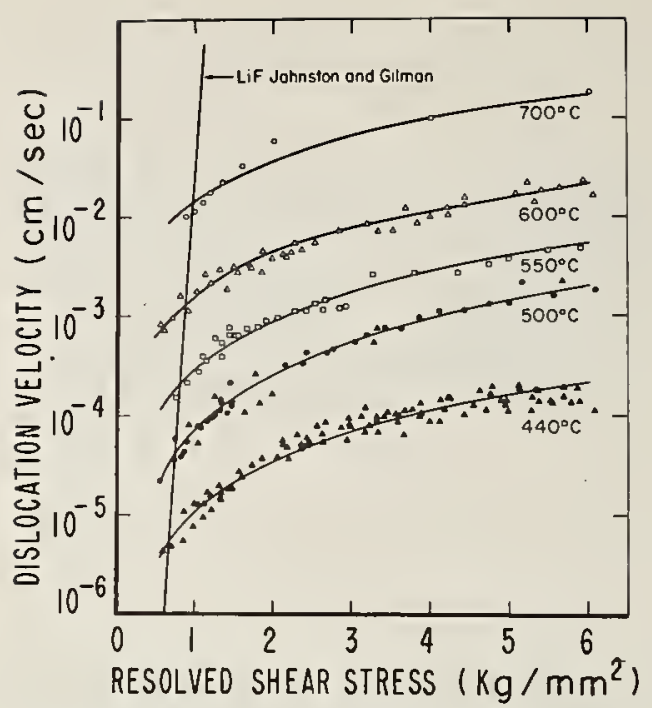

FIGURE 34. Dependence of dislocation velocity on stress in germanium.

(courtesy of A. R. Chaudhuri, J. R. Patel, and

L. G. Rubin [107].)

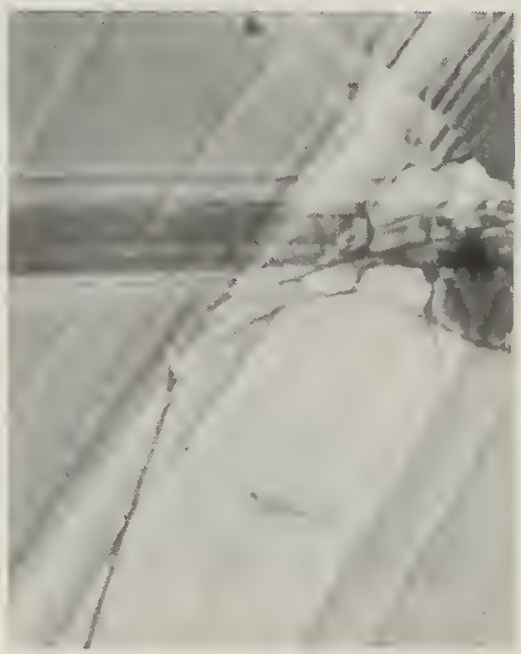

(a)

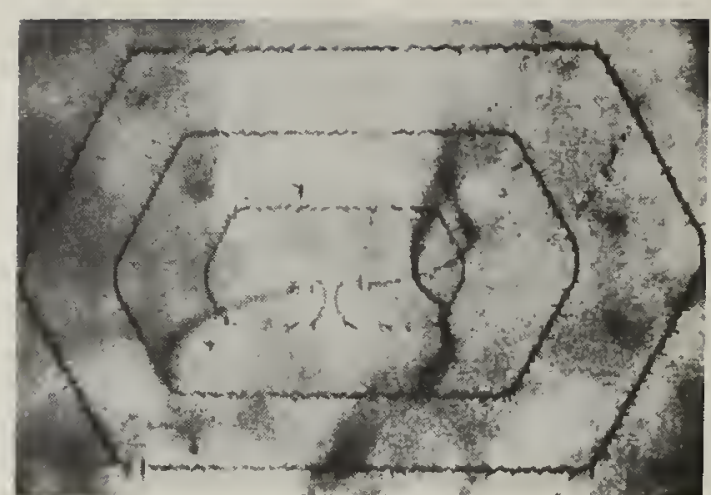

FIGURE 35. Frank-Read source in silicon. Photograph obtained by infrared technique.

(courtesy of W.C. Dash [24].)

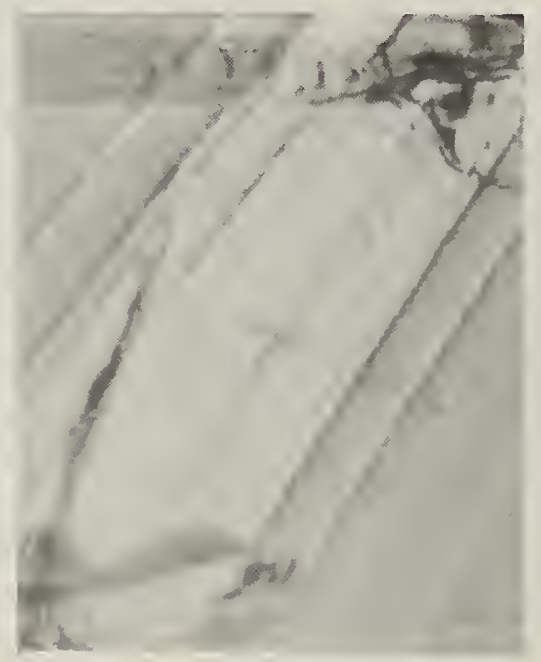

(b)

FIGURE 36. Spiral Frank-Read source operating in a thin foil of stainless steel strained in the electron microscope [118] (30,000X) ( $a$ and $b$ represent two stages in the operation of the source).

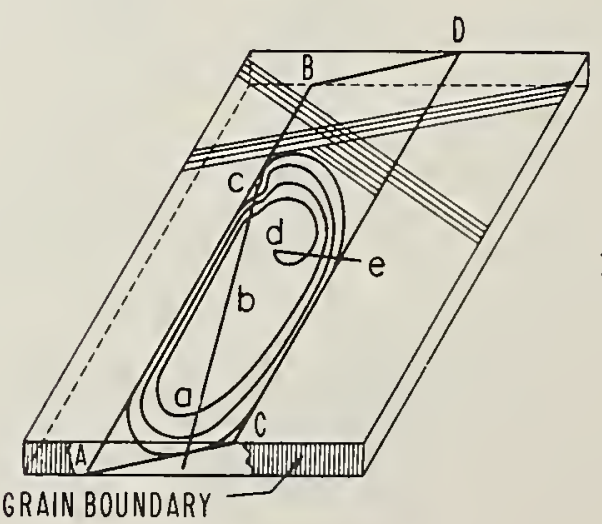

FIGURE 37. Drawing of spiral

Frank-Read source as shown in figure 36 . 

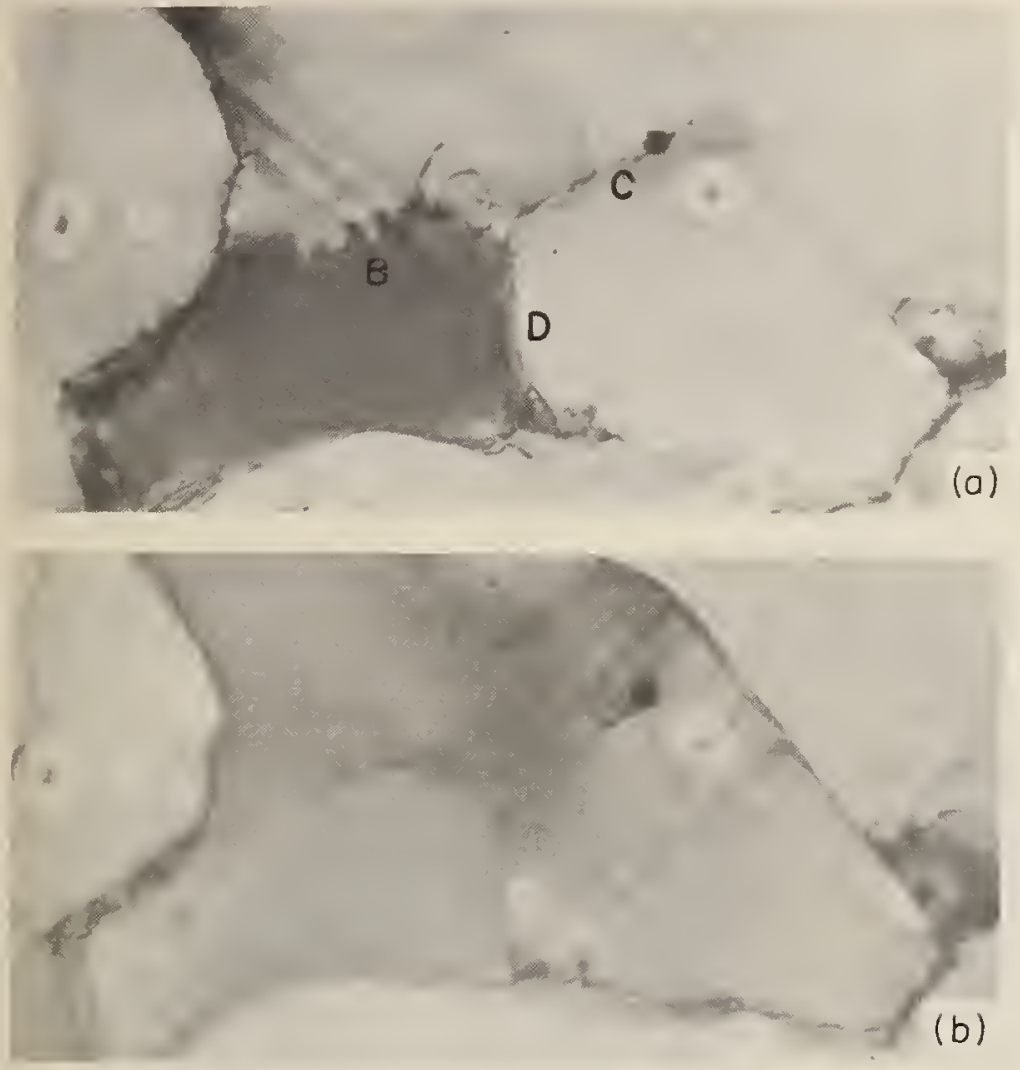

FIGURE 38. Dislocations are emitted from subboundaries in thin foil of aluminum [81] $(37,500 \mathrm{x})$ ( $\mathrm{a}$ and $\mathrm{b}$ represent successive stages in this process).

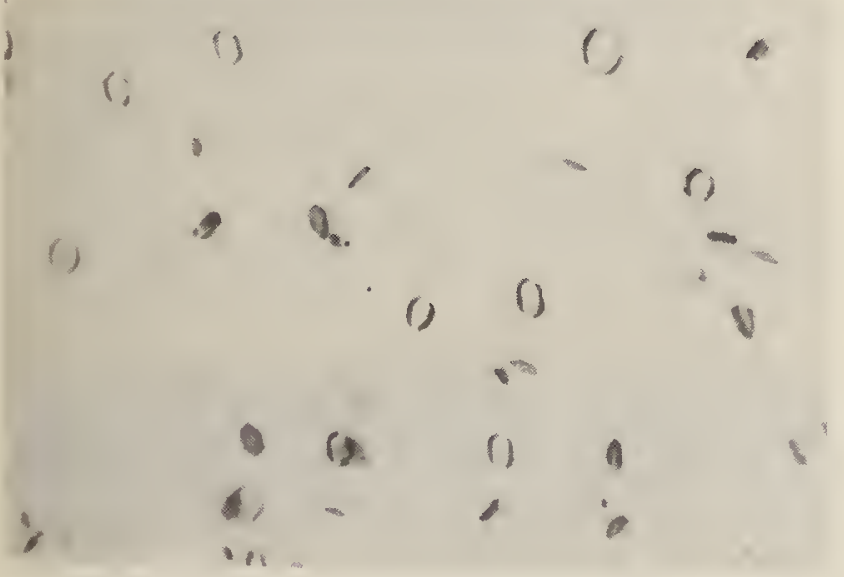

FIGURE 41. "Ouench 1oops" in aluminum [125] (45,000X).

FIGURE 42. Operation of "quench loops" as dislocation source in thin aluminum foil [115] $(33,750 x)$ ( $a$ and $b$ represent successive stages).

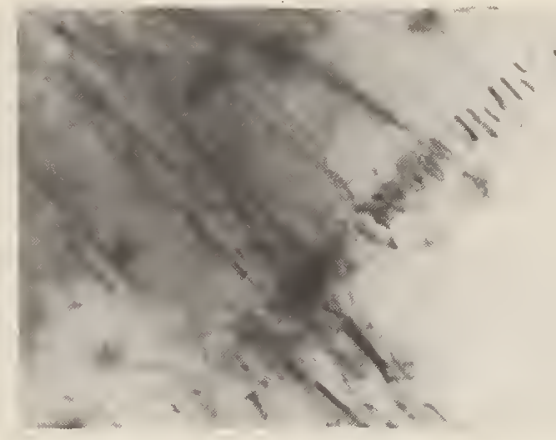

FIGURE 39. Emission of prismatic dislocation loops in bulk specimen of beryliium due to stresses at precipitate.

(courtesy of

F. Wilhelm [122].)
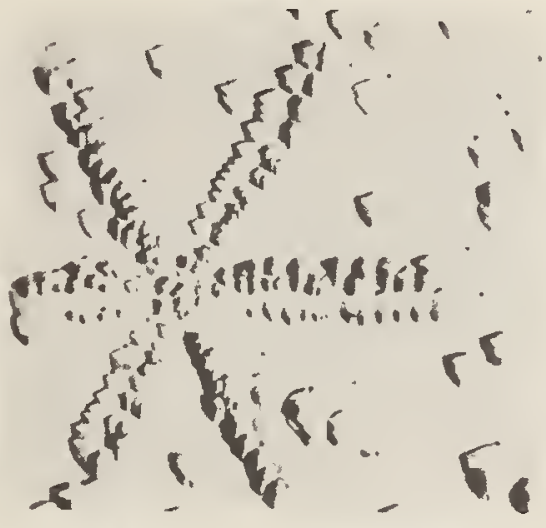

FIGURE 40. Dislocation

"rosette" on copper surface. (courtesy of $\mathrm{J} \cdot \mathrm{D}$.
Livingston $\left[60^{\circ}\right]$.)
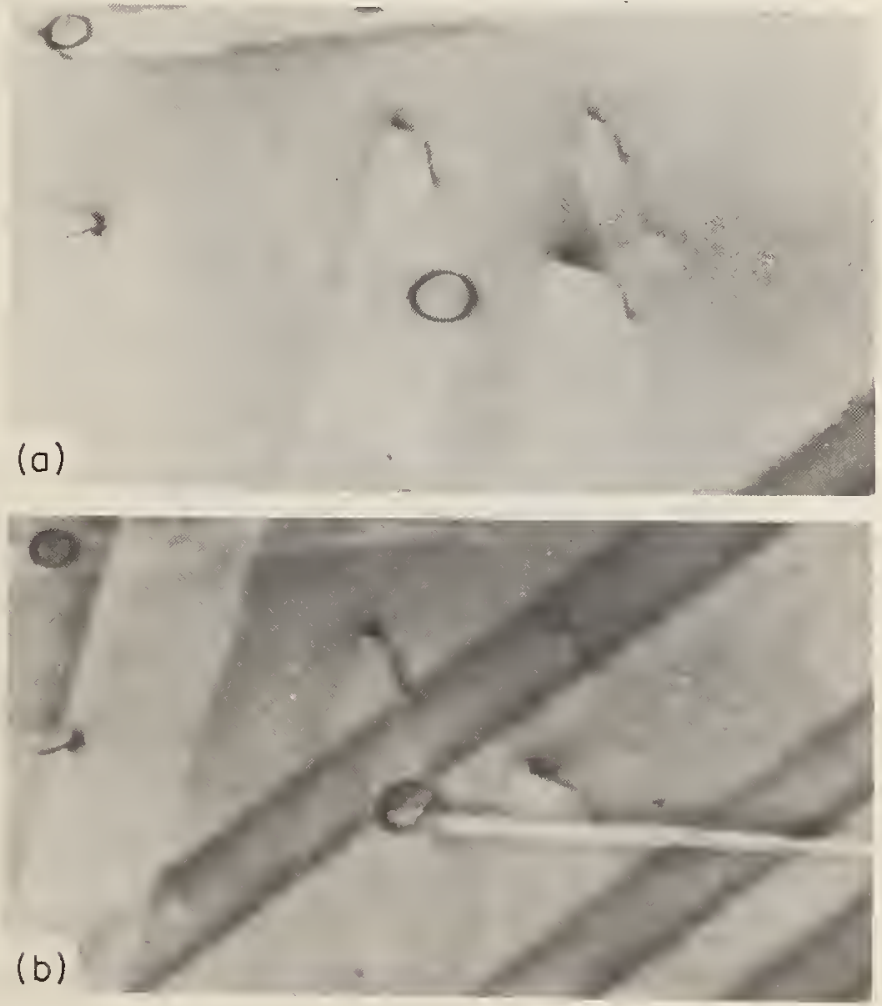


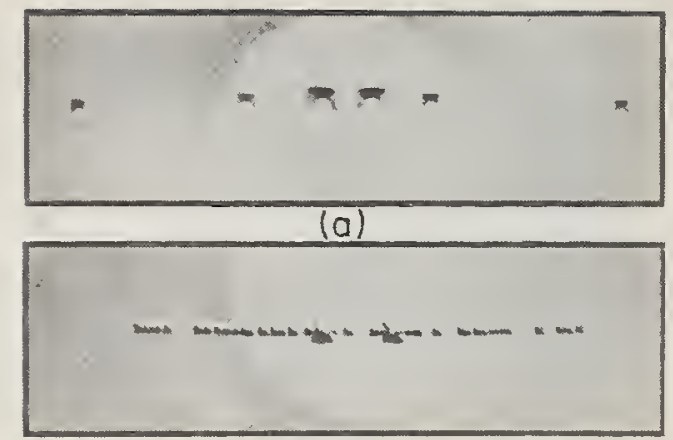

(b)

FIGURE 43. Sequence of micrographs demonstrates the multiplication of dislocations in a "slip line" ( $a$ and $b$ represent successive stages).

(courtesy of W. G. Johnston and J. J. Gilman [129].)

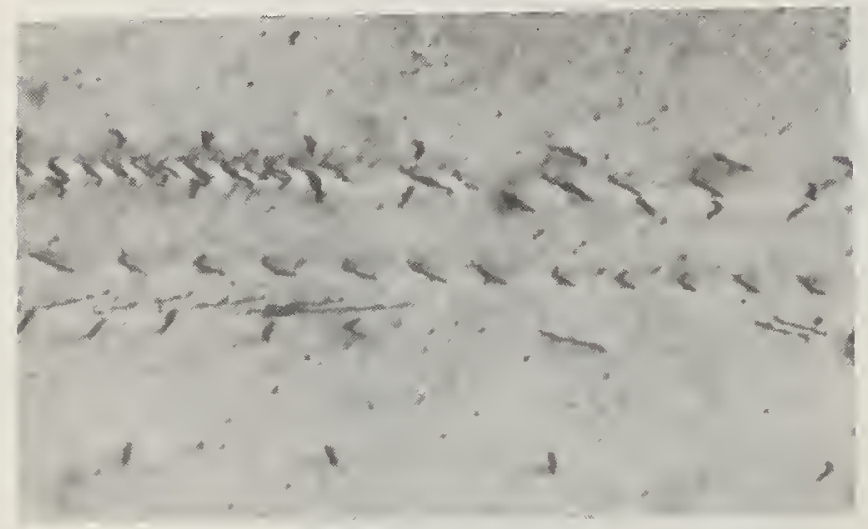

FIGURE 45. Dislocations in $\alpha$-brass are arranged in large numbers on one glide plane. Elongation $8 \%$ $(30,000 x)$.

(courtesy of J. Campbe11.)

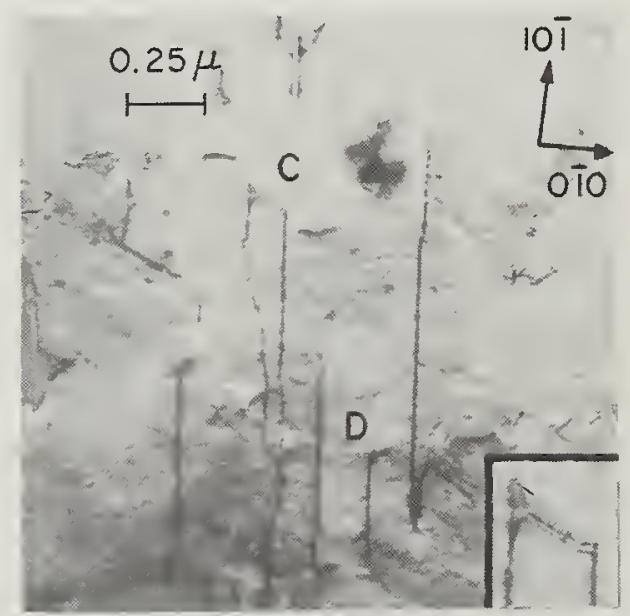

FIGURE 47. Dislocations in ordered $\mathrm{Cu}_{3} \mathrm{Au}$. Note that the super dislocations are clearly resolved when not parallel to [101]; para1lel to this direction, they have cross-slipped and are seen "head on."

(courtesy of B. H. Kear [138].)

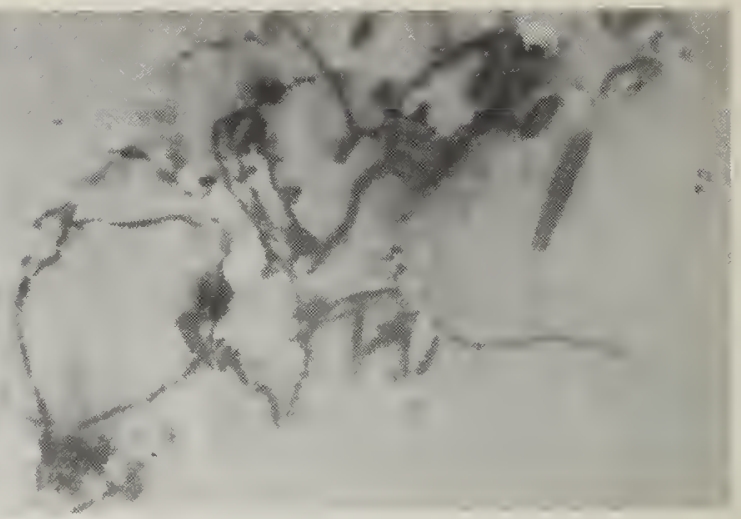

FIGURE 44. Dislocation "tangle" in aluminum single crystal deformed $10 \%$ in single slip $(37,500 \mathrm{X})$.

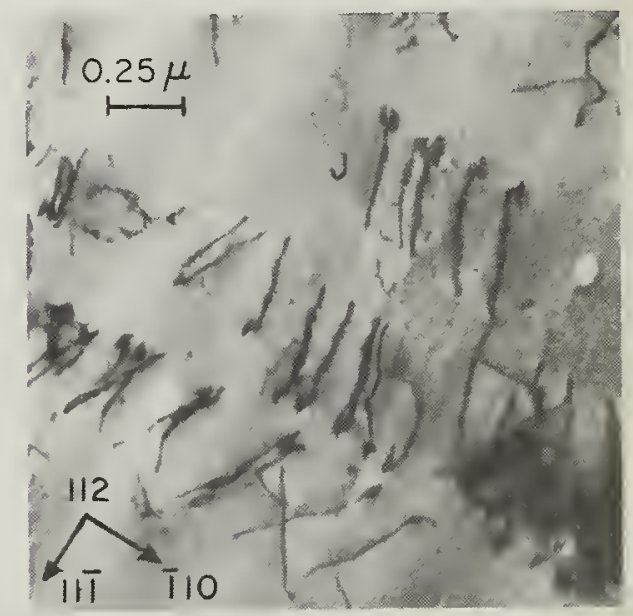

FIGURE 46. Dislocations in disordered $\mathrm{Cu}_{3} \mathrm{Au}$. Note that dislocations are arranged in groups on slip planes.

(courtesy of B. H. Kear [138].)

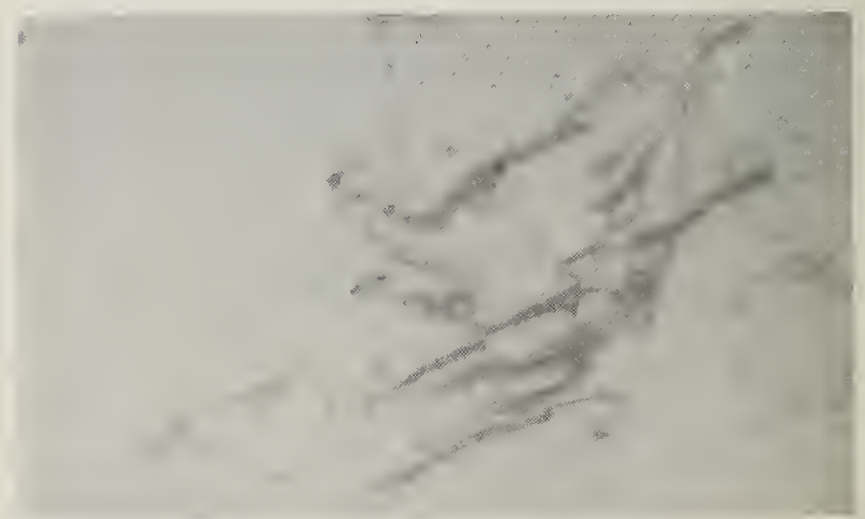

FIGURE 48. Long, narrow dislocation loops formed behind screw dislocations in aluminum single crystal. Long loops are paral$1 \mathrm{e} 1$ to $<112>$ [136]. 
T. L. Johnston

Scientific Laboratory

Ford Motor Company

Dearborn, Michigan

A description is given of the observed modes by which cracks form in crystalline ceramics as a consequence of mechanical damage and plastic flow. The stress necessary to form and propagate a crack is considered in terms of the stress concentrations which exist at the tip of a blocked glide band and at the edge of an advancing crack; particular attention is given to the influence of plastic flow at the crack tip.

\section{Introduction}

Brittleness represents the most striking feature of the mechanical behavior of crystalline ceramics. The explanation of this unfortunate characteristic is often based upon the broad generalization that ceramics are brittle because they are Incapable of deformation. Although this explanation is adequate for many materials, such as barium titanate, titanium carbide, and sapphire (at low temperatures), it is not correct for magnesium oxide. In the latter solid, dislocations are mobile, and macroscopic deformation can precede fracture under certain conditions. The reason why magnesium oxide is still quite brittle in an engineering sense is more particularly related to the inability to deform where it counts the most, namely, at the tip of a running crack.

In this paper the underlying factors which control fracture behavior of ionic solids will be described in three principal sections. In the first, an estimate is given of the theoretical strength of a solid. The second section considers how cracks are introduced and how they affect the behavior of crystals in which dislocations are essentially immobile and those in which dislocations move at relatively low stresses. The third deals with the stress required to extend a crack, and a discussion is given of the effect of plastic deformation which may accompany crack growth.

\section{Theoretical Bond Strength}

Since both the nucleation and the growth of a crack in a crystal requires that bonds be successively stretched and broken, it is important to appreciate the physical parameters which determine cohesive strength. A means by which the strength of interatomic bonds may be estimated, $[1,2]^{1}$ is provided by the change in potential energy $\mathrm{u}(\mathrm{x})$ as two adjacent planes in an ionic crystal are pulled beyond their equilibrium distance "a," (see figure la). The increase in potential energy arises from the work done against the electrostatic attraction which varies as $-\alpha z^{2} e^{2} / r$ per ion pair where $\alpha$ is the Madelung constant, $z$ the valence number, $e$ the unit of electrostatic charge, and $r$ the distance between the adjacent planes.

The stress necessary to separate such adjacent surfaces varies as the slope $d u / d r$. At the equilibrium interionic distance, the stress is zero and rises to a maximum $\sigma_{\mathrm{m}}$ at the point of inflection on the potential energy curve, (see figure $1 \mathrm{~b}$ ). It is generally assumed that the form of the $\sigma(x)$ curve is sinusoidal for values of o<x>a, i.e.,

$$
\sigma=\sigma_{\mathrm{m}} \sin \frac{2 \pi x}{\lambda},
$$

where $\lambda$ is a separation parameter in the direction of $\mathrm{x}$ which describes the range of force interaction between the surfaces. For small displacements,

$$
\sigma=E \cdot \frac{x}{a},
$$

where $E$ is Young's modulus.

$$
\text { From eq. (1), } \begin{aligned}
\frac{d \sigma}{d x} & =\frac{2 \pi}{\lambda} \sigma_{m} \cos \frac{2 \pi x}{\lambda} \\
& =\frac{2 \pi \sigma_{m}}{\lambda}
\end{aligned}
$$

\footnotetext{
${ }^{2}$ Figures in brackets indicate the literature references on page 72 .
} 
for small displacements. From eq. (2), $\frac{2 \pi}{\lambda} \sigma_{m}=\frac{E}{a}$,

$$
\sigma_{\mathrm{m}}=\frac{\mathrm{E} \lambda}{2 \pi \mathrm{a}}
$$

For an order of magnitude estimate of $\sigma_{m}, \lambda / 2$ may be taken as equal to $a$, so that $\sigma_{\mathrm{m}} \simeq \mathrm{E} / \pi$. The extent to which $\lambda / 2$ deviates from a will depend on the degree of ionic or covalent bonding. One can avoid the difficulty of assigning a value to $\lambda$ by assuming further that the work done to create unit area of surface reappears as the surface energy, $2 \gamma$, of the two surfaces produced. Thus, $\int_{0}^{\lambda / 2} \sigma_{\mathrm{m}} \sin \frac{2 \pi \mathrm{x}}{\lambda} \mathrm{dx}=2 \lambda$, $1 . e$. , $\frac{\mathrm{m}}{\pi}=2 \lambda$. From eq. (3), $\lambda=\frac{2 \pi \mathrm{m}_{\mathrm{m}}^{\mathrm{a}}}{\mathrm{E}}$ so that,

$$
\sigma_{\mathrm{m}}=(\mathrm{E} \gamma / \mathrm{a})^{1 / 2} \text {. }
$$

It is well known that the cohesive strength of a crystal is anisotropic -- most ionic crystals having well defined planes of easy cleavage. According to eq. 3 , the preferred cleavage plane should be that with minimum elastic stiffness normal to itself, maximum equilibrium separation distance, and minimum force-interaction distance. On this basis, the observed planes of easy cleavage can be correctly predicted for simple ionic solids [3]. Structural defects such as a grain boundary, an interphase boundary, or a sheet of dislocations may introduce surfaces across which the cohesive strength is lower than that of the observed cleavage plane. Defect surfaces can therefore play an important role in influencing the path taken by an advancing crack.

For the separation of $\{100\}$ planes in magnesium oxide, (4) gives a value of $\sigma_{m}$ equal to $3.7 \times 10^{11}$ dynes $/ \mathrm{cm}^{2}$, or $5 \times 10^{8} \mathrm{psi}$, if one takes values of $24.5 \mathrm{x} 10^{11} \mathrm{~m}$ dynes $/ \mathrm{cm}^{2}$ for $\mathrm{E}_{<100>}, 2.1 \times 10^{-8} \mathrm{~cm}$ for a $\{100\}$, and $1200 \mathrm{ergs} / \mathrm{cm}^{2}$ for $\gamma$. The latter value for $\gamma$ is one measured experimentally [4] which agrees fairly well with an approximate value of $1400 \mathrm{ergs} / \mathrm{cm}^{2}$ obtained by calculation on the basis of ionic theory [5]. In practice the bulk strength of magnesium oxide, like other crystalline solids, falis far short of the theoretical strength. The difference, or gap, between the experimental and theoretical values is, in fact, made up by concentrations of stress provided by pre-existing flaws and cracks or by heterogeneous plastic shear. An appreciation of the nature of the source of stress concentration and the manner in which such sources are introduced is important to an understanding of the mechanical behavior of ceramic materials.

\section{Crack Formation}

\subsection{Mechanical Damage}

The most important source of cracks or flaws in crystalline ceramics is the external surface, which is readily prone to damage by mechanical contact or abrasion. The mere act of handling a single or polycrystalline material can introduce cracks or microindentation. The final shaping of a ceramic component is frequently accomplished by a grinding process in which the surface is abraded, for example, by hard silicon carbide particles embedded in a rapidly rotating wheel. Figure $2 \mathrm{a}$ shows the damage introduced into the surface of a polished magnesium oxide crystal by the impact of a single silicon carbide particle about $1 / 16 \mathrm{in}$. in diameter dropped from a height of 2 in. The crater can be seen to be assoclated with many cracks several microns in length and an extensive zone of plastic deformation (figure $2 b$ ). Even with relatively gentle polishing procedures adopted for subsequent microscopic observation, surface damage in polycrystals may take the form of grain pul1-out by which whole grains are removed by intergranular separation. In sintered materials, additional but less severe flaws are inevitably present; residual pores and thermal grooves at grain-boundary-external surface intersections serve as moderate stress raisers.

The surface condition of a ceramic is extremely important, for the existence or absence of flaws determine in large measure the subsequent mechanical behavior of the material. In those solids in which dislocations are difficult to move, for example, aluminum oxide at low temperatures, barium titanate, titanium carbide, the size and distribution of pre-existing cracks determine in a statistical sense [6] the effective fracture strength. For this class of materials, particularly those having a high modulus, it is significant that if one takes care to prepare single crystals with flaw free surfaces, extremely high strengths can be obtained. This topic will be discussed further by J. J. Gilman at this Symposium.

\subsection{The Role of Plastic Flow}

The effects of surface condition are rather more complicated in ceramics such as 
magnesium oxide which are capable of plastic deformation. In these materials, plastic flow processes themselves are capable of nucleating cracks so that even in the absence of flaws, the fracture stress may be quite low if dislocations can move and interact at low shear stresses. For clarity, crack formation will be discussed in two general cases, I, solids having flaw-free chemically polished surfaces and II, solids having surface cracks.

\subsection{Behavior of Polished Crystals}

There are three aspects of fracture behavior which merit separate attention:

(a) The observed modes of crack nucleation.

(b) The total plastic strain which precedes fracture.

(c) The stress level at which cracks form.

\section{a. Crack Nucleation as a Consequence of Plastic Flow}

The process of plastic deformation or slip can generate a cleavage crack in a number of ways. In each case a tensile stress concentration is provided by a region of localized shear, such as a glide band, embedded in a matrix which may be plasticallystrained to a smaller degree or only elastically strained. For example, figure 3 shows the stress concentrations (revealed by stress-induced birefringence) which arise when slip bands in magnesium oxide are blocked at a grain boundary. Concentrations of stress such as these have been observed [7] to nucleate cracks (see figure 4) provided that stress relaxation does not occur first through the nucleation of glide in the neighboring grain; the propagation of glide rather than fracture is favored at simple tilt or small angle boundaries.

Varlous crack configurations have been observed some of which are shown schematically in figure 5. For example the crack may grow along the boundary as in figures 4, $5 a$, and $5 c$, or it may propagate into the neighboring grain by $\{100\}$ or $\{110\}$ cleavage (figure 5b). Furthermore, it is sometimes observed that cracks spread from the boundary along the nucleating glide band itself. The choice of configuration appears to depend upon the direction and sign of the principal tension stress with respect to the plane of the boundary and upon the degree of misorientation across the boundary (see figure 5). It will be noted that in tension, cracks may grow intergranularly from the nucleating glide band for moderate misorientations whereas in compression the intergranular path is possible only for large angle boundaries.

A crack formed at a grain boundary in magnesium oxide may also result from the interaction of two edge slip bands (one from each grain) which intersect the boundary a few microns (or less) apart (see figure 6) [8]. For this mode of crack formation to be observed the two bands must be so disposed that the concentrated tensile stresses as sociated with each band superimpose. In figure 6 , if the intersection $B$ of glide band $A^{\prime} B '$ had been on the right-hand side of $B$, a crack would not form by this mechanism.

Surface deposits or reaction products [9] can provide interfaces at which cracks may form. For sodium chloride and magnesium oxide, it has been demonstrated that when a stain or deposit arises from inadequate washing and drying procedures, cracks develop after plastic yielding at the interface between the crystal and the deposit and spread into the crystal (figure 7) [10]. Only those deposits which lie on surfaces through which edge dislocations emerge are effective in forming cracks.

Another example of crack nucleation at a boundary is one in which the latter is a kink (across which there is lattice rotation) generated by plastic flow itself. When cubically oriented rock-salt type single crystals are plastically compressed [11,12] or bent [13], kinks may develop as a result of loading (grip) or geometrical constraints. In bending, the constraints arise from a gradient in lateral contraction accompanying pronounced curvature. Figure 8 is the cleavage fracture surface of a sodium chloride crystal which had been bent at liquid nitrogen temperature [13]. A kink boundary $C D$ extending across one of the corners on the tension side of the specimen is clearly evident. The kink consists of an array of edge dislocations having $<110>$ Burgers vector and $\{112\}$ slip planes which result from the reaction or combination of $\langle 110\rangle$ edge dislocations gliding on non-orthogonal $\{110\}$ planes. (See Kear et a1. [14] for a discussion of the relevant dislocation reactions.) Under certain combinations of strain rate and temperature [13] (100) cleavage may originate at some point along such a kink or it may spread along the beam parallel to the plane of the kink. 
The intersection of orthogonal $\{110\}$ glide bands provides a source of crack formation which has been observed by a number of investigators. Two configurations are possible. In one, the crack which forms initially lies along a $\{110\}$ plane at the edge of one of the intersecting bands as shown schematically in figure $9 a[15,16]$; figure $9 \mathrm{~b}$ illustrates the second configuration in which the crack lies on a $\{100\}$ plane and spreads across the boundary of the slip band intersection $[17,18]$.

Adams and Murray [19] have shown that at temperatures in excess of $0.5 \mathrm{~T}_{\mathrm{m}}$, magnesium oxide and sodium chloride bicrystals can undergo grain boundary shear $\mathrm{m}$, remarkably high strain rates. The actual value depends upon the stress, temperature, and nature of the boundary. Simple small angle boundaries have the greater resistance to shear. In polycrystals, a result of this phenomenon is the creation of wedge-shaped voids along triple lines where a number of grains are in juxtaposition. This mode of crack formation is commonly observed in metals loaded at high temperatures at very low strain rates, but in ionic polycrystals this mode can be operative also at moderately high strain rates. Figure 10 shows the intergranular cracking produced by boundary sliding in the tension surface of a polycrystalline rod of lithium fluoride strained at $10^{-1 / \mathrm{sec}}$ at $400^{\circ} \mathrm{C}[20]$.

\section{b. Degree of Deformation Preceding Complete Fracture}

Single crystals. Cracks which form in MgO at the intersection of two orthogonal

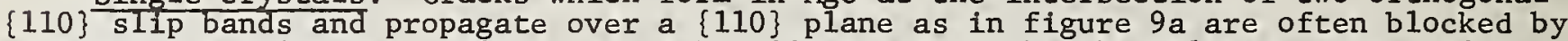
a neighboring glide band [15]. A crack will spread in the channel between two slip bands to form a narrow $\{110\}$ slit lying in a $\langle 100>$ direction. (Subsequent crack growth at a higher stress tends to occur on the customary $\{100\}$ plane.) Figure 11 shows the relation of a number of such slits to the distribution of slip bands. The ability of a glide band to arrest the growth of a crack was first observed in sodium chloride by Melankholin and Regel [21] and stems from the fact that the crack has to cut through the screw components of the very large number of dislocations $\left(10^{9} / \mathrm{cm}^{2}\right)$ making up the band (see section 3.3). A consequence of this form of crack stabilization is that the total deformation which precedes complete fracture depends critically on the distribution of slip at the onset of yielding [22]. Sometimes, for example, deformation in magnesium oxide proceeds by the nucleation and growth of a single glide band which spreads along the gauge length, in which case a glide band intersection is avoided: Such a crystal may exhibit as much as 7 percent plastic strain in tension before fracture. However, if just two glide bands happen to intersect at the onset of yielding before many other glide bands are formed, complete fracture can occur with very small macroscopic strain. Figure 12 is a macrophotograph of a polished monocrystal of magnesium oxide which failed in this way in tension after only 0.2 percent strain. On the other hand, if slip is finely spaced on two orthogonal systems respectively, not only can cracks be stabilized (allowing continued deformation up to $\sim 5$ percent strain in tension), the nucleation of cracks by this mechanism may even be suppressed altogether. When the suppression of slit formation occurs in bending, longitudinal cracks may form at corners due to kinking as shown in figure 13.

Just as the total amount of deformation preceding fracture is controlled, in part, by the distribution of slip, so does the number and spacing of slip bands depend upon the distribution of active dislocation sources. There is evidence [23] that in magnesium oxide, precipitate particles serve to nucleate dislocations internally by acting as shear stress raisers. (Gilman [24] has pointed out a similar possibility in lithium fluoride.) In the as-received condition, one cannot predict what the initial slip distribution will be. However, one can exercise a certain degree of control by altering the number of dislocation sources. For example, Stokes [23] has shown that by annealing polished magnesium oxide at $2000^{\circ} \mathrm{C}$ in nitrogen for $1 / 2 \mathrm{hr}$. one can, in effect, prevent slip from starting from "natural" sources. This is believed to be due to the dissolution of the precipitates. Crystals heat treated in this way are quite strong, having yield and fracture stresses in excess of 100,000 psi. One can choose the opposite alternative of insuring a fine distribution of slip by deliberately introducing many dislocation sources at the surface by sprinkling the specimen with 200 mesh silicon carbide [22] powder. Furthermore, an increase in temperature appears to accomplish an increase in the number of slip bands. For example, lithium fluoride fractures at liquid nitrogen temperature by the formation of stable slits in the manner of polished magnesium oxide at room temperature, but lithium fluoride at room temperature behaves like magnesium oxide in which slip is very finely spaced [20].

Polished polycrystals. Since the mechanical behavior of polycrystals will be discussed by Kingery and Coble at this symposium the only point which needs stressing in this paper is that the amount of deformation which precedes complete fracture of a rock-salt type material of given purity depends upon strain rate and temperature. Experiments with silver chloride and sodium chloride polycrystals have shown [20] that 
if the loading conditions are such that the corresponding single crystal form is notch insensitive, the polycrystals are quite ductile and localised necking precedes fracture (provided the temperature is not so high that grain boundary shear takes place). If, on the other hand, the strain rate and temperature are such that cleavage cracks can propagate readily in the single crystal, the presence of grain boundaries decreases the ductility and little or no localised necking is possible at the point of fracture. The total elongation, which reflects the amount of strain preceding crack nucleation, decreases with a decrease in temperature.

\section{c. Fracture Stress}

In section $2.3 \mathrm{a}$ a description is given of the disposition of the crack with respect to the local distribution of plastic flow and the structural features resisting dislocation motion. It should be emphasized that the corresponding underlying mechanisms in terms of dislocation interactions are by no means clear.

The generation of a crack at the tip of a blocked glide band (first suggested by Zener [24]) as illustrated in figure 5 is probably the simplest one to consider in terms of dislocations. The most straightforward criterion for crack formation is that the stress concentration at the tip of the band must locally exceed the cohesive strength of the material. Since it can be shown [26] that the stress concentration factor is equal to $n$, the number of dislocations piled up against the barrier, it follows that

$$
\sigma+\mathrm{n}\left(\sigma-\sigma_{0}\right) \geq \sigma_{\mathrm{m}} \text {, }
$$

where $\sigma$ is the applied tension stress and $\sigma$ is the tension stress whose shear component is sufficient to move dislocatbns. The value of $\sigma_{\mathrm{f}}$ will depend upon whichever initial crack path is manifested (intergranular, glide Band or transgranular cleavage). Furthermore,

$$
\mathrm{n}=\frac{(\tau-\tau \mathrm{T})}{\mu^{\mathrm{b}}} \cdot \mathrm{L} \quad[27]
$$

where $T$ is the applied shear stress, $T$ is the shear stress to expand dislocation loops, $\mu$ is the shear modulus, $b$ the Burger's vector, and $L$ is the distance between the initial dislocation source and the barrier.

The essential feature of " $n$ " is that it is structure-sensitive, and therefore depends upon the thermal-mechanical history of the solid. In other words, $n$ is influenced by purity, heat treatment, distribution of dislocation sources, precipitates, and grain size. For example, an increase in purity will decrease $T$; a decrease in grain size and a fine slip distribution will decrease the value of $Q^{\prime}$ and therefore increase the stress necessary to cause fracture. When cracks form at grain boundaries in magnesium oxide bicrystals the fracture stress is little more than the stress required to nucleate and multiply dislocations to form a densely populated glide band. Stokes and $\mathrm{Li}$ [28] have shown that a magnesium oxide bicrystal which has been heat treated at $2000{ }^{\circ} \mathrm{C}$ to eliminate "natural" dislocation sources can sustain stresses in excess of $110,000 \mathrm{psi}$ in tension. An identical bicrystal, similarly heat treated, in which fresh dislocations had been introduced within the gauge length suffered intergranular fracture at tensile stresses in the range 8,000 10,000 psi which is precisely the stress range necessary to generate slip bands from active dislocation loops. The fracture stress is therefore clearly dependent on the stress to generate a glide band.

Why cracks are nucleated at glide band intersections still awaits an unequivocal interpretation. It has been suggested [15] for example, that $\{110\}$ cracks form as a result of the pileup of dislocations moving at the edge of one of the $\{110\}$ glide bands against the orthogonal band. However, one cannot explain why many silp band intersections do not lead to the formation of cracks and why cracks form only after glide bands pass through one another. Argon and Orowan [29] have recently shown that the material in the parallelogram of the slip band intersection (see figure 9 ) undergoes lattice rotation; its boundaries therefore are kinks which can play a role similar to those described in section $2.3 a$.

The minimum stress required for complete fracture of polished monocrystals is that required for slip band generation; slightly higher stresses may be necessary according to the total amount of plastic strain, which depends on the distribution of slip and the rate of strain hardening as shown diagramatically in figure 14. Our limited understanding of the dislocation reactions involved precludes the calculation of fracture stress on the basis of a specific model. 


\subsection{Crystals Containing Microcracks}

As one might expect, the presence of cracks limits the extent of deformation prior to fracture by providing regions of high tensile stress concentration. If large cracks are present, fracture can occur at macroscopic stresses lower than that necessary to nucleate glide bands (taking into account strain rate effects to be discussed later) in which case they behave in a totally brittle fashion. When small cracks are present, and if stresses are applied slowly enough, slip can start from active dislocation loops already present in both the surface and at the tips of the pre-existing microcracks. Clarke and Sambel1 [16] have studied in some detail the role of microcracks too small to initiate premature fracture. They have shown that in magnesium oxide loaded at low strain rates, cracks can grow slowly upon gradually increasing the load after they have nucleated giide bands. It is not certain yet why they can grow in this manner; the reason may be related to the drop in the effective value of $\sigma_{m}$, the cohesive strength, across the nucleated glide bands at the tip. The crack grows therefore at a rate controlled by the progressive nucleation of slip until a size is reached at which the crack can propagate elastically.

Miles and Clarke [30] have recently emphasized the importance of microcracks in relation to the thermal shock resistance of magnesium oxide. Quenching crystals containing microcracks from $1000^{\circ} \mathrm{C}$ into water at room temperature leads to shattering which can be traced back to a single microcrack nucleus. Polished crystals do not fracture, instead thermal stresses induce finely spaced slip. In contrast, biorystals fracture upon quenching whether they are polished or not due to the greater ease with which grain boundary cracks form compared to those generated at slip band intersections.

The well-known Joffé effect [31] observed originally in sodium chloride monocrystals when they were immersed in a solvent, is due unequivocally to the elimination of surface microcracks $[10]$. In the case of sodium chloride at room temperature, such elimination always leads to an enhancement of ductility. In magnesium oxide on the other hand, the removal of microcracks does not necessarily lead to an increase in ductility at room temperature at low strain rates for the ductility depends also on the distribution of slip. Nevertheless, the source of fracture is always changed from a point at the surface associated with a defect to a point on an internal slit or kink boundary.

Even when dislocation mobility is sensibly zero as in silica or aluminum oxide at room temperature, cracks too small to propagate spontaneously can grow progressively at a constant stress through the action of an active environment such as water vapor. (Charles has discussed this topic in detail [32].) The effect is associated with chemisorption of active molecules in the region of the crack tip which results in a drop in the binding energy of the unbroken lonic bonds which constitute the crack edge. This change in binding energy leads to a different value of $\sigma_{\mathrm{m}}$ obtained from eqs. (3) and $(4)$.

\section{Crack Propagation}

\subsection{Stress Concentration Factor}

Inglis [33] pointed out that flaws or cracks which exist either internally or at the surface of a solid serve to concentrate or magnify the applied stress. For example, if a plate containing an elliptical crack of length $2 c$ is subjected to a tensile stress $\sigma$, perpendicular to the major axis of the crack, the local stress at the ends of the major axis is

$$
\sigma_{c}=2 \sigma\left(\frac{c}{p}\right)^{1 / 2} \text {, }
$$

where $\rho$ is the radius of curvature at the ends of the major axis. Orowan [2] has emphasized that there is no physical sense in considering radii of curvature less than the interionic distance. The sharpest possible cleavage crack therefore will have a value of $p$ equal to a the interionic distance. The condition for elastic crack extension is that the stress concentration $\sigma_{c}$ at the ends of the crack having $\rho=a$ must exceed the theoretical bond strength $\stackrel{c}{\mathrm{~g}}_{\mathrm{m}}, \mathrm{i} . \mathrm{e}$.,

$$
\begin{gathered}
2 \sigma\left(\frac{c}{a}\right)^{1 / 2} \geq\left(\frac{E_{\gamma}}{a}\right)^{1 / 2} \\
\sigma_{f} \geq\left(E_{\gamma} / 4 c\right)^{1 / 2} .
\end{gathered}
$$

The distribution of normal stress and the actual atom positions at the tip of a crack of length $2 \mathrm{c}$ equal to $1000 \mathrm{a}$ is shown in figure 15 based upon the calculations 
of Elliot [34] for a simple cubic crystal, whose modulus $\mathrm{E}=10^{12} \mathrm{dynes} / \mathrm{cm}^{2}$, under an applied stress of $0.01 \mathrm{E}$. The maximum stress can be seen to be of the order of $\sigma_{\mathrm{m}}$. E11iot pointed out that the variation of stress. With atomic displacement at the me of the crack, correspond to an effective force-distance law very similar in form to that shown in figure $1 \mathrm{~b}$. It is important to remember therefore that the ions such as $A B$ which constitute the unbroken bonds defining the edge of the crack undergo the same force-displacement curve as they are pulled apart. The values of $\mathrm{E}, \lambda, \mathrm{a}$, and $\gamma$ which determine $\sigma_{m}$ apply to the unbroken bonds such as $A-B$ at the crack tip.

Griffith [35] showed that it was possible to obtain eq. (8) without consideration of the geometry of the crack tip. The premise was made that the rate of elastic energy release during crack propagation must be equal to or greater than the rate of increase of surface energy of the crack surface area. The relief of strain energy associated with a crack of length $2 c$ in a thin plate subjected to a tensile stress $\sigma$ nor$\mathrm{mal}$ to the plane of the crack is given by $-\pi c^{2} \sigma^{2} / \mathrm{E}$ per unit thickness. The increase in surface energy equals $4 \mathrm{rc}$. The equilibrium crack length may be found by differentiating these expressions with respect to crack length and equating the results, i.e.,

$$
\begin{aligned}
2 \pi \sigma^{2} \mathrm{c} / \mathrm{E} & =4 \gamma \\
\mathrm{c} & =\frac{2 \mathrm{E}}{\pi \sigma^{2}},
\end{aligned}
$$

or for a given crack length, c, the critical stress for propagation $\sigma_{f}=(2 \gamma E / \pi c)^{1 / 2}$. In this case, the value of $\gamma$ refers to an equilibrium value with respect to the environment and $\mathrm{E}$ corresponds to the bulk value.

For the fracture of an elastic solid in vacuo, the respective "stress-concentration" and "thermodynamic" approaches are equivalent except for a minor numerical difference. However, if an active environment is present which has access to the crack tip or if plastic relaxation occurs there, the most straightforward way to consider such effects is use the stress-concentration method which takes into account events at the crack tip.

It should be noted that eq. (8) indicates that an atomically sharp crack will grow at those stresses normally observed (i.e., $\mathrm{E} \times 10^{-2}$ to $\mathrm{E} \times 10^{-3}$ ) if internal or surface cracks about $1 \mu$ in length are present to serve as stress concentrators.

\subsection{The Effect of Plastic Flow}

In those crystalline ceramic materials capable of plastic flow, for example, magnesium oxide at room temperature and aluminum oxide at temperatures higher than 1000 ${ }^{\circ} \mathrm{C}$, the shape of a crack tip may be modified as shown in figure 16 . The modification arises from the nucleation and movement of dislocations from the crack tip, a process demonstrated experimentally by Gilman [36]. The process of blunting the crack tip replaces the concentration of elastic stress by a more diffuse distribution of stress associated with dislocations lying along a cylinder whose radius, $R$, depends upon dislocation mobility, see reference [37]. For a surface crack of maximum width h, plastic relaxation can increase the radius of curvature at the tip from the minimum value $\rho=a$ to $\rho=h$ at which value the crack becomes parallel sided. This configuration may be observed readily in soft sodium chloride. Any blunting of the crack makes it necessary to apply a stress greater than that given by eq. (8) which requires that $\rho=a$. Provided that no additional relaxation takes place when a stress is applied, it is better to consider the fracture stress in terms of

$$
\sigma_{f}=\left(\frac{E_{\gamma}}{a}\right)^{1 / 2}\left(\frac{\rho}{c}\right)^{1 / 2},
$$

from which it may be seen that a fully blunted crack only 200 atom spacings wide requires an order of magnitude increase in the fracture stress.

The above discussion of the stress necessary to extend a crack does not take into account important dynamic effects. How rapidly is the applied stress increased from zero to $\sigma_{f}$ ? Is the crack tip advancing at high speed or is it creeping forward? Such questions require consideration for an understanding of the fracture process.

The reason why dynamic effects arise is that the rupture of an atomic or ionic bond is essentially an athermal process whereas plastic flow is thermally activated. The former process depends almost exclusively on the attainment of a critical stress (determined by eqs. (3) or (4)) and is only affected by temperature to a small degree through the corresponding dependence of $\mathrm{E}, \lambda$, and $\mathrm{a}$ on temperature. For an elastic crack whose shape approximates that in figure 15 , the rate at which new bonds are broken, namely the crack velocity, is limited only by the rate at which the applied 
stress is transmitted to the crack tip. A crack cannot travel faster than the velocity of elastic waves in a solid. Stroh [38] has pointed out that the limiting or terminal crack velocity is given by the Rayleigh surface wave velocity. The latter has been calculated by Stoneley [39] to be $2.95 \times 10^{5} \mathrm{~cm} / \mathrm{sec}$ for waves on $\{100\}$ faces of lithium fluoride crystals. This value is in good agreement with a terminal crack velocity of $2 \times 10^{5} \mathrm{~cm} / \mathrm{sec}$ measured experimentally by Gilman et a1. [40] in lithium fluoride monocrystals.

In contrast to the rupture of an atomic bond, plastic flow (embracing the processes of dislocation nucleation, movement, and multiplication) is a thermally activated phenomenon. The important quantity is the plastic strain rate at the crack tip given by $\dot{\varepsilon}=$ bnv . $n$, the number of mobile dislocations per unit area, depends in a complex fashion upon the number of dislocation sources, dislocation multiplication processes, the number of slip systems brought into operation near the crack tip, and most important, on the ability of screw dislocations to cross slip from one slip plane to another. $b$ is the Burgers vector of the moving dislocations and $\bar{v}$, their average velocity, depends exponentially upon both the shear stress $T$ and temperature $T$ [41] through an expression

$$
\bar{v}=v_{0} \exp (A / b \tau k T) \text {, }
$$

where $\mathrm{V}_{\circ}$ and $\mathrm{A}$ are constants. A incorporates factors resisting dislocation motion associated with impurities, point defects and the inherent resistance of the lattice.

Even though the shear stresses just beyond the tip of an elastic crack attain very high values, they must be applied long enough on the same volume of material for stable dislocation loops to form and multiply; in other words the crack must propagate slowly enough for plastic flow to occur at the crack tip. It has been demonstrated [36] that in rock-salt type crystals, dislocation loops may be nucleated on four possible $\{100\}$ planes passing through a region very near to a $\{100\}$ cleavage crack tip as illustrated in figure 16. In a lithium fluoride crystal (typical of those available at the time of investigation) at room temperature Gilman et a1. [41] found a critical crack velocity above which stable dislocation loops were not generated; the value was $6 \times 10^{3} \mathrm{~cm} / \mathrm{sec}$, i.e., 0.01 times the terminal speed. At velocities just below this value, cleavage cracks left a trail of dislocation half loops in the fracture surfaces. Under these conditions, the steady state radius of curvature of the crack tip during propagation was therefore slightly greater than the interionic distance. At a smaller average velocity of $3 \times 10^{3} \mathrm{~cm} / \mathrm{sec}$, the crack propagation was found to be unstable. The velocity oscillated between high values to very small values approaching zero. At each point where the crack slowed down, a large number of dislocations were nucleated leading to a substantial blunting of the crack tip (leaving steps in the fracture surface) and a corresponding increase in propagation stress. It is interesting to note that those lithium fluoride crystals which are available commercially today contain less divalent cation impurities than those investigated above and are much more difficult to cleave at room temperature without introducing many dislocations. This increased resistance to cleavage is a direct consequence of the increased dislocation mobility in the higher purity material.

An increase in temperature has a qualitatively similar effect on crack propagation as a decrease in crack velocity. Just as there is a critical crack velocity below which propagation becomes unstable, so there is a critical or transition temperature above which crack propagation becomes difficult [42]. The strain rate applied during crack propagation controls the transition temperature above which the material becomes notch insensitive. For example, at low strain rates of $10^{-2} / \mathrm{min}$ one cannot cleave silver chloride monocrystals at temperatures as $10 \mathrm{w}$ as $0.1 \mathrm{~T}_{\mathrm{m}}$ (1iquid nitrogen temperature) but at high strain rates of $10^{3} / \mathrm{min}$, crystals cleavage at $0.4 \mathrm{~T}_{\mathrm{m}}$. A similar transition has been observed in sodium chloride [20] at a strain rate of $10^{2} / \mathrm{min}$ but it occurs at a much higher homologous temperature, namely, $0.95 \mathrm{~T}_{\mathrm{m}}$. Lithium fluoride and magnesium oxide behave similarly to sodium chloride.

The improvement in resistance to cleavage which accompanies an increase in temperature is certainly a reflection of increased dislocation mobility. However, this explanation is not really adequate. There is reason to believe that in rock-salt type crystals, an explanation must also include the corresponding effect of temperature on the relative ease with which dislocations move on, and cross slip between, slip planes other than those of the $\{110\}$ type, namely $\{100\}$ and $\{111\}$.

Silver chloride is capable of deforming readily on many slip systems simultaneously (yielding "wavy glide" traces) at very low homologous temperatures, $0.1 \mathrm{~T}_{\mathrm{m}}$, whereas in lithium fluoride it is not until $0.4 \mathrm{~T}_{\mathrm{m}}$ is reached that the possibility of gross cross slip between $\{110\}$ and $\{100\}$ becomes significant. Figure 17 shows that in the range 300 to $400{ }^{\circ} \mathrm{C}$, there is a rapid decrease in the ratio of the stress re- 
quired for glide on $\{001\}$ planes to that required for $\{110\}$ glide [43]. The important feature is that the ability to slip on many slip systems endows a solid with more (plastic) degrees of freedom to relax shear stresses at the tip of a running crack. It should also be noted that cross slip is probably important in crack nucleation, in that the increased ability to cross slip should increase the possibility of stress relaxation at the ends of blocked glide bands and other plastically induced stress concentrations.

The value of $\rho$, in eq. (9), which determines the applied stress necessary for crack propagation, will have an instantaneous value which is determined by the crack velocity, temperature and the magnitude and extent of the shear stress near the crack tip. Unfortunately it is difficult to determine the actual shape of a crack and therefore the magnitude of the stress at the tip. This problem is often avoided by regarding plastic flow as a means of absorbing energy, and to include it as an extra term in the Griffith equation as Orowan [44] suggested (even though we cannot predict the amount). In this way, the equation is modified by replacing the surface energy term $\gamma$ by $\mathbf{P}$, a term which is the sum of $\gamma$, and the plastic work done per unit area of fracture surface, D. However, it should be remembered that for propagation to continue, a stress of the order of $(\mathrm{E} \gamma / \mathrm{a})^{\frac{1}{2}}$ must be applied at the crack tip regardless of the shape of the edge of the crack. It may be more straightforward, therefore, to regard the term $P$, not as $\gamma+D$, but as $\gamma \cdot 0 / a$, in which case the modified Griffith equation is identical to eq. (9). When sufficient plastic flow accompanies crack propagation to yield a value of $p \simeq 100 \mathrm{a}$, so the effective fracture surface energy will be $10^{2} \times \gamma$.

\subsection{Fracture Surface Topography}

During propagation, there is a tendency for a crack to spread on more than one plane. The boundaries between neighboring levels of the fracture surface define steps which always lie perpendicular to the advancing crack front. Cleavage steps, sometimes called "river patterns" or tear lines, provide an excellent means of determining the direction of crack propagation so that one can often find where a crack has started.

These are several reasons why steps form:

1. At high crack velocities, there is rotation of the maximum tension stress at the tip from a direction perpendicular to the plane of the crack [45]. This rotation has the effect of causing the crack to deviate from a flat plane surface. When the deviation varies from point to point along a curved crack front, steps are generated. Smekal [46] has pointed out that lines or steps are formed also by the interaction of the moving crack edge and elastic waves originating from secondary sources. Steps of this kind ("Wallner lines" [47]) are a common feature of the conchoidal fracture of brittle materials in which the cohesive strength is essentially isotropic. If there is a pronounced anisotropy of cohesive strength, cracks are more constrained to lie parallel to certain crystallographic planes; nevertheless, steps can still form by the following second mechanism.

2. It will be recalled that a screw dislocation is one in which the lattice planes normal to it constitute a helical ramp centered about the dislocation. When a crack intersects a screw dislocation, the plane of the crack is offset by an amount equal to the Burgers vector of the dislocation. Gilman [48] correlated the formation of steps with screw dislocations present in (a) twist boundaries (made up of crossed grids of screws), (b) glide bands formed prior to crack formation, and (c) dislocations formed just ahead of the crack tip during propagation.

3. In a polycrystalline material the opportunity for step formation is magnified. Where the orientation across the boundary between two adjoining grains changes very little, cleavage steps can originate there in a manner similar to that associated with simple twist boundaries or because the crack crosses the boundary by the nucleation of a series of new cracks at many points along the boundary. The presence of large angle boundaries increases the probability of a crack spreading by the successive nucleation and joining together of new cracks formed ahead of the principal crack front. Cracks formed in this way will rarely be on the same plane as the main crack so that very large steps or tear lines are likely to form.

4. Steps can form as a consequence of the intersection of a crack with inclusions, a second phase or large pores.

The ability of a glide band to block the growth of a small crack is related to the formation of a large number of steps by mechanism 2 as the crack crosses the glide band. To calculate the increase in the stress necessary to extend a crack due to step formation one, in principle, could analyse the problem in terms of the change 
in the shape of the crack front. However, such an analysis is clearly very difficult. It is more convenient to look upon step formation as an increase in fracture surface area per unit length of crack. In other words, the effective fracture surface energy is increased by the creation of steps. It has been estimated [37] that in the absence of plastic relaxation during crack growth, $\gamma$ can increase to a maximum value of $2 \gamma$. If the crack velocity is low enough or the temperature high enough, the threads of material connecting the different levels of fracture surface may rupture by plastic shearing so that $\Delta \gamma$ will be greater than $\gamma$. Figure 18 illustrates the difference in appearance of cleavage steps in silver chloride monocrystal produced at two different temperatures. Those formed at the higher temperature are discontinuous and severely distorted.

\section{Summary}

A description has been given of the introduction of cracks into solids by mechanical damage and by plastic flow processes. In the harder materials, the strength is controlled primarily by the state of the surface, in other words, by the number, distribution and size of the pre-existing cracks. In soft crystals, the strength is still influenced by the state of the surface but it is dependent also on the stress to initiate yielding and on the distribution of slip. No attempt has been made to set up a comprehensive fracture criterion because it is premature to do so - even though we probably know more about the details of fracture processes in ionic materials than we do in metals.

Although detailed consideration has been given to the nucleation of cracks in magnesium oxide-type crystals as a result of plastic deformation, it should not be overlooked, that from a pragmatic point of view, the most important source of cracks are those introduced by mechanical contact or abrasion just as in the case of sapphire or titanium carbide. Both classes of ionic materials presently available are notch sensitive to a degree far in excess of most metals. The magnesium oxide which is presently available suffers from the fact that dislocations can move and interact to form cracks at low stress levels, yet dislocation mobility on a sufficient number of slip systems is not adequate enough to blunt the tip of a running crack.

The author thanks Dr. A. J. McEvily for helpful discussions.

\section{References}

[1] J.Frenke1, Z. Phys. 37, 572 (1926).

「2] E. Orowan, Rept. Prog. Phys. 12, 185 $(1948-1949)$.

[3] J. J. Gilman, Fracture, p. 193, M.I.T. Tech. Press and John wiley \& Sons (1959).

[4] J. J. Gilman, J. App1. Phys. 31, 2208 (1960).

[5] J. E. Lennard-Jones and P. A. Taylor, Proc. Roy. Soc. (London) A109, 476 (1925).

[6] W. Weibull, Proc. Ingen. Vetenskaps. Acad. 151, No. 153 (1939).

[7] T. L. Johnston, R. J. Stokes, and C. H. Li, Phil. Mag. I, 23 (1962).

[8] A. R. C. Westwood, Phil. Mag. 6,195 (1961).

[9] W. Class, E. S. Machlin, and G. T. Murray, Trans. A.I.M.E. 221, 769 (1961).

[10] R. J. Stokes, T. L. Johnston, and C. H. Li, Trans. A.I.M.E. 218, 655 $(1960)$.
[11]
N. A. Brilliantov and I. W. Obreimov, Phys. Z. Sowjetunion 12, 7 (1937).

A. Taylor and P. L. Pratt, Phil. Mag. 3, 1051 (1958).

R. J. Stokes, T. L. Johnston, and C. H. Li, J. Appl. Phys. 33,62 (1962).

B. H. Kear, A. Taylor, and P. L. Pratt, Phil. Mag. 4, 665 (1959).

R. J. Stokes, T. L. Johnston, and C. H. Li, Phil. Mag. 4, 920 (1959).

F. J. P. Clarke and R. A. J. Sambell, Phil. Mag. $\underline{5}, 697$ (1960).

J. Washburn, A. E. Gorum, and E. R. Parker, Trans. A.I.M.E. 215,230 (1959).

A. D. Wapham and M. J. Makin, Phil. Mag. $\underline{5}, 237$ (1960).

M. A. Adams and G. T. Murray, to be published.

T. L. Johnston, R. J. Stokes, and C. H. Li, Strengthening Mechanisms in Solids, A.S.M. Seminar (1960). 
[21] N. M. Melankholin and V. R. Regel, Soviet Phys. J. Eksp. Teoret. Fiz. $\underline{2}, 696$ (1956).

[22] R. J. Stokes, T. L. Johnston, and C. H. Li, Phil. Mag. 6, 9 (1961).

[23] R. J. Stokes, Honeywel1 Research Center Report (1962).

[24] J. J. Gilman, J. App1. Phys. 30, 1584 (1959).

[25] C. Zener, Fracturing of Metals, A.S.M., [39] p. $3(1948)$.

[26] J. S. Koehler, Phys. Rev. 85, 480 (1952) [40]

[27] J. D. Eshelby, F. C. Frank, and F. R. N. Nabarro, Phil. Mag. 42, 351 (1951).

[28] R. J. Stokes and C. H. Li, Honeywel1 Research Center O.N.R. 15th Tech. Rept. (1962).

[29] A. S. Argon and E. Orowan, Nature 192, 447 (1961).

[30] G. D. Miles and F. J. P. Clarke, Phil. Mag. $\underline{6}, 1449$ (1961).

[31] A. Joffe, M. W. Kirpitschewa, and M. A. Lewitsky, Z. Phys. 22, 286 (1924).

[32] R. J. Charles, Prog. Ceram. Sci. 1, I (Pergamon Press, 1961).

[33] C. E. Inglis, Trans. Inst. Naval Arch. 55, 219 (1913).

「34] H. A. Elliot, Proc. Phys. Soc. 59, 208 (1947).

\section{POTENTIAL}

ENERGY

$(u)_{r}$

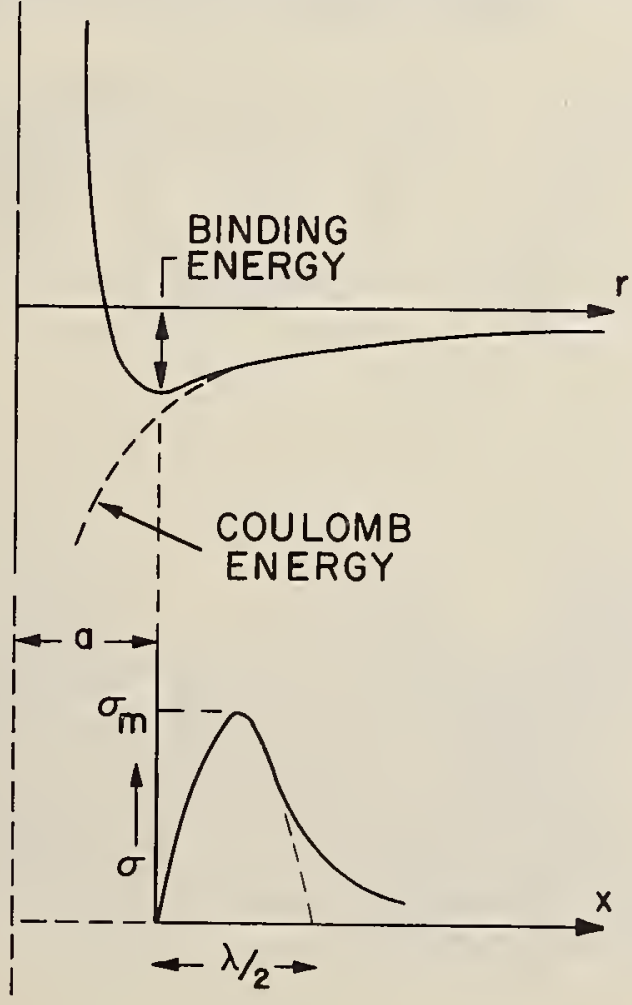

[35] A. A. Griffith, Phil. Trans. (London) A221, 163 (1921).

[36] J. J. Gilman, Trans. A.I.M.E. 209, 449 (1957).

[37] J. Friedel, Fracture, p. 498, M.I.T. Tech. Press and John Wiley \& Sons (1959).

[38] A. N. Stroh, Advance Phys. 6, 418 (1957).

R. Stoneley, Proc. Roy. Soc. 232, 447 (1955).

J. J. Gilman, C. Knudsen, and W. Walsh, J. Appl. Phys. 29,601 (1958).

[41] J. J. Gilman, Prog. Ceram. Sci. 1, 146 (Pergamon Press, 1961).

[42] T. L. Johnston, R. J. Stokes, and C. H. Li, Phil. Mag. 4, 1316 (1959).

[43] J. J. Gilman, Acta Met. ㄱ, 608 (1959).

[44] E. Orowan, Proc. Symp. Fatigue and Fracture of Metals, p. 139, M.I.T. Tech. Press and John Wiley \& Sons (1950).

[45]

E. H. Yoffe, Phil. Mag. 42, 739 (1951).

[46] A. Smekal, Ergeb. oxakt, Naturw. $\underline{15}, 196(1936)$.

[47] A. Wallner, Z. Phys. 114, 368 (1939).

[48] J. J. Gilman, Trans. A.I.M.E. 212, 310 (1958).
FIGURE 1. Schematic representation of (a) the change in potential energy and (b) the force required, to separate adjacent planes in an ionic crystal. 

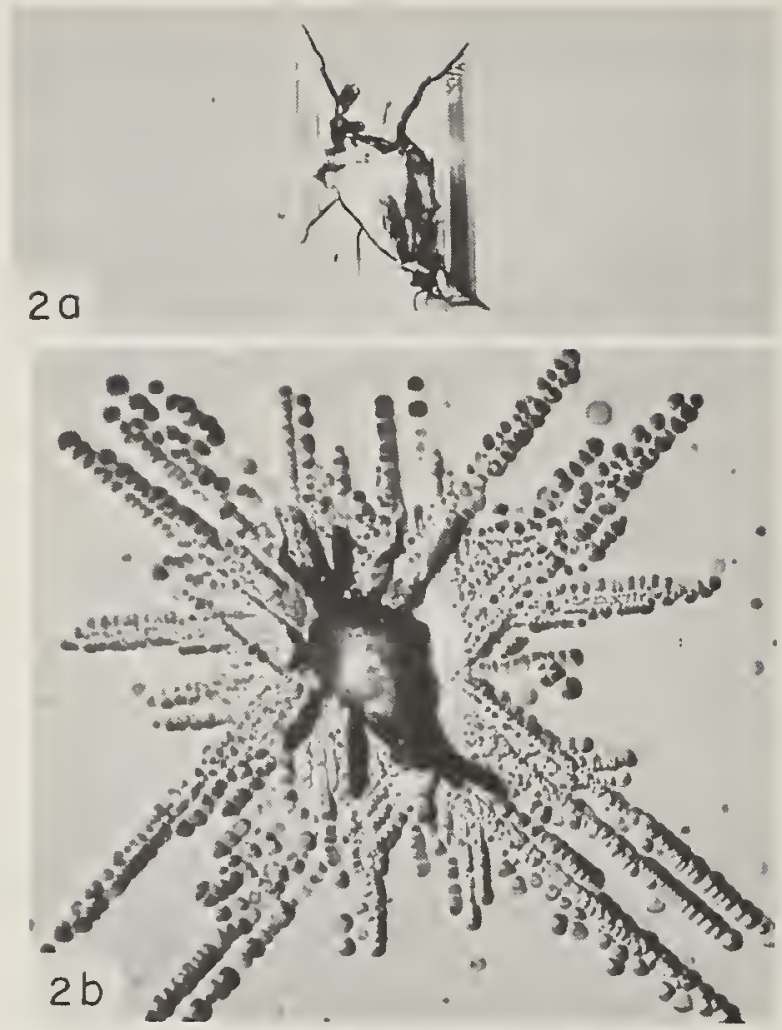

FIGURE 2(a). Damage produced on the surface of a polished magnesium oxide crystal by the impact of a silicon carbide particle $1 / 16$ in. diameter dropped from a height 2 in. ( $X$ 562).

(b) Dislocation arrays associated with crater shown in figure 2(a) (X 562).

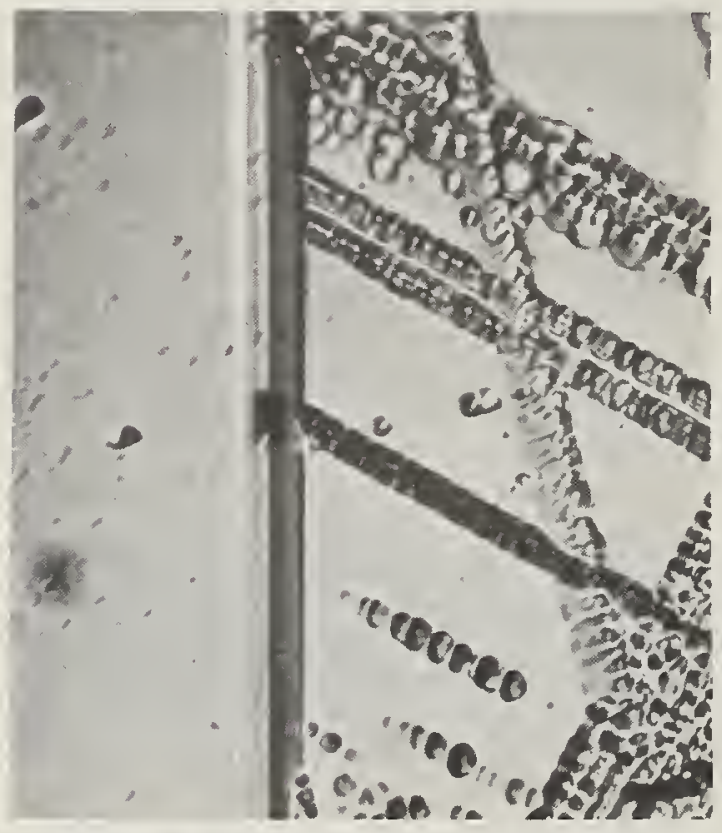

FIGURE 4. Intergranular crack formed at the tip of a blocked glide in magnesium oxide (X 562). (After Johnston et a1.) [20].

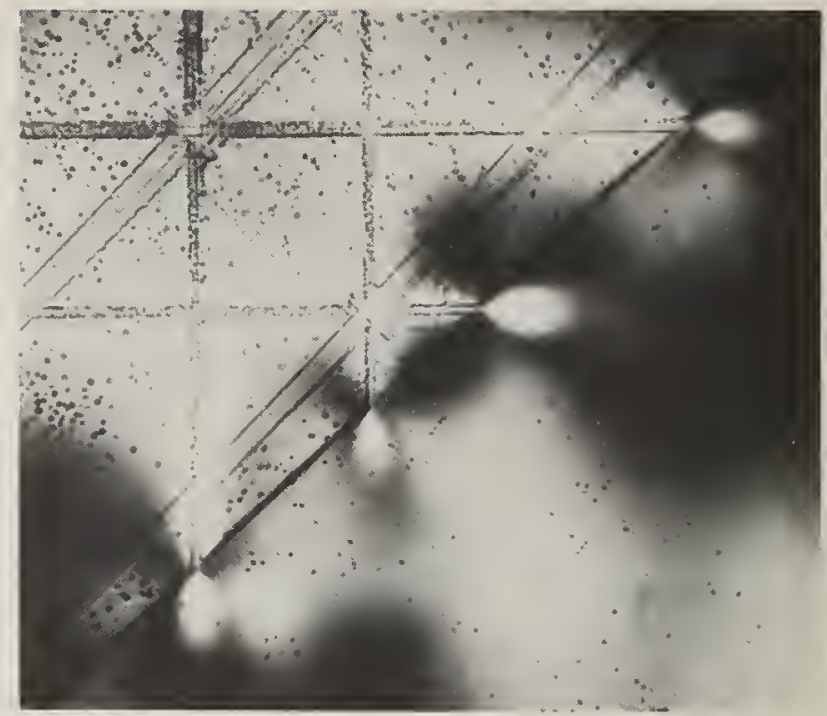

FIGURE 3. Stress concentrations revealed by stress birefringence associated with glide bands blocked at a grain boundary in magnesium oxide (X 75).

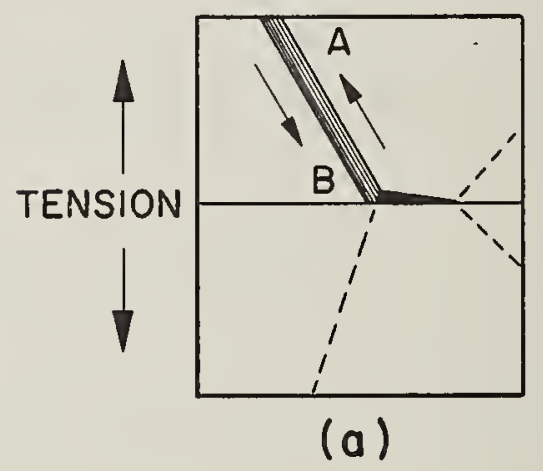

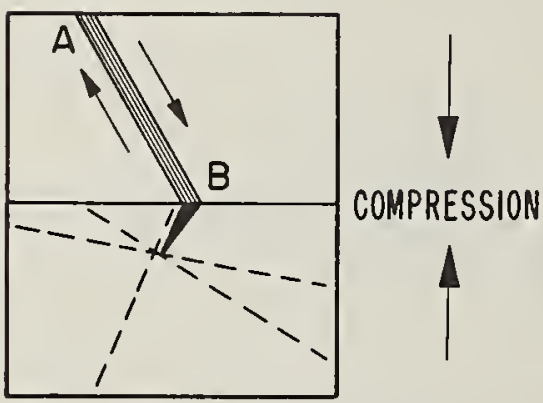

(b)

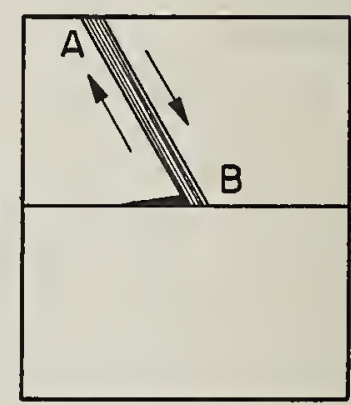

(c)
FIGURE 5. Schematic diagrams showing observed crack positions with respect to the nucleating glide band $A B$. Dotted lines are secondary glide bands generated by the cracks. Configuration (a) applies to medium and large angle boundaries, configurations (b) and (c) apply to medium and large angle boundaries respectively. 
FIGURE 6. Generation of a grain boundary crack through the interaction of two glide bands $A B$ and $A^{\prime} B{ }^{\prime}$.

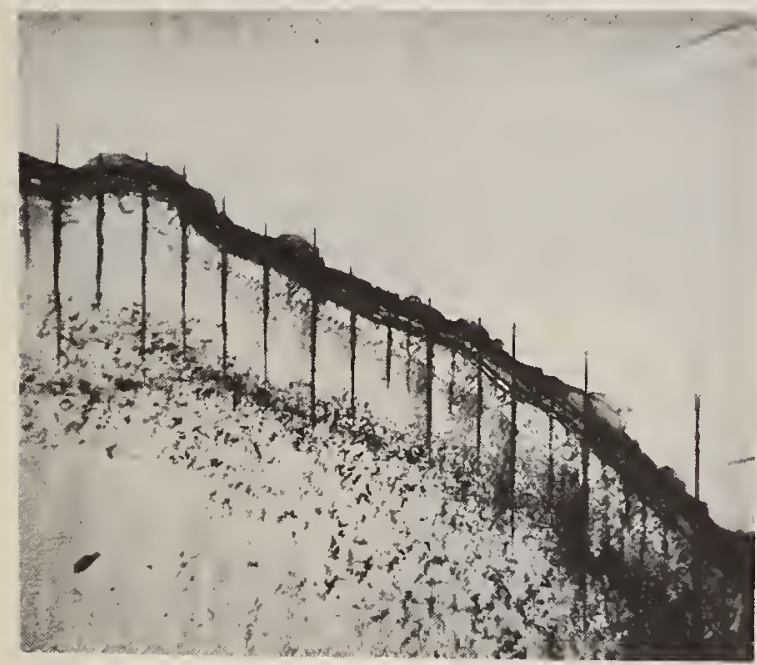

FIGURE 7. Microcracks formed in the tension surface of a bent sodium chloride crystal. Cracks are centered on rim of deposit (X 37).

(After Stokes et al.) [10].
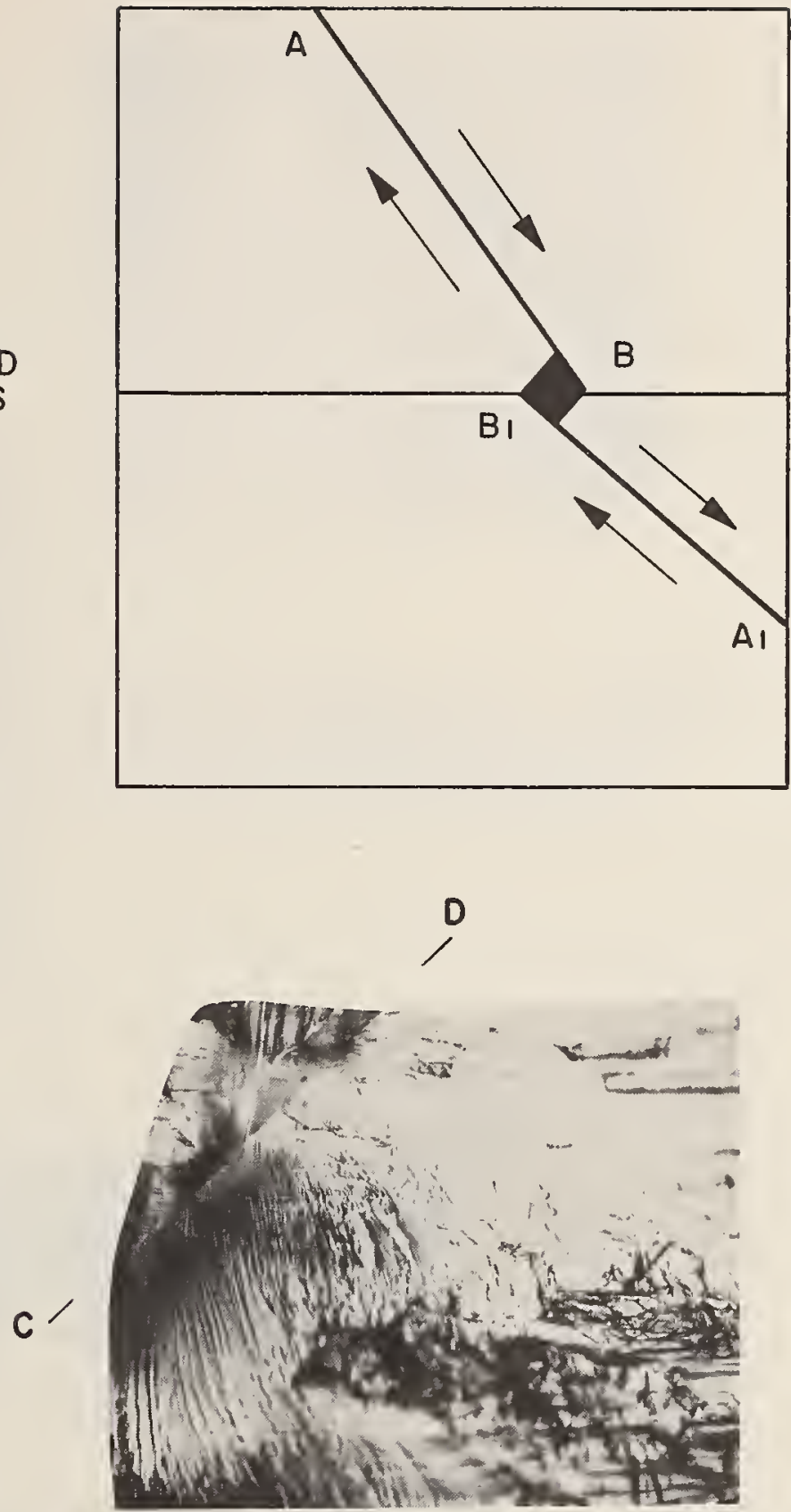

FIGURE 8. Fracture surface of a sodium chloride crystal bent at liquid nitrogen temperature in which kink had formed across a corner on the tension side (X 52). (After Stokes et al.)[13].

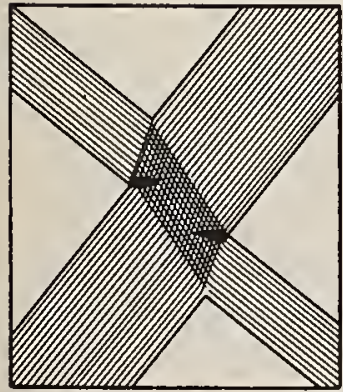

(a)

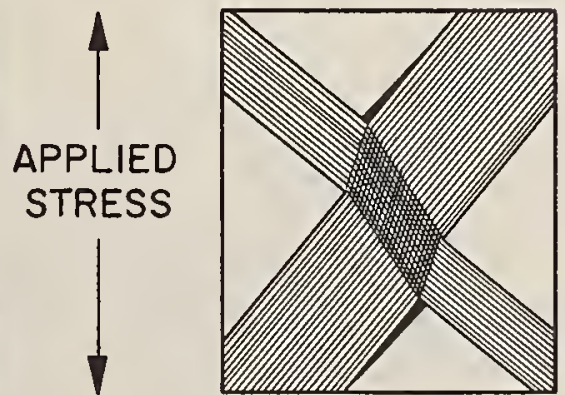

(b)
FIGURE 9. Schematic diagram showing the formation of (a) (100) and (b) (110) cracks respectively at the intersection of $\{110\}$ glide bands. 


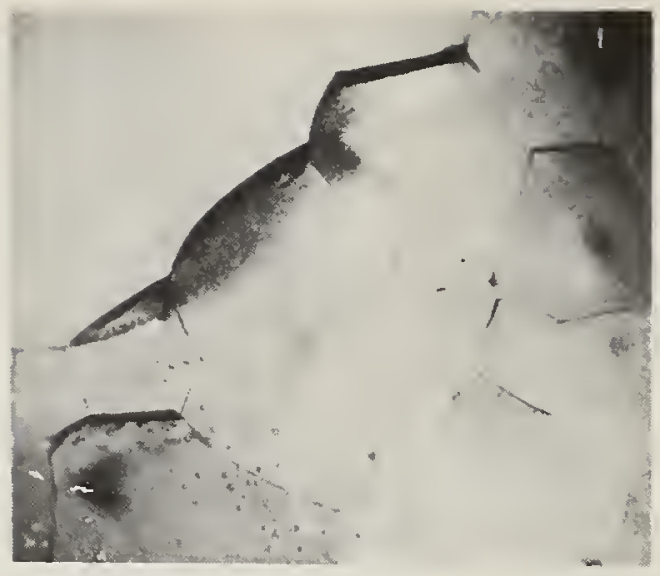

FIGURE 10. Intergranular cracking produced by grain boundary sliding in the tension surface of a lithium fluoride polycrystalline rod, loaded in simple bending at $400{ }^{\circ} \mathrm{C}$ at a nominal maximum strain rate of $10^{-1} / \mathrm{sec}(X 75)$.

(After Johnston et a1.) [20].

FIGURE 11. Stabilization of $\{110\}$ microcracks at slip band intersections in magnesium oxide (X 112). (After Stokes et a1.)[15].
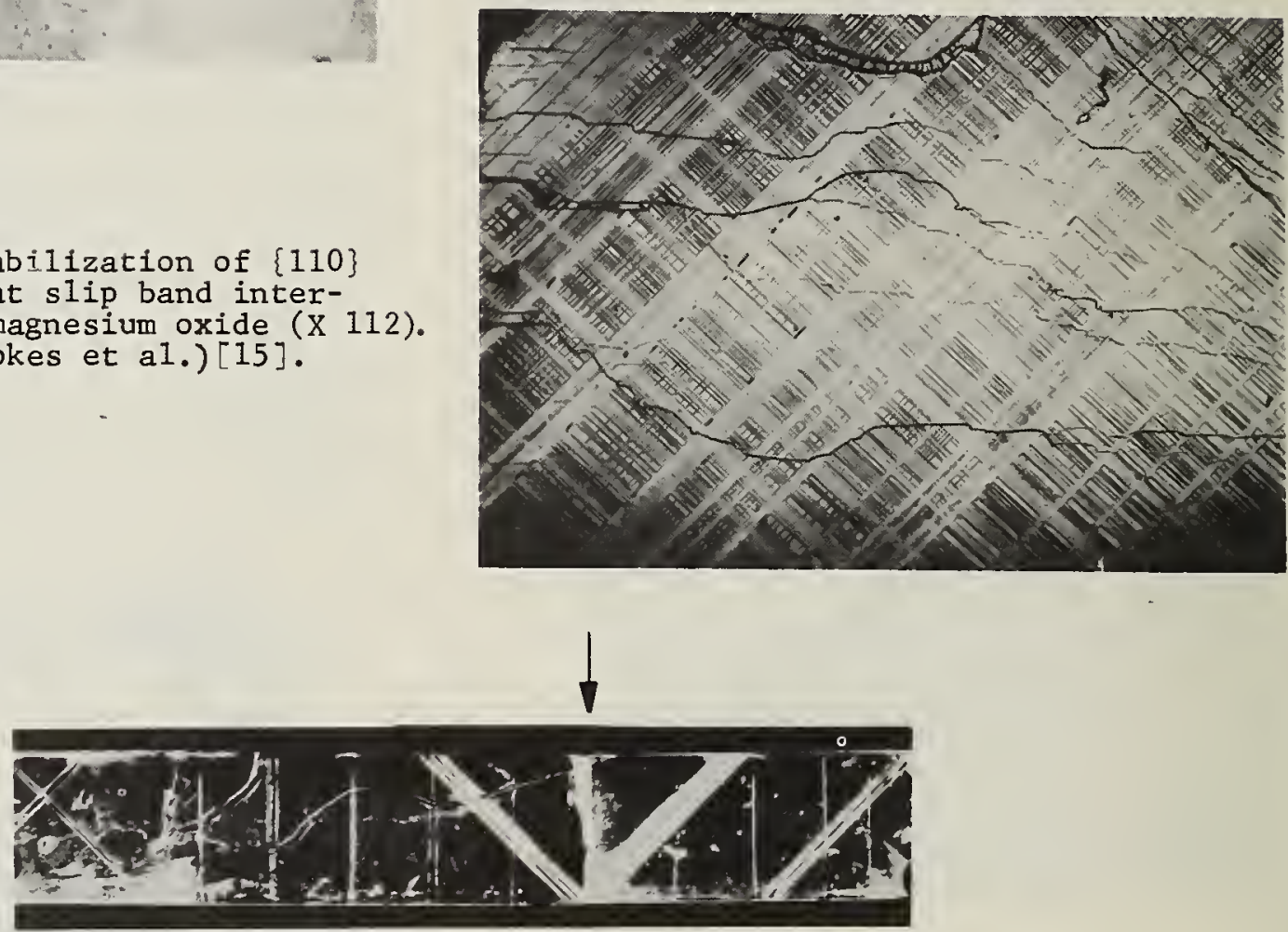

FRACTURE ORIGIN

FIGURE 12. Photomicrograph showing the distribution of slip after fracture in a magnesium oxide crystal (X 7 ).

(After Stokes et a1.)[22].

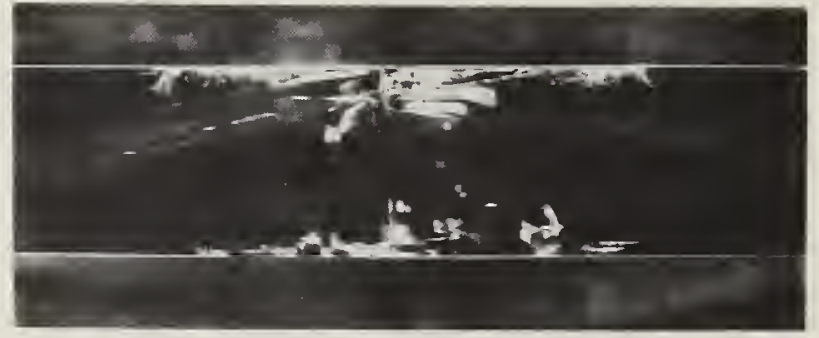

FIGURE 13. Tension surface of a magnesium oxide crystal fractured in bending; slip was very finely spaced. Note longitudinal cracks at specimen corners ( $X 4$ ). (After Stokes et a1.)[22]. 


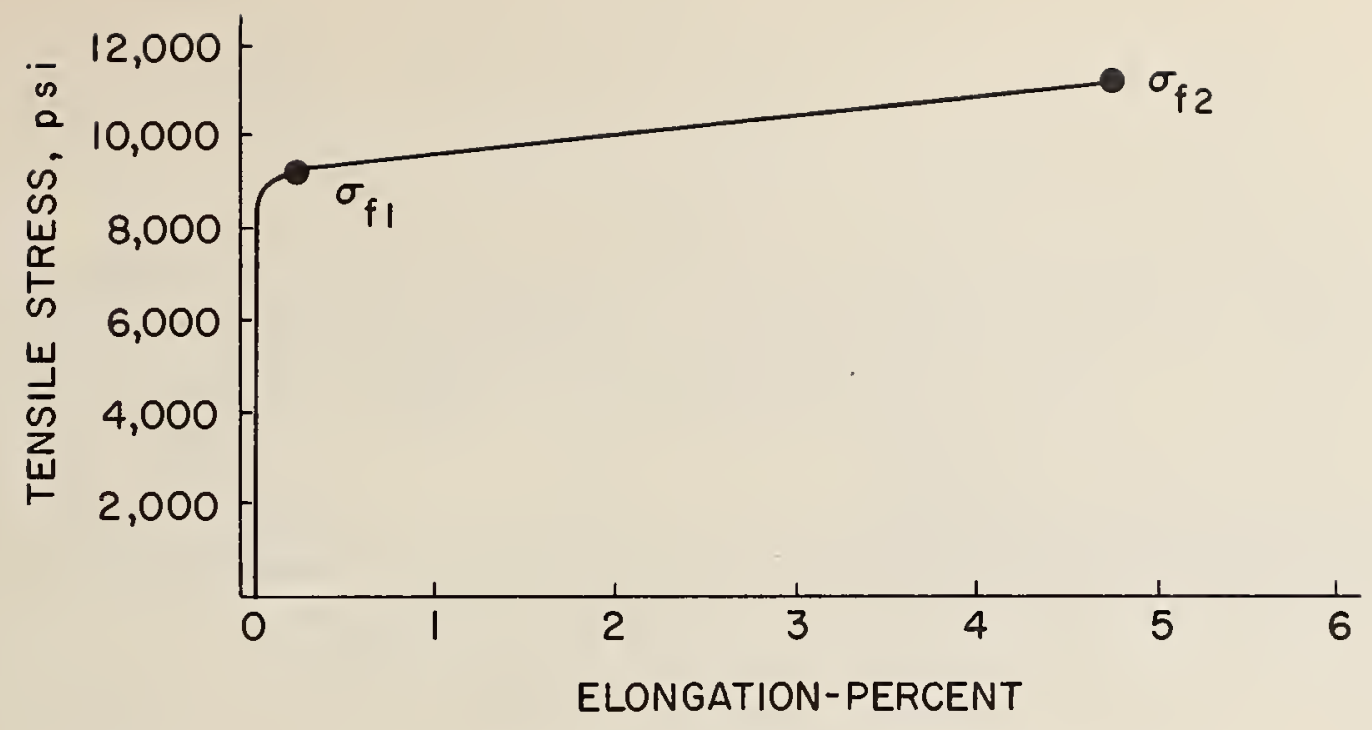

FIGURE 14. Typical stress-elongation curve for a chemically polished magnesium oxide crystal loaded at room temperature. $\sigma_{f}$ and $\sigma_{f_{2}}$ are the fracture stresses of specimens in which very few, ${ }^{f_{2}}$ and a large number of slip bands, were formed repective$1 y$ at the onset of yielding.

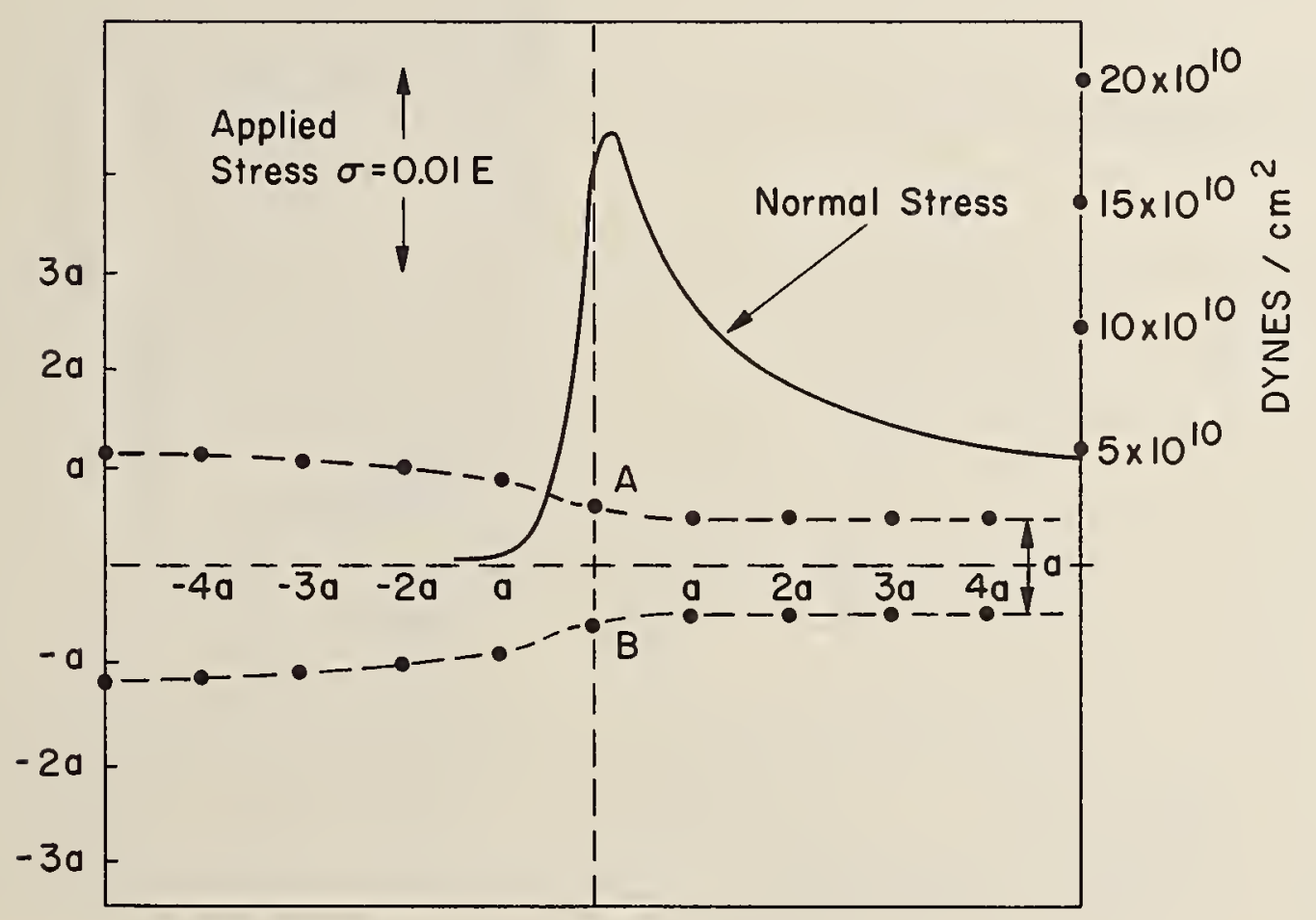

FIGURE 15. Atomic positions and distribution of normal stress at the edge of a crack of length $2 \mathrm{c}=1000 \mathrm{a}$ for an applied stress $\sigma=0.01 \mathrm{E}$ where $\mathrm{E}=10^{12}$ dynes $/ \mathrm{cm}^{2}$. 


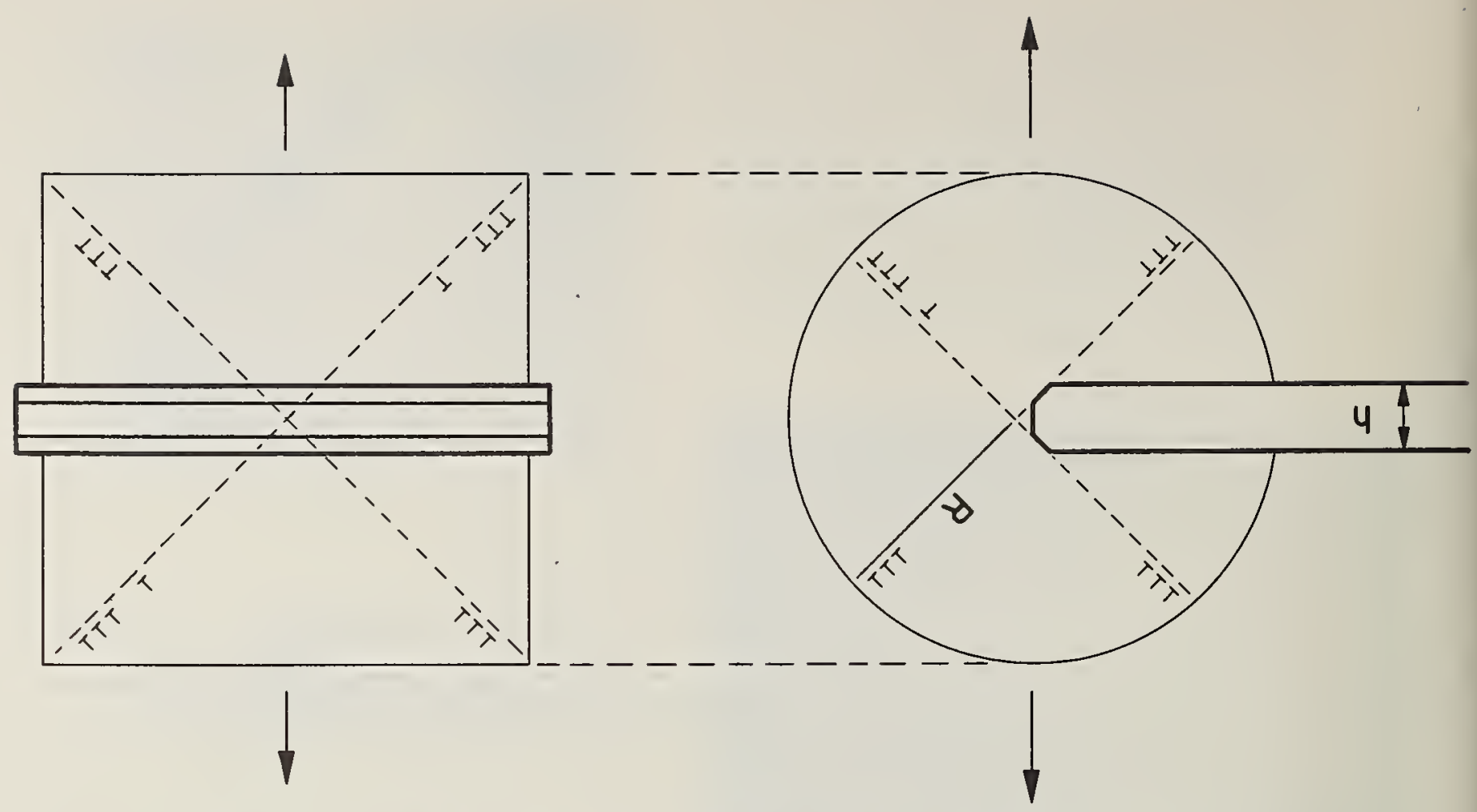

FIGURE 16. The tip of a (100) cleavage crack modified by the nucleation of dislocations from the tip and their movement on four possible $\{110\}$ glide planes. The dislocations lie along a cylinder of radius $R$.
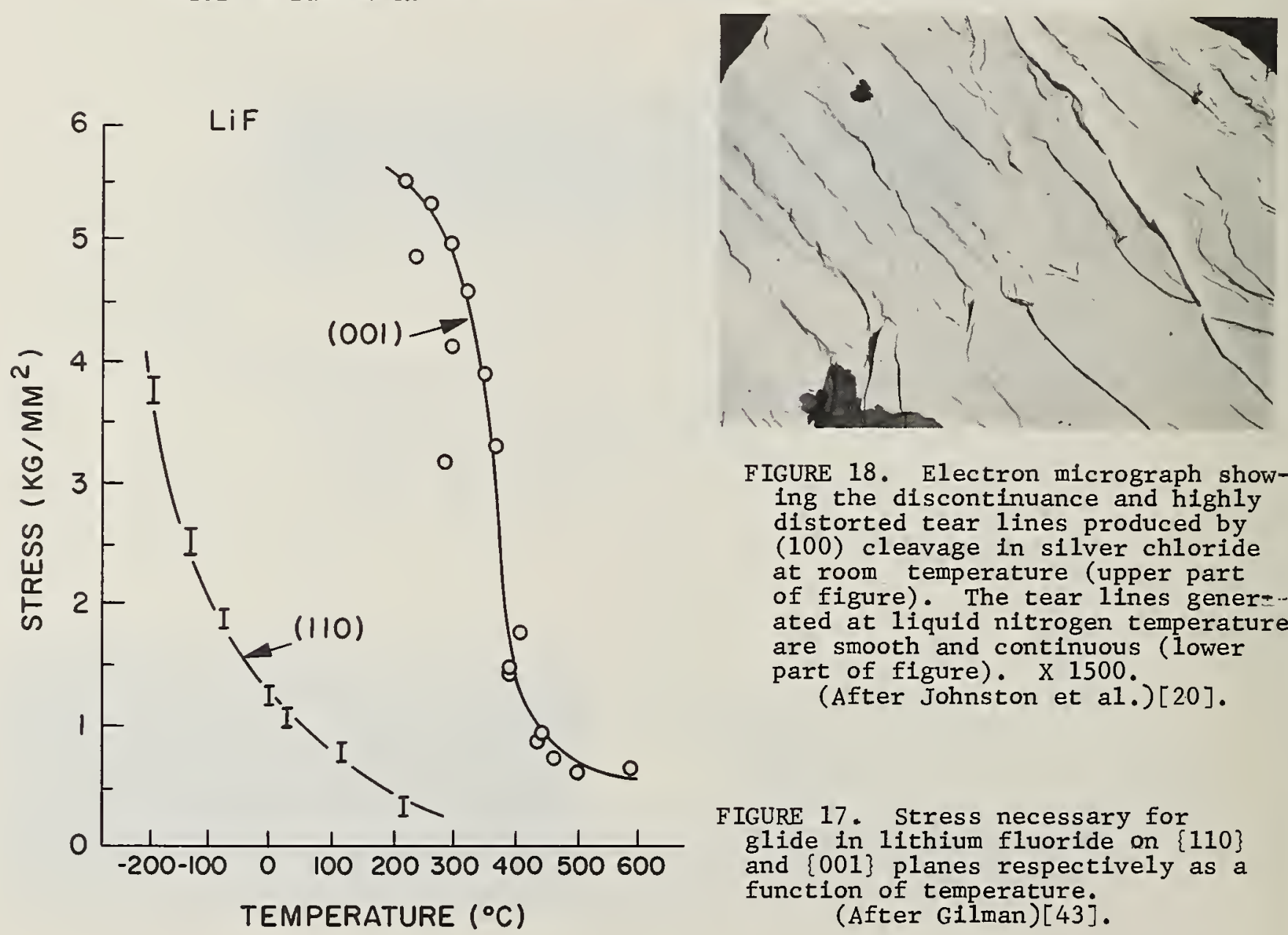

FIGURE 18. Electron micrograph showing the discontinuance and highly distorted tear lines produced by (100) cleavage in silver chloride at room temperature (upper part of figure). The tear lines gener:ated at liquid nitrogen temperature are smooth and continuous (lower part of figure). X 1500.

(After Johnston et a1.)[20].

FIGURE 17. Stress necessary for glide in lithium fluoride on $\{110\}$ and $\{001\}$ planes respectively as a function of temperature. (After Gilman)[43]. 
John J. Gilman

Division of Engineering, Brown University

\section{Introduction}

Most scientific interpretations of phenomena pass through three periods of development. The first is a period of conceptual elaboration of the initial idea to improve its clarity and determine its scope of applicability. Next is a period of probation during which the validity of the interpretation is tested. Finally, if it proves to be valid, enough confidence is generated for the idea to be used as a basis for newer interpretations, and for technological inventions.

The concept of crystal dislocations has recently reached the end of the second stage in its development as an idea, and is currently passing into the third stage. Although studies of dislocations were initially devoted to metals, it seems likely that some of the most important applications of our knowledge of them will be applied to ceramic crystals. Therefore, this symposium seems to be an appropriate place to discuss what some of the technological consequences of dislocation physics might be. If these consequences prove to be only partially as important as those of electron physics, they will cause major technological changes.

The further we get from 1934 - the birthyear of crystal dislocations ${ }^{1}$ - the more simple the essence of a dislocation line seems. At the same time, the consequences of the idea have become more and more subtle. This accounts, at least in part, for the relatively slow maturation of the subject.

Before the conception of crystal dislocations, people had tried to treat plastic flow as a homogeneous process. This point-of-view was fruitless because the process is, in fact, a highly heterogeneous one. Orowan, Polanyi, and Taylor postulated that it is heterogeneous even on an atomic scale. That is, the shearing action that results in plastic deformation does not occur homogeneously everywhere on a glide plane. Instead, it starts at some special place and gradually propagates over the remainder of the plane. The boundary of the area over which gliding has occurred is the dislocation line.

As the glided area spreads, displacement of the top of the crystal relative to the bottom increases in proportion to the growth of the glided area. The maximum value that the displacement reaches is $b$, the unit glide displacement. Thus the displacement, $\delta$, at any time is:

$$
\delta=\mathrm{b}\left(\mathrm{A} / \mathrm{A}_{\mathrm{O}}\right),
$$

where $A_{0}$ is the area of the entire glide plane. Several dislocations usually move at the same time during plastic deformation so the total displacement, $\Delta$, is the sum of the individual ones:

$$
\Delta=\sum_{i}^{n} \delta_{i}=\frac{b}{A_{0}} \sum_{i}^{n} A_{i}
$$

replacing the sum by $\mathrm{n}$ times the average value, $\overline{\mathrm{A}}$ :

$$
\Delta=\frac{\mathrm{nb} \overline{\mathrm{A}}}{\mathrm{A}_{\mathrm{O}}}
$$

then, if $\mathrm{h}$ is the height of the crystal, the plastic strain is:

$$
\gamma=\frac{\Delta}{h}=\frac{n b \bar{A}}{h w I}
$$

where $A_{0}=w L(w=$ width; $L=$ length of crystal). But the area density of dislocations, $\mathrm{o}$, is equal to $\mathrm{n} / \mathrm{hL}$, and, letting $\mathrm{w}$ be a unit width:

${ }^{1}$ There are a variety of priority claims, but this year marks the appearance of clear expositions of the idea in the public literature by Orowan, Polanyi, and Taylor. 


$$
\gamma=\mathrm{b} \rho \bar{A}
$$

The average area swept out by dislocations, $\bar{A}$, is difficult to determine experimenta11y, so the strain-rate is considered rather than the strain. Taking the time derivative ${ }^{2}$ :

$$
\frac{\mathrm{d} \gamma}{\mathrm{dt}}=\mathrm{b} \circ \frac{\mathrm{d} \overline{\mathrm{A}}}{\mathrm{dt}}
$$

This is the most fundamental relationship of the dislocation theory of plastic deformation. It has been verified experimentally in considerable detail (see, e.g., Gilman and Johnston, 1962), so that one can consider that the theory is not only plausible, but also unique in the sense that no additional mechanisms of plastic flow need to be postulated.

The firm establishment of dislocation theory has filled in the last remaining large gap in fundamental understanding of the mechanical behavior of solids. The elastic behavior has been understood for some time both macroscopically (Hooke's Law and its consequences), and microscopically (Born's ionic crystal theory, Wigner-Seitz theory of metals). The Griffith crack theory forms the basis of understanding fracture phenomena. Thus a11 of the elements of mechanical science now exist, and the time has come to combine them so as to produce exceptionally strong materials, and make other advances.

When steel became an inexpensive article of commerce in the 1860 's, it was an order of magnitude stronger than anything else at that time (150,000 psi as compared to 15,000 psi for wrought iron). It is not surprising, therefore, that it revolutionized every industry that it touched. Materials with strengths an order of magnitude greater than the best steel would certainly have major consequences at the present time. This would require strengths to increase into the millions of pounds per square inch range.

Just as the advent of steel required new manufacturing and structural design concepts, a new jump in mechanical strength will require changes. A higher level of sophistication will be needed in order to have precisely controlled manufacturing operations, and adequate designs.

The remainder of this discussion will be concerned with the problems associated with ultra-strong materials. Some questions are: what is the best chemical composition for ultra-strength? What would be the best internal structure? How must the surface be prepared? What manufacturing and design difficulties can be expected to arise? If an ultra-strong material existed, what devices really require it? Sensible, although perhaps not final, answers to these questions can be obtained from modern knowledge of the mechanical behavior of solids and some intuitive guesses.

\section{The Limit of Cohesive Strength}

Materials that already exist, such as steel, have great strength. Therefore, the room that is available for improvement is not large. In order to produce something that is substantially stronger than existing materials, it will be necessary to carefully optimize all possible factors. The primary thing to be optimized is the intrinsic cohesion of the material: First, because it would be foolish to try to produce ultra-strength in a substance whose atoms are not tightly bound; and second, because one should be aware of the absolute upper limit on strength set by the laws of chemical binding. This limit is only one or two orders of magnitude higher than the strength of steel. Thus, one must work as near the upper limit as possible.

In principle, one might calculate cohesive stresses from the theory of chemical binding. However, this is not a practical procedure because such calculations would be exceedingly tedious and not highly reliable; especially for anisotropic crystals. A better procedure is to find relations between measurable physical properties and cohesive stresses. By using properties that are anisotropic, one can obtain cohesive stresses for various crystallographic directions.

Four methods for determining cohesive stresses are illustrated in figure 1 . The first (figure la) takes the cohesive stress to be a certain fraction of the Young's modulus, $E_{h k 1}$, in a particular crystallographic direction. It is useful as a rule of

\footnotetext{
${ }^{2}$ Note that the term, $b \bar{A} \frac{d \rho}{d t}=0$, because the creation of a new dislocation is a discontinuous process.
} 
thumb, but rather crude. Usually the cohesive stress, $\sigma_{c}$, is taken to equal 10 percent of the modulus $(M=10)$.

The second method (figure 1B) uses some additional information to yield an improved estimate. Common sense tells us that the net stress between the atoms in a crystal must be zero in the absence of applied stresses; must rise to some maximum value as the atoms are pulled apart; and then must fall to zero again as the crystal breaks into two or more pieces. The rise and fall of the stress is often represented by one-half of a sine curve (figure 1B). For small increases in the distance between atoms, the stress will obey Hooke's Law; $\sigma_{\mathrm{hkl}}=\mathrm{E}_{\mathrm{hkl}}\left(\frac{\mathrm{y}}{\mathrm{y}_{0}}\right)$; so the cohesive stress will be: $\sigma_{c}=\frac{E_{h k 1}}{\pi}\left(\frac{a}{y_{o}}\right)$; where $y_{o}$ is the initial separation of the atoms, and $a$ is the "range" of interatomic forces.

A third method (figure 1C) relates $\sigma$ to the surface energy of the planes across which a crystal is pulled apart. A somewhat improved interatomic force law is used which expresses the fact that the force is not expected to suddenly drop to zero as in figure 1B. Using the initial slope condition, the constant $A$ is found to equal $\mathrm{E} / \mathrm{y}_{\mathrm{O}}$. The constant, $\mathrm{a}$, is determined by the surface energy, $\gamma_{\mathrm{hkl}}$, since:

$$
\gamma=1 / 2 \int_{0}^{\infty} \sigma d y=\frac{A}{2 a^{2}}
$$

where the integral is the work required to separate the surfaces and the factor, $1 / 2$, takes into account the fact that two surfaces are produced. Another condition is that $\sigma=\sigma_{c}$, when $\mathrm{d} \sigma / \mathrm{dy}=0$. From this it follows that $\mathrm{y}^{*}=1 / \mathrm{a}$ and

$$
\sigma_{c}=\frac{A}{a e}
$$

where $e$ is the base of natural logarithms. Hence:

$$
\sigma_{c}=e^{-1} \sqrt{2 \mathrm{E} \gamma / \mathrm{y}_{\mathrm{o}}}=0.52 \sqrt{\mathrm{E} \gamma / \mathrm{y}_{\mathrm{o}}} .
$$

The fourth method (figure 1D) uses the we11-known Morse potential function to represent the potential energies of atoms in a crystal. This can be evaluated in terms of the thermal expansion coefficient as follows. The energy, $U$, as a function of separation distance, $y$, is written:

$$
U\left(y-y_{0}\right)=U_{0}\left[e^{-2 S\left(y-y_{0}\right)}-2 e^{-S\left(y-y_{0}\right)}\right]
$$

where $U_{0}$ is the binding energy between surfaces per average atomic area, $S$ is the reciprocal of the "range" of the interatomic forces, and $y_{0}$ is the initial separation distance. Then, the stress between two internal surfaces is:

$$
\sigma\left(y-y_{0}\right)=\frac{1}{A_{0}}\left(\frac{\partial U}{\partial y}\right)=\frac{2 S U_{0}}{A_{0}}\left[e^{-S\left(y-y_{0}\right)}-e^{-2 S\left(y-y_{0}\right)}\right] .
$$

The maximum (cohesive) stress occurs when:

$$
\frac{\partial \sigma}{\partial y}=0=\frac{2 S^{2} U_{0}}{A_{0}}\left[2 e^{-2 S\left(y-y_{0}\right)}-e^{-S\left(y-y_{0}\right)}\right] .
$$

Hence, the bracketed term must equal zero. Taking logarithms, at the maximum:

$$
\mathrm{y} *=\mathrm{y}_{\mathrm{o}}+\frac{\mathrm{ln} 2}{\mathrm{~S}} \text {. }
$$

Substituting this, to find the maximum stress:

$$
\sigma_{c}=\frac{S U_{o}}{2 A_{o}} \text {. }
$$

Next, the constants are evaluated. A change in stress, according to Hooke's Law, is given by:

$$
\mathrm{d} \sigma=\mathrm{C}_{11}^{\prime} \frac{\mathrm{dy}}{\mathrm{y}_{\mathrm{o}}}
$$


where $C_{11}^{\prime}$ is the crystal elastic stiffness in the direction of interest. Then:

$$
C_{11}^{\prime}=\frac{y_{0}}{A_{0}}\left(\frac{\partial^{2} U}{\partial y^{2}}\right)_{y=y_{0}}=\frac{2 y_{0} U_{0} S^{2}}{A_{0}}
$$

This gives one relation between $U, S$, and physical properties. Another relation is obtained by considering thermal expansion, as it relates to the first anharmonic term.

At $y=y_{0}$, the Morse potential and its first three derivatives have the follow-
values: ing values:

$$
\begin{aligned}
U(0) & =-U_{0} \\
U^{\prime}(0) & =0 \\
U^{\prime \prime}(0) & =2 S^{2} U_{0} \\
U^{\prime \prime}{ }^{\prime}(0) & =-6 S^{3} U_{0}
\end{aligned}
$$

Therefore, the Taylor expansion about the point $\mathrm{y}=\mathrm{y}_{0}$, is:

$$
U\left(y-y_{0}\right)=-U_{0}+C\left(y-y_{0}\right)^{2}-G\left(y-y_{0}\right)^{3}
$$

where:

$$
\begin{aligned}
& \mathrm{C} \neq \mathrm{U}_{\mathrm{O}} \mathrm{S}^{2} \\
& \mathrm{G} \neq \mathrm{U}_{\mathrm{O}} \mathrm{S}^{3}
\end{aligned}
$$

This represents an anharmonic oscillator whose average displacement during thermal excitation at high temperatures [Kittel, 1956; p. 152] is:

$$
\overline{\mathrm{y}}=\frac{3 \mathrm{kT}}{4} \frac{\mathrm{G}}{\mathrm{C}^{\mathrm{e}}}=\frac{3 \mathrm{kT}}{4 \mathrm{U}_{\mathrm{O}} \mathrm{S}}
$$

where $\mathrm{k}=$ Boltzman's constant, and $\mathrm{T}=$ absolute temperature. Thus, the thermal expansion coefficient normal to the plane of interest is:

$$
\alpha_{0}=\frac{\bar{y}}{y_{0} T}=\frac{3 k}{4 y_{0} U_{0} S}
$$

Using this second relation for $\mathrm{U}_{\mathrm{O}}$ and $\mathrm{S}$, we can solve for the cohesive stress:

$$
\sigma_{c}=\frac{3 k}{8 \alpha_{o} v_{o}}
$$

where $\mathrm{V}_{0}=\mathrm{y}_{\mathrm{A}}=$ average atomic volume and it is concluded that the cohesive stress should be infersely proportional to the high-temperature thermal-expansion coefficient ${ }^{3}$.

The inverse relation between cohesive stress and thermal expansion coefficient is not unexpected since empirical relations between such properties as hardness and melting point have been described in the past (see, for example, Zwikker [1954], p. 159). However, the author is not aware of previous use of the anisotropy of the easily measured thermal expansion coefficient to estimate the anisotropic cohesive stress.

All of the calculations listed above have not taken into account the Poisson contraction of a crystal as it is extended. Therefore, to obtain the cohesive stress for unaxial tension, all the $\sigma_{c}$ 's should be increased by about 10 percent.

In order to compare the various estimates of cohesive stress and to indicate the extreme upper limit that can be expected, values for the cases of diamond and NaCl are listed in table I. Method 3 is the most conservative and in best agreement with the detailed calculation of Zwicky for NaCl. Therefore, it is considered to be the best estimate. This means that the absolute upper limit of tensile strength for the foreseeable future is about $10^{12} \mathrm{~d} / \mathrm{cm}^{2}$; or about $14 \times 10^{6} \mathrm{psi}$. This is about an order of magnitude greater than the highest stress that has been observed up to now.

\footnotetext{
${ }^{3}$ High temperature means above the Debye temperature where the Dulong-Petit Law holds.
} 
Consideration of the relations between chemical constitution and strength is important for two reasons. First, because it indicates what compounds are likely to have the greatest strength; and second because it shows why careful attention to physical structure is so important for high strength.

As measures of chemical bond strengths, the elastic moduli of crystals are convenient. Numerous measurements have been made since the advent of the ultrasonic pulse-echo method, and as was discussed in the last section, the cohesive stress equals approximately 10 percent of the modulus. One relationship that is pertinent is that between cohesive stress and interatomic spacing which arises because of the fact that the primary cause of binding is electrostatic attraction. This is particularly clear in the case of ionic crystals where 90 percent of the binding energy results from attraction between the oppositely charged ions. In metals, the binding results from electrostatic attraction between the positive ions and the free electron gas. The quality of electrostatic forces that is of special interest here is their sensitive dependence on the distance between charged particles.

The electrostatic forces between atomic particles are inversely proportional to the square of the distance between particles; and, since the cross-sectional diameter of a pair of atoms is approximately equal to the distance between them in a molecule, the electrostatic stress becomes inversely porportional to the fourth power of the interatomic distance. This puts a premium on having small tightly packed atoms in a material in order to acheive high strength. Figures 2, 3, 4, 5, 6, and 7 show, for several types of binding and crystal structure, that the elastic moduli are indeed very sensitive to interatomic spacing and often inversely proportional to the inverse fourth power of the interatomic distance.

For atoms of a given size, chemical valence is also important in determining bond strengths. This is illustrated by the case of $\mathrm{MgO}$ in figure 2 whose modulus (open circle) lies well above the line for the other rock-salt type crystals when it is not corrected for the valence factor. When the measured value is divided by four to correct for the divalence it falls near the line for monovalent crystals (solid circle). The importance of valence is also shown in figure 8 which compares the variation of atomic radii with atomic number and elastic moduli. Where the variation of the radii is least, the variation of the modulus is greatest.

Of the elements, the light ones such as $\mathrm{Be}, \mathrm{B}$, and $\mathrm{C}$ are strong because of their small sizes; and the transition metals are strong because of their high effective valences. It is not surprising therefore that compounds formed between these substances are among those having the highest known elastic moduli. Certain other combinations of elements e.g., CaO, Tho, form very stable molecules, but relatively weakly bonded crystals. The compounds with highest cohesive strengths are of the interstitial type (BeO, $\mathrm{Al}_{2} \mathrm{O}_{3}, \mathrm{TiC}$, WC, $\mathrm{TiB}_{2}$, etc.), plus a few covalent compounds ( $\mathrm{SiC}, \mathrm{AlP}$, etc.).

Carbon appears to be more effective in forming strong interstitial compounds than boron or nitrogen as shown by a comparison of melting points in table 2 . This table shows that in the case of three transition metals ( $T i, V$, and $\mathrm{Ta}$ ) the carbide always has a higher melting point than the boride or nitride. Furthermore, it is known that carbon is not only effective in raising the elastic moduli of crystals, but is also effective in raising the resistance of crystals to plastic flow.

The effect of adding carbon to various metals on their elastic moduli is shown in table 3. The strength improvement is substantial in most cases, and on the average is more than a factor of two. From this it may certainly be concluded that the ultimate in strength will not be obtained using pure metals, but will require compounds. Whether simple metallic carbides are the most strongly bonded of all crystals is not known at present. There are some indications that mixed carbides, carboborides, or carbonitrides might be stronger. Carbon-carbide (diamond) is the hardest material that is known, but its ultimate strength in tension might not be as great as that of some anisotropic crystals. An extreme example is graphite which has a higher elastic modulus than diamond in the direction parallel to the graphite layers. However, some crystal that is not so weak as graphite in the direction perpendicular to its layers would seem to be more useful. Hexagonal SiC, or $\mathrm{TiB}_{2}$, might be an example.

Crystals that hold the most promise as ultra-strong materials are listed in table 4. Only those with known elastic moduli are considered, so many materials have been overlooked. The various crystals are ranked on the basis of their absolute strength ( 5 percent of $\mathrm{E}$ ), and on the basis of their anticipated strength/weight ratios (Young's modulus divided by density). From the standpoint of absolute strength, some of the 
transition metal carbides like WC and TiC appear superior to nonmetallic crystals. However, because of the small masses of their atoms, the nonmetallic crystals have better strength/weight ratios. Amongst the more or less isotropic crystals, diamond is the king from both the standpoint of absolute strength and strength/weight. As crown princes, things like cubic $\mathrm{BN}, \mathrm{TiB}_{2}, \mathrm{SiC}, \mathrm{B}_{4} \mathrm{C}$, and $\mathrm{B}$ also look very good.

The atomic properties that confer high strength are illustrated by table 4 . It was mentioned earlier that, since the binding in crystals is primarily electrostatic in character, small interatomic spacings and high valence tend to give high strength. Both of these factors are involved in the strengths of table 4. The small size and relatively high valence of carbon atoms give diamond its high strength. One whole group of high-strength crystals is formed from various combinations of the small atoms (N, B, Si, A1, P, etc.) that lie near carbon in the periodic table of elements. Another group is made up of atoms that are larger in size, but have higher valence to compensate. These are the transition metals that form carbides, nitrides, borides, etc.

The data of table 4 indicate that, without being unduly optimistic, it should be possible to make materials with strengths up to $5,000,000 \mathrm{psi}$. This is 10 times better than present steels, and from the point of view of strength/weight is more than 20 times better. Furthermore, it is important to note that strong crystals can be formed from very inexpensive raw materials. In the beginning, fabrication costs will be high, but there is no apparent reason why the cost of these materials cannot eventually be quite low.

It is likely that crystals with higher strengths than some of those listed in table 4 will be synthesized in the future. It is considerably less likely that anything with a strength greater than that of diamond will be formed. Nevertheless, since understanding of the chemical factors that provide high strength is meager, chemical studies should guide progress toward the highest possible strength.

\section{Weakening Effects of Grain Boundaries}

Since the bonding of atoms in crystals depends very strongly on interatomic spacings, and the spacings are disturbed near grain boundaries, it is expected that boundaries will have less strength than the crystals themselves. The effect is rather small in the case of metals so it is not difficult to obtain high-strength polycrystalline metals. The reason that the effect is small is that the cohesive energy in metals is insensitive to the exact arrangement of the atoms, much of it originating from long range interactions. However, in ceramic crystals, atomic arrangements are far more important because of the directionality of covalent bonding, and its short range character. Therefore, it is expected that the energies of grain boundaries in ceramics will be higher relative to free surface energies than in the case of metals. As will be shown shortly, this will cause severe weakening.

In addition to structural weakness, boundaries between anisotropic crystals are subject to severe thermal stresses. These are difficult to calculate accurately, but an estimate suggests that they can be extremely high. For example, consider two crystals bound together across a boundary that is three atoms (7A) thick. Suppose that the difference in thermal expansion coefficients between the two crystals is moderate, say $10^{-6} /{ }^{\circ} \mathrm{C}$. Then a temperature change of $100{ }^{\circ} \mathrm{C}$ will produce a displacement difference of $10^{-4} \mathrm{~cm} / \mathrm{cm}$. Suppose the grain size is such that the boundary is $10^{-2} \mathrm{~cm}$ in length. Then the maximum displacement will be $10^{-6} \mathrm{~cm}$. This could cause a displacement gradient (shear strain) across the boundary as large as 1300 percent which would be certain to cause shear fracture. Of course, elastic strains in the crystals adjacent to the boundary might relieve some of this strain, but a large fraction of it would still remain.

There are various ways for estimating the structural weakening at grain boundaries. A few of these will be considered in turn:

(a) Examination of the bubble raft photographs of Lomer and Nye (1947) suggests that about 35 percent of the atomic bonds are completely missing in a high-angle grain boundary. Thus a strength reduction of at least this amount must occur, even in metals. However, this would be an underestimate for a covalent or ionic crystal because it does not take into account the abnormal atomic positions.

(b) A formal method consists of considering the energetics of crack formation at a boundary. The geometric situation is shown in figure 9 . The applied stress, $\sigma$, produces a strain energy density in the material of $\sigma^{2} / 2 \mathrm{E}$ where $\mathrm{E}$ is the elastic modulus. Introduction of a crack of length, $2 \mathrm{~L}$ causes three changes. First, the strain energy in a volume of $\sim \pi L^{2}$ (for unit thickness) is relaxed. This amount of energy is: 
$-\pi \sigma^{3} L^{2} / 2 E$. Second, some grain boundary, of energy $-2 L \gamma g b$, is eliminated. Third, two new free surfaces, of energy $+4 \mathrm{~L} \gamma_{\mathrm{s}}$, are created. In opdex for the crack to propagate there must be a net decrease in the energy of the system. That is:

$$
\frac{\pi \sigma^{2} L^{2}}{2 \mathrm{E}} \geq\left(2 \gamma_{s}-\gamma_{g b}\right) 2 L
$$

where $\sigma$ is the stress for fracture in the presence of the grain boundary. If no boundar $b_{i s}$ present $\gamma_{g t}=0$ and the stress for fracture is $\sigma_{f}$. Thus the ratio of the fracture stresses in the two cases (eliminating the approximation made for the strain energy term) is:

$$
\frac{\sigma_{f b}}{\sigma_{f}}=\sqrt{1-\gamma_{g b} / 2 \gamma_{s}}
$$

and we see that, if the grain boundary energy equals twice the free surface energy, the strength of the boundary is zero.

For metals, eq. (4) predicts only a small change in strength at grain boundaries because the ratio $\gamma_{\mathrm{gb}} / 2 \gamma_{\mathrm{s}}$ is quite small. In the case of copper [McLean, 1957], $\gamma_{\mathrm{gb}}=$ $650 \mathrm{ergs} / \mathrm{cm}^{2}$ and $\gamma$ gb $1700 \mathrm{ergs} / \mathrm{cm}^{2}$, so $\gamma_{\mathrm{fb}} / \gamma_{\mathrm{f}}=0.9$. Unfortunately, there are no data for adequatels pure ceramics. The data of Kingery [1954] are the only ones available and they were obtained for material of ordinary purity.

In covalent substances, one expects that $\gamma_{\text {gb }}$ for high angle boundaries will be quite large because very few of the bond distances or angles will have their normal values. This is very important in this case because the cohesive forces are short range ones between individual pairs of atoms [Leman and Friedel, 1961]. Changes in the bond distances and angles are not nearly so important in metals. In a random polycrystal, there will exist some boundaries across which the misorientation angle, $\theta$ is at least $2 \pi / 2 n$ where $n$ is the maximum rotational symmetry of the crystal (for a cubic crystal $\mathrm{n}=4$ ). The shear strain corresponding to such a misorientation will be $\sim$ tan $\theta$ or $\tan \pi / 4=1 / \sqrt{2}$. Such a large strain exceeds the strain for the maximum cohesive stress, so the strain energy density will be approximately equal to the cohesive stress. Therefore, such a boundary would have zero strength.

On the basis of the argument just above, it seems quite probable that some of the grain boundaries in polycrystals of a substance such as diamond would be quite weak, even in a very pure specimen. This will be equally true of any substance whose cohesion is based on nearest neighbor interactions.

(c) A general argument can be based on the behavior of twin boundaries in nonmetallic substances. It is extremely difficult to obtain unambiguous information about the strength of grain boundaries because there is a strong tendency for impurities to segregate at them. Twin boundaries, however, can sometimes be produced mechanically at temperatures that are low enough to prevent any impurity segregation. It is observed, for example in $\mathrm{Al}_{2} \mathrm{O}_{3}$ [Stofel and Conrad, 1962], that fracture occurs preferentially at such twin boundaries. In comparison with a general grain boundary, a twin boundary has considerable symmetry and hence lower energy. Therefore, if twin boundaries in a material are weak, it is expected that general high-angle boundaries will be even weaker.

Although it is not conclusive evidence, the fact that polycrystalline nonmetallic substances commonly fracture intergranularly suggests boundary weakness as would be expected from the previous arguments. This may result from impurity effects, but it is the opinion of the present author that grain boundary weakness is an intrinsic characteristic of refractory nonmetallic substances.

\section{Dislocations in Ceramic Crystals}

Although the general geometric features and overall behavior of dislocations are independent of crystal type, certain properties are qualitatively different between metallic crystals on the one hand, and nonmetallic ones on the other. This results from the prime importance of nearest neighbor interactions in nonmetallic crystals. It is strikingly illustrated by figure 10 which compares the variation of hardness with elastic modulus for covalent and metallic crystals 「Gilman, 1960].

The upper line in figure 10 is drawn through the data for crystals with the zincblende structure which are insulators or semiconductors. The lower line correlates data for several face-centered-cubic metals. In both cases the hardness is proportional to the elastic modulus, but the proportionality constants differ by a factor of 500 . 
Hardness is a measure of the yield stress, or the ease with which dislocations move in a crystal. Thus for metals, in which dislocations move easily, the hardness value is a small fraction of the elastic modulus (about $5 \times 10^{-3}$ for the data shown). In contrast, the hardnesses of covalent crystals are a much larger fraction of the moduli (about 10 percent). Since this is close to what the cohesive shear strength is expected to be, it is concluded that dislocations have great difficulty in moving through covalent crystals. The reason for this is suggested by figure 11 .

The solid circles in figure 11 are arranged as the atoms are along the direction of glide in the diamond (or zincblende) crystal structure. The central core of a dislocation line is shown at two positions of symmetry. Only one atomic plane is shown, but the next one behind the one shown has the same geometric form; it is simply shifted by $b / 2$ with respect to the ribbon shown. Since nearest neighbor interactions determine the behavior, only the one strip needs to be considered. According to the data of table 1 , a covalent bond breaks at a strain of about 65 percent. Therefore, the energy of the dislocation core changes markedly between the two configurations, $B$ and $C$. In the configuration $B$, the equivalent of one bond is broken and at $C$, roughly two bonds are broken. As the dislocation moves along then, its energy fluctuates by an amount equal to one $\mathrm{C}-\mathrm{C}$ bond. The period of the fluctuations is $\mathrm{b} / 2$, and very little of the energy is conserved, most of it being converted directly into heat. Then, since the force that acts per atomic plane is approximately $\tau b^{2}$ (where $\tau$ is the applied shear stress), the work done per fluctuation is $\mathrm{Tb}^{3} / 4$. This must equal the bond energy of $6.22 \times 10^{12}$ ergs, so the required shear stress is $\sim 10^{12} \mathrm{~d} / \mathrm{cm}^{2}$ or about $\mathrm{C}_{44} / 10$ as suggested by fig-
ure 10 .

The idea that nearest neighbor interactions control dislocation motions in covalent crystals is given further support by recent measurements of Chaudhuri, Patel, and Rubin [1962]. They measured the velocities of individual dislocations in severai crystals (Ge, $\mathrm{Si}, \mathrm{GaSb}$, and InSb) as a function of stress and temperature. They found that the temperature dependences of the dislocation velocities in these crystals can be described by Arrhenius equations. The activation energies that they determined from these equations are shown in figure 12 to be proportional to the elastic shear stiffnesses of the crystals. Furthermore, it may be noted that the activation energies for dislocation glide are quite nearly equal to the energies of single bonds in Ge and $\mathrm{Si}$ as given by Pauling [1961]. Thus the rate determining step for dislocation glide through these crystals is the breaking of individual chemical bonds.

Again it should be emphasized that many of the properties of dislocations are quite different in nonmetals than in metals because of the short range cohesive forces. This not only makes it difficult for dislocations to move in these crystals, but also makes the physical properties of the crystals very sensitive to the presence of dislocations. There is much evidence that the electrical, magnetic, and chemical properties of nonmetallic crystals are strongly affected by dislocations. There is also some evidence that the cohesion may be strongly affected [Gilman, 1961]. Since, according to eq. (3), the cohesive stress depends directly on the thermal expansion coefficient, a simple method for studying the effect of dislocations on cohesion would be to measure the thermal expansion coefficient as a function of dislocation density. This should yield the relative reduction in cohesive stress per dislocation which is expected to be higher for nonmetals than for metals. Plastic strains are known to produce distinct, but small( 3 to 5 percent), increases in the thermal expansion coefficients of metals [Hordon, Lement, and Averbach, 1958].

\section{Strength and Structure}

Since a material can be no stronger than its weakest part, and it has been emphasized above that interatomic distances are of prime importance in determining strength, it follows that the internal structure of a strong material should consist of atoms that are packed together as closely as possible. This demands that the material be crystalline because the atoms in crystals are in a state of maximum packing density. Also, it rules out glasses and high polymers because they are not sufficiently dense.

It is quite clear that an ultra-strong material cannot contain gross defects such as voids, cracks, or foreign inclusions. As was discussed in a previous section, it probably cannot contain grain boundaries either. These are not severe defects in metals, but the available evidence indicates that they severely weaken many nonmetals. Defects that are still less severe than grain boundaries are individual dislocations and atomic vacancies. If these defects are present in a crystal in high concentration, or if they can move around readily, there is no doubt that they cause a substantial loss of strength. However, if they are few in number and not mobile, they can probably be tolerated without a severe loss of strength. 
A structure that has often been suggested and discussed in recent years is that of a composite consisting of a bundle of very fine whisker-like crystals embedded in a polycrystalline or glassy matrix. There have been many observations that crystals in whisker form have very high strength, and the hope is that this strength can be exploited in a composite. The author has no confidence in this approach. It might be useful in very special situations, but it has serious difficulties as an approach to ultra-strength. The first difficulty, and perhaps the easiest to overcome, is the problem of obtaining a high packing-fraction for small fibers through an economic process that does not spoil the high strength of the fibers. Another serious difficulty is that if the matrix is softer than the reinforcement fibers, stress concentrations will inevitably arise when the material is stressed and these are likely to lead to fracture. If the matrix has a hardness similar to or greater than the fibers, then there is little point in putting fibers into the matrix. A third difficulty is that of forming strong interfaces between the matrix and the fibers. The interfaces can be expected to behave no better than grain boundaries which at best do not seem to have great strength.

It is concluded that ultra-strong materials will take the form of monocrystals. The monocrystals must be structurally sound and have very high elastic stiffnesses. Defects no larger than atomic dimensions (dislocations, impurities, vacancies) may be present in them, but nothing larger.

\section{Feasible Strengths for Monocrystals}

It might be thought that some elaborate method of preparation would be required to produce an ultra-strong material. Actually, quite simple methods suffice. The solid itself must be of high structural quality, and then it is simply necessary to put very smooth surfaces on it. Surface notches would severely lower the strength so they must be eliminated by various methods described below.

Crystal "whiskers" grow with very good surfaces so no further preparation is necessary for them to be very strong (Brenner, 1958). However, it is difficult to grow sufficiently large whiskers for most practical uses.

Another method that yields smooth surfaces is that of drawing out a slender rod. Hillig [1961] has shown that if commercial fused silica rods are drawn down from $13 \mathrm{~mm}$ diam to 0.5 to $3.0 \mathrm{~mm}$ diam in an oxyhydrogen flame, they exhibit strengths up to $1,900,000$ psi at $78^{\circ} \mathrm{K}$. Figure 13 shows one of his rods bent to a maximum elastic strain of about 10 percent. This is the largest elastic strain that has ever been observed in such a large specimen.

Perhaps the most versatile method for preparing smooth surfaces is chemical polishing. This allows a desired shape to be cut out first and then polished to give it high strength. Dash [1958] has shown that strong beams of silicon can be produced in this way, and Gilman [1961] has done the same for rings. One of the latter, prepared by abrasive cutting from a commercial silicon crystal is shown in figure 14 . It withstood a maximum stress of about 500,000 psi before it broke.

Since the elementary structural members described above have already been made from strong brittle materials, there is no reason to doubt the general feasibility of using strong monocrystals in mechanical structures. The only real questions that remain with respect to practicality concern reliability, and the cost of producing monocrystals in large sizes and quantities.

The maximum strengths that have already been observed for various substances are 1isted in table 5. These data are quite encouraging to the idea that strengths up to $\mathrm{E} / 10$ can be attained. When carbides (and diamond) become available in large enough sizes to make machine elements, crystals will become increasingly attractive as engineering materials. The vision of diamonds or other crystals being used in extremely strong tensile structures is an exciting one. If it becomes a reality in the relatively near future, the interest of many men in understanding the mechanical behavior of crystals will have been vindicated in the most concrete of terms.

\section{The Ductility Problem}

There is no doubt that it will be difficult to design machines that use ultrastrong materials for structural parts. It cannot be expected that these materials will have ductility because ultra-high strength and ductility are incompatible properties. In order to compete successfully, an ultra-strong material must have significantly greater strength than steel. It would be ideal if this could be achieved without sacrificing ductility, but this cannot be done and still compete with steel. Steel 
is a material with remarkable strength. Not only is its elastic modulus relatively high, but also men have learned how to work it and heat treat it very skillfully in order to optimize its strength.

In order to make something that has substantially more strength than steel it becomes necessary to take considerable pains. A chief difficulty is that no material has an elastic modulus that is an order of magnitude greater than that of steel. Ductile behavior requires that a material be able to resist crack propagation by flowing plastically so as to blunt the tips of any incipient cracks. But this means that the material must flow plastically at stresses at least one (and more realistically two) orders of magnitude lower than its cohesive stress. This would wipe out any elastic modulus advantage that a material had compared to steel.

It might be argued that one should be able to match the combination of ductility and strength that is possessed by steel, relative to its elastic modulus. However, such a material would necessarily be metallic and tungsten is the only one that is reasonable in cost and significantly higher in modulus (by a factor of two). But unlike iron, tungsten is not allotropic so the microstructures that are present in steel could not be produced in it. Even if they could the result would give only a factor of two increase in the strength and, because of the high density of tungsten, the strength-weight ratio would decrease.

It is concluded that ultra-high strength can only be achieved at the sacrifice of ductility. This means no ductility at all because the crystals that we have discussed are frankly and unequivocally brittle.

When a brittle material is highly stressed it contains an enormous energy density. If it fractures, this stored energy is released in an uncontrolled fashion that pulverizes the material. For example, the author has seen a bar of silicon all but disappear in a cloud of dust when it fractured at a stress level of $750,000 \mathrm{psi}$. In a ductile material, stored strain energy can be converted into heat through plastic flow, but in a brittle material it can only be converted into surface and kinetic energy of the broken pieces; hence shattering occurs.

It is understandable that the catastrophic way in which brittle materials fracture should be disquieting to the emotions of an engineer. Nevertheless, since the use of such materials will make new engineering ventures possible, and will markedly improve old machines, it will be worthwhile to face the problem squarely. Gradually one can learn how to design structures that will perform reliably in spite of brittleness.

The virtues of a ductile material are chiefly two in number. One is that such a material can absorb impact loadings, and the other is that surface scratches on ductile materials cause negligible lowering of the load carrying capacity. Lack of plastic flow in a brittle material makes it vulnerable to undamped impact loads, and especially to surface scratches. Impact loads can be absorbed to some extent by brittle materials as increased elastic strain-energy. However, since elastic waves are not appreciably damped when plastic flow is absent, the danger is always present that a wave will become so intensified during a series of reflections that it will cause fracture. Thus sharp impulsive loads are to be avoided, and provisions for damping reflections at free surfaces are important.

A helpful circumstance in the problem of surface scratches is the fact that the stronger a material gets, the more difficult it becomes to scratch it, and the fewer is the number of materials that are able to scratch it. Surface scratches and other damage that is initially present on a surface can be removed by some form of chemical polishing (mechanical polishing is not suitable because it invariably leaves a damaged surface layer). Once a scratch-free surface has been prepared, it should be possible to protect it against subsequent damage.

There are at least two approaches to surface protection. One is to put a very hard abrasion-resistant-coating on a softer surface in order to prevent scratching. The other is to put a coating of a relatively soft, ductile metal on the surface in order to spread out the stresses produced by an abrading particle. It seems likely that the second of these two approaches is the better one. A hard surface layer might prevent scratching, but it would allow local compressive stresses to be transmitted almost unabated to the load bearing material. In combination with tensile stresses due to the main load, such compressive stresses would tend to produce high local shear stresses that might result in failure. 
In general terms, the primary function of strength is containment. The stress field within a strong solid is used to contain some other kind of field of force. The field being contained might be pressure, a magnetic field, a gravitational field, or an electric field. The stress levels that are required to contain such fields can be calculated for specific devices.

It would be pleasant to be able to use stronger materials in almost any engineering structure. However, cost dictates the use of steel in many applications, and reliability is of prime concern in almost all of the remaining ones. For example, one can hardly expect to use brittle materials for highly stressed aircraft parts at the present time, because not enough can be done to ensure reliable performance. Very strong, but brittle, materials are feasible for use in less vital machinery, however. In some cases the improvement in performance would be trivial, but in many cases a large forward stride could be made.

\section{a. Pressure Vessels}

An example of a machine whose performance is critically dependent on the strength of materials is a high-pressure press. At present, materials such as sintered tungsten carbide that are used in such presses are many times stronger in compression than tension. Therefore, presses are designed to make the ratio of compressive stresses to tensile stresses as large as possible, and this severely limits the size of the highpressure chamber that can be used. If materials with the same or greater strength in tension were available, the chamber size would be unlimited. This could greatly improve present methods of high-pressure chemical synthesis, and it would become possible to measure new physical properties at superpressures.

Present pressure vessels are limited to pressures less than about 500,000 psi so the existence of materials with strengths up to 5,000,000 psi could open up a new realm for high-pressure research.

\section{b. Centrifuges}

Another machine that is limited by strength is the ultra-centrifuge. One of the best of present ultra-centrifuges produces a force 1,000,000 times gravity in a few cubic centimeters of material. If a material with a strength of $7 \times 10^{6}$ psi were available, this could be increased to 500,000,000 times gravity, and thereby open a whole new realm to experimentation on the effects of high gravitational fields.

This is a case where performance is absolutely limited by strength. Many machine parts can be given adequate strength for a given application simply by increasing their dimensions. This is not the case for a centrifuge, however, because the maximum speed depends only on the fracture stress of the rotor material and not upon its size.

The calculations of Chree [1895] show that the most favorable shape for high-speed rotations is an elongated ellipsoidal disk rotating about its shortest axis. The maximum angular velocity for such a disk is:

$$
\omega_{\max }=\frac{2}{a} \sqrt{\sigma_{m} / 0}
$$

where $a$ is the maximum radius, $\rho$ is the density, and $\sigma_{m}$ is the maximum strength of the material. The corresponding angular acceleration is:

$$
w^{2} a=\frac{4}{a}\left(\frac{m}{2}\right)
$$

Taking values for a diamond rotor of: $\sigma_{\mathrm{m}}=10^{12} \mathrm{~d} / \mathrm{cm}^{2}, \rho=3.5 \mathrm{~g} / \mathrm{cm}^{3}$, and letting $a=1 \mathrm{~cm}$, the angular acceleration is about $10^{12} \mathrm{~cm} / \mathrm{sec}^{2}$ or about $10^{9}$ times the acceleration of gravity.

According to the general theory of relativity, centrifugal accelerations are equivalent to gravitational accelerations in their effects on physical properties. Thus the above acceleration might be used to study the effect of gravity on properties. For example, the frequency of some process occurring at the outer edge of a spinning disk might be studied. The shift in frequency of such a process would be:

$$
\frac{\Delta \nu}{\nu_{0}}=\varphi / c^{2}
$$


where $\varphi$ is the gravitational potential equal to $-\omega^{2} \mathrm{a}^{2} / 2$ and $c$ is the velocity of light. For the numerical values given in the paragraph above and $c=3 \times 10^{10} \mathrm{~cm} / \mathrm{sec}$;

$$
\frac{\Delta \nu}{\nu_{0}}=\frac{2 \sigma_{m}}{n_{c}^{2}}=\frac{2 \times 10^{12}}{3.5 \times 9 \times 10^{20}}=6.3 \times 10^{-10} .
$$

This is a very small frequency shift, but is accessible to measurement by modern methods.

Another application of high speed rotation is for producing high pressures. The pressure at any radial distance from the center of rotation is $-\rho \varphi$. Since $\varphi_{\max }=$ $20 \mathrm{~m} / \mathrm{p}$, the maximum possible pressure is $2 \sigma_{\mathrm{m}}$, or about $14,000,000 \mathrm{psi}$.

\section{c. Gyroscopes}

The restoring force on a perturbed gyroscope is proportional to its rotational speed. Thus, it should be possible to reduce the size or improve the performance of gyroscopes through the use of high-strength materials. The improvement could be at least a factor of ten, and even better on a strength-weight basis.

\section{d. Energy Storage in Flywheels}

Storage of energy could be done quite efficiently by means of flywheels or springs made of high-strength materials. Table 6 compares the volume energy densities that can be stored in various nonnuclear ways. It may be seen from these data that a highstrength flywheel has higher volume efficiency than almost any other method. Also the energy in a flywheel is readily available because it can be made part of an electrical generator, or perhaps operated directly as a homopolar generator so the energy can be extracted directly.

\section{e. Cutting Tools}

Since cutting tools often fail because of overloading in tension, such things as: lathe bits, drawing dies, and extrusion dies represent another large group of devices that could be strikingly improved by materials with ultra-strength and high hardness.

\section{f. Ultra-High-Field Magnets}

The pressure that a magnetic field exerts on the boundary of a region that contains it is $\mathrm{B}^{2} / 8 \pi\left(\mathrm{d} / \mathrm{cm}^{2}\right)$ where the magnetic flux, $\mathrm{B}$ is given in gauss. This is a primary limitation on the maximum field that can be produced, because the coil that generates the field must have enough strength to withstand this pressure as a bearing load. It limits the fields in long-lived pulsed-magnets to about 200,000 gauss [Kuskowski, Novey, and Warshaw, 1961] where beryllium-copper is used for the coil. It limits the field in short-lived magnets to 1 to $3,000,000$ gauss [Furth, 1960].

If a high-strength,high-conductivity crystal were available with a strength of $5,000,000$ psi it should be able to support continuous magnetic fields up to $3,000,000$ gauss, and pulsed fields about twice as large; say 6,000,000 gauss ${ }^{4}$. Crystals of the transition metal carbides look promising for this application because of their metallic electrical conductivity.

\section{g. Electrostatic Fields}

Analogous with the case of a magnetic field, the stress required to contain an electrostatic field is $\mathrm{E}^{2} / 8 \pi$ where $\mathrm{E}$ is the electric field in electrostatic volts/cm. Thus an insulator such as diamond, with a strength of $8.5 \times 10^{6}$ psi could, in principle, contain an electric field of $3.9 \times 10^{6} \mathrm{esu} \mathrm{V} / \mathrm{cm}$ or about $10^{9} \mathrm{C} / \mathrm{cm}$. This is far in excess of anything that has been observed as yet, and should allow the production of some interesting physical effects.

Very high electric fields, such as mentioned above, should allow the construction of very efficient electro-mechanical transducers because forces of several million pounds per square inch could be exerted by vacuum capacitors.

\footnotetext{
"If the coil were precompressed, this might be increased to 10,000,000 gauss.
} 
S. S. Brenner, Growth and Perfection of Crystals, p. 160, John Wiley \& Sons (New York, 1958).

A. R. Chaudhuri, J. R. Patel, and L. G. Rubin, Velocities and densities of dislocations in germanium and other semiconductor crystals, J. App1. Phys. (to be published).

C. Chree, Stresses and Strains in an isotropic elastic solid ellipsoid in equilibrium under body forces derivable from a potential of the second degree, Proc. Roy. Soc. London 58, $39(1895)$.

H. P. Furth, High magnetic field research, Science 132, 287 (1960).

J. J. Gilman and W. G. Johnston, Dislocations in lithium fluoride crystals, Solid State Phys. 13, 147 (1962).

J. J. Gilman, Reduction of cohesion in ionic crystals by dislocations, Appl. Phys. 32, 738 (1961).

J. J. Gilman, U1trahigh strength materials of the future, Mech. Eng. (Sept. 1961).

W. B. Hillig, Strength of bulk fused quartz, J. App1. Phys. 32, 741 (1961)

M. J. Hordon, B. S. Lement, and B. L. Averbach, Acta Met. 6, 446 (1958).
C. Kitte1, Introduction to Solid State Physics, p. I52, John Wiley \& Sons (New York, 1956).

W. C. Kingery, J. Am. Ceram. Soc. $37,42(1954)$.

R. L. Kuskowski, T. B. Novey, and S. D. Warshaw, Design and construction of a system of pulsed magnets, Rev. Sci. Instr. 32 , $674(1961)$.

F. Leman, and J. Friede1, on the description of covalent bonds in diamond lattice structures by a simplified tight-binding approximation, J. App1. Phys. Supp. 33 281 (1962).

W. M. Lomer and J. F. Nye, Proc. Roy. Soc. London A190, 474 (1947).

D. McLean, Grain Boundaries in Metals, p. 76, Oxford Press (London, 1957).

L. Pauling, The Nature of the Chemical Bond, CornelI Univ. Press (Ithaca, New York, 1960).

E. Stofel and H. Conrad, Fracture modes in sapphire crystals, J. Metals 14, 87 (1962).

C. Zwikker, Physical Properties of Solid Materials, Interscience Publishers, Inc. (New York, 1954).

TABLE 1. Cohesive stresses estimated in various ways

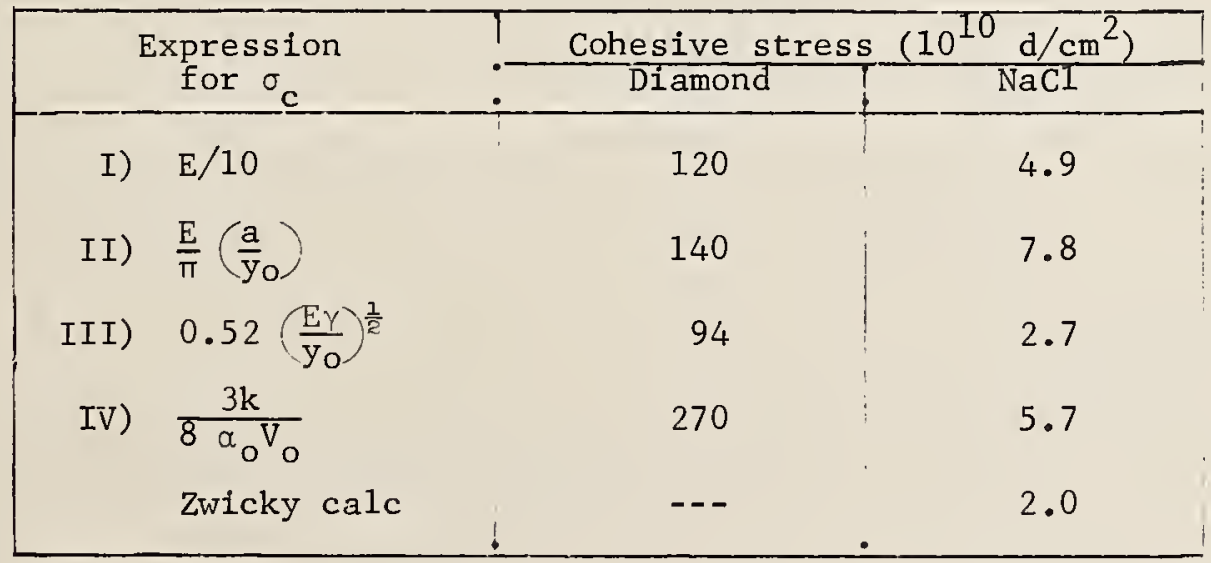

References for Table 1:

$\gamma_{111}($ diamond $)=5650$ ergs $/ \mathrm{cm}^{2}$ - W. D. Harkins, J. Chem。 Phys. 10, 269 (1942).

$\alpha($ diamond $)=3.4 \times 10^{-6} /{ }^{\circ} \mathrm{K}-\mathrm{J}$. Thewlis and A. R. Davey, Phil. Mag. 1 , 409 (1956).

$E_{111}($ diamond $)=1.2 \times 10^{13} \mathrm{~d} / \mathrm{cm}^{2} ; d_{0}=3.57 \mathrm{~A} ; \mathrm{a}=0.77 \mathrm{~A}$. 
$\mathrm{E}_{100}(\mathrm{NaCl})=4.9 \times 10^{11} \mathrm{~d} / \mathrm{cm}^{2} ; \mathrm{y}_{100}=2.81 \mathrm{~A} ; \mathrm{d}_{0}=5.63 \mathrm{~A}$.

$\alpha(\mathrm{NaCl})=41 \times 10^{-6} /{ }^{\circ} \mathrm{K}-\mathrm{A}$. Eucken, and W. Dannoh1, Z. Elektrochem. 40, 814, 1934).

$Y_{100}(\mathrm{NaCl})=155 \mathrm{ergs} / \mathrm{cm}^{2}-\mathrm{R}$. Shuttleworth, Proc. Phys. Soc。㐌2, 167 (1949).

$\sigma_{c}$ (calc. Ionic theory) - Zwicky, Physik. Z。 24, 131 (1923).

TABLE 2. Comparison of the melting points $\left({ }^{\circ} \mathrm{C}\right)$ of borides, carbides, and nitrides of some metals

\begin{tabular}{|c|c|c|c|}
\hline Metal & B & C & N \\
\hline Ti & $2020 *$ & 3250 & 2950 \\
V & 2250 & 2830 & 2030 \\
Ta & 2420 & 3875 & 3100 \\
\hline
\end{tabular}

*Decomposes

TABLE 3. Effect of carbide formation on the bond strength (elastic modulus) of various metals

\begin{tabular}{|l|c|c|}
\hline & Elastic modulus (mpsi) ${ }^{*}$ \\
\hline W & Metal & 100 \\
B & 60 & 65 \\
Ta & 50 & 43 \\
V & 27 & 40 \\
No & 24 & 50 \\
Si & 23 & 70 \\
Ti & 23 & 45 \\
Zr & 16 & 50 \\
\hline
\end{tabular}

*Million psi

IReferences for Table 3:

W. Koster, Z. Meta11k. 39,1 (1949).

R。Kieffer and P. Schwarzkopf, Hartstoffe und Hartmetalle, Springer-Verlag (Vienna 1953). 
TABLE 4. Possible strengths of various crystals

\begin{tabular}{|c|c|c|c|c|}
\hline Crysta 1 & $\begin{array}{l}\text { Young s } \\
\text { modulus } \\
\text { (mpsi)* }\end{array}$ & $\begin{array}{r}\text { Density } \\
(\mathrm{s.g.}) \\
\end{array}$ & $\begin{array}{l}\text { Maximum } \\
\text { strength } \\
(5 \% \text { strain-mpsi) } \\
\end{array}$ & $\begin{array}{l}\text { Strength: density } \\
\text { ratio } \\
\text { (arbitrary units) }\end{array}$ \\
\hline Diamond & 170 & 3.5 & 8.5 & 48 \\
\hline WC & 104 & 15.8 & 5.2 & 7 \\
\hline $\mathrm{TiB}_{a}$ & 94 & 4.5 & 4.7 & 21 \\
\hline $\mathrm{Al}_{2} \mathrm{O}_{3}$ & 76 & 4.0 & 3.8 & 19 \\
\hline $\mathrm{TiC}$ & 72 & 4.9 & 3.6 & 15 \\
\hline SiC & 71 & 3.2 & 3.5 & 22 \\
\hline $\mathrm{B}_{4} \mathrm{C}$ & 66 & 2.5 & 3.3 & 26 \\
\hline $\mathrm{ZrB}_{2}$ & 64 & 5.6 & 3.2 & 11 \\
\hline $\mathrm{W}_{2} \mathrm{C}$ & 62 & 17.3 & 3.1 & 4 \\
\hline W & 60 & 19.3 & 3.0 & 3 \\
\hline $\mathrm{MoSi}_{2}$ & 55 & 6.0 & 2.8 & 9 \\
\hline Mo & 54 & 10.2 & 2.7 & 5 \\
\hline B & 51 & 2.3 & 2.6 & 22 \\
\hline $\mathrm{BeO}$ & 51 & 3.0 & 2.6 & 17 \\
\hline $\mathrm{FeS}_{2}$ & 50 & 5.0 & 2.5 & 10 \\
\hline $\mathrm{ZrC}$ & 50 & 6.8 & 2.5 & 7 \\
\hline $\mathrm{NbC}$ & 50 & 7.8 & 2.5 & 6 \\
\hline $\mathrm{Be}_{2} \mathrm{C}$ & 46 & 2.4 & 2.3 & 19 \\
\hline $\mathrm{Be}$ & 45 & 1.8 & 2.2 & 25 \\
\hline MgO & 35 & 3.5 & 1.8 & 10 \\
\hline Si & 23 & 2.3 & 1.7 & 10 \\
\hline Steel & 28 & 7.8 & 1.7 & 4 \\
\hline
\end{tabular}

*Million psi 
TABLE 5. Highest strengths attained by various forms of solids

\begin{tabular}{|c|c|c|c|c|}
\hline Material & $\begin{array}{c}\sigma_{f}=\text { maximum } \\
\text { observed strength } \\
\text { (mpsi) }\end{array}$ & $E$ (mpsi) & $E / \sigma_{f}$ & Reference \\
\hline Music wire & 0.4 & 20 & 72 & NBS (1943) \\
\hline Silica fibers & 3.5 & 14 & 4 & Anderegg (1939) \\
\hline Silica rods & 1.9 & 14 & 7 & Hillig (1961) \\
\hline Iron whisker & 1.9 & 43 & 23 & Brenner (1958) \\
\hline $\mathrm{Al}_{2} \mathrm{O}_{3}$ whisker & 2.2 & 72 & 33 & Brenner (1958) \\
\hline NaC1 whisker & 0.16 & 6.3 & 40 & Gyula1 (1954) \\
\hline BeO whisker & 2.8 & 49 & 17 & Ryshkewitch (1962) \\
\hline Silicon whisker & 0.94 & 24 & 26 & Evans (1958) \\
\hline Silicon (bulk) & 0.75 & 24 & 32 & Pearson (1957) \\
\hline TiC (bulk) & 0.80 & 70 & 87 & Williams (1961) \\
\hline Boron & 0.35 & 51 & 145 & Talley (1959) \\
\hline Mica & 0.43 & - & - & Orowan (1933). \\
\hline Austformed steel & 0.45 & 29 & 64 & Shine (1959) \\
\hline
\end{tabular}

References for Table 5:

F. O. Anderegg, Ind. Eng. Chem. 31, 290 (1939)。

S. S. Brenner, Growth and Perfection of Crystals, p. 157, John Wiley \& Sons (New York, 1958).

C. C. Evans, reported by J. E. Gordon, in Growth and Perfection of Crysta1s, p. 219.

Z. Gyulai, Z. Physik. 1ㅗㅛ, 317 (1954).

W. B. Hillig, J. App1. Phys。 32, 741 (1961).

NBS Circ. 447, U. S. Govt. Print. Off. (1943).

E. Orowan, Z. Physik. 12,235 (1933).

G. L. Pearson, W. T. Read, and W. L. Feldman, Acta Met. 5, 181 (1957).

E. Ryshkewitch, Science and Technology (Feb. 1962).

J. C. Shine, V. F. Zackay, and D. J. Schmatz, ASM Preprint No. 163 (1959).

C. P. Ta1ley, J. App1. Phys. $\underline{30}, 1144$ (1959). 
TABLE 6. Comparison of modes of energy storage

\begin{tabular}{|c|c|}
\hline Mode & $\begin{array}{c}\text { Density of energy } \\
\text { storage } \\
\left(\mathrm{ft}-1 \mathrm{~b} / \mathrm{ft}^{3}\right) \\
\end{array}$ \\
\hline Gasoline fuel & $682 \times 10^{6 *}$ \\
\hline Solid propellant & 105 \\
\hline Gasoline engine & 99 \\
\hline $\mathrm{Ag}-\mathrm{Zn}$ cel1 & 15 \\
\hline Pb-acid cell & 5 \\
\hline Compressed gas & 2.5 \\
\hline Capacitor & 0.2 \\
\hline Steel flywheel (0.5 mpsi)** & 45 \\
\hline Silicon flywheel (0.75 mpsi) & 68 \\
\hline Quartz flywheel ( $1.0 \mathrm{mpsi}$ ) & 90 \\
\hline Silicon carbide flywheel ( 4.0 mpsi) & 360 \\
\hline Diamond flywhee1 ( $7.0 \mathrm{mpsi})$ & 650 \\
\hline
\end{tabular}

*First group of data from: Kline, Marco, and Starkey, Trans. ASME, 80, 909 (1958).

$* *$ Fracture strength in millions of pounds per in ${ }^{2}$.
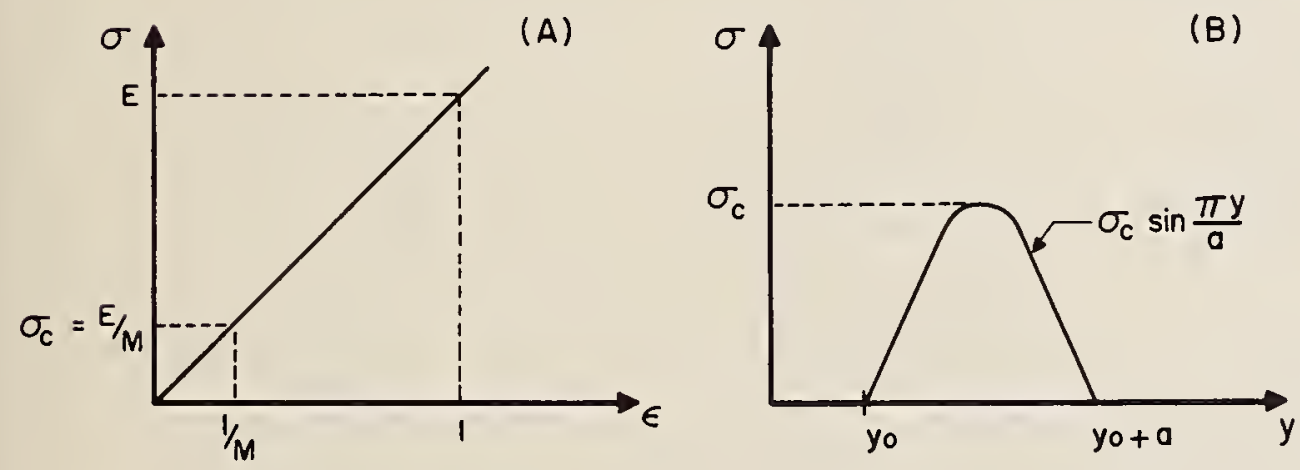

FIGURE 1. Schernatic diagrams for finding cohesive stresses in terms of various measurable physical properties.
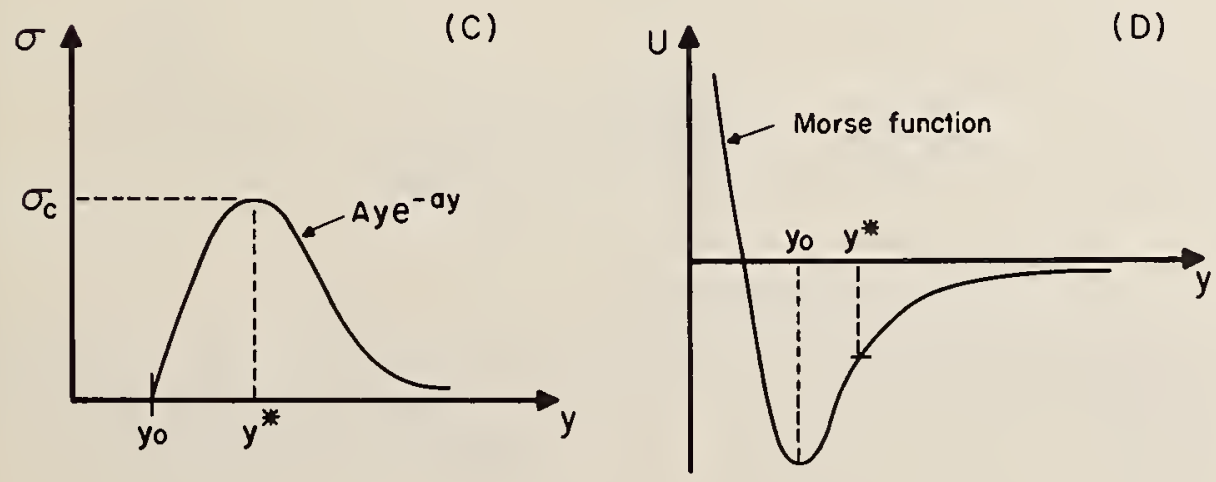

(A) Elastic modulus. (B) Elastic nodulus plus interatomic spacing.

(c) Elastic modulus, spacing, and surface energy.

(D) ilastic modulus, spacing, and therral expansion coefficient. 


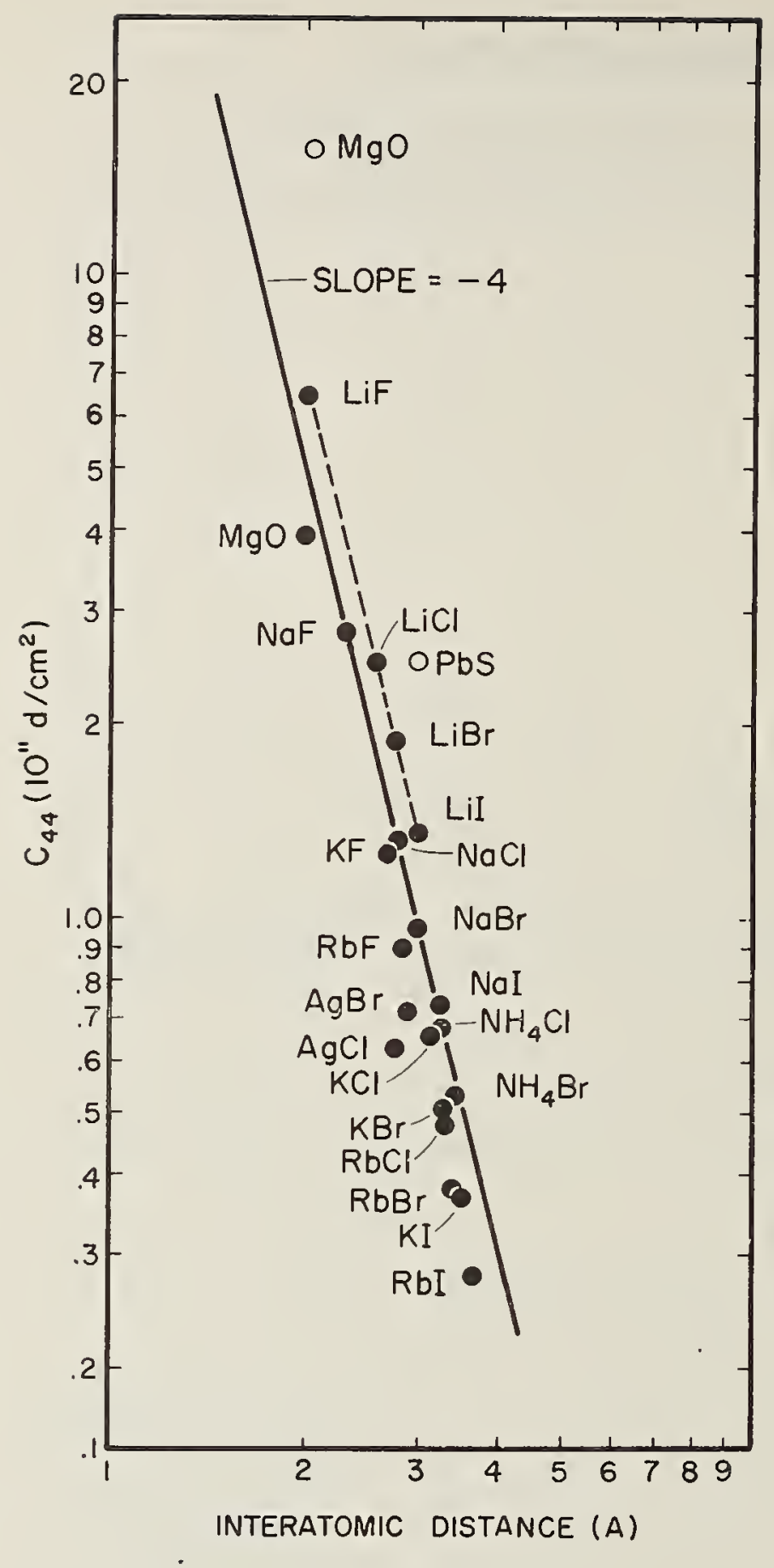

FIGURE 2. Ionic crystals with rocksalt structure.

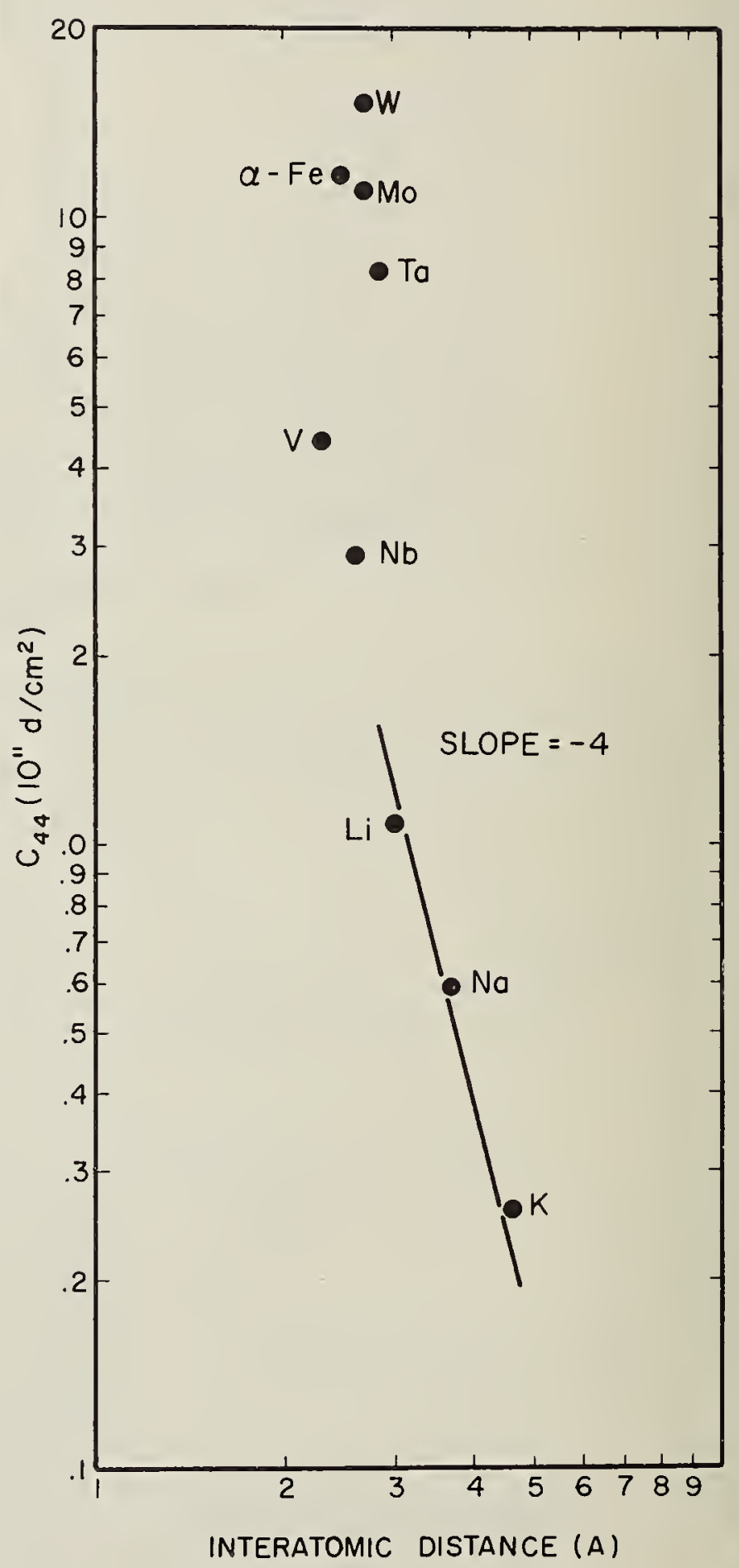




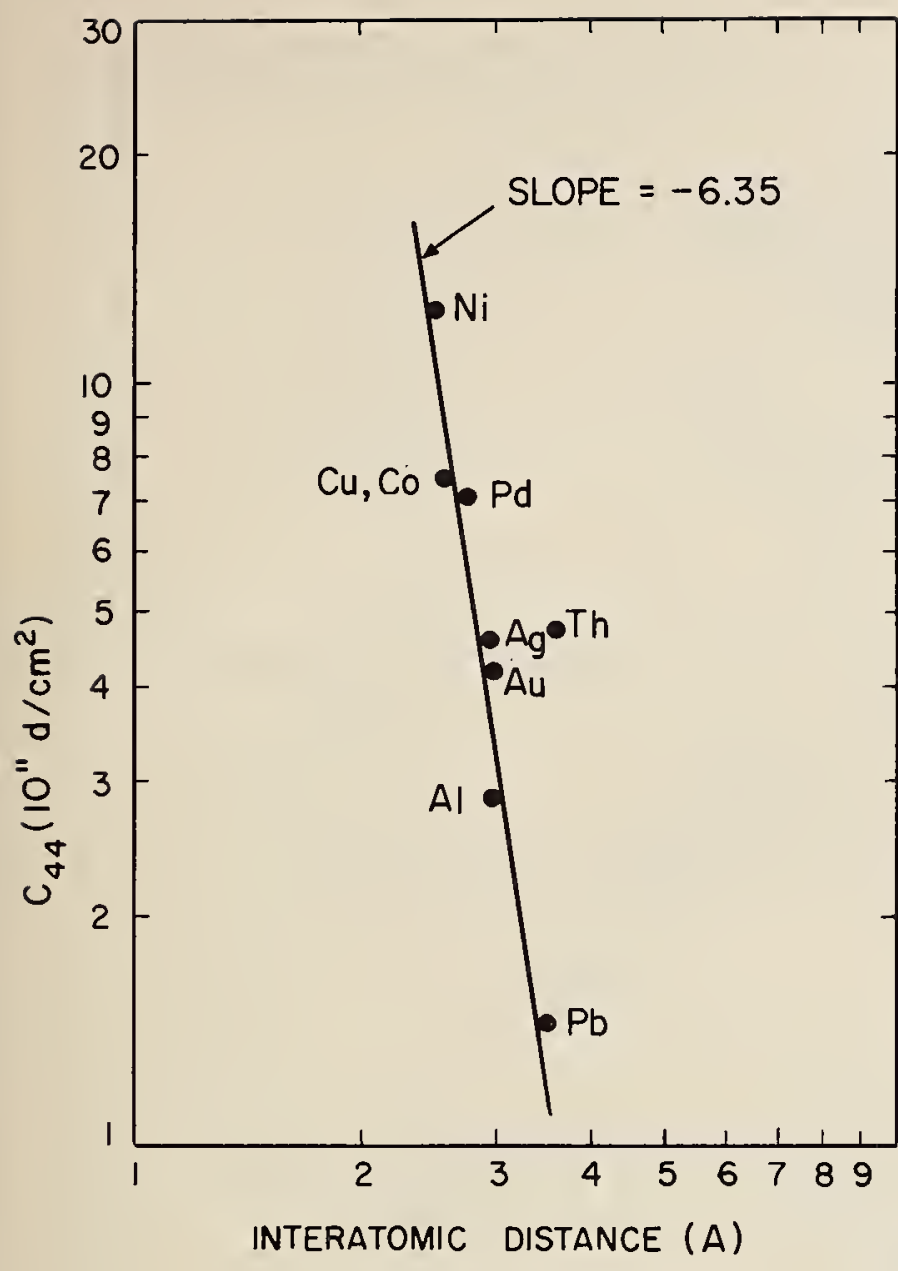

FIGURE 4. Face-centered-cubic
metals.

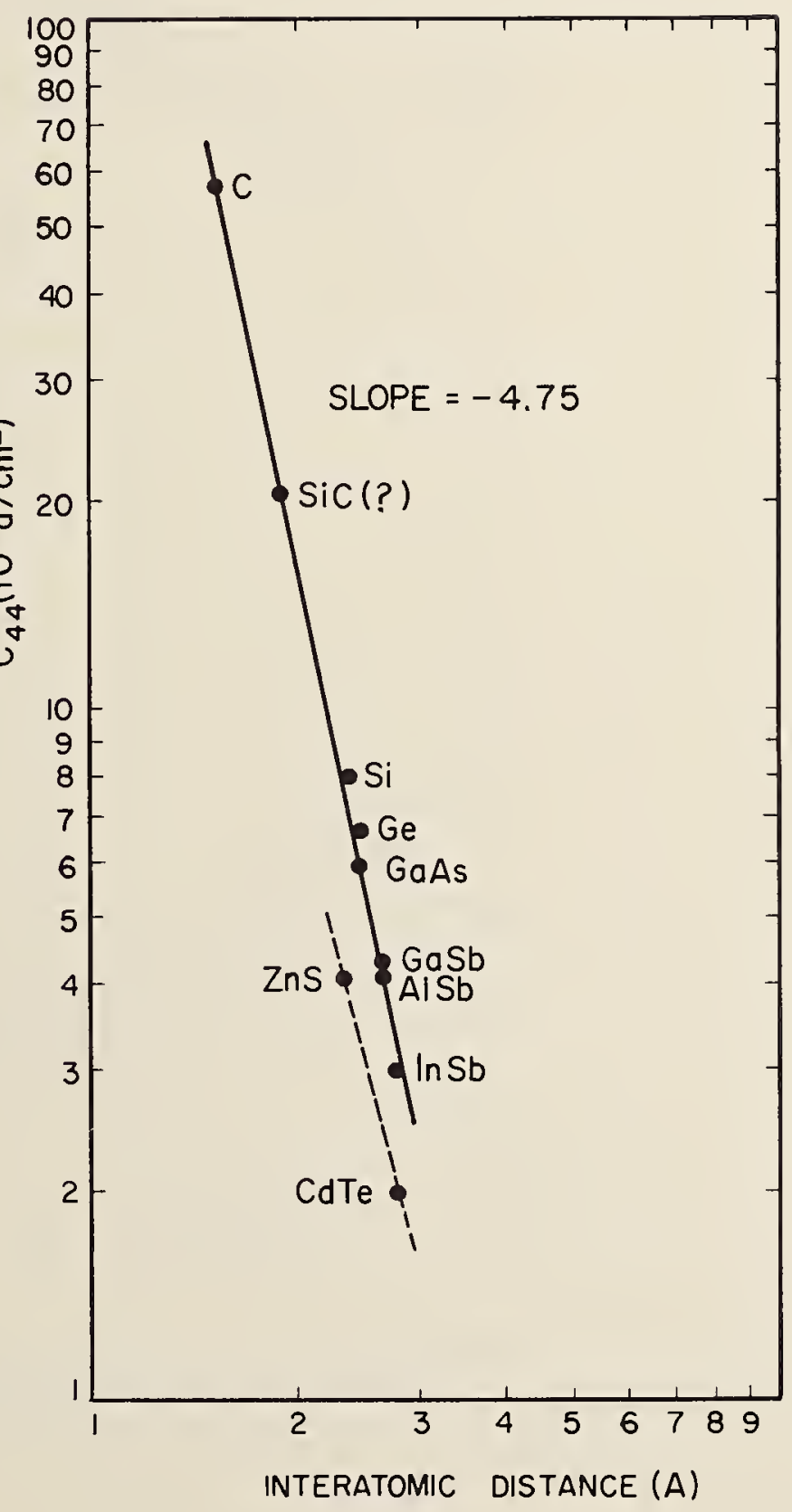




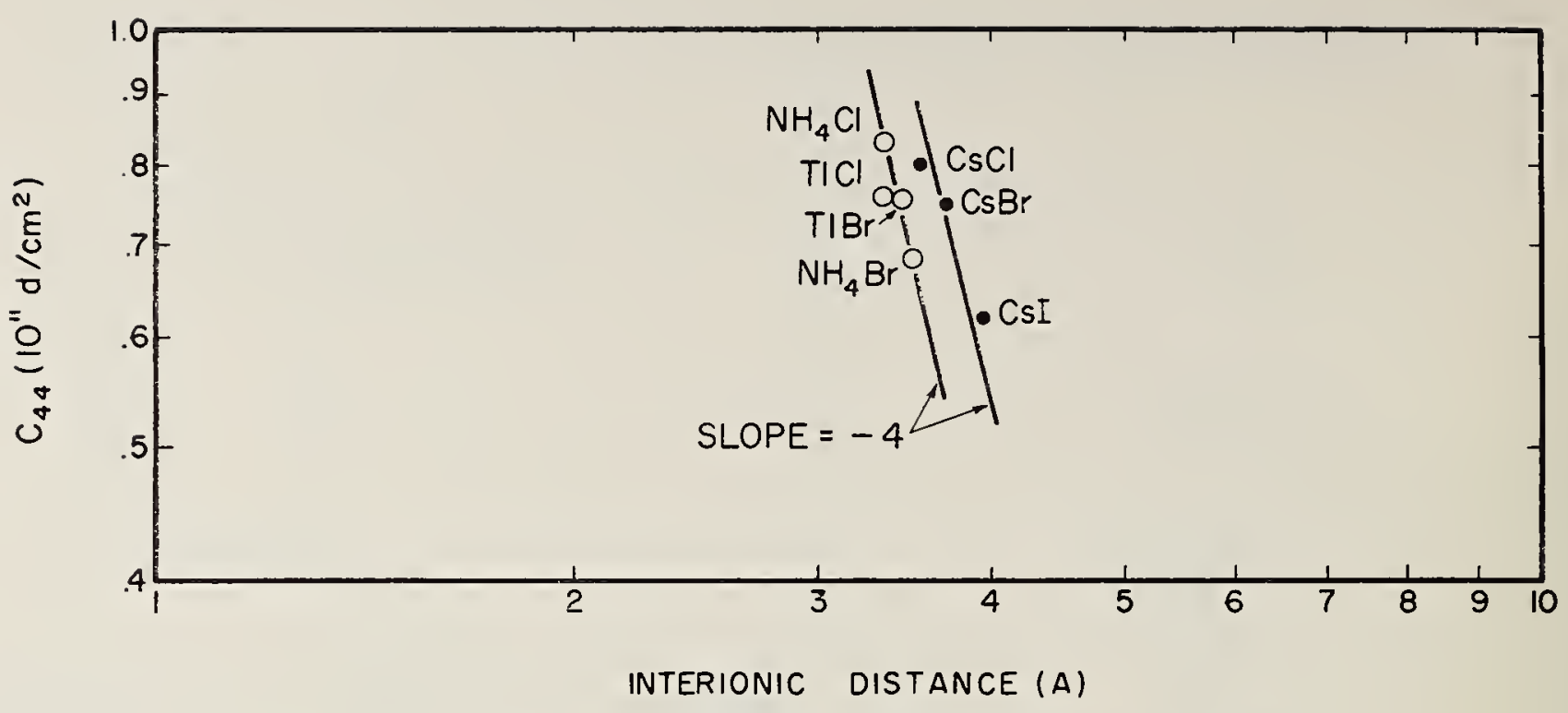

FIGURE 6. Ionic crystals with cesium chloride structure.

FIGURE 7. Hexagonal metals

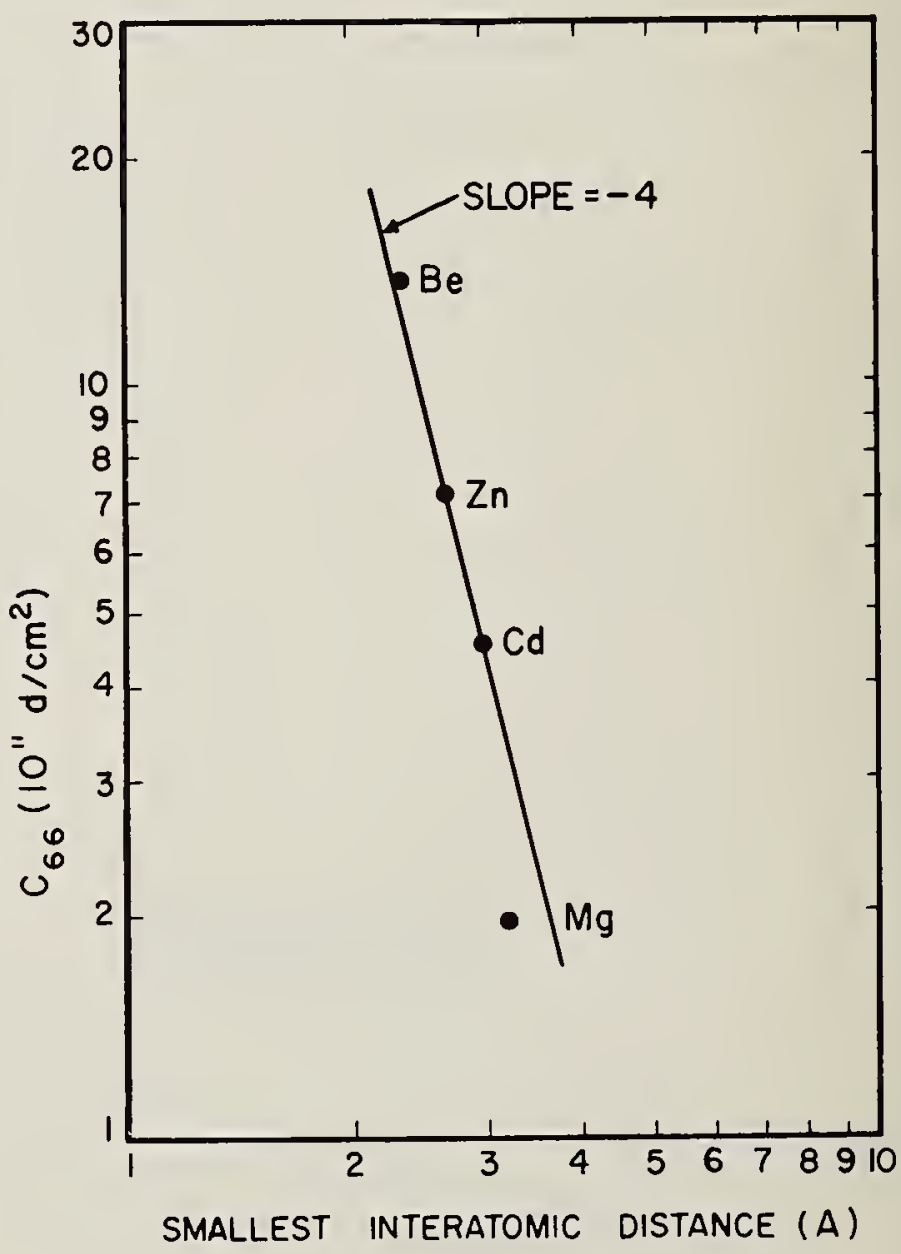



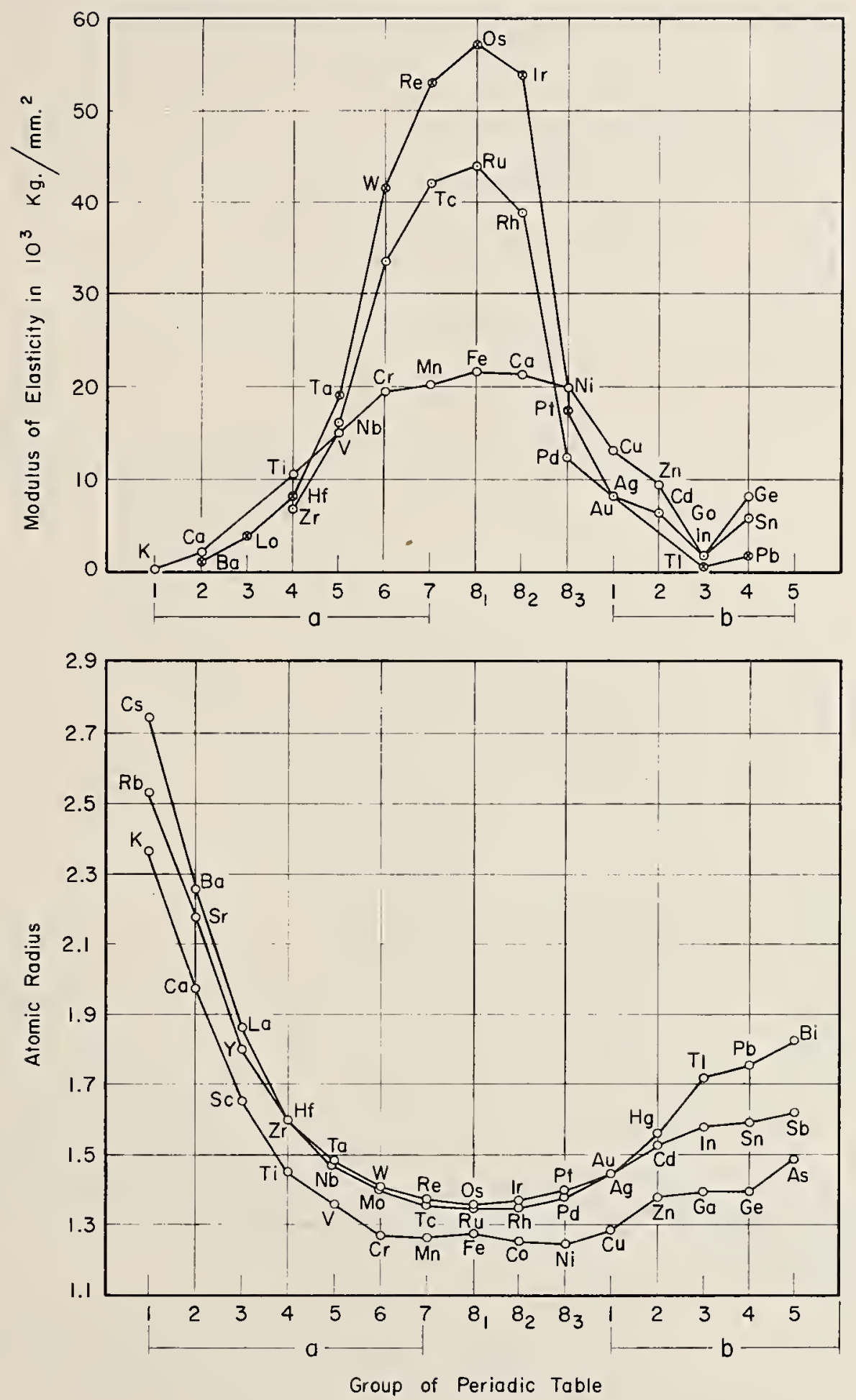

FIGURE 8. Periodic variation of the atomic radius and elastic modulus with atomic number for the first, second, and third long periods. 


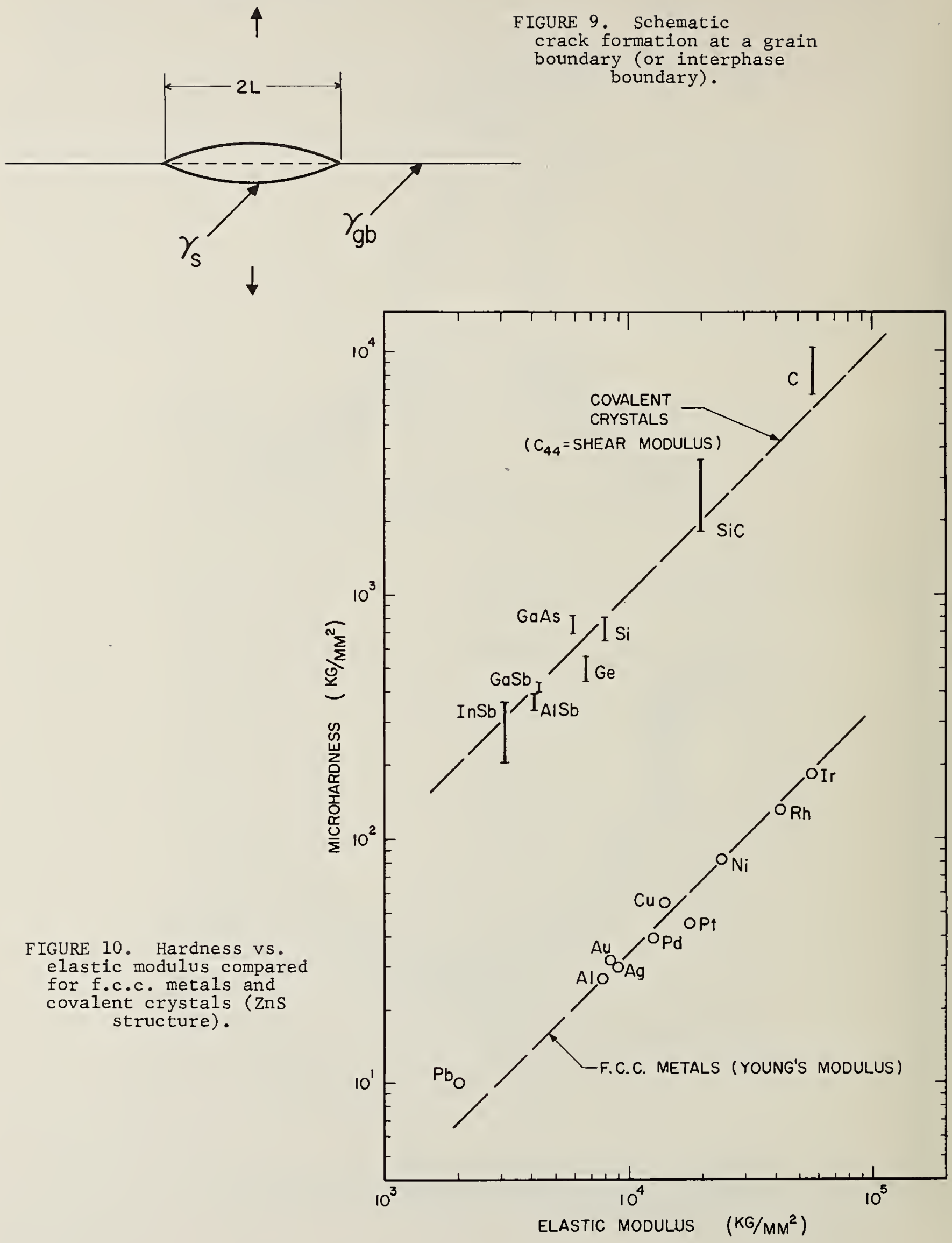




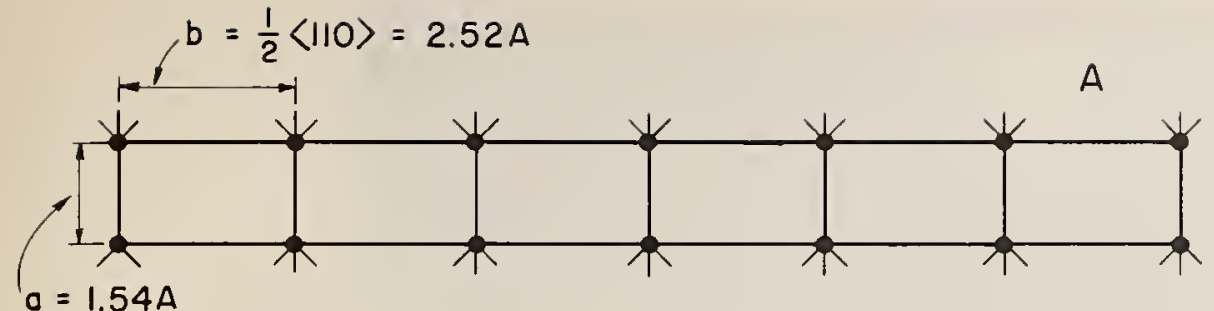

B

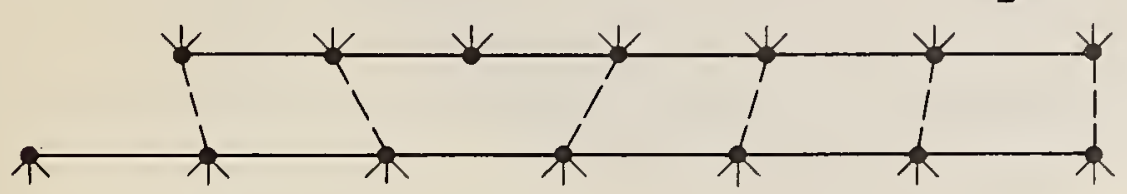

C

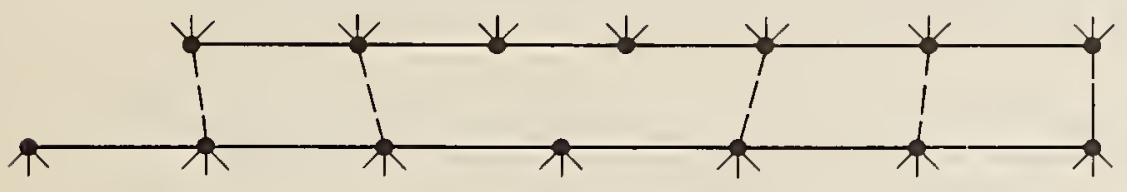

FIGURE 11. Schematic motion of the core of an edge dislocation in the diamond crystal structure. Each atom has four bonds in the lattice.

(A) Strip of atoms lying along the glide plane in a $<110>$ direction; \{112\} plane lies para1lel to paper. No dislocation present.

(B) Dislocation core in symmetric position.

(C) Dislocation core moved to second symmetric position.
FIGURE 12. Correlation between activation energies for dislocation motions and elastic stiffness.

(Data from Chaudhuri, Patel, and Rubin.)

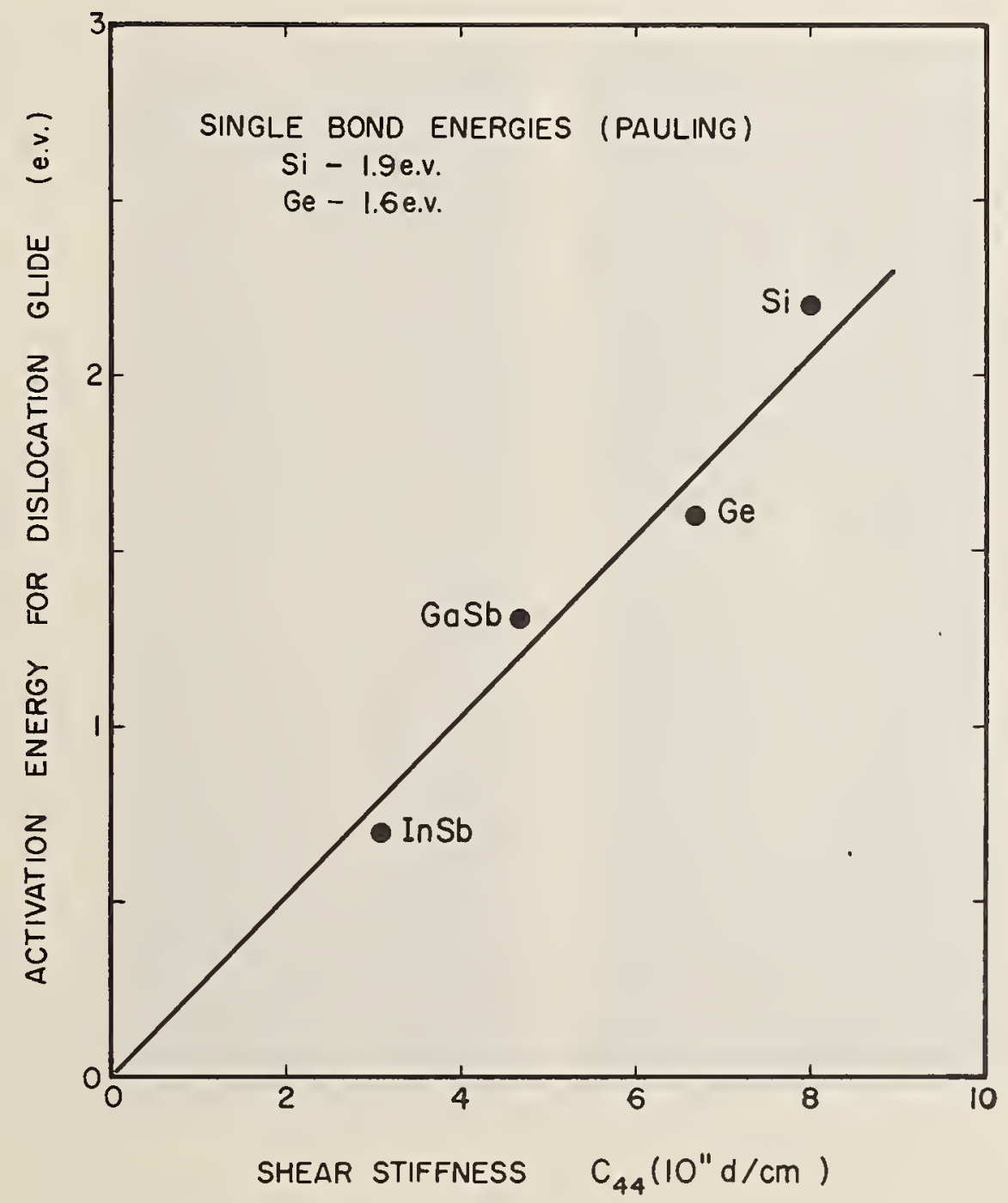




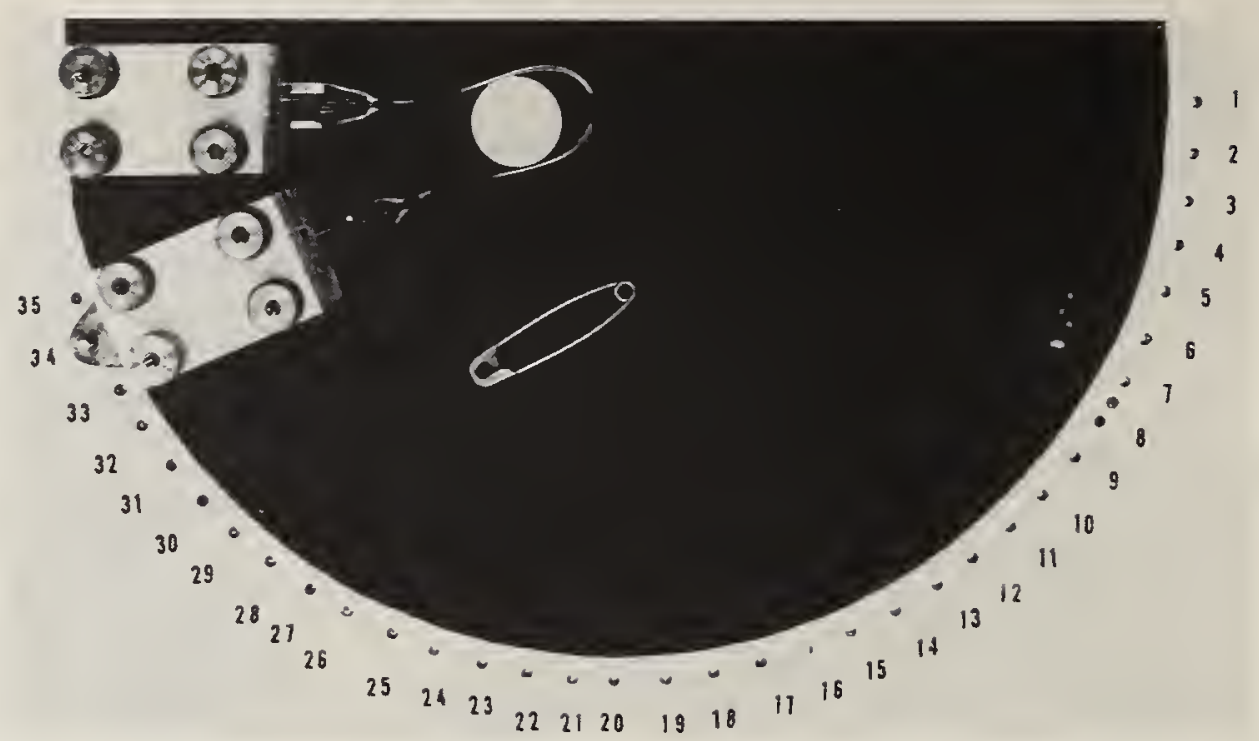

FIGURE 13. Drawn fused silica rod of large diameter bent to elastic strain of about 10 percent.

(courtesy of W. B. Hillig.)

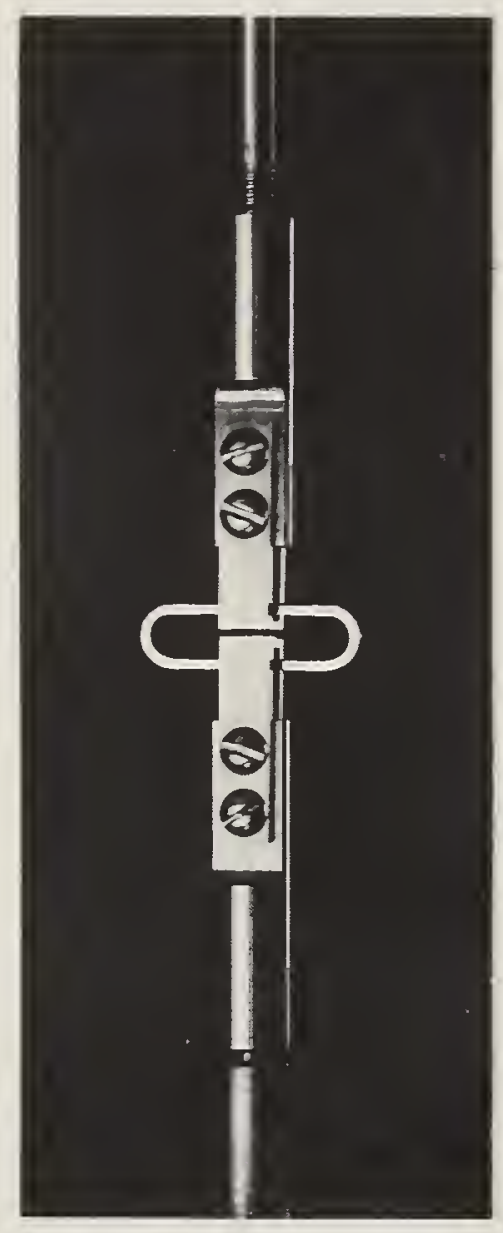

(a)

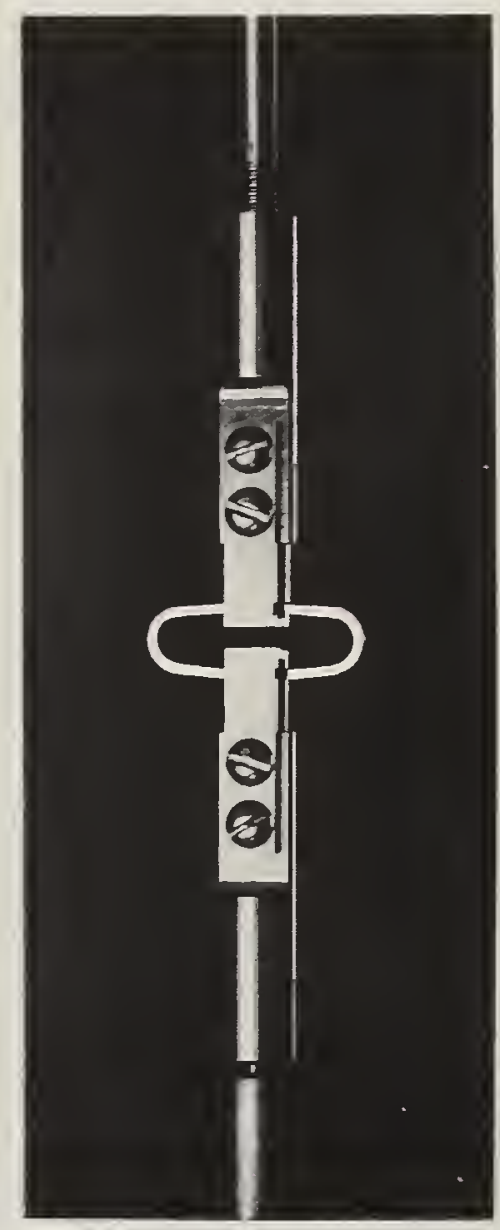

(b)
FIGURE 14. Ring made from a silicon crystal and chemically polished. The ring is about 1.5 in. long and $1 / 16$ in. square in cross section.

(a) No load.

(b) Just prior to fracture - 500,000 psi maximum tensile stress. 
A REVIEW OF THE EFFECT OF MICROSTRUCTURE ON

MECHANICAL BEHAVIOR OF POLYCRYSTALLINE CERAMICS

$$
\text { W. D. Kingery and R. L. Coble }
$$

Ceramics Division, Department of Metallurgy Massachusetts Institute of Technology

Cambridge, Massachusetts

In polycrystalline ceramics, grain boundaries and porosity occur and their effects must be explicitly evaluated to properly interpret experimental property measurements. The effects of these variables on ductility, brittle fracture, and creep fracture processes are substantial and must be considered in addition to observations on single crystal behavior. In addition to fracture phenomena, the rate of deformation of polycrystalline materials is generally different from that observed for single crystals of the same composition. The resultant properties of these materials thus are more complex than single crystals; the effect of the added variables is important for al1 mechanical behavior and controls the observed behavior for many systems.

The present review is not exhaustive, but rather considers typical results on which is based current understanding of effects of porosity and grain size on strength, elasticity, creep, and creep rupture. Effects of porosity on elasticity and of grain size on strength and creep rate are now understood; effects of porosity on strength and creep rate and of grain size on fracture are not we11 understood, but useful empirical correlations are available.

\section{Introduction}

Any understanding of the mechanical behavior of complex ceramics must be based on investigations of the simplest systems available. Variables of stress, strain, strain rate, composition, temperature, and environment are in themselves sufficiently complex that additional microstructural variables make understanding extremely difficult. The earliest characterization of ceramic mechanical properties was developed for single crystals and homogeneous glasses in which microstructure and orientation variations were absent; even now it cannot be said that we completely understand the deformation behavior of these relatively simple systems in a quantitative way. Most of the discussion in the present Symposium has thus properly dealt with the behavior of we11defined single crystals leading to an understanding of the deformation and fracture processes that occur in them.

In some materials quantitative understanding of single-crystal deformation and fracture phenomena must be developed before microstructural variables can be understood, because the presence of pores or grain boundaries or other phases simply modifies the single crystal behavior. For example, flow and fracture in Mgo is modified by the presence of grain boundaries, but the same mechanism for flow and cleavage criteria for fracture apply to both single and polycrystalline specimens. In other materials the deformation or fracture mechanism may be completely altered by the microstructure. Polycrystalline alumina deforms by a diffusional process because the basal slip process which operates in single crystals is inhibited by the presence of grain boundaries. In cases such as this, direct study of microstructural variables is essential, and understanding of the single crystal behavior only delineates the regions of temperature and stress where different processes are rate controlling.

Real ceramic systems are complex in composition and structure with one or more crystalline phases and porosity normally present in variable form and distribution. A complete quantitative treatment of the effects of these variables on mechanical properties is beyond the scope of the present discussion, although this must be an aim of future ceramic research. Ultimately, we want to be able to predict and improve on the behavior of complex real ceramic systems by applying a thorough understanding of their limitations and capabilities.

In the present discussion we will extend our consideration beyond single crystals and homogeneous glasses to polycrystalline ceramics, which include as new variables the effects of grain boundaries, grain size, grain orientation, and porosity. These additional variables have a significant effect on almost al1 mechanical behavior and a controlling effect on many important characteristics. In order to summarize our present understanding, correlations between mechanical properties and physical models will be presented, and empirical relationships will be shown where no physical models now available appear applicable. 


\section{Fracture}

A discussion of fracture requires separate consideration of the different possible modes of fracture in order to evaluate properly the effects of specific variables. The fracture mechanism usually changes with temperature, and we will discuss effects of microstructure on brittle fracture, which is commonly observed for ceramic materials at low temperatures, and creep fracture, which is observed at high temperatures.

\subsection{Brittle Fracture}

Brittle fracture, that is, low-energy fracture accompanied by little or no plastic deformation, is commonly observed for polycrystalline oxides and other more complex ceramic materials. As has been discussed previously $[1]^{1}$, the minimum requirement for crack propagation is that the strain energy released be equal to the new surface energy formed. Because observed strengths of typical ceramics are less than $E / 100$, various imperfections are as sumed to be present, with crack propagation the important fracture criterion. In readily deformable ceramics (MgO, NaCl) crack nucleation resulting from plastic deformation is an important part of the overall fracture process.

\section{a. Effect of Porosity}

Porosity is a phase almost always present in polycrystalline ceramics and decreases in strength with increasing porosity have been observed in many single phase and polyphase metal and ceramic materials. In general, the effect of porosity has not been totally separated from the combined effect of porosity and grain size. Samples prepared by sintering or by hot pressing powder compacts general1y exhibit an optimum strength under particular fabrication conditions. Porosity is the principal variable at low temperature and short processing times and grain size is the principal variable at high temperatures and long times. Samples with small initial particle size generally exhibit higher strengths at their optimum preparation treatment than samples prepared with larger particle size $[2,3]$. At the present time, changes in strength with porosity have only been treated empirically, although various structural factors such as pore shape and distribution which cause changes in the average stress level within the matrix in terms of the applied stress, and stress concentration factors associated with pores and surface features are recognized. These have not, however, been assembled into a unified model by which observed strength changes with porosity can be predicted.

One of the difficulties in evaluating strength data results from the fact that the pore shape changes during the course of sintering and it is by sintering that most samples have been prepared. Stress concentration factors change from high values $(\sim 1000)$ for flat elliptical cracks to low values ( 2 to 3 ) for cylindrical or spherical cavities. Though the pore shape changes in a definite way during firing, the exact shapes or distribution of shapes have not been sufficiently well studied, nor have the associated stress concentration factors for those shapes been elucidated. Both are necessary to combine the stress concentration factors with average stress calculations for predicting changes in strength with porosity.

Perhaps the best way of illustrating the problem of analyzing the effects of porosity on strength is to show the variability of elastic modulus changes with porosity. The influence of changing porosity on the elastic modulus has been analyzed by Mackenzie for spherical pores [4]. For Poisson's ratio equal 0.3 and semi-empirical evaluation of the constant for the quadratic term, he found that the ratio $\mathrm{E} / \mathrm{E}_{\mathrm{P}=0}=1-1.91 \mathrm{P}+$ $0.91 \mathrm{P}^{2}$ where $\mathrm{P}$ is the volume fraction porosity. Good agreement between experimenta1 and predicted changes in elastic modulus with changing porosity was reported by coble and Kingery in specially prepared samples having a continuous solid phase containing nearly spherical pores [5]. Other measurements of changes in elastic modulus with porosity have used samples prepared by normal sintering or hot pressing [7]. As a consequence, the pores are not a11 of equivalent shape and produce different stress concentration effects. Empirical treatment of data for alumina has been done by ${ }_{\mathrm{P}} \mathrm{Knud}$ sen $[8]$ and Spriggs [ $\dot{9}]$. They find that the data can be expressed as $E / E_{p=0}=e^{-b P} P_{\text {Where }}$ the empirical constant $\dot{b}$ determined from different investigators data varies between 2.37 and 4.35. The lowest value, 2.37 (excluding Lang's data, whose samples only covered the range from 0.11 to $2.78 \%$ porosity), corresponds to the elastic modulus changes for a discontinuous pore phase in a continuous solid matrix; the higher values presumably result from changes in pore shape. Knudsen [8] grouped all the data together

${ }^{1}$ Figures in brackets indicate the literature references on page 110. 
and calculated an average single empirical constant of 3.95 .

Assuming that the average stress in the matrix changes inversely with the elastic modulus, the strength might also be assumed to be directly proportional to the change in elastic modulus times a stress concentration factor associated with the pores. However, strength data follow an exponential function, $\sigma / \sigma_{\mathrm{P}=0}=\exp (-\mathrm{bP})$ with an empiricaI coefficient, b, about twice that observed for elastic modulus changes in equivalent bodies. Thus, for Coble and Kingery's results [5], $\mathrm{E} / \mathrm{E}_{0}=\exp (-2.5 \mathrm{P}$ ), (for the

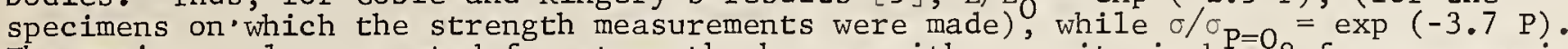
The maximum value reported for strength changes with porosity is $\mathrm{b}^{\mathrm{P}}=0$ for compression strength $[10,11]$. These relationships are illustrated in figure 1 . There is only a small difference between the theoretical elasticity relationship of Mackenzie for isolated spherical pores and the values most commonly observed in sintered or hot pressed bodies. However, strength data show a much stronger dependence in porosity. Expected changes in pore shape as a function of firing time and temperature would increase the stress concentration factors with increasing porosity. Without specific knowledge of the shape change, the change in stress concentration factors, and the strength changes as functions of porosity cannot be predicted. In some cases, such as stainless steel formed under pressure giving flat elliptical pores and materials like graphite, titania, and aluminum titanate which form flat grain boundary cracks as a result of anisometric contraction during cooling, a small fractional volume of porosity can have a disastrous effect on strength.

We may summarize the effects of porosity on strength by noting that these are qualitatively understood. They are generally larger than the effects predicted for single shapes of pores. So far as we are aware there has not been a quantitative investigation of the effect of pore morphology on the fracture process.

The large effect of porosity on mechanical properties leads to difficulties in the interpretation of other variables affecting fracture processes in most ceramic systems. Because of changes in pore morphology with different firing treatments, an uncontrolled variable of substantial importance is normally included. This has frequently not been explicitly considered in interpretation of the-mechanical properties of polycrystalline ceramics.

\section{b. Effect of Grain Size}

Decreases in strength with increasing grain size have been observed in aluminum oxide $[3,7,12]$, beryllium oxide [13], thorium oxide [8], uranium oxide [14], magnesium oxide $[7]$, and chromium carbide [8]. Decreases in strength as a function of precipitate crystal size in crystal-glass composites have also been observed in silicate porcelains and in Pyrocerams. Most of the data is available on samples that have been sintered or hot pressed such that there is a simultaneous change in porosity and grain $-8 i_{\mathrm{P}} \mathrm{e}_{\mathrm{g}} \mathrm{n}$ For these, empirical formuli have been developed of the form $[8] \sigma / \sigma \mathrm{p}=\mathrm{Q}_{\mathrm{f}} \mathrm{k} \mathrm{e}^{-\mathrm{BP}} \mathrm{G}$
With simultaneous changes in porosity and grain size, calculations of from the above equation leads to values of $b \cong 8$ and $n=1 / 2$ or $n=1 / 3$.

At high density the change in grain size is the most important variable. For example, above about 95 percent porosity, the strengths of alumina samples exhibit values ranging from 70-80,000 psi at $1 \mu$ grain size down to 20,000 psi at about $100 \mu[7]$. In this density range, the exponent for the grain size dependence of strength only changes from $n=1 / 4$ to $n=1 / 3$ when the porosity correction is introduced as shown in figure 2. The exponent $n=1 / 3$ is not understood on a theoretical basis. It has been reported for hot pressed aluminum oxide [7], and magnesium oxide [7] and for sintered aluminum oxide [27; Knudsen has found that $\mathrm{n}=0.4$ and 0.55 for thoria and chromium carbide respectively $[17]$. For one group of beryllium oxide specimens [13] it was reported that $n=1$.

A value of $n=1 / 2$ is predicted from a model based on fracture propagation following cleavage where the initial cleavage crack is equal to the grain size. The $n=1$ value observed in beryllia is understandable on the basis of internal stresses on the grain boundaries which result from anisotropy in thermal expansion. However, with the later report that the grain size dependence of the strength in other sintered beryllia specimens gave $n=1 / 3$, the applicability of the boundary stress model is not easily decided. The $n=1 / 3$ value is not predicted at the present time by any simple model. For magnesium oxide it is possible that this dependence is based on dislocation pileup which produces cleavage either in the grain beyond the boundary or at the grain boundary itself [15]. Whether or not this is an appropriate functional dependence must await further clarification of the stresses involved in dislocation pile-ups as a function of grain size. 
Extrapolation of the strength as a function of grain size to one micron for aluminum oxide specimens gives 60,000to 110,000 psi. Measurements of the strength of $60^{\circ}$ sapphire single crystals abraded and annealed at room temperature and at liquid nitrogen temperature give strengths which vary from 40,000 psi to 100,000 psi [17]. Wachtman has observed aluminum oxide single crystal strengths up to 140,000 psi [18]. The equivalence of the strength of one micron polycrystalline samples with single crystal data suggests that the fracture origin is not significantly influenced by internal stresses in micron-size polycrystalline materials. Gilman [19] has analyzed the situation for brittle materials and concluded no grain size effect occurs without a second phase at boundaries.

\section{c. Boundary Processes}

There are several ways in which grain boundaries may be considered to contribute to the fracture process. The simplest is the influence that the boundaries have on the surface topography of an equilibrium fired body. The interaction of the surface energy with the grain boundary energy results in grooves etched on the surface that cause stress concentrations. Equilibrium grooves should be geometrically equivalent regardless of grain size. It is therefore difficult to understand how these grooves would introduce a grain size dependence of strength. A second effect which the boundaries can introduce is to provide obstacles for gliding. The influence of boundaries on inhibition of dislocation movement has been observed as a direct so urce of cracks leading to failure. Johnston et al. [20] have observed in magnesia bicrystals that the crack which formed at a dislocation pileup at the boundary changed from being intergranular to a cleavage crack in the grain ahead of the dislocation pileup, depending upon the orientation difference between the crystals. They also observe that the stress level at which cracking first occurred was essentially equal to the yield stress and that fracture would ensue at a stress not much higher than the yield stress. For many magnesia specinens on which strength data have been reported, strength values observed were 20,000 psi in agreement with the observed yield strength. On the other hand, for small grain size specimens of hot pressed magnesia, the strengths observed by Spriggs and Vasilos [7] were 40,000 psi. This higher strength may result from the fact that the length of the pileup required to produce a sufficient stress concentration to cause subsequent failure in the bicrystal experiments exceeds the grain size and the pileups essentially cannot or do not form in small grain sized specimens unless a higher stress is present.

The influence of the boundary on stopping a cleavage crack from penetrating the next grain provided the basis for a model by which Orowan [21] rationalized the effect of grain size on the strengths of steels at low temperatures. That model leads to a predicted grain size dependence on strength of $\mathrm{n}=1 / 2$. Finally, a model for the influence of boundaries on fracture, based on internal stresses resulting from thermal expansion anisotropy present in many ceramic materials, has been developed [23]. Shear stresses are present at the boundaries and the change in shear stress as a function of grain size leads to a prediction that strength should change as the grain size with $\mathrm{n}=1$.

From the variable behavior observed experimentally, it is clear that fracture processes for a range of materials are more varied than was assumed earlier. None of the simple models based on crack initiation due to internal stresses, or fracture propagation based on existing cleavage cracks equivalent to the grain size, or of fracture initiation by dislocation pileups explain all the existing data.

In cases where fracture is preceded by plastic deformation within individual grains and with dislocation pileups and stress concentrations at grain boundaries, the resultant stress concentration will increase as the grain size increases, such that finer grain samples will have higher fracture strength than large grain size samples. In an analysis of this process, J. J. Gilman [23] estimated an optimum grain size of about one micron for typical materials. Polycrystalline oxide samples have not been investigated in this size range free from effects of variables such as porosity. 0bservations of stress-strain curves of bicrystals of lithium fluoride by Feuerstein [24] indicate that grain boundaries in ionic crystals are not inherently weak. He found that plastic deformation in at least one grain always preceded fracture, but that the presence of grain boundaries was a strong barrier to dislocation motion, leading to stress concentrations. As a result, failures took place in a brittle fashion.

\subsection{Delayed Fracture (Static Fatigue)}

Single crystal aluminum oxide exhibits static fatigue; that is, the strength decreases as a function of stress duration or with decreased loading rates [25]. The effect is attributed to corrosion by water vapor. High strengths are observed in low 
temperature or vacuum testing, and the fatigue coefficient is essentially eliminated in comparison with room or higher temperature measurements. Polycrystalline samples similarly exhibit fatigue [17]. It is significant that there is essentially no difference in the fatigue behavior between polycrystalline and single crystalline samples. Thus the presence of the grain boundaries does not provide a site which, because of its higher energy, is preferentially attacked and does not preferentially weaken the alumina matrix.

\subsection{Ductile Fracture}

In single crystals of alkali halides and magnesium oxide at room temperature substantial ductility is observed prior to fracture, and crack initiation results from the interaction of slip bands. The tensile strain of favorably oriented crystals reaches above 20 percent before failure occurs. In some cases, brittle fractures result from surface imperfections.

In contrast, polycrystalline materials are observed to show little ductility prior to brittle fracture. This results from the fact that grain boundaries act as barriers to deformation, giving rise to stress concentrations and crack initiation after small amounts of deformation. The difficulty in transmission of slip across grain boundaries suggests that while ductile polycrystalline oxide ceramics are not likely to be achieved at room temperature, a decrease in grain size so that stress concentrations from slip are smaller can lead to higher strengths as already found for Mgo [7]. Thus, higher strengths can be achieved for some polycrystalline ceramics than is found for single crystals, even though little deformation occurs and the fracture is "brittle."

\subsection{Creep Fracture}

The strength of polycrystalline ceramics consistently decreases at approximately half the melting point. The most frequent interpretation of the change in strength with temperature is that fracture changes from transcrystalline at low temperatures to intercrystalline at temperatures where creep occurs. Considerable evidence has been accumulated to support this model from the creep and fracture behavior of metals. There are few studies of the fracture of ceramic specimens at high temperatures, and it is not known whether or not the fracture process generally changes from a transgranular to an intergranular one. For high density polycrystalline alumina specimens, Folweiler [26] has observed that the strength is independent of the strain rate at constant grain size and temperature. For high density alumina the strength is approximately 25,000 psi for small grain size material at $1400{ }^{\circ} \mathrm{C}$ and decreases to approximately 15,000 psi for large grain size material at $1700{ }^{\circ} \mathrm{C}$. These strengths are approximately one-third of the values measured for equivalent grain size specimens at room temperature. Metallographic examinations of specimens tested in bending revealed that pores and cracks were nucleated in a way depending on the stress level. At the neutral axis of the specimens, no pores or cracks were visible even after extensive strain. In tensile regions near the neutra1 axis isolated pores were observed to form on grain boundaries and at three grain corners. In regions further from the neutral axis, whole planar faces of boundaries perpendicular to the tensile stress separated from the adjacent grain. These cracks between grains subsequently became connected, forming continuous cracks. In the regions of maximum tensile stress cracks were present in profusion. This is unlike the room temperature behavior where essentially a single crack causes complete failure of the specimen.

Intrater and Machlin [27] conducted measurements on grain boundary sliding processes between metal bicrystals. They observed that the deformation was not entirely loca1ized to the boundary. They found that jogs would occur in the plane of sliding leading to smal1 gaps between the ends of the slide segments. It was their interpretation that the non-coplanar sliding process provided the source of pores and cracks frequently observed in creep measurements in metals.

In stress-rupture experiments with polycrystalline hydrogen oxide at temperatures near its melting point, behavior was observed similar to that found for many metals as shown in figure 3. There seems to be no indication that the correlation found for metals between creep strength and grain size is not also applicable to ceramics.

\section{Deformation}

Deformation in ceramic systems can occur by dislocation movement, by diffusional processes, and by localized grain boundary deformation. Each of these processes is important for polycrystalline ceramics under some circumstances. 


\subsection{Dislocation Processes}

Dislocation configurations and their movement through crystals to give deformation on certain slip systems have been discussed in detail in previous papers in this Symposium. Deformation rates depend on the stress, temperature, crystal structure, sample prior history, and the presence of impurities. In general, because of the directed bond nature of covalent structures and the electrostatic requirements in ionic materials, there are greater restrictions on dislocation configurations and movement in ceramic materials than are present in metals. Dislocations tend to have complex structure with large Burgers' vectors, are often split into partials separated by stacking faults, and operate only on a relatively few crystallographic planes. For ionic materials when one ion is highly polarizable, as is the case for silver chloride and mercury chloride, there is less resistance to dislocation movement, giving slip on more crystallographic planes than in highly ionic structures such as lithium fluoride and magnesium oxide.

It has been shown by Taylor [29] that the plastic deformation of a polycrystal aggregate by slip is subject to the restriction that each grain must remain in contact with adjoining grains at the boundary, and in the absence of grain boundary slip or twinning requires five independent modes of slip to occur. This result is particularly important for ceramic systems where the number of slip systems with easy slip is restricted. In hexagonal structures such as $\mathrm{Al}_{2} \mathrm{O}_{3}$ and $\mathrm{BeO}$, basal slip is the only mode commonly observed except at very high temperatures. In sodium chloride structure systems, such as magnesium oxide, $\{110\}$ slip is the only system commonly observed at room temperature. Thus, the operation of plastic flow mechanisms leads to stress concentrations at boundaries and cleavage fracture at small amounts of plastic deformation in polycrystalline ceramics at room temperature.

As a result, even for materials showing high ductility in single crystal form, we cannot anticipate the formation of ductile polycrystal bodies even when impurities are eliminated and grain size is controlled.

Inasmuch as secondary slip systems are often highly temperature dependent, the possibility of deformation of polycrystalline materials by plastic flow increases greatly when the temperature is raised. In sodium chloride and lithium fluoride single-crystals, slip only occurs on (110) planes at room temperatures. When deformation is carried out at temperatures above about $300^{\circ} \mathrm{C}$, however, concurrent slip occurs on (100) planes [30]. At temperatures above about $300^{\circ} \mathrm{C},(100)$ slip has al so been observed in polycrystalline samples of lithium fluoride which then becomes ductile [31]. Similarly, in magnesium oxide [32], evidence of (100) slip has been observed at temperatures above $400{ }^{\circ} \mathrm{C}$ with a critical resolved shear stress which decreases rapidly with temperature until at temperatures about $1000^{\circ} \mathrm{C}$ plastic deformation has been observed with (100) slip occurring in polycrystalline samples.

At high temperatures, even though dislocation motion is severely reduced through pileup on slip planes, steady-state deformation occurs by means of dislocation climb such as occurs during polygonization [33]. For dislocation climb weertman [34] has proposed that the creep rate should be proportional to the resolved shear stress raised to the $9 / 2$ power; in general, creep data for metals at high temperature show a strainrate stress dependence having some exponent greater than one and near four. Chang's data for single crystal sapphire and ruby show this general stress dependence [35 7 .

Creep data [36] for polycrystalline magnesia containing 2 to 5 percent porosity showed a stress dependence in the range $\varepsilon \sim \sigma^{2} \cdot 5-3 \cdot 5$. For large grain-size samples of aluminum oxide Warshaw [37] observed $\dot{\varepsilon} \sim \sigma^{4}$ at temperatures greater than $1800^{\circ} \mathrm{C}$. His observed creep rates were also significantly larger than those given by extrapolation of the diffusional creep process discussed in the next section. Thus, it seems clear that plastic deformation by dislocation processes occurs at high temperatures for magnesium oxide. Deformation of polycrystalline $\mathrm{Al}_{2} \mathrm{O}_{3}$ at high temperatures is controlled by dislocation processes together with either co-operative grain boundary deformation or slip on prismatic or pyramidal planes giving multiple slip systems.

\subsection{Diffusion Creep Processes}

An additional deformation process that can occur in polycrystalline materials is diffusional or Nabarro-Herring creep where self-diffusion within the grains of a polycrystalline solid permits the solid to yield under an applied stress. Deformation results from diffusional flow of atoms within each crystal grain away from those bound-aries where there is a normal compressive stress (high chemical potential) toward boundaries having a normal tensile stress. According to Herring $\lceil 38]$, this leads to a viscous flow (strain rate directly proportional to stress) of polycrystalline samples with an effective viscosity given by 


$$
\eta=\frac{k r^{2}}{4 D a}=\left(\frac{3}{32 \pi}\right)^{2 / 3} v_{g}^{2 / 3} \frac{k T}{D a}
$$

where $\mathrm{k}$ is the Boltzman constant, $\mathrm{T}$ the absolute temperature, $\mathrm{D}$ the diffusion coefficient, a the atomic volume, $I$ the radius of a spherical grain, and $v_{g}$ the grain vol-
ume.

Creep data at high temperatures for polycrystalline $\mathrm{Al}_{2} \mathrm{O}_{3}[37,35,40,41]$ and $\mathrm{BeO}$ [35] support the diffusional creep model over wide ranges of stress $\left(10^{2}-10^{4} \mathrm{psi}\right)$ and temperature $\left(1200\right.$ to $\left.1800^{\circ} \mathrm{C}\right)$. The creep rates in high-density polycrystalline alumina at equivalent temperatures and stresses are one to several orders of magnitude lower than those observed for single crystals. In addition, the character of the stress dependence at a fixed temperature and grain size is different from that in single crystals. In the polycrystalline samples the creep rate varies directly with the stress and the strain rate at constant stress is inversely proportional to the grain size squared in accordance with the diffusional model. While the stress and grain size dependence and magnitude of the creep rate are in reasonable agreement between different investigators, the absolute rates of creep deformation have varied by an order of magnitude.

Chang [35] found good agreement between the creep deformation rate of beryllium oxide and measured diffusion coefficients. Recently, Paladino and Coble [42] have found reasonable agreement between the diffusional creep rate of polycrystalline aluminum oxide and directly measured diffusion coefficients in this system. It thus seems clear that the microstructure of polycrystalline ceramics at elevated temperatures must be directly considered in determining deformation characteristics, and at high temperatures the deformation behavior of fine grain-size polycrystalline oxides is directly related to the grain size. This is particularly the case for aluminum oxide where the measurements of Warshaw indicate that different deformation mechanisms occur for the fine grain and large grain samples leading to differences in temperature dependence, grain-size dependence, and stress dependence of the deformation rate. The inclusion of microstructure as a variable is essential for proper interpolation and extrapolation of available experimental data.

\subsection{Grain Boundary sliding}

A third process by which deformation can occur in polycrystalline ceramics is grain boundary sliding. High-angle grain boundaries are areas of poor lattice fit or high dislocation concentration which give rise to complex deformation processes intimately associated with the presence of a grain boundary. It was shown by $\mathrm{Ke}$ [43] that grain boundaries behave viscously with the strain rate proportional to the stress when a shearing stress is applied, but that the deformation process in grain boundaries is strongly structure sensitive depending on cold work and impurity additions [44]. The detailed mechanism of grain-boundary deformation is not clear at the present time, and indeed the detailed structure of grain-boundary configurations in ceramic systems is not clearly understood. By itself, without auxiliary deformation processes, grain-boundary sliding cannot contribute to deformation of a continuing nature since interior deformation or diffusion processes must occur coincidentally to fulfill geometric continuity requirements. Thus grain-boundary sliding can have an indirect effect in cooperation with diffusional creep mechanisms or plastic flow processes to increase the deformation rate and reduce the geometric restrictions on other deformation processes. Probably, its most important effect is the added degree of freedom with regard to interior slip processes in grains. Mullendore and Grant [45] have recently concluded from studies of tensile creep in metals that grain-boundary sliding is a consequence of interior slip, since it provides an additional degree of freedom for slip crossing at grain boundaries. slip in one grain proceeds to the next by slip on one system plus boundary sliding.

\subsection{Influence of Porosity on High Temperature Deformation}

The influence of porosity on deformation has not been systematically studied in many materials over a wide range of porosity. Coble and Kingery [5] have reported on the influence of 4 to 50 volume percent porosity in polycrystaliine alumina. These measurements were conducted in torsional creep, and it was found that the maximum shear stress at a constant creep rate decreased by a factor of 50 as porosity increased from 5 to 50 percent (figure 4). Conversely, the creep rate at constant stress increased by a factor of 30 from 8 percent to 50 percent pores. In their specimens the pore size was large in comparison to the grain size and this large effect may result from offsetting of individual grains into the pores in order to relieve the stress. Since the equivalent changes in elastic modulus were a factor of five for equivalent changes in porosity, it is seen that the influence on creep rates or on maximum shear stress at constant creep rate is larger by a factor of ten than the change in the average stress level within the matrix. 
There have been a few other observations of creep rates in slightly porous specimens such as Wygant's data for magnesium oxide [36]; he also observed that porosity had a markedly deleterious effect on the creep resistance. Other effects of porosity on creep rate can be inferred from comparing the works of individual investigators, but such a comparison is not warranted because of the general scatter in results. Chang for example, observed that the addition of chrome oxide to polycrystalline alumina siightly increased the creep rate; this might be interpreted as an effect of porosity because the chrome-containing samples.contained a slightly higher pore content than the nonchrome-containing samples.

The large influence of porosity in alumina can be interpreted on the basis of a diffusional creep mechanism with grain boundary sliding as an accessory process. In fully dense specimens the maximum contribution by grain boundary sliding is limited to the contribution resulting from diffusional creep. If pores are present at three grain corners, the amount of material which must be diffused away in order to relieve the stresses which accumulate by the boundary sliding process will be much reduced. Consequently, the contribution of grain boundary sliding can be significantly enhanced when pores are present.

\section{Summary}

From available experimental data, it is clear that microstructure features have a strong effect on the mechanical properties of polycrystalline oxides. In particular, the presence of porosity in almost all ceramics formed in the usual manner leads to substantial changes in both fracture and deformation as compared to completely dense samples. This effect of porosity obscures detailed evaluation of grain size in much of the experimental data that is available. There is a real need for better data on porefree polycrystalline samples in order to separate out the effects of porosity and grain size.

Grain boundaries influence both fracture and deformation properties. They lead to limitations on the ductility of ceramic oxides at room temperature, since dislocations are effectively halted at boundaries between crystals. In addition, stress concentrations resulting from anisometric expansion are effective in changing properties. Deformation of polycrystalline oxides is less rapid than single crystals at high temperatures, since deformation commonly occurs by boundary sliding and Nabarro-Herring diffusional creep rather than plastic slip processes. Data available for polycrystalline oxides indicate that microstructure characteristics are often more important in determining overall behavior than is the chemical composition or crystal structure.

This paper was prepared at the Massachusetts Institute of Technology as part of a research program sponsored by the U. S. Atomic Energy Commission under Contract No. $\mathrm{AT}(30-1)-1852$. Jerry $\mathrm{E}$. Turnbaugh assisted in collecting and correlating the data and critically reviewed the final manuscript.

References

[1] T. L. Johnston, "Mechanisms of Fracture," this Symposium.

[2] R. Charles and R. Shaw, G. E. Research Laboratory Final Report Task No. 9, Armour Research Foundation Project No. 8203 (1961).

[3] I. B. Cut1er, J. Am. Ceram. Soc. 40, 20 (1957).

[4] J。 K. Mackenzie, Proc. Phys. Soc. (London) 63B , 2 (1950).

[5] R. L. Coble and W. D. Kingery, J. Am. Ceram. Soc. 39, 377 (1956).

[6] S. M. Lang, NBS Mono. 6, 45 pp. (1960).

[7] R. M. Spriggs and T. Vasilos, Bu11. Am. Ceram。Soc. 40, 187 (1961).
[8] F. P. Knudsen, J. Am. Ceram. Soc. 45, 94 (1962).

[9] R. M. Spriggs, J. Am. Ceram. Soc. 44, 628 (1961).

[10] W. Duckworth, J. Am. Ceram. Soc. $\underline{36}$, 68 (1953).

[11] E. Ryschewitch, J. Am. Ceram. Soc. 36, 65 (1953).

[12] W. B. Cranda11, D. H. Chung, and T. J. Gray, The Mechanical Properties of U1tra Fine Hot-Pressed Alumina, State University of New York, College of Ceramics at Alfred University, February 29, 1960.

[13] W. H. Duckworth and A. Rudnick, in Symposium Design with Brittle Materia1s, MAB Report MAB-175-M, Washington, D. C. (196I). 
[14] F. P. Knudsen, H. S. Parker, and M. D. Burdick, J. Am. Ceram. Soc. 43 , 641 (1960).

[15] R. J. Stokes and T. L. Johnston, and C. H. Li, Phil Mag. 31, 718 (1958).

[16] M. L. Kronberg, Acta Met. $\underline{5}, 507$ (1957).

[17] F. P. Knudsen, J. Am. Ceram. Soc. 42 , 376 (1959).

[18] J. B. Wachtman, Jr., and L. H. Maxwel1, J. Am. Ceram. Soc. 37, (7), 291 (1954).

[19] J. J. Gilman, Acta Met. $\underline{8}, 665$ (1960).

[20] T. L. Johnston, personal communication.[36]

[21] E. Orowan, Reports on Prog. Phys. XII, p. 185 (1949).

[22] R. L. Coble, Ch. 22, Ceramic Fabrication Processes, W. D. Kingery, Ed., John Wiley \& Sons (New York,1958).

[23] J. J. Gilman, Acta Met. 8 , 665 (1960).

[24] S. Feuerstein, Ph.D. Thesis, University of California (1962).

[25] R. J. Charles, Fracture, p. 225-249, John Wiley \& Sons (New York,1959).

[26] R. Folweiler, private communication.

[27] J. Intrater and E. S. Machlin, Acta Met. I, 140 (1959).

[28] W. D. Kingery, unpublished data.

[29] G. I. Taylor, J. Inst. Metals, 62, 307 (1938).
[30] R. D. Carnahan, T. L. Johnston, R. J. Stokes, and C. Niki, Trans. Met. Soc. AIME, 221 (2) (1961).

[31] W. D. Scott and J. A. Pask, private communication.

[32] C. O. Hulse, S. M. Copley, and J. A. Pask, private communication.

[33] M. L. Kronberg, Science 122, 599 (1955).

[34] J. Weertman, J. App1. Phys. 28, 363 (1957).

[35] R. Chang, J. Nuclear Materials 1 174 (1959).

J. F. Wygant, J. Am. Ceram. Soc. 34, 374 (1951).

[37]

[38]

S. I. Warshaw, Sc.D. Thesis, M.I.T. (1961).

C. Herring, J. App1. Phys. 21, 423 (1950).

R. Chang, J. Nuclear Materials 1 , 174 (1959).

[40] R. C. Folweiler, J. App1. Phys. 32, 773 (1961).

[41] E. K. Beauchamp, G. S. Baker, and P. Gibbs, WADC Project No. 0(7-7350) (Sept. 1961).

[42] A. E. Paladino and R. L. Coble, personal communication.

[43] T. S. Ke, Phys. Rev. 71, 41, 533 (1951).

[44] T. S. Ke, J. App1. Phys. 21, 415 (1950).

[45] A. W. Mullendore and N. J. Grant, Trans. Metals AIME, in press (1962). 


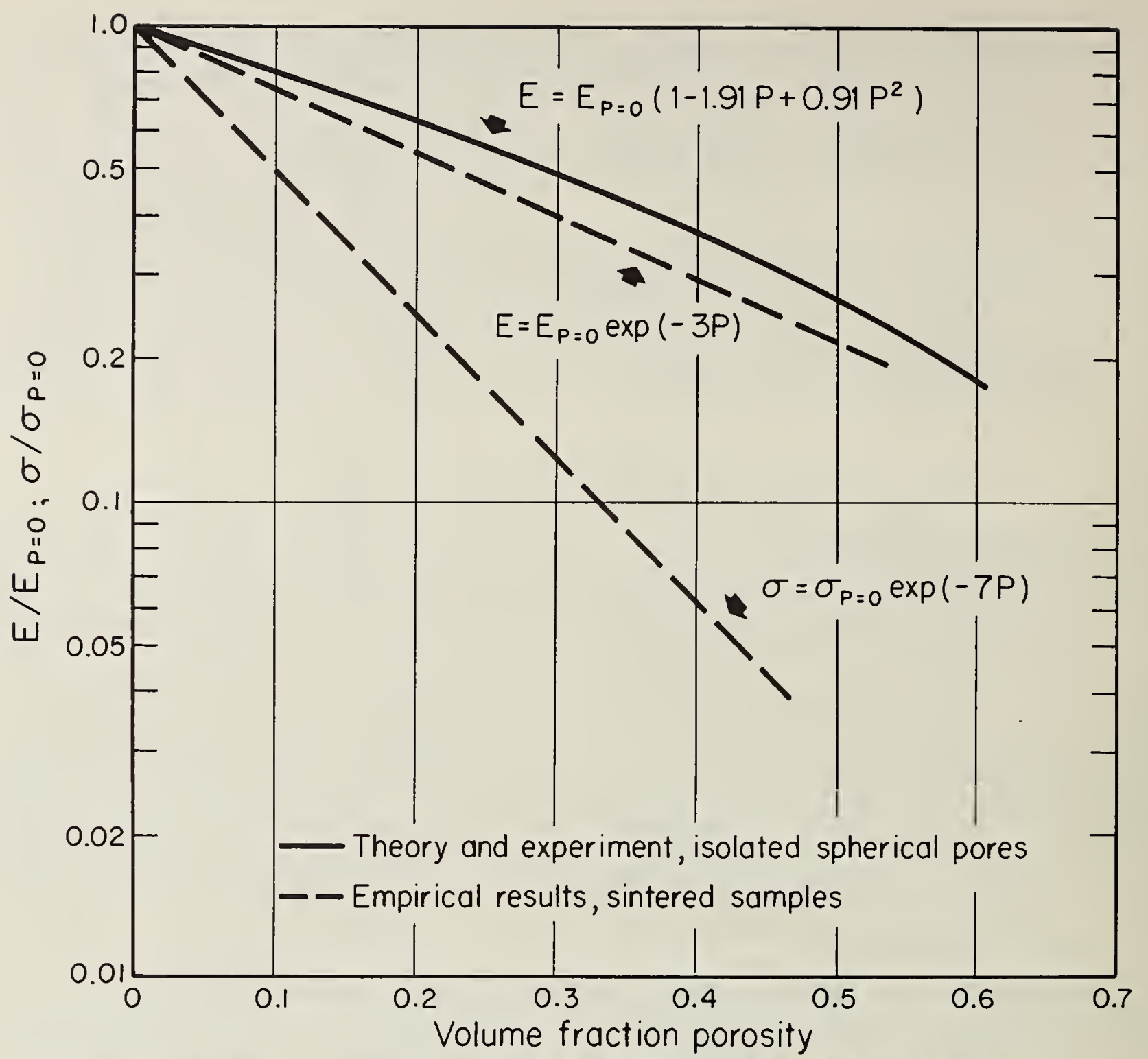

FIGURE 1. Effect of porosity on the elastic modulus and fracture strength of ceramics.

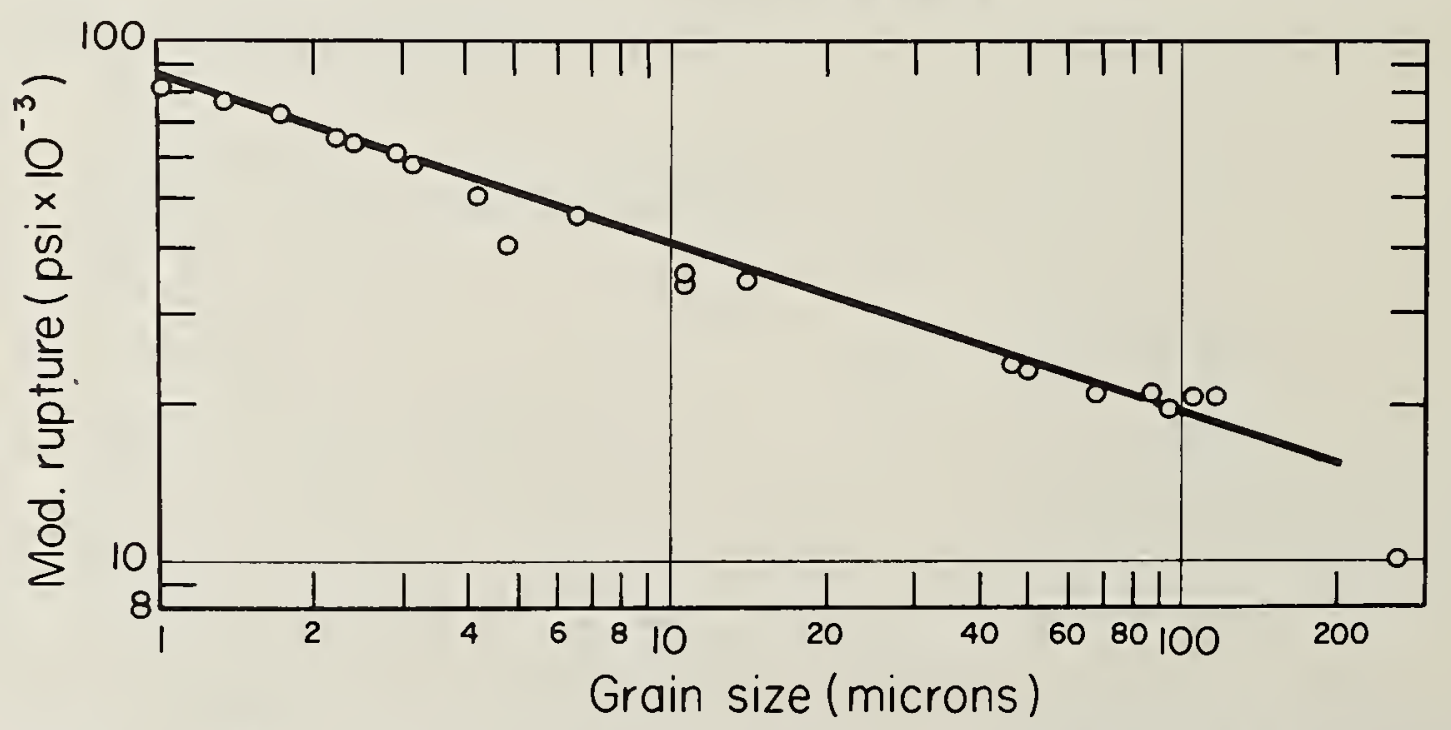

FIGURE 2. Effect of grain size on strength of polycrystaline aluminum oxide [7]. 

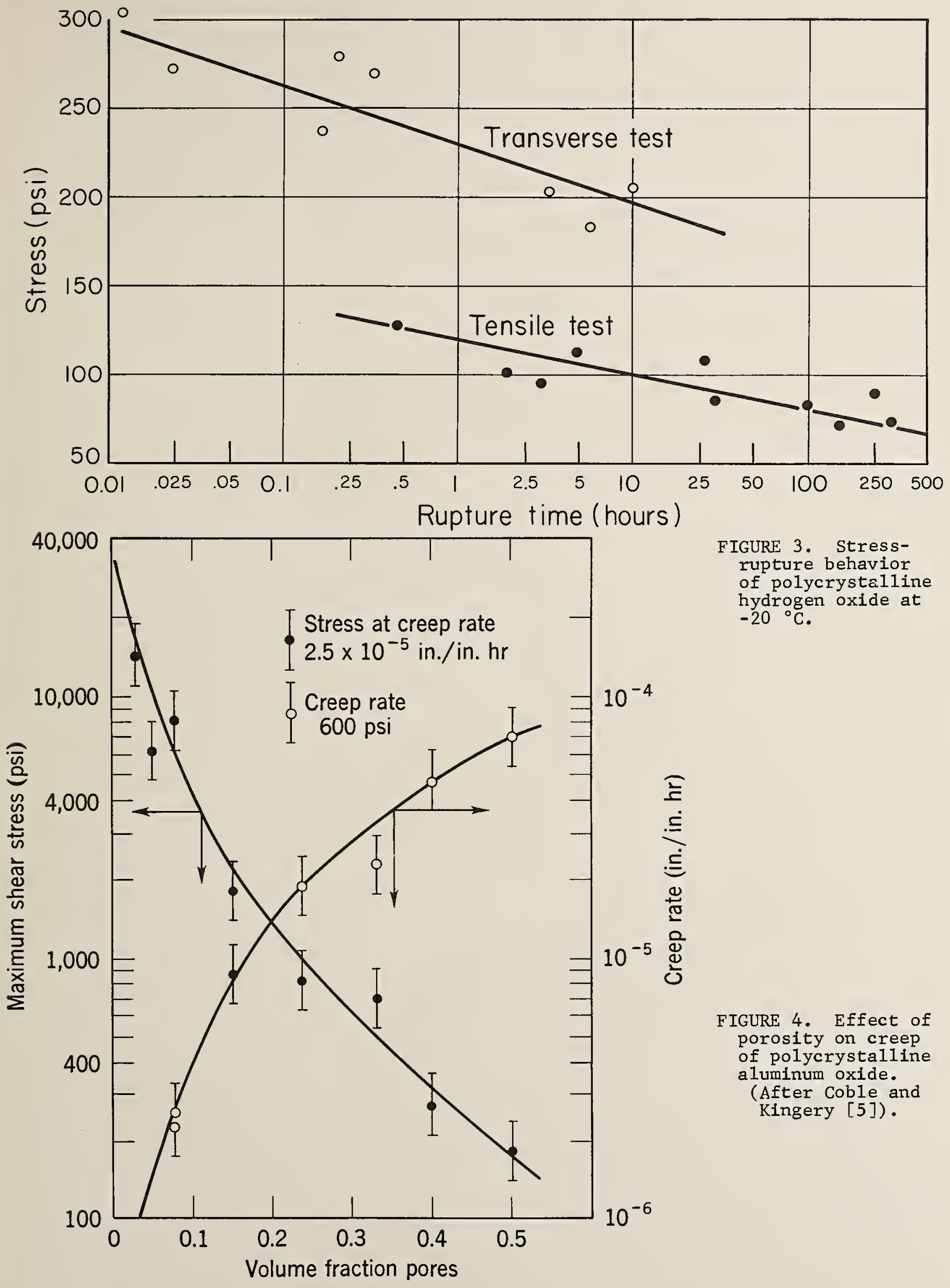

FIGURE 3. Stressrupture behavior of polycrystalline hydrogen oxide at $-20{ }^{\circ} \mathrm{C}$.

FIGURE 4. Effect of porosity on creep of polycrystalline aluminum oxide. (After Coble and Kingery [5]). 







\section{.}


